\title{
Patterns of synchrony in complex networks of adaptively coupled oscillators
}

\author{
vorgelegt von \\ M. Sc. \\ Rico Berner \\ ORCID: 0000-0003-4821-3366 \\ an der Fakultät II - Mathematik und Naturwissenschaften \\ der Technischen Universität Berlin \\ zur Erlangung des akademischen Grades \\ Doktor der Naturwissenschaften \\ - Dr. rer. nat. - \\ genehmigte Dissertation \\ Gutachter: Prof. Dr. Alessandro Torcini (Université de Cergy-Pontoise, France)
}

Promotionsausschuss:

Vorsitzender: Prof. Dr. Stephan Reitzenstein

Gutachter: Prof. Dr. Dr. h.c. Eckehard Schöll, PhD

Gutachter: PD Dr. Serhiy Yanchuk

Tag der wissenschaftlichen Aussprache: 24. September 2020

Berlin 2020 



\section{Abstract}

Collective phenomena in systems of interconnected dynamical units are omnipresent in nature. The swarm behavior in flocks of birds or schools of fish, the synchronous flashing of fireflies or even coherent spiking of neurons in the human brain are just a few examples of collective motion. Elucidating the mechanisms that give rise to synchronization is crucial in order to understand biological self-organization. For this sake, the theory of dynamical networks has been successfully applied over the last decades to boil down the complex dynamics from natural systems to their essentials. In addition, dynamical networks with adaptive couplings and hence non-constant network structure appear naturally in real-world systems such as power grid networks, social networks as well as neuronal networks. In this thesis, we study adaptive networks and their properties which give rise to the emergence of a variety of synchronization patterns, including complete and cluster synchronization as well as solitary and chimera-like states. One of the main fields of application that is investigated in this thesis concerns neuroscience. However, the methods and approaches developed in this work are not restricted to a specific field of application.

In the first part of this thesis, we report on the phenomenon of frequency clustering in globally coupled oscillator network with slow adaptation. As a motivating example, we study a network of Hodgkin-Huxley neurons with spike timing-dependent plasticity. Here, the clustering leads to a splitting of a neural population into a few groups synchronized at different frequencies. We propose a phenomenological model which describes the dynamics of two clusters taking the adaptive coupling weights into account. Following the successful application of the phenomenological model, we investigate a paradigmatic system of adaptively and globally coupled phase oscillators inspired by neuronal networks with synaptic plasticity. Our numerical as well as analytical study of the phase oscillator model allows for a complete description of the mechanism behind the emergence of frequency clustering. Moreover, we unveil the role of individual clusters for the shape and stability of frequency cluster (multiclusters), which explains the high level of multistability found in the paradigmatic model.

In the second part, we extend the findings from the first part towards complex network structures. We observe a variety of partially synchronized states such as phase-locked, multicluster, solitary and chimera-like states. Solitary states have been observed in a plethora of dynamical systems. However, the mechanisms behind their emergence were largely unaddressed in the literature. Here, we show how solitary states emerge in the model of phase oscillators due to the adaptive feature of the network and classify several bifurcation scenarios in which these states are created and stabilized. In addition, we investigate the stability of synchronous states for a large class of complex adaptively coupled oscillator networks. In particular, we generalize the master stability approach beyond the static network paradigm by taking adaptivity into account. We apply the new method to an adaptive network of coupled phase oscillators and show how the subtle interplay between adaptivity and network structure gives rise to the emergence of complex partial synchronization patterns.

In spite of the lively interest in the topic of adaptive networks, little is known about the interplay of adaptively coupled groups of networks. Such adaptive multilayer or multiplex networks 
appear naturally in neuronal networks. We propose a concept to generate and stabilize diverse partial synchronization patterns (phase clusters) in adaptive networks. We show that multiplexing induces various stable phase cluster states in a situation where they are not stable or do not even exist in the single layer. Further, we develop a method for the analysis of Laplacian matrices of multiplex networks which allows for insight into the spectral structure of these networks enabling a reduction to the stability problem of single layers. We employ the new method to provide analytic results for the stability of the multilayer patterns. 


\section{Zusammenfassung}

Kollektive Phänomene in Systemen von miteinander verbundenen dynamischen Einheiten sind allgegenwärtig in der Natur. Das Schwarmverhalten in Gruppen von Vögeln oder Fischen, das synchrone Blinken von Glühwürmchen oder auch das kohärente "Feuern" von Neuronen im menschlichen Gehirn sind nur einige Beispiele für kollektives Verhalten. Die Erforschung der Mechanismen, die zur Synchronisation führen, ist entscheidend für das Verständnis von biologischer Selbstorganisation. Zu diesem Zweck wurde in den letzten Jahrzehnten die Theorie der dynamischen Netzwerke erfolgreich angewandt, um die komplexen Bewegungen natürlicher Systeme auf das Wesentliche zu reduzieren. Darüber hinaus kommen dynamische Netzwerke mit adaptiven Kopplungen und damit nicht konstanter Netzwerkstruktur in realen Systemen wie Stromnetzwerken, sozialen Netzwerken sowie neuronalen Netzwerken ganz natürlich vor. In dieser Arbeit untersuchen wir adaptive Netzwerke und deren Eigenschaften, welche zur Entstehung einer Vielzahl von Synchronisierungsmustern, darunter vollständige und Cluster-Synchronisierung sowie solitäre und chimärenartige Zustände, beitragen. Eines der Hauptanwendungsgebiete, das in dieser Arbeit untersucht wird, sind die Neurowissenschaften. Die in dieser Arbeit entwickelten Methoden und Ansätze sind jedoch nicht auf ein bestimmtes Anwendungsgebiet beschränkt.

Im ersten Teil dieser Arbeit berichten wir über das Phänomen der Frequenz-Clusterung in global gekoppelten Oszillator-Netzwerken mit langsamer Adaptivität. Als motivierendes Beispiel untersuchen wir ein Netzwerk von Hodgkin-Huxley-Neuronen mit "spike timing-dependent" Plastizität. Hier führt die Clusterung zur Aufspaltung einer neuronalen Population in einige wenige Gruppen, die mit unterschiedlichen Frequenzen synchronisiert sind. Wir führen ein phänomenologisches Modell ein, das die Dynamik von zwei Clustern unter Berücksichtigung der adaptiven Kopplungsgewichte beschreibt. Nach der erfolgreichen Anwendung des phänomenologischen Modells untersuchen wir ein paradigmatisches System von adaptiv und global gekoppelten Phasenoszillatoren, das von neuronalen Netzwerken mit synaptischer Plastizität inspiriert ist. Unsere numerische sowie analytische Analyse des Phasenoszillatormodells ermöglicht eine vollständige Beschreibung des Mechanismus hinter der Entstehung von Frequenz-Clustern. Darüber hinaus stellen wir die tragende Rolle einzelner Cluster für die Form und Stabilität von Frequenz- Clustern (Multi-Cluster) heraus und erklären damit den hohen Grad an Multistabilität, der in dem paradigmatischen Modell zu finden ist.

Im zweiten Teil erweitern wir die Erkenntnisse aus dem ersten Teil auf komplexe Netzwerkstrukturen. Wir beobachten eine Vielzahl von partiell synchronen Zuständen, wie z.B. phasenstarre, Multi-Cluster-, solitäre und chimärenartige Zustände. Solitäre Zustände wurden in einer Vielzahl dynamischer Systeme beobachtet. Die Mechanismen, die hinter ihrer Entstehung stehen, sind in der Literatur jedoch weitgehend unbehandelt. Hier zeigen wir, wie solitäre Zustände in einem Model von Phasenoszillatoren aufgrund der adaptiven Eigenschaft des Netzwerks entstehen und klassifizieren mehrere Bifurkationsszenarien, in denen diese Zustände erzeugt und stabilisiert werden. Darüber hinaus untersuchen wir die Stabilität synchroner Zustände für eine große Klasse komplexer adaptiv gekoppelter Oszillator-Netzwerke. Insbesondere verallgemeinern wir den Master-Stabilitätsansatz über das statische Netzwerkparadigma hinaus, indem wir die Adaptivität berücksichtigen. Wir wenden die neue Methode auf ein adaptives Netzwerk 
aus gekoppelten Phasenoszillatoren an und zeigen, wie das subtile Zusammenspiel zwischen Adaptivität und Netzwerkstruktur die Entstehung komplexer partieller Synchronisationsmuster hervorruft.

Trotz des regen Interesses an adaptiven Netzwerken ist über das Zusammenspiel adaptiv gekoppelter Netzwerkgruppen wenig bekannt. Solche adaptiven Mehrschicht- oder MultiplexNetzwerke kommen in neuronalen Netzwerken ganz natürlich vor. Wir schlagen ein Konzept zur Erzeugung und Stabilisierung diverser partieller Synchronisationsmuster (Phasen-Cluster) in adaptiven Netzwerken vor. Wir zeigen, dass das "Multiplexen" verschiedene stabile PhasenCluster-Zustände induzieren kann, selbst wenn diese in den Einschicht-Systemen nicht stabil sind oder gar nicht existieren. Weiterhin entwickeln wir eine Methode zur Analyse von Laplace-Matrizen von Multiplex-Netzwerken, die einen Einblick in die spektrale Struktur dieser Netzwerke ermöglicht und damit eine Reduktion des Stabilitätsproblems auf die von EinschichtNetzwerken ermöglicht. Wir setzen die neue Methode ein, um analytische Ergebnisse für die Stabilität der Zustände im Mehrschicht-System zu erhalten. 


\section{Publications}

- [BER19]: R. Berner, E. Schöll, and S. Yanchuk: Multiclusters in networks of adaptively coupled phase oscillators, SIAM J. Appl. Dyn. Syst. 18, 2227 (2019).

- [BER19a]: R. Berner, J. Fialkowski, D. V. Kasatkin, V. I. Nekorkin, S. Yanchuk, and E. Schöll: Hierarchical frequency clusters in adaptive networks of phase oscillators, Chaos 29, 103134 (2019).

- [ROE19a]: V. Röhr, R. Berner, E. L. Lameu, O. V. Popovych, and S. Yanchuk: Frequency cluster formation and slow oscillations in neural populations with plasticity, PLoS ONE 14, e0225094 (2019).

- [BER19b]: R. Berner, J. Sawicki, and E. Schöll: Birth and stabilization of phase clusters by multiplexing of adaptive networks, Phys. Rev. Lett. 124, 088301 (2020).

- [BER20c]: R. Berner, A. Polanska, E. Schöll, and S. Yanchuk: Solitary states in adaptive nonlocal oscillator networks (2019), arXiv: 1911.00320. 



\section{Table of Contents}

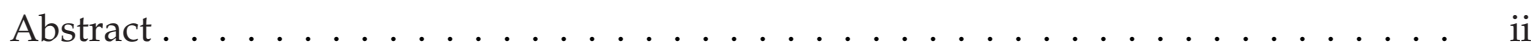

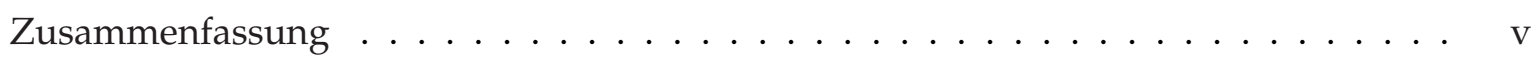

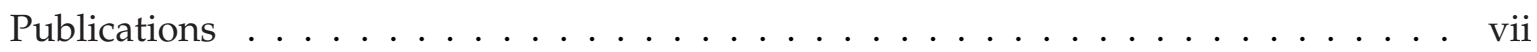

1 Introduction 1

1.1 Dynamics on complex networks . . . . . . . . . . . . . . . 1

1.2 Synchronization and collective phenomena . . . . . . . . . . . . . . . . . 2

1.3 Dynamics of complex networks . . . . . . . . . . . . . . 3

1.4 The role of phase oscillator models for complex dynamical networks . . . . . . 5

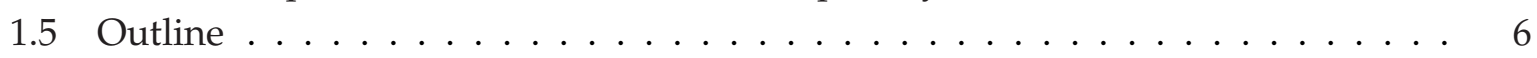

2 Fundamentals of adaptive and complex dynamical networks 9

2.1 Complex networks . . . . . . . . . . . . . . . . . . . 9

2.1.1 Networks, subnetworks, and connectivity . . . . . . . . . . . . . . 9

2.1.2 Special network types . . . . . . . . . . . . . . . . . 11

2.1 .3 Permutation symmetries in networks . . . . . . . . . . . . . . 13

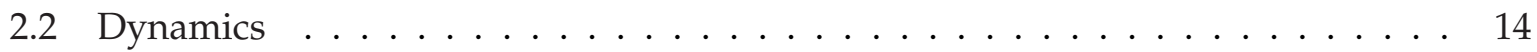

2.2 .1 Types of coupling . . . . . . . . . . . . . . . . . . . . . 14

2.2.2 Kuramoto-Sakaguchi type model . . . . . . . . . . . . . . . . . 15

2.2.3 Hodgkin-Huxley model with chemical synapses . . . . . . . . . . . . . . 17

2.3 Adaptive networks in neuroscience . . . . . . . . . . . . . . . . . . . . . . . . . . . 18

2.3.1 Spike timing-dependent plasticity . . . . . . . . . . . . . . . . . . . . . . 18

2.3.2 Phase difference-dependent plasticity . . . . . . . . . . . . . . . . . . . 19

2.3.3 A network of adaptively coupled phase oscillator . . . . . . . . . . . 20

2.4 Summary . . . . . . . . . . . . . . . . . . . . 21

\section{CLUSTER SYNCHRONIZATION IN GLOBALLY COUPLED ADAPTIVE NETWORKS 23}

3 Population of Hodgkin-Huxley neurons with spike timing-dependent plasticity 25

3.1 Coupled Hodgkin-Huxley neurons on a network with spike timing-dependent

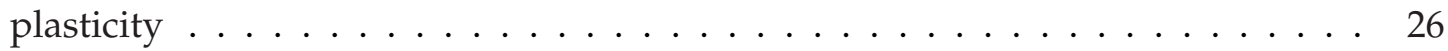

3.2 Numerical observation of synchrony and frequency clustering . . . . . . . . . 27

3.3 Emergence of two-cluster states . . . . . . . . . . . . . . . . . 30

3.4 Phenomenological model with phase difference-dependent plasticity . . . . . . . 34

3.4.1 Properties of the model . . . . . . . . . . . . . . . 36

3.4.2 Comparison of the model and cluster dynamics in Hodgkin-Huxley network 37

3.4.3 Criteria for the emergence of frequency clusters . . . . . . . . . . . . 37

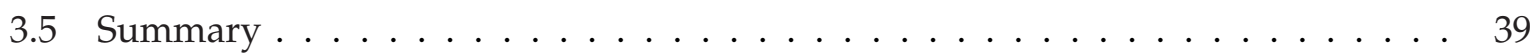


4 One-cluster states in adaptive networks of coupled phase oscillators 41

4.1 Classification of one-cluster states . . . . . . . . . . . . . . . . . . . . 41

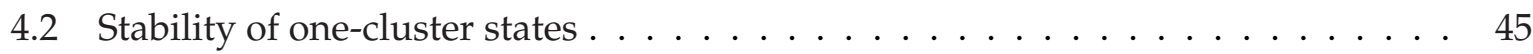

4.3 Adaptation rate dependence of one-cluster stability . . . . . . . . . . . . 50

4.4 Double antipodal states . . . . . . . . . . . . . . . . . . 52

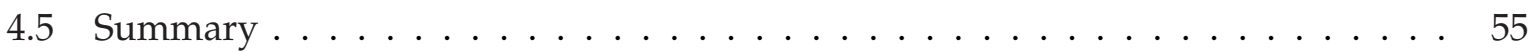

5 Multicluster states in adaptive networks of coupled phase oscillators 57

5.1 Numerical observation of multicluster states . . . . . . . . . . . . . . . . 58

5.1 .1 Splay type cluster states . . . . . . . . . . . . . . . . . . . . . 59

5.1 .2 Antipodal type cluster states . . . . . . . . . . . . . . . . 59

5.1 .3 Mixed type cluster states . . . . . . . . . . . . . . . . . . . . . 60

5.2 Splay type multicluster states . . . . . . . . . . . . . . . . . . 62

5.2.1 Conditions for the emergence of splay type multicluster states . . . . . . . 62

5.2 .2 Two-cluster states of splay type . . . . . . . . . . . . . . . . 63

5.2.3 Adaptation rate dependence for the emergence of two-cluster states . . . 65

5.3 Conditions for the emergence of multicluster states - A generalized approach . . 66

5.4 Antipodal type multicluster states . . . . . . . . . . . . . . . . . . . 69

5.4.1 Asymptotic conditions for the emergence of antipodal type multicluster states . . . . . . . . . . . . . . . . . . . . . . 69

5.4.2 Two-cluster states of antipodal type . . . . . . . . . . . . . 71

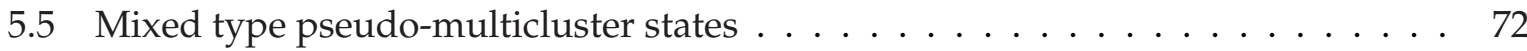

5.5.1 Asymptotic conditions for the emergence of mixed type pseudo-multicluster states . . . . . . . . . . . . . . . . . 72

5.5.2 Pseudo-two-cluster states of mixed type . . . . . . . . . . . . . . 73

5.6 Stability of multicluster states $\ldots \ldots \ldots \ldots \ldots \ldots \ldots \ldots$

5.6.1 On the stability of multicluster states with evenly sized clusters . . . . . 77

5.6.2 An effective approach for the stability of multicluster states . . . . . . . 78

5.7 Summary . . . . . . . . . . . . . . . . . . . . . . . 79

II INTERPLAY OF ADAPTIVITY AND CONNECTIVITY

6 Adaptation on nonlocally coupled ring networks 83

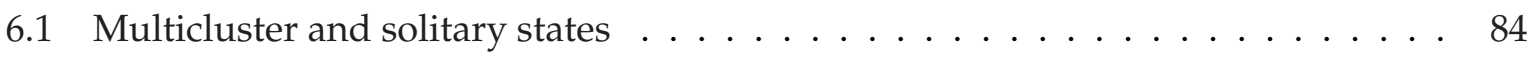

6.1.1 One-cluster states . . . . . . . . . . . . . . . . . . . . . . . . 85

6.1 .2 Multicluster states . . . . . . . . . . . . . . . . . . . . 86

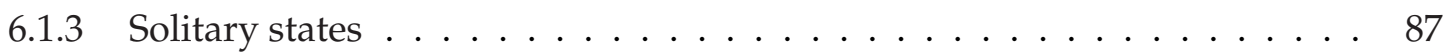

6.2 One-cluster states: Local vs. global features . . . . . . . . . . . . . . . . . . . 89

6.2.1 Classification of one-cluster states . . . . . . . . . . . . . . . . . . . 89

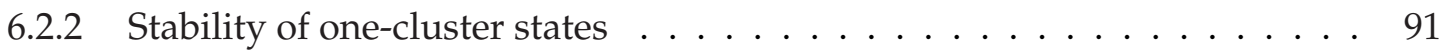

6.3 The emergence of solitary states . . . . . . . . . . . . . . . . . 93

6.4 Adaptive networks with global base topology versus ring base topology: the differences . . . . . . . . . . . . . . . . . . . . . . 96 
6.5 Summary . . . . . . . . . . . . . . . . . . . . 96

7 Synchronization on adaptive complex network structures 99

7.1 The master stability function for adaptive complex networks . . . . . . . . . . . 100

7.2 Stability islands in the presence of adaptation . . . . . . . . . . . . . . . . 104

7.3 Stability islands and implications for the emergence of multicluster states . . . 107

7.4 Summary . . . . . . . . . . . . . . . . . . . . . . . . . . . 109

8 Multilayered adaptive networks $\quad 111$

8.1 Lifted states in multiplex networks . . . . . . . . . . . . . . . . . . . . 112

8.2 Birth and robustness of phase clusters . . . . . . . . . . . . . . . 114

8.3 Multiplex decomposition . . . . . . . . . . . . . . . . . . . 117

8.4 Stabilizing through multiplexing . . . . . . . . . . . . . . . . 120

8.5 Applications for the multiplex decomposition . . . . . . . . . . . . . . . . . 122

8.5.1 The master stability approach for multiplex networks . . . . . . . . . . 122

8.5.2 Analytic treatment of diffusive dynamics on multiplex networks . . . . . 125

8.6 Summary . . . . . . . . . . . . . . . . . . . . 126

9 Conclusion and Outlook $\quad 127$

$\begin{array}{ll}\text { APPENDIX } & 133\end{array}$

A Proof of results from the main text 135

A.1 One-cluster states on globally coupled adaptive networks . . . . . . . . . . . . . 135

A.2 Stability of one-cluster states on globally coupled networks . . . . . . . . . . . 136

A.3 Multicluster states of splay type . . . . . . . . . . . . . . . . . . . . 142

A.4 Asymptotic expansions of multicluster states . . . . . . . . . . . . . . . . . . 142

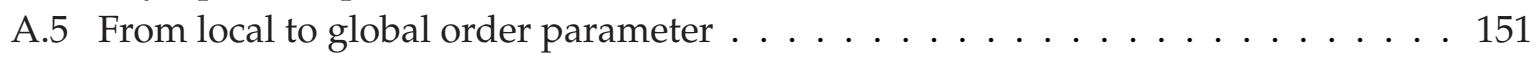

A.6 Stability of one-cluster states on nonlocally coupled networks . . . . . . . . . 152

A.7 Stability of lifted one-cluster states . . . . . . . . . . . . . . . . . . . 154

A.8 Example for a complex adjacency matrix . . . . . . . . . . . . . . . . 156

$\begin{array}{ll}\text { List of Figures } & 157\end{array}$

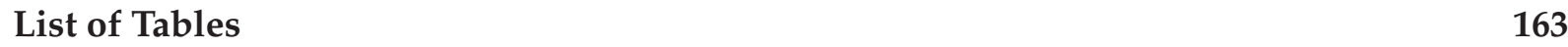

$\begin{array}{ll}\text { Acknowledgement } & 165\end{array}$

$\begin{array}{ll}\text { Bibliography } & 167\end{array}$ 



\subsection{Dynamics on complex networks}

Complex networks are an ubiquitous paradigm in nature and technology, with a wide field of applications from physics, chemistry, biology, neuroscience, as well as engineering and socioeconomic systems [NEW03]. A lot of work has been devoted to understand the statistical and topological properties of complex connectivity structures [ALB02a, COS07] ranging from random [ERD59, ERD60] and scale-free networks [BAR99a] to small-world structures[WAT98a] and even simplicial complexes that are topological structures used to model many body interactions [GIU16, SIZ19].

The field of complex dynamical networks studies the interplay of dynamics and (static) network structure [POR16a, BOC18]. Powerful methodologies as the Master Stability Function have been suggested to provide a unified approach to study synchronization of networks with arbitrary structure [PEC98, CHO09, KYR14, PEC14, WIL14, LEH15b].

The investigation of complex networks and their dynamical features has become a major research branch in neuroscience. Here, network neuroscience [BUL09, BAS17, BER19e] and neuronal dynamics [GER14a, BOE17a] complement each other in order to build up an understanding for functional and structural properties of the human brain. Within this context, various network structures equipped with different neuronal models have been studied over the last decades. For instance, ring-like structures are important motifs in neural networks [COM03, SPO11, POP11, YAN11]. Specifically, nonlocally coupled rings where each node is coupled to all nodes within a certain coupling range, are known to be important systems appearing in many applied problems and theoretical studies [PAS95, BRE97, YAN08a, BON09, ZOU09b, HOR09b, PER10c, OME11, KAN13, OME13, YAN15a, SCH16b, KLI17, BUR18, OME18a].

In addition to the nonlocally coupled networks, other coupling structures and motifs such as modular, scale-free, and small-world structures have been observed in the structural and functional connectivity of human brains [EGU05, HUM06, BAS06a, MEU10a, WIL12, RIE14, ASH19]. The interplay between neuronal dynamics and these structures already revealed important mechanisms in the functioning of dynamical brain networks [ZHO06c, ZHO07, BAS17, CHO18, HOE18, WAN19b, RAM19]. Despite this development and the flourishing interest in network neuroscience many questions are still unanswered or have not yet been asked [BAS17, BAS18].

Another focus of recent research in network science are multilayer networks, which are systems interconnected through different types of links [BOC14, DE13, DE15, KIV14]. A prominent example are social networks which can be described as groups of people with different patterns of contacts or interactions between them [GIR02, AMA17, AMA17a]. Other applications are communication, supply, and transportation networks, for instance power grids, subway networks, or airtraffic networks [CAR13d]. In neuroscience, multilayer networks represent for instance neurons in different areas of the brain, neurons connected either by a chemical link or by an electrical synapses [PER14a, MAJ18a, BER19d], or the modular connectivity structure 
of brain regions [BEN16, BAT17, VAI18]. A special case of multilayer networks are multiplex topologies, where each layer contains the same set of nodes, and only pairwise connections between corresponding nodes from neighboring layers exist [ZHA15a, MAK16, SEV16, JAL16, REQ16, GHO16, LEY17a, AND17, FRO18, PIT18, LEY18, GHO18, MIK18, SAW18, SAW18c, SEM18, OME19, RYB19, NIK19, BLA19, JAL19].

\subsection{Synchronization and collective phenomena}

Collective behavior in networks of coupled oscillatory systems has attracted a lot of attention over the last decades. Depending on the network and the specific dynamical system, various synchronization patterns of increasing complexity were explored. Even in simple models of coupled oscillators, patterns such as complete synchronization [KUR84, PIK01], cluster synchronization where the network splits into groups of synchronous elements [YAN01a, SOR07, BEL11a, DAH12, NIC13, PEC14, GOL16, ZHA20], and various forms of partial synchronization patterns have been found. The investigation of synchronization phenomena on complex networks [STR00, PIK01, STR01a, BOC02, STR03, NIS06, ARE08, BAL09, NEK15b, BOC18, MAI18] has become fruitful for applications in many areas ranging from physics and chemistry to biology, neuroscience, physiology, ecology, socio-economic systems, computer science and engineering. For example, synchronization of neurons is believed to play a crucial role in brain networks under normal conditions, for instance in the context of cognition and learning [SIN99, FEL11], and under pathological conditions such as Parkinson's disease [HAM07], epilepsy [JIR13, JIR14, ROT14, AND16], tinnitus [TAS12, TAS12a], schizophrenia, or autism, to name a few [UHL06]. Another example is the synchronized flashing in groups of fireflies [BUC68]. Also in power grid networks synchronization is essential for their operation [ROH12, MOT13a, MEN14, TAH19]. The control of dynamics on networks is another important issue with a lot of applications [SCH07, SCH16].

One of the most important partial synchronization patterns are chimera states. As they have been theoretically predicted [KUR02a, ABR04], these states are characterized by a spatial coexistence of coherence (synchronized domains) and incoherence (desynchronized domains). Numerous works have been devoted to this phenomenon, theoretically as well as experimentally, showing that chimera states play a crucial role for the understanding of real-world dynamical systems [MOT10, OME11, PAN15, YAO16, SCH16a, SCH16b, MAJ18a, OME18a, OME19c, SCH19c, ZAK20]. Apart from the theoretical importance, complex dynamical scenarios such as chimera states are found in a wide range of experimental systems, e.g. optical light modulators [HAG12] and chemical oscillators [TIN12, TOT15, TOT18], mechanical or optomechanical [MAR13, KAP14, OLM15, PEL20], electronic or optoelectronic oscillators [LAR13, LAR15], electrochemical systems [WIC13, WIC14, SCH14a], and electronic circuits [GAM14, ROS14a]. Further, it has been shown that chimera states are crucial for the understanding of epileptic seizures [AND16, CHO18] or unihemispheric sleep [RAT00, RAT16, RAM19]. Due to their importance, several approaches have been developed to control chimera states and other partial synchronization patterns [BIC15, OME16, OME18, RUZ19, SAW20].

Beyond chimera states various other types of partial synchronization scenarios have been found [PAZ05, ZAK14, MAI14a, ASH15, KLI15, ZHA15a, BI16]. Solitary [MAI14a] or Bellerophon states [BI16] are just two examples of the plethora of synchronization patterns discovered in 
the transition from incoherence to coherence or as a result of the destabilization of the coherent state.

Recently, solitary states have been found in diverse dynamical systems. Solitary states are described as states for which only one single element behaves differently compared with the behavior of the background group, i.e., the neighboring elements. These kinds of states have been observed in generalized Kuramoto-Sakaguchi models [MAI14a, WU18a, TEI19, CHE19b], the Kuramoto model with inertia [JAR15, JAR18], models of power grids [TAH19, HEL20], the Stuart-Landau model [SAT19], the FitzHugh-Nagumo model [RYB19a, SCH19a], systems of excitable units [ZAK16b] as well as in Lozi maps [RYB17] and even in experimental setups of coupled pendula [KAP14]. Solitary states are considered as important states in the transition from coherent to incoherent dynamics [JAR15, SEM15b, MIK18]. Despite their appearance in many well-studied models, the mechanisms of their emergence are less understood. Until now, only a few works could shed some light on the details behind their formation [MAI14a, JAR18, SEM18a, TEI19].

\subsection{Dynamics of complex networks}

Besides the paradigm of static networks the analysis of interconnected dynamical system on temporally evolving connectivity structures has become a future challenge in nonlinear science [POR19]. Here, two approaches have to be distinguished. The first which uses a prescribed temporal evolution of the network structure [HOL12, HOL15a], called temporal networks, and a second where the temporal evolution of the network depends upon on the dynamical state of the network nodes [GRO09], called adaptive networks. In this thesis, we focus on the latter approach and consider adaptive networks throughout.

With regard to this thesis, one of the main motivations for studying adaptive dynamical networks comes from the field of neuroscience. Here, a possible mechanisms that causes adaptation, which may lead to persistent changes in neural connections and relates to learning and memory, is synaptic plasticity [HEB49]. The efficiency of synapses to transmit the electrical potential between neurons may increase or decrease depending on the mutual neural activity, which results in short- or long-term potentiation or, respectively, depression of synapses [BRO88a, BLI93]. An example is spike timing-dependent plasticity (STDP) which describes the change of the synaptic weight as a function of the difference of spiking times between pre- and post-synaptic neurons [GER96, MAR97a, BI98, ABB00, BI01, CAP08a, MEI09a, LUE16]. Thus the network structure reorganizes adaptively in response to the neuronal dynamics. Similarly, chemical systems have been reported [JAI01], where the reaction rates adapt dynamically depending on the variables of the system. Activity-dependent plasticity is also common in epidemics [GRO06b] and in biological or social systems [GRO08a]. Many studies are devoted to the mechanism of plasticity in chemical synapses, see e.g. [ABB00, MAR11b], however, it has been shown that also electrical synapses undergo plastic changes [MER14, PER14a].

A famous example for a plasticity rule is the Hebbian rule which assumes that the modifications of the synaptic weights are driven by correlations in the firing activity of pre- and post-synaptic neurons. More specifically, the rule assumes that those connections are potentiated, for which one neuron contributes to the firing of another [HEB49]. Nevertheless, in many publications, 
the Hebbian rule is considered in a narrower sense of closeness between the spiking times: the smaller the distances between the spikes are the higher is the potentiation of the corresponding synapse [HOP96, SEL02]. In this work, we are dealing with spike-based learning rules rather than rate-based.

Previous studies of neural networks with STDP (or STDP-like) showed that such networks can evolve and create various coupling and spiking patterns [CAT08, MIL13b, MIK14]. For instance, the coupling weights can exhibit stable localized spatial structures, which can be interpreted as receptive fields [CLO10]. These structures can be either unidirectionally of bidirectionally coupled, depending on the plasticity rule or external input properties. The STDP mechanism plays an important role in temporal coding of information by spikes [GER96, CLO10]. On the one hand, synchronized firing in neural ensembles with STDP can be stabilized through potentiation of synaptic coupling by stimulation-induced transient synchronization of neurons [TAS06, TAS12a, POP13, LUE16]. On the other hand, a desynchronized state can lead to a depression of synaptic weights [TAS06, TAS12a]. Thus, neural networks with plasticity are prone to coexistence of different stable dynamical and structural states, which may be realized by choosing appropriate initial conditions or stimulation procedures.

In addition to the localized spatial structures, the emergence of modular and scale-free networks has been reported [ITO01a, ITO03, STA10b, GUT11, ASS11, YUA11, AOK12, WIN12, AOK15a, BOT12, BOT14, POP15, CHA17a] in networks with STDP. This fact underlines the potential importance of adaptive mechanism in the formation of connectivity structures as they have been experimentally found in brain networks [MEU10a, ASH19]. Furthermore, activity based adaptive rewiring has even been shown to enhance modularity [RUB09].

Besides direct application to neuroscience, spike timing-dependent plasticity rules have been discussed for neurocomputing [HOP99] and have recently also been implemented into memristive devices [DU15, JOH18]. For early work on electronic switching and S-shaped current-voltage characteristics see [SCH87, SHA92, SCH01]. Memristors and memristor arrays play an important role in the development of neuromorphic computing [PIC13, WAL13, IGN15, HAN17, BIR19] which is believed to be the future of artificial intelligence. An exhaustive survey on approaches to neuromorphic computing is presented in Ref. [SCH17k] showing the plethora of work devoted to this questions. The huge common interest of the scientific community in developing braininspired technologies is not least expressed by the flagship project "The human brain project" funded by the European Union [MAR12c, AMU19] or the NIH BRAIN Initiative [KOR18a]. Therefore, there is clearly an urge for the analysis of adaptive neural networks and the development of novel methods to understand these systems.

Next to the active interest in adaptive networks with regards to neuroscience, motivation for systems with adaptive connectivity structure has been found in other dynamical systems from biology [PAI14] and social science [SAY13, SAY15, AOK16], in systems that describe swarming behavior of coupled agents [IWA10, IWA10a], or in the dynamics of communication networks [GAV12]. Furthermore, it has been shown that synchronization in complex networks is enhanced by an adaptation of the coupling structure [ZHO06f, ZHU10]. Due to this, adaptive network structures have been also successfully applied to control synchronization in complex and even time-evolving networks [LEL08, SOR08, WAN08f, LEL09, LEL10, LEL10a, SCH12, SEL12, GUZ13, LEH14, PLO16]. 


\subsection{The role of phase oscillator models for complex dynamical networks}

Models of coupled phase oscillators, i.e., models where the dynamical variable is simply given by an angle, are nowadays a well-known paradigm in order to study collective behavior of dynamical oscillatory units on complex networks [ACE05, PIK01]. In this context, a particularly important feature is that coupled nonlinear oscillators can be reduced to phase oscillator networks in case of weak interactions [WIN80, HOP97, PIK01, PIE19a]. The reduction of complex dynamical systems to networks of coupled phase oscillators is well-known and there exist exhaustive reviews highlighting the importance of phase oscillator models [PIK01, ASH16, PIE19a]. Recent studies, in addition, aim to make phase oscillators models even more powerful by generalizing the conditions under which the reduction techniques are valid [KLI17a, ROS19a, ROS19b, ERM19]

As a representative from the class of phase oscillator models, the Kuramoto model where all oscillators are coupled in an all-to-all manner, particularly, has attracted a lot of attention due to its simple form and mathematical tractability [KUR84, STR00]. The Kuramoto model has gained additional popularity due to its application for real-world problems [STR93, PIK01, STR03, STR05a, ROD16]. Despite the simple structure of the Kuramoto model many different dynamical regimes have been observed. In order to get a hand on the dynamics of the Kuramoto model sophisticated methods have been developed. In particular, sinusoidally and globally coupled phase oscillators have been shown to be partially integrable. The Watanabe-Strogatz theory, which was introduced to show the integrability, allows for a reduction of a system with finite size to only three dimensions and can be applied to even more general classes of phase oscillator models [WAT93a, WAT94, STE11b]. Another approach which has been developed to understand coupled oscillators in the continuum limit is the Ott-Antonson ansatz [OTT08]. The Ott-Antonson theory allows for a reduction to a two dimensional dynamical system in the case of an infinite number of oscillators. The theory has been successfully applied to describe the emergence of chimera states even beyond phase oscillator models [OME08, ABR08, LAI09, OME13, OME18a, OME19c]. Remarkably for both reduction techniques, the Watanabe-Strogatz and the Ott-Antonson theory, the reduced systems possess a clear physical interpretation, and both approaches are intimately related with each other [MAR09c, PIK15]. Just recently, another very promising reduction technique for finite systems has been introduced which make use of so-called collective coordinates [HAN18b, SMI19, SMI20].

Beyond the classical Kuramoto model various generalization have been proposed. Starting from generalization to complex networks [GOM07, DOE14], the theory of phase oscillators has been further developed to study phenomena of phase transitions [PAZ05a, GOM11a, BOC16], network symmetries [NIC13], the impact of inertia [ERM91, FIL08a, MAI14a, SCH14m, OLM15a] and other forms of frequency adaptation [TAY10], delayed coupling [YEU99a], or the effect of time-depending parameters [PET12] to name just a few.

Another generalization which gained a lot of attention over the last years concerns the effects and phenomena in networks of phase oscillators with adaptive coupling. Various models have been suggested and studied to gain insights into the interplay of collective dynamics and adaptivity [MAS17c]. A lot of works are inspired by recent findings in neuroscience and implement synaptic plasticity into their models [SEL02, REN07, MAI07, MAS07c, AOK09, NIY09, TAK09, 
LI10c, AOK11, GUT11, ASS11, SKA13a, CHA14a, REN14, TIM14, AOK15, HA16a, KAS16a, NEK16, ASL17, KAS17, ASL18, ASL18a, KAS18a, KAR19]. Despite the rich interest in neuroscientific applications, also other forms of adaptation and other applications have been considered. For example, effects of adaptive rewiring, an adaptive network growth, or evolutionary edgesnapping are studied in Refs. [GLE06, LI11a, SCA15, PAP17, DAM19]. Also the interplay of adaptivity and complex network structure such as multilayers have been only recently numerically investigated [MAK16a, KAS18, KAS19].

\subsection{Outline}

This thesis is devoted to the analysis of partial synchronization patterns in complex oscillator networks with adaptive coupling strength. This analysis is organized in two parts. After two introductory chapters, in the first part, the Hodgkin-Huxley neuron model and the phase oscillator model on a globally coupled network with synaptic plasticity are investigated. Here, we observe the emergence of multiclusters due to plasticity and underline the importance of phase oscillator models in order to understand these complex synchronization pattern. By rigorous mathematical methods, we provide a comprehensive understanding of the cluster structures. In part II, the paradigmatic model of adaptively coupled phase oscillators, introduced in part I, is used in order to study the interplay of adaptivity and complex connectivity structures.

The basic formalism and the standard notation which is used through out part I and II is presented in Chapter 2. Here, we provide graph theoretical preliminaries and introduce all classes of networks studied in this thesis. Furthermore, a brief introduction into the dynamics on complex networks is given where we discuss different types of coupling and models. In the last section, we show how adaptivity is modeled in the context of synaptic plasticity. In addition, the reduction to phase oscillator models with phase difference-dependent plasticity is given.

Part I starts with Chapter 3. In this chapter, we analyze a population of Hodgkin-Huxley neurons with spike timing-dependent plasticity. The numerical observation of multiclusters is shown and the detailed mechanisms of the stability of frequency clusters is explained by using the simplest case of two clusters. Here, we propose a phenomenological model, which describes the dynamics of two clusters taking into account the adaptation of the weights. The model is shown to reflect not only qualitative, but also some basic quantitative properties of the two-cluster formation. We also determine the set of plasticity functions (update rules), which lead to the clustering.

In Chapter 4, the existence and stability of one-cluster states is rigorously analyzed in the phase oscillator model. We provide a full classification of one-cluster states and investigate the impact of the timescale separation on their stability. Different types are characterized by different phase relations of the nodes. Finally, we discuss the role of a special type of oneclusters, called double antipodal states. Although such states are unstable for all parameter values, they appear as saddles connecting synchronous and splay states. As a result, double antipodal states are observed during a "meta-stable" transition between the phase-synchronous and non-phase-synchronous state.

Chapter 5 is devoted to the phenomenon of multicluster states which are distinguished by different frequencies of the individual clusters. The multiclusters are, first, illustrated using 
numerical integration, and then classified. Complementing earlier work, we find a mixed multicluster state. These new multiclusters consist simultaneously of splay and (in-) anti-phase synchronous states. It results from this that all one-cluster states can serve as building blocks for a multicluster. Further, we provide an analytic description of all types of multicluster states using explicit methods as well as methods from the theory of asymptotic expansions. We show that multiclusters corresponding to certain fixed frequency ratio appear in continuous families, and, moreover, multiclusters with different frequency ratios coexist for the same parameter values. We derive algebraic equations for the frequencies of coexisting multicluster solutions. In a particular case of two clusters, these equations can be explicitly solved. In the end, the stability of multicluster states is discussed and connected to the stability of their individual building blocks, i.e., one-cluster states.

The second part starts with Chapter 6. In this chapter, we analyze the emergence of solitary states on a nonlocally coupled ring in the presence of plastic coupling weights. For this, numerical results and a rigorous definition for multicluster and solitary states on complex networks are presented. We further provide a detailed analysis of one-cluster states and derive explicit relations between local and global properties. A reduced model for two-clusters is derived which allows us to unveil the bifurcation scenarios in which solitary states are formed and (de)stabilized.

Complex networks of adaptively coupled oscillators are studied in Chapter 7. Here, we consider the stability of synchronous states and derive the master stability function for a large class of adaptive networks. The new method is applied to a system of adaptively coupled phase oscillators with complex connectivity structure. We observe the existence of stability islands in the master stability function and connect these islands to the emergence of multicluster as well as chimera-like states.

In chapter 8 we show that a plethora of novel patterns can be generated by multiplexing adaptive networks in a multilayer configuration. In particular, partial synchronization patterns like phase clusters and more complex cluster states which are unstable in the corresponding monoplex network can be stabilized, or even states which do not exist in the single-layer case for the parameters chosen, can be born by multiplexing. We elucidate the delicate balance of adaptation and multiplexing which is a feature of many real-world networks even beyond neuroscience.

The thesis concludes with Chapter 9 where the obtained results are summarized and discussed. Furthermore, an outlook on possible continuations of this work is given. 



\section{Fundamentals of adaptive and complex dynamical networks}

Throughout this thesis well-established mathematical and physical concepts are used and extended in order to describe dynamical systems with complex and adaptive coupling structure. In this chapter, we fix the notation and introduce the basic formalism that is used in the subsequent considerations.

The chapter is organized as follows. In Section 2.1, we provide graph theoretical preliminaries and introduce all classes of networks studied in this thesis. Furthermore, a brief introduction into dynamics on complex networks is given in Section 2.2 where we discuss different types of coupling and models. In the Section 2.3, we show how adaptivity is modeled in the context of synaptic plasticity. In addition, the reduction to phase oscillator models with phase differencedepend plasticity is given.

\subsection{Complex networks}

This section is devoted to the introduction of graph theoretical concepts for networks and the description of special classes of networks that will be considered in this thesis. Here, we follow standard textbooks and literature on graph theory and complex networks [GOD01, COS07, NEW10].

\subsubsection{Networks, subnetworks, and connectivity}

In network science the notions graph and network, vertex and node, and edge and link, respectively, are used interchangeably. For the sake of clarity, we use the notions network, node, and link. In general we define a directed network $\mathcal{N}$ as a triple $\mathcal{N}=(V, E, \Psi)$ of finite sets $V$ and $E$, and $\Psi: E \rightarrow\{(v, w) \in V \times V\}$. Elements from the sets $V$ and $E$ are called nodes and links, respectively. The total number of nodes of a network is denote by $N=|V|$. Usually the range of the map $\psi$ is restricted to $\{(v, w) \in V \times V: v \neq w\}$ in order to avoid nodes to be connected to themselves. However, in this thesis self-coupling is allowed for all further consideration. Note that for an undirected network $\Psi: E \rightarrow\{X \subseteq V:|X| \in\{1,2\}\}$ which means that $\psi$ maps the edge $e \in E$ to $X=v$ if the mapping $\psi(e)$ refers to self-coupling or it maps the edge to $X=\{v, w\}$ if $\psi(e)$ refers to an undirected link between node $v$ and $w(v \neq w)$.

By definition, two links can be mapped through $\Psi$ to the same element of $V \times V$. If this is the case, i.e., there exist $e, e^{\prime} \in E$ such that $\psi(e)=\psi\left(e^{\prime}\right)$, the network is called a multinetwork (multigraph is more commonly used). If on the contrary all links can be uniquely identified with their images under the map $\Psi$, the network is called a simple network. This latter type of networks build the backbone of hundreds of studies on complex networks and forms the basis of the subsequent chapters. Note that for simple networks with $V=\left\{v_{1}, \ldots, v_{N}\right\}$ every link $e$ can be assigned to its image $\Psi(e)$ which allows for the shorthand notation $e_{i j}$ as the link connecting two nodes starting at node $v_{j}$ and ending at node $v_{i}(i, j=1, \ldots, N)$. In this case the map $\Psi$ can be omitted 
in the definition of a network. For the sake of simplicity, we refer to simple networks as networks unless stated differently. Further, we associate an undirected network $\mathcal{N}^{\prime}=\left(V^{\prime}, E^{\prime}\right)$ to a directed network $\mathcal{N}=(V, E)$ by $V^{\prime}=V, E^{\prime}=\left\{e_{i j}: e_{i j} \in E\right.$ or $\left.e_{j i} \in E, i, j=1, \ldots, N\right\}$. Note that it is also possible to assign a directed network to an undirected network by introducing an orientation of the links [GOD01].

Another important notion which will be used in this thesis is the induced subnetwork. A subnetwork of a network $\mathcal{N}$ is given by $\mathcal{N}^{\prime}=\left(V^{\prime}, E^{\prime}, \Psi\right)$ if $V^{\prime} \subseteq V$ and $E^{\prime} \subseteq E$. In addition, a subnetwork is denoted by induced if $E^{\prime}=\left\{e_{i j} \in E: v_{i}, v_{j} \in V^{\prime}\right\}$.

The connectivity structure for a simple network is often represented by an $N \times N$ matrix $A$, called adjacency matrix, which possesses the entries

$$
a_{i j}= \begin{cases}1, & \text { if } e_{i j} \in E, \\ 0, & \text { otherwise }\end{cases}
$$

This algebraic view on networks by representing their structure in form of a matrix is the one we use throughout this work [CVE97, GOD01, NEW10]. This viewpoint allows one to define the in-degree $d(i)$ of node $v_{i}$ by considering the row sum of the adjacency matrix, i.e.,

$$
d(i)=\sum_{j=1}^{N} a_{i j} .
$$

Further, we define the Laplacian matrix $L$ of a network as

$$
L=\left(\begin{array}{cccc}
d(1) & 0 & \cdots & 0 \\
0 & \ddots & \ddots & \vdots \\
\vdots & \ddots & \ddots & 0 \\
0 & \cdots & 0 & d(N)
\end{array}\right)-A .
$$

Note that this matrix is a discrete version of the well-known Laplacian operator known from, e.g., the theory of waves. In particular, for certain dynamical networks such as nonlocally coupled rings with diffusive coupling, as they are defined later on in Section 2.2, the relation between the discrete and the continuum version of the Laplacian can be shown explicitly [KOU14, ISE15, ISE15a].

A $v_{i}-v_{j}$ walk of length $L \in \mathbb{N}$ on a network is a sequence $v_{i}, e_{k_{1} i}, v_{k_{1}}, \ldots, e_{j k_{L-1}}, v_{j}$ for $v_{i}, v_{j} \in V$ and $e_{k_{m} k_{m-1}} \in E$ for all $m=1, \ldots, L$ where $k_{0}=i$, and $k_{L}=j$. With this, a network is said to be connected (or weakly connected [KOR18]) if for any two nodes $v_{i}, v_{j} \in V\left(v_{i} \neq v_{j}\right)$ exists at least one $v_{i}-v_{j}$ walk on the associated undirected network. Note that according to this definition, two nodes $v_{i}$ and $v_{j}$ are connected if either $e_{i j} \in E$ or $e_{j i} \in E$. We can utilize the adjacency matrix in order to analyse whether there is a $v_{i}-v_{j}$ walk of length $L$ between two nodes. For this, we consider the $L$ th power of the adjacency matrix for the associated undirected network and find that there exists a $v_{i}-v_{j}$ walk if $\left(A^{L}\right)_{j i} \neq 0$. Therefore, a network is connected if the matrix $\sum_{l=1}^{N} A^{l}$ possesses no vanishing entry. The spectrum of the Laplacian matrix, also called graph spectrum, allows for another way to find whether a network is connected. Note that by definition any Laplacian matrix possesses a zero eigenvalue which belongs to the eigenvector $(1, \ldots, 1)^{T}$. 

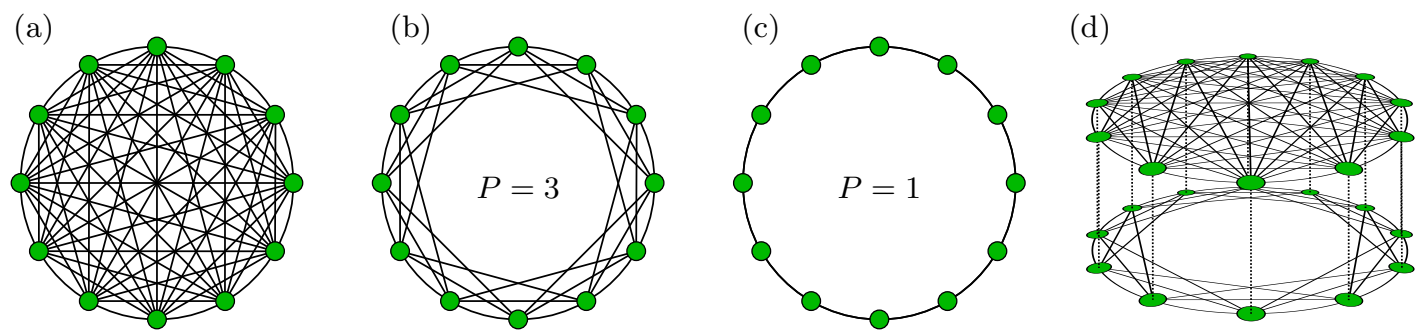

Figure 2.1: Illustration of different networks with a total number of 12 nodes: (a) globally coupled network, (b) nonlocally coupled network (coupling range $P=3$ ), (c) locally coupled network (coupling range $P=1$ ), and $(\mathrm{d}$ ) multiplex network consisting of two layers for which one layer is globally coupled and the other layer has a nonlocally coupled network structure.

Further, for undirected networks the Laplacian is symmetric and therefore its spectrum consist of eigenvalues such that $0=\lambda_{1}(L) \leq \lambda_{2}(L) \leq \cdots \leq \lambda_{|V|}(L)$. The second smallest eigenvalues is called algebraic connectivity (sometimes denoted as Fiedler number) and vanishes if and only if the network is unconnected [FIE73, FIE89].

Lastly, for each network we can define a link weighting $\Xi: E \rightarrow \mathbb{R}$ which assigns real numbers (weights) to each link of the network. According to this map $\Xi$, the weight matrix $\kappa$ with entries

$$
\kappa_{i j}= \begin{cases}\Xi\left(e_{i j}\right), & \text { if } e_{i j} \in E, \\ 0, & \text { otherwise }\end{cases}
$$

With regards to dynamical networks, the weight matrix is also called coupling matrix and its entries coupling weights.

In the following we describe several types of networks with special structural properties.

\subsubsection{Special network types}

\section{Globally coupled network}

The simplest network is the globally coupled network (also complete or all-to-all network) which consist of $N$ nodes where each node is connected to every other node. Hence, the entries of the adjacency are given by

$$
a_{i j}= \begin{cases}1, & \text { if } i \neq j, \\ 0, & \text { otherwise, }\end{cases}
$$

if there is no self-coupling, otherwise $a_{i j}=1$ for all $i, j=1, \ldots, N$. An illustration is provided in Fig. 2.1(a).

The spectral properties of this type of network are easily determined. The Laplacian for a globally coupled network possesses, besides the single zero eigenvalue, the $N$-1-times degenerate eigenvalue $N$. 


\section{Circulant networks and nonlocally coupled rings}

Another important and already well-studied network structure is represented by circulant adjacency matrices, i.e., where each row vector $\boldsymbol{a}_{i}=\left(a_{i 1}, \ldots, a_{i N}\right)$ is rotated one element to the right relative to the preceding row vector [DAV79, GRA05]. These adjacency matrices correspond to a ring network where each element has the same coupling structure. For this type of networks the spectrum can be determined by using the discrete Fourier transformation. In particular, the discrete Fourier vectors $(l=1, \ldots, N)$

$$
\zeta_{l}=\frac{1}{\sqrt{N}}\left(e^{\mathrm{i} \frac{2 \pi l}{N}}, \ldots, e^{\mathrm{i} \frac{2 \pi(N-1) l}{N}}, 1\right)^{T}
$$

are the $N$ eigenvectors for the $N$ corresponding eigenvalues

$$
\lambda_{l}=\sum_{m=1}^{N} a_{1 m} e^{\mathrm{i} \frac{2 \pi m l}{N}} .
$$

A particular case of circulant structures are nonlocally coupled ring networks. These networks are given by

$$
a_{i j}= \begin{cases}1, & \text { if }(i-j) \quad \bmod N \leq P, \\ 0, & \text { otherwise, }\end{cases}
$$

where $P$ denotes the so-called coupling range. Illustrations for these networks are shown in Fig. 2.1(b,c). In Fig. 2.1(b), we show a nonlocally coupled ring network for $P=3$. Figure 2.1(c) displays a locally coupled ring network with coupling range $P=1$ where each node is only coupled to its direct neighbor. By changing the coupling range between $P=1$ and $P=N / 2$ (if $N$ is even, else $P=(N+1) / 2$ ) the corresponding network structure range from the locally coupled to the globally coupled network, respectively.

Another intriguing circulant network structure arises in the context of fractal connectivities. Here, a Cantor construction algorithm has been used to derive iteratively a vector $\boldsymbol{a}$ from a base pattern [HIZ15, OME15, PLO16a, TSI16, ULO16, KRI17, SAW19].

\section{Multilayer and multiplex networks}

Multilayer networks are networks where the whole set of nodes is divided into subsets of nodes which are said to belong together. The induced subnetworks for the individual subsets are then called layers. From the mathematical point of view, multilayer networks are just networks. However, the special structure of a multilayer network, i.e., the partition into several subsets, has recently been considered very important in order to describe the dynamics on real-world networks. For exhaustive reviews on multilayer networks and their mathematical description we refer to Refs. [DOM13, BOC14, KIV14]. In the following, we discuss some features of multilayer networks by considering the special class of multiplex networks.

Multiplex networks are a particular kind of multilayer networks. These networks consist of $L \in \mathbb{N}$ layers of $N$ nodes where the connectivity structure within the layer is given by $L$, generally 
different, adjacency matrices $A^{\mu}(\mu=1, \ldots, L)$. These adjacency matrices determine the intralayer networks structure. Let $\operatorname{Per}(N)$ denote the set of all $N \times N$ permutation matrices [LIE15]. Then, the connectivity between the layers is given by $\left(L^{2}-L\right)$ matrices $I^{\mu v} \in \operatorname{Per}(N) \cup \hat{0}$ where $\hat{0}$ is the zero matrix. Hence, all nodes of one layer are either uniquely identified with all nodes of another layer via the inter-layer structure $I^{\mu \nu}$ or there are no connection between the layers. An illustration of a multiplex networks with two layers is provided in Fig. 2.1(d). Here, the two layers are bidirectionally connected with $I^{12}=I^{21}=\mathbb{I}_{N, N}$. Within the layers, however, the coupling structure is different. One layer possesses a globally coupled while the other layer possesses a nonlocally coupled ring intra-layer structure.

An algebraic representation of multilayer networks is achieved by multilinear forms [DOM13]. By flattening of these tensor structures, however, a representation via an adjacency matrix is obtained. Here flattening means that one can relate a finite dimensional tensor space to another finite dimensional vector space via an isomorphism, see [KIV14]. In this context the representation is called supra-adjacency matrix and takes the following block matrix form

$$
A=\left(\begin{array}{cccc}
A^{1} & I^{12} & \ldots & I^{1 L} \\
I^{21} & \ddots & \ddots & \vdots \\
\vdots & \ddots & \ddots & I^{(L-1) L} \\
I^{L 1} & \cdots & I^{L(L-1)} & A^{L}
\end{array}\right) .
$$

Usually the inter-layer structures are assumed to be given by the identity matrix $\mathbb{I}_{N, N}$.

The spectral properties of multiplex networks have been firstly discussed in [GOM13, SOL13a]. Here, results based on perturbation for weighted multiplex networks in case of weak and strong inter-layer coupling have been derived. As a part of the present thesis, novel results on the spectrum of multiplex networks are provided in Chapter 8.

\subsubsection{Permutation symmetries in networks}

Here, we introduce the notion of symmetry for networks. Symmetries are always described as special transformations leaving certain structures of interest invariant. For this, in case of networks, permutations of nodes are considered as symmetry transformation. A permutation of nodes of a network is given by the endomorphism $\Pi: V \rightarrow V, v_{i} \mapsto v_{\pi(i)}$ with $\pi \in S_{N}$ and $i=1, \ldots, N$. By these means, we call such a transformation a symmetry if the following holds: $e_{i j} \in E$ if and only if $e_{\pi(i) \pi(j)} \in E$. Transformations with this property, also called automorphisms of a network, form a group, so-called automorphism group or symmetry group, under the composition [BOL98]. The symmetry group of a network $\mathcal{N}$ is denoted by $\operatorname{Aut}(\mathcal{N})$.

Symmetries appear in many real-world systems [MAC08]. Utilizing these symmetry properties the analysis of the spectral properties [MAC09a] of complex networks can be simplified, complex networks can be reduce to simpler structure [XIA08]. Moreover, certain scenarios in the spatial and temporal dynamics of systems are found to be induced by network symmetry [FIE88, GOL88a, ASH92b]. 


\subsection{Dynamics}

In dynamical networks all nodes and links of a weighted network $\mathcal{N}$ represent dynamical entities and their weighted interaction with each other, respectively. Formally, a dynamical network of $N$ nodes equipped with dynamical $d$-dimensional variables $\boldsymbol{x}_{i} \in \mathbb{C}^{d}(i=1, \ldots, N)$, as they are analyzed throughout this thesis, may be written as follows:

$$
\dot{\boldsymbol{x}}_{i}=f_{i}\left(\mathbf{x}_{i}(t)\right)-\sigma \sum_{j=1}^{N} a_{i j} \kappa_{i j} G_{i j}\left(\boldsymbol{x}_{i}, \boldsymbol{x}_{j}\right),
$$

where $f_{i} \in C^{1}\left(\mathbb{C}^{d}, \mathbb{C}^{d}\right)$ describes the local dynamics of node $i$, the functions $G_{i j} \in C^{1}\left(\mathbb{C}^{d}, \mathbb{C}^{d}\right)$ determine the pairwise coupling between the nodes $i$ and $j$. Here, $C^{1}\left(\mathbb{C}^{d}, \mathbb{C}^{d}\right)$ denotes the set of differentiable functions $f: \mathbb{C}^{d} \rightarrow \mathbb{C}^{d}$ with continuous derivative. The basic coupling structure is given by the matrix entries $a_{i j} \in\{0,1\}$ of the $N \times N$ adjacency matrix $A$. Each existing link is additionally weighted by the coupling weights $\kappa_{i j}$. The parameter $\sigma \in \mathbb{R}$ is the overall coupling constant.

In the previously described framework of dynamical systems only pairwise interactions are considered. Note that there exist generalizations in order to describe many-body interaction by using, for instance, simplicial complexes which are topological generalization of "classical" networks [TAN11b, ASH16b, BIC16, SKA19, LEO19]. In generalizations using simplicial complexes, interactions between nodes are not only described by links (1-simplex) but also by higher $n$-dimensional $n$-simplices that can be thought of as the convex hull of $(n+1)$ vertices, see [GIU16] for an introduction. However, these models are beyond the scope of this work.

\subsubsection{Types of coupling}

In real world systems, single dynamical units are not isolated but interact by in some cases very complicated interaction mechanism. For the purpose of describing these interactions between the units, simplified interaction schemes given by the coupling function $G$ have been developed. In the following, we introduce the most important schemes which are used in the subsequent chapters

In the context of diffusive systems on complex networks, linear diffusive processes were considered in order to study the dynamics on social as well as transport networks [BAR11d, GOM13]. Moreover, also in neuronal populations the coupling through electrical synapses (gap-junctional connections) is modeled by a linear diffusive map [BEN04a, KOP04, CON04, CUR16, REI17a, ALC19]. The described form of coupling can be formally written as

$$
G_{i j}\left(x_{i}, x_{j}\right)=G_{i j}\left(x_{i}-x_{j}\right)
$$

where the coupling functions becomes $d \times d$ matrices $\boldsymbol{G}_{i j}$. Usually the coupling scheme is assumed to be identical for all nodes, i.e., $\boldsymbol{G}_{i j}=\boldsymbol{G}$ for all $i, j=1, \ldots, N$. In the case of an identical coupling scheme, the input which the $i$ th node receives via the interaction with the other nodes of the network is given by the sum $s(i)=\sum_{j=1}^{N} a_{i j} \boldsymbol{G}\left(\boldsymbol{x}_{i}-\boldsymbol{x}_{j}\right)$. Here, $a_{i j}$ are the entries of the adjacency matrix of the network. Remarkably, this sum can be written as $s(i)=\sum_{j=1}^{N} l_{i j} \boldsymbol{G} \boldsymbol{x}_{j}$ where 
$l_{i j}$ are the entries of the Laplacian matrix $L$, see (2.3). This fact allows for the relation between dynamical properties and the Laplacian structure of a network. It has been used, for instance, to introduce the powerful methodology of the master stability function [PEC98, LEH15b] .

Linear coupling is in some sense the simplest type of coupling. More complex forms of diffusive couplings can be written as

$$
G_{i j}\left(x_{i}, x_{j}\right)=G_{i j}\left(x_{i}-x_{j}\right),
$$

where $G_{i j}$ are nonlinear functions depending on the difference $\boldsymbol{x}_{i}-\boldsymbol{x}_{j}$. For example, in the well-known Kuramoto model $G_{i j}$ is given by a sin function [KUR84]. Other forms of nonlinear coupling schemes, not necessarily diffusive, are used to model interactions in various contexts, see for instance Refs. [TRU13, PEN19, BAU20]. Note that despite the nonlinear coupling scheme the corresponding variational equations around each state are still linear and diffusive. Hence, stability features depend on the Laplacian structure of the network, as well.

Very elaborate types of coupling schemes are used in models of neurons that exhibit inhibitory coupling through chemical synapses. Here, the coupling is usually assumed to be nonlinear with a slow synaptic decay. The coupling through chemical synapses is modeled by a first order kinetics of the form

$$
\begin{aligned}
G_{i j}\left(V_{i}, V_{j}\right) & =s_{j} \cdot\left(V_{i}-V_{r}\right) \\
\dot{s}_{j} & =\frac{\alpha_{s}}{1+e^{-\left(V_{j}-\Theta_{s}\right) / \sigma_{s}}}\left(1-s_{j}\right)-s_{j} / \tau_{s}
\end{aligned}
$$

where $V_{i}$ is the membrane potential of neuron $i, V_{r}$ is the reverse potential, $s_{j}$ is the gating variable describing the synaptic transmission from the $j$ th neuron, $\tau_{s}$ is the synaptic decay time constant. Further, the parameter $\alpha_{s}, \Theta_{s}$, and $\sigma_{s}$ characterize the sigmoidal part in the dynamics of the gating variables [WAN92, WHI98, HAU09, POP13, POP15]. Depending on which process is causing the interaction of neurons, other models for chemical coupling can be found similar to the one described [RIN90, GIL04].

Building on these different types of coupling, we introduce the dynamical network models which are used in this thesis.

\subsubsection{Kuramoto-Sakaguchi type model}

A rather simple model, known as Kuramoto-Sakaguchi type model, describes a dynamical network of $N$ coupled phase oscillators

$$
\frac{d \phi_{i}}{d t}=\omega_{i}-\frac{1}{N} \sum_{j=1}^{N} a_{i j} \kappa_{i j} \sin \left(\phi_{i}-\phi_{j}+\alpha\right),
$$

where $\phi_{i} \in T^{N}, T^{N}$ denoting the $N$-torus, represents the phase of the $i$ th oscillator $(i=1, \ldots, N)$ and $\omega_{i}$ its natural frequency. The interaction is the same between each pair of phase oscillators and given by a sinusoidal coupling kernel. The weighted network is represented by the entries of the adjacency matrix $a_{i j}$ and the coupling weigths $\kappa_{i j}$. The parameter $\alpha$ can be considered as a phase-lag of the interaction [SAK86]. System (2.14) has attracted a lot of attention over the last 
three decades since it is a first choice paradigmatic model for the modeling of synchronization and partial synchronization scenarios on complex dynamical networks [KUR84, STR00, ACE05, OME12b, PIK08].

Coherence (phase synchronization) in a network of phase oscillators can be measured by the so-called Kuramoto-Daido order parameter [KUR84, DAI92a, DAI94]. The $n$th order parameter for the state $\phi \in \mathbb{T}^{N}$ is defined as

$$
Z_{n}(\boldsymbol{\phi})=R_{n}(\boldsymbol{\phi}) e^{\mathrm{i} \theta_{n}(\boldsymbol{\phi})}=\frac{1}{N} \sum_{j=1}^{N} e^{\mathrm{i} n \phi_{i}},
$$

where $n \in \mathbb{N}$. The symbols $R_{n}>0$ and $\theta_{n} \in \mathbb{T}^{1}$ denote the modulus and the phase of the complex order parameter, respectively. The number $n$ can be also called moment of the order parameter. Note that the symbol i denotes the imaginary unit to distinguish it from the index $i$. In case of full phase synchronization, i.e., $\phi=(a, \ldots, a)^{T}$ for any $a \in T$, the modulus of the order parameter $R_{n}=1$. More generally, if for any $n \in \mathbb{N}$ the phase of the $i$ th oscillator is given by $\phi_{i} \in\{a, a+2 \pi / n, a+4 \pi / n, \ldots, a+2(n-1) \pi / n\}$ for some $a \in T$ then $R_{n}=1$. Thus, $R_{n}$ is a measure for a particular type of coherence with respect to a certain discrete phase distribution. Hence, the classical Kuramoto order parameter $Z_{1}$ measures in-phase synchrony. If $R_{n}=0$ we consider the phase distribution as incoherent with respect to the $n$th moment.

A measure that can be used in order to detect frequency synchronization between two oscillators relies on the average frequency of each phase oscillator

$$
\Omega_{i}=\lim _{T \rightarrow \infty} \frac{1}{T}\left(\phi_{i}\left(t_{0}+T\right)-\phi\left(t_{0}\right)\right)
$$

and the frequency synchronization measure is given by

$$
\Omega_{i j}=\left\{\begin{array}{l}
1, \text { if } \Omega_{i}-\Omega_{j}=0, \\
0, \text { otherwise }
\end{array}\right.
$$

see e.g. [ASH15]. Numerically the limit is approximated by very long average window. In addition, we use a sufficiently small threshold $\varpi$ in order to detect frequency synchronization numerically, i.e., $\Omega_{i j}=1$ if $\Omega_{i}-\Omega_{j}<\varpi$. In the subsequenty analysis we used $\varpi=0.001$. Using the measure $\Omega_{i j}$, we may define the following value which we call cluster parameter

$$
R_{C}=\frac{1}{N^{2}} \sum_{i, j=1}^{N} \Omega_{i j}
$$

The cluster parameter measures the following. First, for each frequency cluster, the total number of links connecting nodes of the same cluster is computed. In other word, how much space is occupied by a single cluster. Second, all ratios are summed up and normalized by the number of all possible links. In case of full frequency synchronization, i.e., $\Omega_{i j}=1$ for all $i, j=1, \ldots, N$ then $R_{C}=1$ because the single cluster "occupies" the whole network. A similar measure be found in Ref [KAS18a]. In the context of phase oscillator many different measures were introduced to characterize the dynamical states. For an overview and a comparison of different coherence measures for complex networks of phase oscillators see [SCH17m]; see also [MEH17a, MEH18] 
where a similar generalized order parameter has been suggested independently.

\subsubsection{Hodgkin-Huxley model with chemical synapses}

A more realistic model for neuronal dynamics is given by the Hodgkin-Huxley model [HOD52, GER14a]. Nowadays there exists a zoo of neuron models describing different features of neural cells. Due to its rich dynamics the Hodgkin-Huxley model is, however, one of the most prominent neuron models but also one of the most computationally expensive [IZH04]. In this work we are interested in neurons with tonic spiking dynamics. These kinds of neurons are excitable neurons which show a periodic spiking pattern in case of a constant synaptic input [IZH04]. A network of $N$ excitatory Hodgkin-Huxley neurons with inhibitory coupling is described by the following system [HOD52, HAN93b, POP13, LUE16]

$$
\begin{aligned}
C \dot{V}_{i} & =I_{i}-g_{N a} m_{i}^{3} h_{i}\left(V_{i}-E_{N a}\right)-g_{k} n_{i}^{4}\left(V_{i}-E_{K}\right)-g_{L}\left(V_{i}-V_{r}\right)-\frac{\left(V_{i}-V_{r}\right)}{N} \sum_{j=1}^{N} \kappa_{i j} s_{j}, \\
\dot{m}_{i} & =\alpha_{m}\left(V_{i}\right)\left(1-m_{i}\right)-\beta_{m}\left(V_{i}\right) m_{i}, \\
\dot{h}_{i} & =\alpha_{h}\left(V_{i}\right)\left(1-h_{i}\right)-\beta_{h}\left(V_{i}\right) h_{i}, \\
\dot{n}_{i} & =\alpha_{n}\left(V_{i}\right)\left(1-n_{i}\right)-\beta_{n}\left(V_{i}\right) n_{i}, \\
\dot{s}_{i} & =\frac{5\left(1-s_{i}\right)}{1+e^{\left(\frac{-V_{i}+3}{8}\right)}}-s_{i} .
\end{aligned}
$$

Here $V_{i}$ is the membrane potential of the $i$ th neuron with the corresponding equilibrium potentials $E_{N a}=50 \mathrm{mV}, E_{K}=-77 \mathrm{mV}$, and $E_{l}=-54.4 \mathrm{mV} . C=1 \mu \mathrm{F} / \mathrm{cm}^{2}$. Our choice of the reverse potential $V_{r}=20 \mathrm{mV}$ corresponds to the excitatory neurons. The three other variables $m, h$, and $n$ are gating variables in natrium and potassium, and their dynamics depends on the opening and closing rates

$$
\begin{aligned}
\alpha_{m}(V) & =\frac{0.1 V+4}{1-e^{(-0.1 V-4)}}, \\
\beta_{m}(V) & =4 e^{\left(\frac{-V-65}{18}\right)}, \\
\alpha_{h}(V) & =0.07 e^{\left(\frac{-V-65}{20}\right)}, \\
\beta_{h}(V) & =\frac{1}{1+e^{(-0.2 V-3.5)}}, \\
\alpha_{n}(V) & =\frac{0.01 V+0.55}{1-e^{(-0.1 V-5.5)}}, \\
\beta_{n}(V) & =0.125 e^{\left(\frac{-V-65}{80}\right)} .
\end{aligned}
$$

The parameters are $g_{N a}=120 \mathrm{mS} / \mathrm{cm}^{2}, g_{K}=36 \mathrm{mS} / \mathrm{cm}^{2}$, and $g_{l}=0.3 \mathrm{mS} / \mathrm{cm}^{2}$. The constant current $I_{i}$ is set to $9 \mu \mathrm{A} / \mathrm{cm}^{2}$ so that the individual neurons are identical and oscillatory. Note that we usually assume no autapses and set $\kappa_{i i}=0$. 


\subsection{Adaptive networks in neuroscience}

A dynamical network is called an adaptive network if its topological structure is non-static and depends on the state of the dynamical nodes. In this sense, the topological structure is determined by either the coupling matrix $\kappa(t)$ or the adjacency matrix $A(t)$. We note at this point that adaptive networks are to distinguish from temporal networks which possess a non-static connectivity structure, as well, but whose temporal dynamics is independent from the state of the nodes [HOL12, HOL15a].

Adaptive networks appear in many real-world systems [GRO08a, GRO09, SAY13]. In the framework of this thesis, the most important example of this class of dynamical systems are given by (tonically) spiking neurons with synaptic plasticity. Synaptic plasticity, in neural systems, is induced by various different interdependent mechanisms [FEL12]. We focus on the plasticity which is induced by the timing of spikes, so called spike timing-dependent synaptic plasticity (STDP). In addition, we consider plasticity of chemical synapses only. Note, however, that plastic changes in the connectivty through electrical synapses have been also reported [LAN05a, MAT14]. Note that in the description of the large-scale structure of the human brain exist completely other models of plasticity. These models, for instance, assimilate probabilistic forms of neuronal plasticity and do not rely on the precise timing of the neuron [GER14a, BRE17a]. The interplay of all forms of plasticity plays certainly a crucial role in structure formation, however, the analysis of this interplay is beyond the scope of this thesis. In the following, we briefly introduce the models used in order to describe STDP. For more details on the modeling and the physiological properties, we refer the reader to the reviews [ABB00, DAN06, LET07, CAP08a, SJO08, FRO10, MAR11b].

\subsubsection{Spike timing-dependent plasticity}

The synaptic input current from $j$ th presynaptic to $i$ th postsynaptic neuron is scaled by the synaptic weight $\kappa_{i j}$, see for example (3.1). The coupling weights $\kappa_{i j}$ have been shown to be a variable entity. Adaptation of $\kappa_{i j}$ due to the spike timings of neurons occurs discontinuously whenever one of the neurons $i$ or $j$ spikes. More specifically, the discontinuous change is formally given by the following plasticity function

$$
\kappa_{i j} \rightarrow \begin{cases}0, & \text { if } \kappa_{i j}+\delta W\left(\Delta t_{i j}\right)<0 \\ \kappa_{i j}+\epsilon W\left(\Delta t_{i j}\right), & \text { if } 0 \leq \kappa_{i j}+\delta W\left(\Delta t_{i j}\right) \leq \kappa_{\max } \\ \kappa_{\max ,} & \text { if } \kappa_{i j}+\delta W\left(\Delta t_{i j}\right)>\kappa_{\max }\end{cases}
$$

where $\Delta t_{i j}=t_{i}-t_{j}$ is the spike time difference between the postsynaptic and presynaptic neurons; $\epsilon>0$ is a small parameter determining the size of the single update; and $\kappa_{\max }>0$ is the maximal coupling [GER96].

In neuronal systems various forms of spike timing-depending plasticity exist depending for example on the type of synapse or the type of the postsynaptic neuron [BI98]. An overview on the diversity of plasticity rules has been presented in [SHU13]. In the subsequent chapter 3, we particularly consider the plasticity function [GER96, BEL97a]

$$
W\left(\Delta t_{i j}\right)=c_{p} e^{-\frac{\left|\Delta t_{i j}\right|}{\tau_{p}}}-c_{d} e^{-\frac{\left|\Delta t_{i j}\right|}{\tau_{d}}}
$$


with positive parameters $c_{p}, \tau_{p}, c_{d}$, and $\tau_{d}$.

\subsubsection{Phase difference-dependent plasticity}

Models which show self-sustained limit cycle oscillations are often used to describe excitatory neurons [IZH03, IZH04, ASH16]. In the context of these models a neuron is said to spike, and thus releases an action potential, if the dynamical variable which describes the membrane potential reaches its maximum.

It is well-known that networks of such oscillators interacting with each other can be reduced to dynamical networks of weakly coupled phase oscillators [HAN93b, HOP96a, PIE19a]. In this framework a neuron is said to spike whenever the neuron's phase equals zero. In order to reduce the complexity of neuronal networks with STDP it is desirable to establish a reduced model of coupled phase oscillators accompanied by synaptic plasticity which depends rather on the phase than the spike time difference. For this model of synaptic plasticity, Lücken et. al. introduced the notion "phase difference-dependent plasticity" (PDDP) and drew a relation between STDP and PDDP [LUE16]. Such a PDDP rule is written as

$$
\dot{\kappa}_{i j}=\epsilon h\left(\phi_{i}-\phi_{j}\right) .
$$

The relation between STDP and PDDP is derived as follows. Consider the spiking time of a postsynaptic $t_{\text {post }}$ and presynaptic $t_{\text {pre }}$. the spiking time difference $\Delta t=t_{\text {post }}-t_{\text {pre }}$ is thus always positive or negative (or zero) if the postsynaptic or the presynaptic neuron spikes (if the neurons spike synchronously), respectively. Consider now the phase difference $\theta=\phi_{\text {pre }}-\phi_{\text {post }}$ and assume that the phase difference changes much slower than the phase dynamics. Then by averaging the synaptic effect induced by a spike of the post and presynaptic neuron and by the assumption that the chances due to the plasticity are sufficiently small, we get the following continuous PDDP

$$
h(\theta)=\frac{\Omega}{2 \pi}\left[W_{+}\left(\frac{\theta}{\Omega}\right)+W_{-}\left(\frac{\theta-2 \pi}{\Omega}\right)\right]
$$

where $\Omega / 2 \pi$ is the number of spike per unit of time, $W_{+}=W(\Delta t)$ for $\Delta t>0$, and $W_{-}=W(\Delta t)$ for $\Delta t \leq 0$ ( $\Delta t \leq 0$ if $W$ is discontinuous) [LUE16]. Note that this adaptation rule does not guarantee that the values of the coupling weights are bounded during the course of time. This is why they have to be forced to stay within a bounded interval $\left[\kappa_{\min }, \kappa_{\max }\right]$ by either by a hard-cut as presented in (2.21) or by a soft-cut given as

$$
\dot{\kappa}_{i j}=-\frac{2 \epsilon}{\left(\kappa_{\max }-\kappa_{\min }\right)(\bar{\kappa}-\underline{\kappa})}\left((\bar{\kappa}-\underline{\kappa}) \kappa_{i j}-\left(\kappa_{\max }-\kappa_{\min }\right) h\left(\phi_{i}-\phi_{j}\right)+\kappa_{\max } \underline{\kappa}-\kappa_{\min } \bar{\kappa}\right)
$$

where $\bar{\kappa}=\max _{\theta \in[0,2 \pi]} h(\theta)$ and $\underline{\kappa}=\min _{\theta \in[0,2 \pi]} h(\theta)$. This soft-cut rule is obtained by normalization. Another rule using coupling weight dependent scaling of the plasticity function has been used in [MAI07]. In the following, we use a simplified PDDP in order to introduce a paradigmatic model for adaptively coupled phase oscillators. 

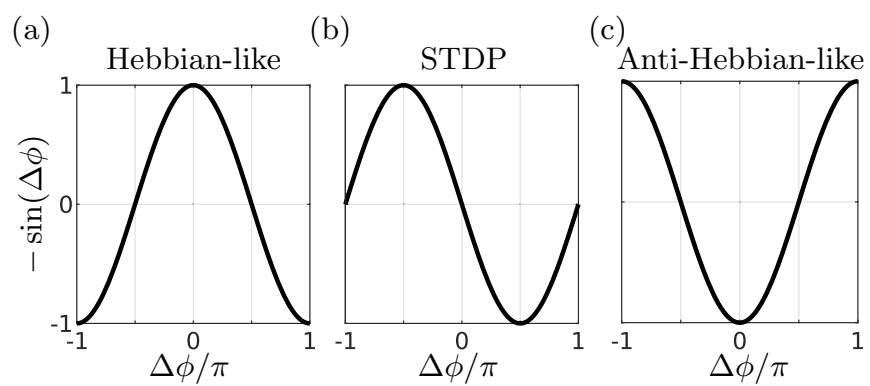

Figure 2.2: The plasticity function $-\sin (\Delta \phi+\beta)$ and corresponding plasticity rules. (a) $\beta=-\frac{\pi}{2}$, (b) $\beta=0$, (c) $\beta=\frac{\pi}{2}$. Figure taken from [BER19, BER19a].

\subsubsection{A network of adaptively coupled phase oscillator}

In the following we describe the model which is in the focus of this thesis. By introducing a PDDP, we introduced a differential equation which governs the dynamics of the coupling weights, see Eq. (2.23). In addition, the adaptation function $h$ is a $2 \pi$-periodic that can expanded in a Fourier series. Consider an adaptive networks of $N$ adaptively coupled phase oscillators as given by (2.14) with PDDP of the form (2.24) where $h$ is approximated by the first Fourier mode and we restrict the coupling weights to $[-1,1]$. Then, the dynamical networks reads

$$
\begin{aligned}
\frac{d \phi_{i}}{d t} & =\omega-\frac{1}{N} \sum_{j=1}^{N} \kappa_{i j} \sin \left(\phi_{i}-\phi_{j}+\alpha\right), \\
\frac{d \kappa_{i j}}{d t} & =-\epsilon\left(\kappa_{i j}+\sin \left(\phi_{i}-\phi_{j}+\beta\right)\right),
\end{aligned}
$$

The phase space of the system $(\phi(t), \kappa(t)) \in \mathbb{T}^{N} \times[-1,1]^{N^{2}}$ is $\left(N+N^{2}\right)$-dimensional. System (2.25)-(2.26) have attracted a lot of attention recently [REN07, AOK09, AOK11, PIC11a, TIM14, GUS15a, KAS16a, NEK16, KAS17, AVA18], since it is a first choice paradigmatic model for the modeling of the dynamics of adaptive networks. In particular, it generalizes the Kuramoto (or Kuramoto-Sakaguchi) model with fixed $\kappa$.

The matrix $\kappa(t)$ characterizes the coupling topology of the network at time $t$. Assume that $\epsilon$ is a small but not vanishing parameter. Then, the dynamical equation (2.26) describes the adaptation of the network topology depending on the dynamics of the network nodes. The chosen adaptation function in the form $\sin \left(\phi_{i}-\phi_{j}+\beta\right)$ with control parameter $\beta$ can take into account different plasticity rules that can occur in neuronal networks, see Fig. 2.2. For instance, for $\beta=-\pi / 2$, the Hebbian rule is obtained where the coupling $\kappa_{i j}$ is increasing between any two systems with close phases, i.e., $\phi_{i}-\phi_{j}$ close to zero [HEB49, HOP96, SEL02, AOK15]. If $\beta=0$ the link $\kappa_{i j}$ will be strengthened if the $j$-th oscillator is advancing the $i$-th. Such a relationship is typical for spike-timing-dependent plasticity in neuroscience [CAP08a, MAI07, LUE16, POP13].

Let us mention important properties of the model. Firstly, the parameter $\epsilon \ll 1$ separates the time scales of the slow dynamical behavior of the coupling strengths and the fast dynamics of the oscillatory system [KUE15]. Due to the invariance of system (2.25)-(2.26) with respect to the phase-shift $\phi_{i} \mapsto \phi_{i}+\psi$ for all $i=1, \ldots, N$ and $\psi \in \mathbb{T}^{1}$, the frequency $\omega$ can be set to zero in the co-rotating coordinate frame $\phi \mapsto \phi+\omega t$. Moreover, one can restrict the consideration of 
the coupling weights to the interval $-1 \leq \kappa_{i j} \leq 1$ due to the existence of the attracting region $G:=\left\{\left(\phi_{i}, \kappa_{i j}\right): \phi_{i} \in \mathbb{T}^{1},\left|\kappa_{i j}\right| \leq 1, i, j=1, \ldots, N\right\}$ [KAS17].

Finally, let us mention the parameter symmetries of the model

$$
\begin{aligned}
\left(\alpha, \beta, \phi_{i}, \kappa_{i j}\right) & \mapsto\left(-\alpha, \pi-\beta,-\phi_{i}, \kappa_{i j}\right), \\
\left(\alpha, \beta, \phi_{i}, \kappa_{i j}\right) & \mapsto\left(\alpha+\pi, \beta+\pi, \phi_{i},-\kappa_{i j}\right) .
\end{aligned}
$$

As a result of these symmetries one can restrict the analysis to the parameter region $\alpha \in[0, \pi / 2)$ and $\beta \in[-\pi, \pi)$.

\subsection{Summary}

In this chapter, we have given the theoretical framework which is used throughout the subsequent chapters. References for further reading are provided and the most important results for different topics are highlighted. We have first introduced notations and concepts from graph theory in order to have a proper description of weighted networks. Several examples for complex network structures are presented and some of their topological properties are reviewed. The notion of symmetry in networks is briefly introduced.

After this, we have used the concept of networks to define systems of coupled dynamical units. We have described how the links of a network relate to the coupling dynamics of nodes and what kind of coupling functions can be found in physical applications. Subsequently, a Kuramoto-Sakaguchi-like and the Hodgkin-Huxley model are introduced. These models are used throughout this thesis. Particularly, for the phase oscillator model, we have presented measures which allow for the quantitative description of synchronization in the system.

Extending the concepts from coupled dynamical units on static networks, we have described how adaptivity is modeled in the context of neuroscience. We have introduced spike timingdependent plasticity which is used to describe changes of the synaptic coupling weights dependent on the spike-timing of the neurons. Under the assumption of topically spiking neuron, neuronal systems with spike-timing dependent plasticity rules can be reduced to phase oscillators models with phase difference-dependent plasticity. We have shown how this is done and introduced a paradigmatic model for adaptively coupled phase oscillators with synaptic plasticity. 



\section{Part I}

\section{Cluster SYNCHRONIZATION IN GLOBALLY COUPLED ADAPTIVE NETWORKS}





\section{Population of Hodgkin-Huxley neurons with spike timing-dependent plasticity}

In this chapter we report on the phenomenon of clustering with respect to connectivity and frequencies in a network of adaptively coupled Hodgkin-Huxley neurons. The spike timingdependent plasticity is considered to be symmetric as it was experimentally found for hippocampal synapses [WIT06] and derived from asymmetric STDP for an "effective time window" [KEP02]. In this case the observed clusters are bidirectionally coupled [POP15]. Splitting of a neural population to a few clusters that are synchronized at different frequencies could lead to a slow waxing and waning of the amplitude of the mean field, where the clusters transiently gather together and move apart as the time evolves [POP15]. The frequency of such a modulation of the mean neural activity could be much smaller than the firing rate of individual neurons and depends on the differences between the clusters' frequencies. The emergence of synchronized clusters could explain the origin of the low-frequency modulation of the power spectral density of macroscopic brain signals like local field potentials (LFP) or electroencephalographic (EEG) signals in higher frequency bands, which also correlates with slow oscillations of the blood oxygen level-dependent (BOLD) signal measured by fMRI [MAN07, MAG12a, MON08, ALV14]. Several other modeling studies have also reported on clustering in the neural populations with plasticity [MAI07, CAT08, POP15]. These clusters have been observed for different models that ranged from simple phase oscillators to the models of spiking and bursting neurons and demonstrate stability with respect to heterogeneity of the interacting neurons and random perturbations [MAI07, CAT08, POP15]. In this chapter we provide a simple phenomenological model and explain a mechanism governed by synaptic plasticity for the stabilization of such clusters in a neural population.

This chapter includes contents that have been published in [ROE19a] and is structured as follows. In the first Section 3.1 we present the model. The next Section 3.2 shows numerically observed multiclusters. The detailed mechanisms of the stability of frequency clusters is explained afterwards in Section 3.3 using the simplest case of two clusters. Then, in Section 3.4, we propose a phenomenological model which describes the dynamics of two clusters taking into account the adaptation of the weights. The model is shown to reflect not only qualitative, but also some basic quantitative properties of the two-cluster formation. We also determine the set of plasticity functions (update rules), which lead to the clustering. The findings of this chapter are summarized in Section 3.5. 


\subsection{Coupled Hodgkin-Huxley neurons on a network with spike timing-dependent plasticity}

For the present study we consider $N$ coupled Hodgkin-Huxley neurons which are described by the dynamical equations

$$
\begin{aligned}
C \dot{V}_{i} & =I_{i}-g_{N a} m_{i}^{3} h_{i}\left(V_{i}-E_{N a}\right)-g_{k} n_{i}^{4}\left(V_{i}-E_{K}\right)-g_{L}\left(V_{i}-V_{r}\right)-\frac{\left(V_{i}-V_{r}\right)}{N} \sum_{j=1}^{N} \kappa_{i j} s_{j}, \\
\dot{m}_{i} & =\alpha_{m}\left(V_{i}\right)\left(1-m_{i}\right)-\beta_{m}\left(V_{i}\right) m_{i}, \\
\dot{h}_{i} & =\alpha_{h}\left(V_{i}\right)\left(1-h_{i}\right)-\beta_{h}\left(V_{i}\right) h_{i}, \\
\dot{n}_{i} & =\alpha_{n}\left(V_{i}\right)\left(1-n_{i}\right)-\beta_{n}\left(V_{i}\right) n_{i}, \\
\dot{s}_{i} & =\frac{5\left(1-s_{i}\right)}{1+e^{\left(\frac{-V_{i}+3}{8}\right)}}-s_{i} .
\end{aligned}
$$

Each of the neurons $(i=1, \ldots, N)$ is described by the membrane potential $V_{i}$ and the three gating variables $m_{i}, h_{i}$, and $n_{i}$. The parameters are chosen such that each individual neuron gives rise to self-sustained oscillatory dynamics. For more details, we refer to (2.19)-(2.20) in Chapter 2.

The interaction between the neurons is given by the synaptic input current $s_{j}$ which each neuron receives from the $j$ th neuron. the input current is scaled by the synaptic strength $\kappa_{i j}$, which we assume to change due to plasticity. The adaptation of $\kappa_{i j}$ occurs discontinuously whenever one of the neurons $i$ or $j$ spikes. More specifically, the discontinuous change is given by the following plasticity function, see also (2.21)

$$
\kappa_{i j} \rightarrow \begin{cases}0, & \text { if } \kappa_{i j}+\delta W\left(\Delta t_{i j}\right)<0 \\ \kappa_{i j}+\delta W\left(\Delta t_{i j}\right), & \text { if } 0 \leq \kappa_{i j}+\delta W\left(\Delta t_{i j}\right) \leq \kappa_{\max } \\ \kappa_{\max }, & \text { if } \kappa_{i j}+\delta W\left(\Delta t_{i j}\right)>\kappa_{\max }\end{cases}
$$

where $\Delta t_{i j}=t_{i}-t_{j}$ is the spike time difference between the postsynaptic and presynaptic neurons; $\delta>0$ is a small parameter determining the size of the single update; $\kappa_{\max }>0$ is the maximal coupling; and the plasticity function [GER96, MAR97a, BI98, CLO10] is

$$
W\left(\Delta t_{i j}\right)=c_{p} e^{-\frac{\left|\Delta t_{i j}\right|}{\tau_{p}}}-c_{d} e^{-\frac{\left|\Delta t_{i j}\right|}{\tau_{d}}}
$$

with positive parameters $c_{p}, \tau_{p}, c_{d}$, and $\tau_{d}$. We also assume no autapses and set $\kappa_{i i}=0$.

An example of the considered plasticity function $W$ used in our simulations is shown in Fig. 3.1. This is a symmetric function, which corresponds to a potentiation of the coupling weights of the neurons with highly correlated firing. As we will discuss at the end of this chapter, there is a family of plasticity functions of similar form that allow for the frequency clustering. 


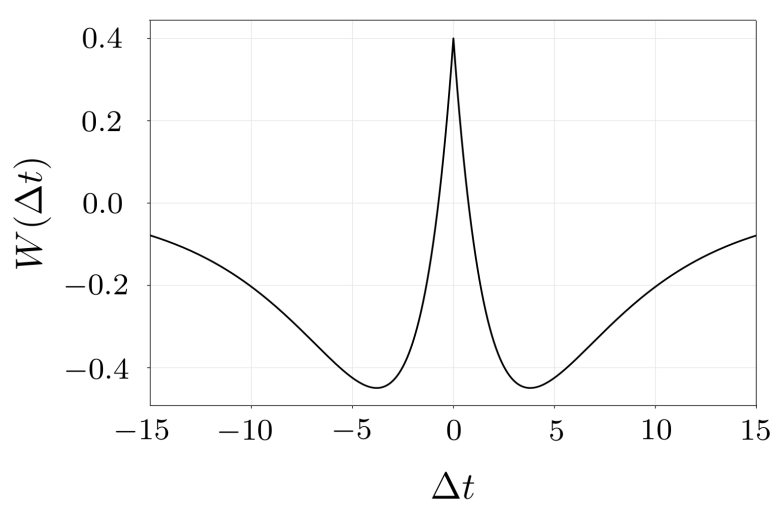

Figure 3.1: Plasticity function $W\left(\Delta t_{i j}\right)$ for $\tau_{p}=2, \tau_{d}=5, c_{p}=2, c_{d}=1$.6. Figure taken from [ROE19a].

\subsection{Numerical observation of synchrony and frequency clustering}

In order to investigate the dynamics of the network (3.1) with plasticity 3.3, we initialize the neurons and the coupling randomly and integrate the system numerically. For the parameter values $\tau_{p}=2, \tau_{d}=5, c_{p}=2, c_{d}=1.6$, and $\kappa_{\max }=1.5$ we observe two phenomena: complete synchronization and the emergence of frequency clusters hierarchical in size, see Figs. 3.2 and 3.3, respectively.

Figure 3.2(a) shows the initial coupling weights, and Fig. 3.2(c) illustrates the spike times of the neurons at the beginning of the simulation. One can observe that while the neurons start with an incoherent spiking, they enhance the coherence already after a few spikes due to the interaction between them as well as the plasticity. The plasticity potentiates the connections of neurons that fire together. A complete synchronization is established and the coupling weights increase to $\kappa_{\max }$ after a transient (Fig. 3.2(b,d)). In a completely synchronized state, the individual neurons spike simultaneously, hence, the spike time differences $\Delta t_{i j}=0$.

The emergence of frequency clusters is shown in Fig. 3.3 for two clusters. The system in Fig. 3.3 possesses the same parameters as in Fig. 3.2, and the difference is just another realization of random initial conditions. In contrast to the synchronized state, the final state shown in Fig. 3.3(f) consist of two groups of synchronized neurons. These cluster states also manifest themselves as two groups of strongly coupled elements in the coupling matrix $\kappa$ (Fig. 3.3(c)). The coupling weights between the neurons from the different groups is very small or zero.

We observe that the largest cluster is formed rather quickly as time evolves, whereas the formation of the small cluster takes much more time. This is illustrated in Fig. 3.4, where the time courses of the mean coupling within each of the two clusters are shown. The average coupling within the big cluster reaches its maximum fast (at $t \approx 1000$, solid curve in Fig. 3.4), whereas the smaller cluster in Fig. 3.3(c,f) is formed through the merging of transient clusters and finally establishes at $t \approx 17000$ (dashed curve in Fig. 3.4).

For the states with more clusters, each new formed cluster is significantly smaller than the previous one, see Fig. 3.5, where three clusters are shown. The spiking period of the cluster appears to be proportional to its size: the bigger the cluster the larger is the period. Simulation of the cases with even more clusters becomes computationally expensive due to large transients. 
(a)
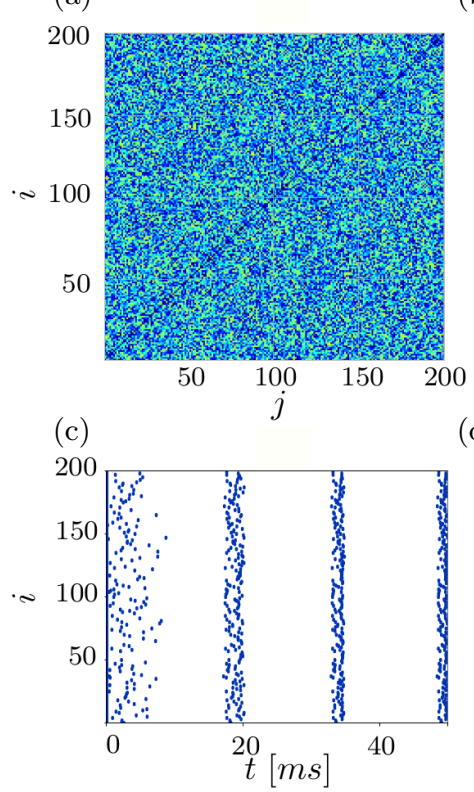

(b)

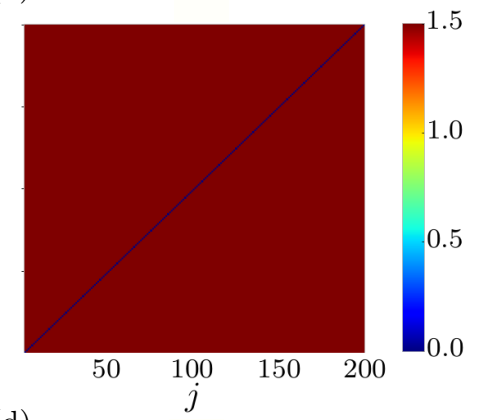

(d)

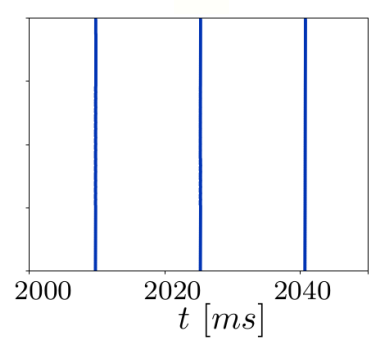

Figure 3.2: Evolution of the coupling matrix $\kappa_{i j}(t)$ starting from random initial conditions and converging to a completely synchronous state. Panel (a) shows initial coupling matrix, (b) the coupling matrix after the transient $t=2000 \mathrm{~ms}$. Raster plot of spiking times at the beginning of simulations (c) and after the transient (d). The asymptotic state $(\mathrm{b}, \mathrm{d})$ is a completely synchronized spiking with all coupling weights $\kappa_{i j}$ potentiated to $k_{\max }$. Other parameters $N=200, \tau_{p}=2, \tau_{d}=5, c_{p}=2, c_{d}=1.6$, and $\kappa_{\max }=1.5$. Figure modified from [ROE19a].

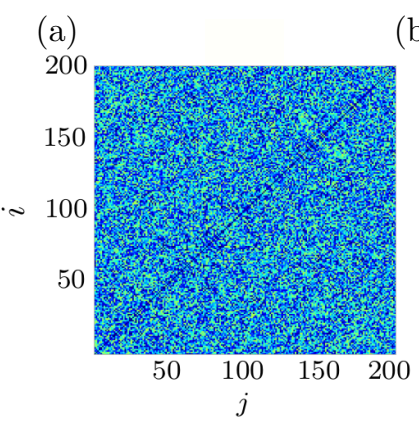

(d)

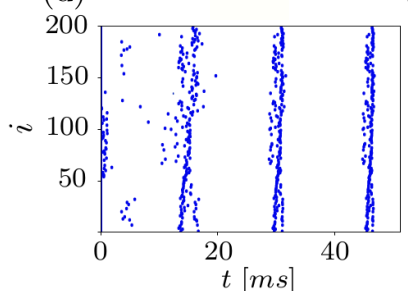

(b)

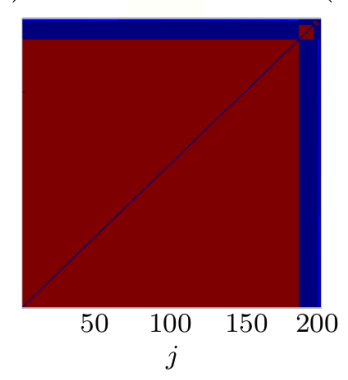

(e)

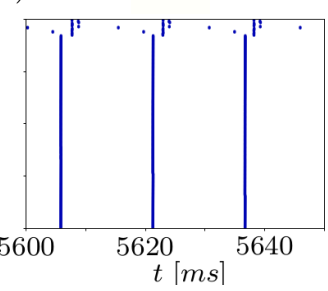

(c)

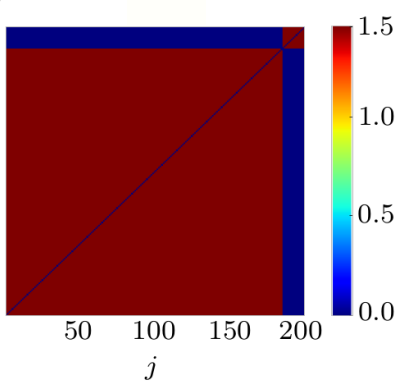

(f)

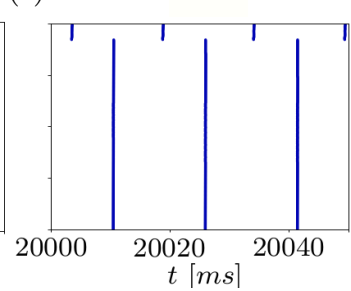

Figure 3.3: Evolution of the coupling matrix $\kappa_{i j}(t)$ starting from random initial conditions and converging to frequency clusters hierarchical in size. Panel (a) shows initial coupling matrix, (b) the coupling matrix after the transient $t=5600 \mathrm{~ms}$, and (c) $t=20000 \mathrm{~ms}$. (d-f) Corresponding raster plots of spike times. The asymptotic state $(\mathrm{c}, \mathrm{f})$ is a hierarchical cluster state with the coupling weights $\kappa_{i j}$ potentiated to $k_{\max }$ within each cluster and small or zero otherwise. Other parameters as in Fig. 3.2. The oscillators are ordered accordingly to their mean frequency. Figure modified from [ROE19a]. 


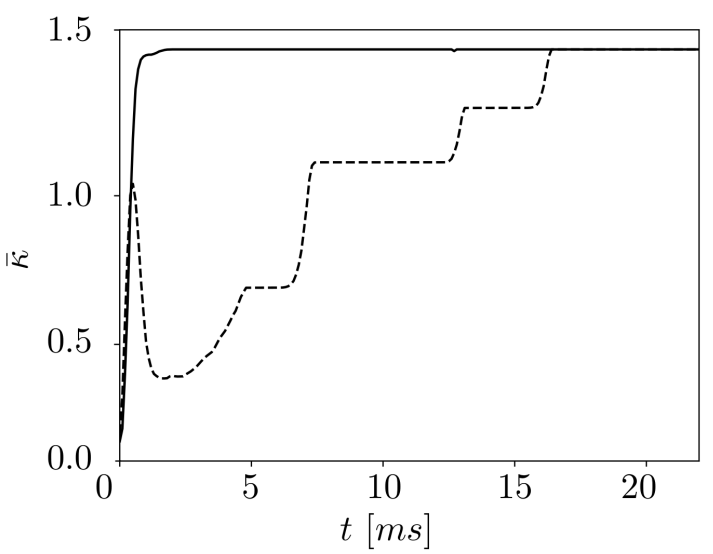

Figure 3.4: Formation of individual clusters over time (corresponds to the dynamical scenario in Fig. 3.3). The dashed and solid curves depict the time course of the mean coupling within the small and big clusters, respectively. Figure taken from [ROE19a].

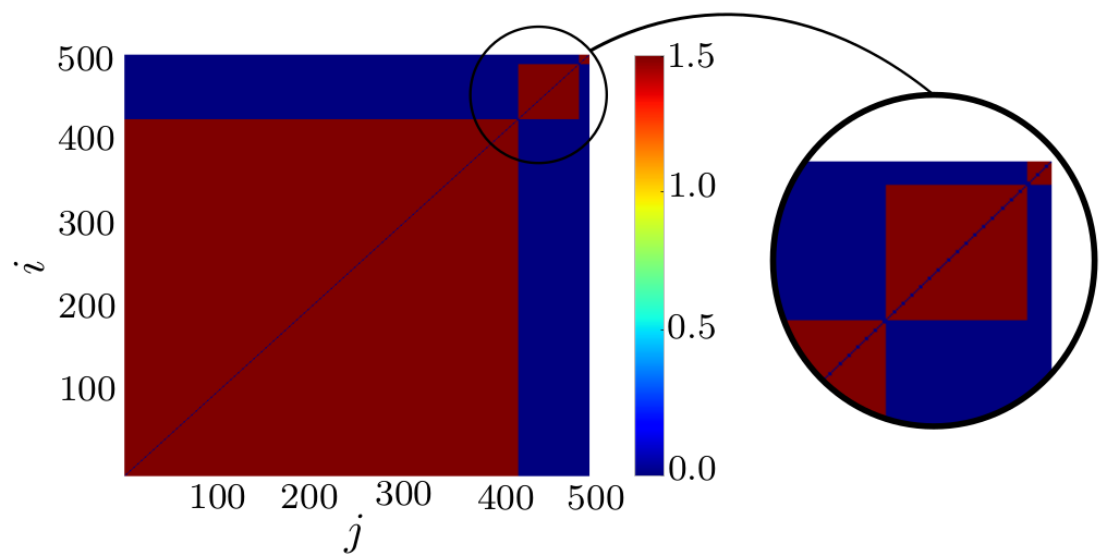

Figure 3.5: Example of a three-cluster state for $N=500, \tau_{p}=2, \tau_{d}=5, c_{p}=2, c_{d}=1.6$, and $\kappa_{\max }=1.5$ with a random initial distribution of $\kappa_{i j}$ in [0,0.75]. Figure taken from [ROE19a].

\section{Clustering with independent random input}

To investigate the robustness of our findings, we added an $\alpha$-train as additional independent random input to the membrane potential $V_{i}$ of every neuron:

$$
\left.I_{i}^{\text {input }}(t)=I\left(V_{r}-V_{i}(t)\right) \sum_{\tau_{i, k}<t} \alpha\left(t-\tau_{i, k}\right) e^{-\alpha\left(t-\tau_{i, k}\right)}\right)
$$

The Eq. (3.4) models a postsynaptic potential (PSP) that is received by the neuron at certain random times $\tau_{i, k}$. The inter-spike interval is Gaussian distributed $\tau_{i, k+1}-\tau_{i, k}=\Delta \tau_{i, k} \sim \mathcal{N}(14 m s, 4 m s)$. $\alpha$ is set to $24 /\left\langle\Delta \tau_{i, k}\right\rangle$.

The numerical simulations Fig. 3.6 show that the clustering is still observed under the influence of random input $I_{i}^{\text {input }}(t)$ of intensity $I$. More specifically, for sufficiently weak perturbations with $I<0.01$, all three clusters survive (Fig. 3.6(a)). With increasing the amplitude $I$, the clusters start to decouple. The smaller clusters are affected first (Fig. 3.6(b-d)), they start desynchronizing at $I=0.01$. The biggest cluster keeps shrinking in size while $I$ is increased and finally for $I=0.07$ the whole network decouples (Fig. 3.6(e)). 


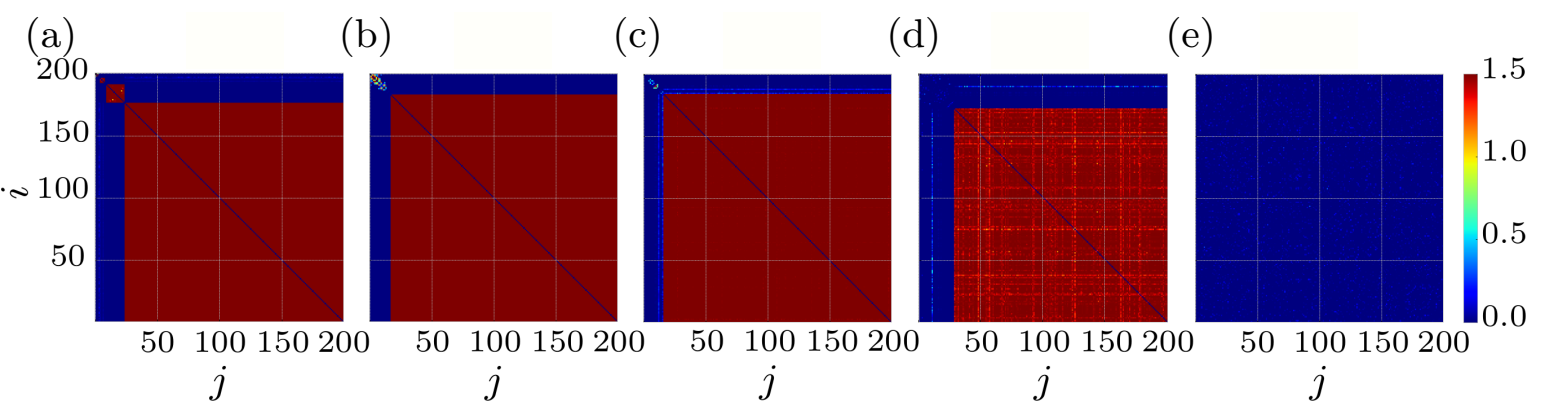

Figure 3.6: Coupling matrices for $t=10000 \mathrm{~ms}$ and different amplitudes of independent random input $I$ (see Eq. (3.4)). (a) $I=0.005$, (b) $I=0.01$, (c) $I=0.02$, (d) $I=0.05$ and (e) $\mathrm{I}=0.07$. All other parameters as in Fig. 3.5. Figure modified from [ROE19a].

\subsection{Emergence of two-cluster states}

In this section we numerically show that depending on the relative size of the two clusters, such two-cluster states can be either dynamically stable or transient leading to complete synchronization. In order to investigate the cluster stability, we initialize the system in a two cluster state with the number of neurons $N_{s}$ in the small cluster and $N_{b}=N-N_{s}$ in the big cluster. The total number of neurons is set to $N=50$. The inter-cluster couplings are set to zero initially while the intra-cluster couplings equals $\kappa_{\max }$. All neurons in the same cluster are initialized with the same initial conditions, so the clusters are fully synchronized at $t=0$.

Figure 3.7(a) shows frequency difference of two uncoupled clusters as a function of the size of the small cluster. The frequency difference demonstrates an almost linear dependence on the cluster size and decays as the size of the smaller cluster increases. Moreover, we also observe that clusters with sufficiently different sizes are stable while the clusters of similar sizes, in the considered case with $N_{s}>8$, are transient, merge into a single cluster and eventually lead to a stable completely synchronous state, see Fig. 3.8.

Although the threshold of how different the clusters should be in order to be stable is certainly model dependent, the synchronization of similar clusters is a general property. The merging of two clusters can be explained qualitatively as follows. Initially uncoupled clusters evolve
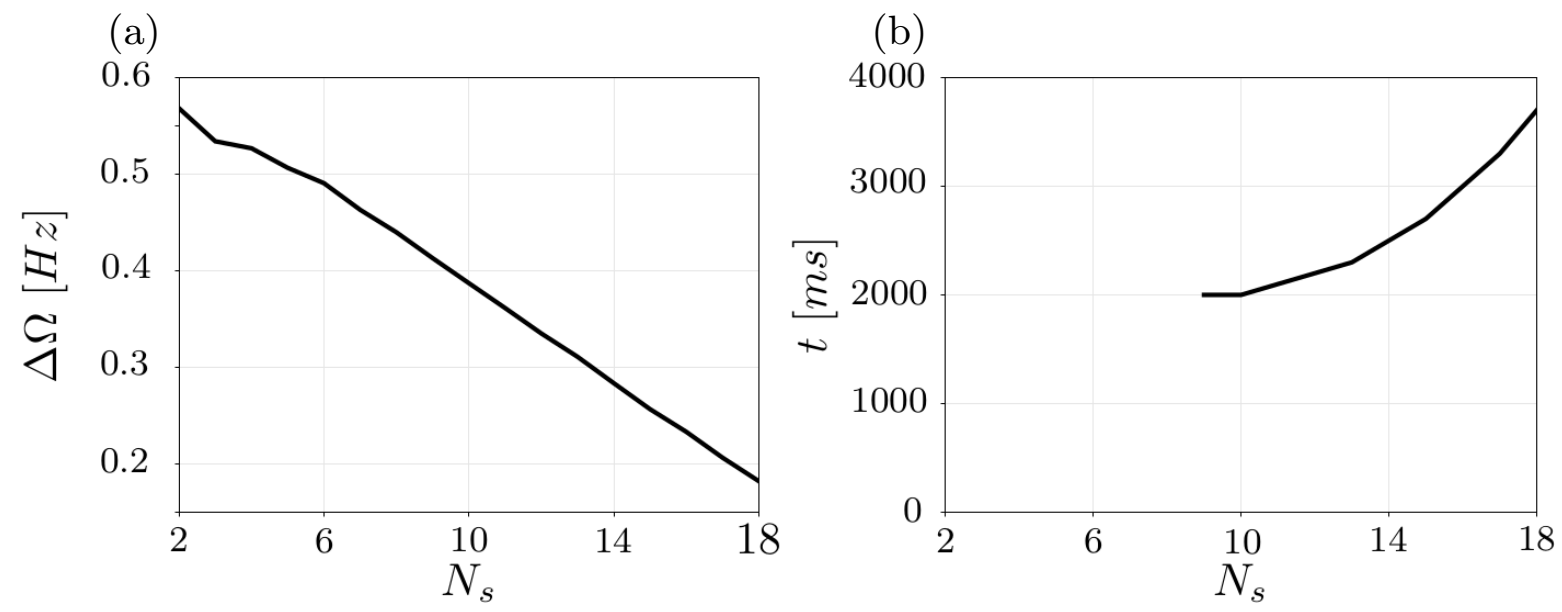

Figure 3.7: (a) Difference between synchronization frequencies of the two clusters for different size of the smaller cluster $N_{s}$. (b) Time until cluster fusion for different initial size of the smaller cluster $N_{s}$. Figure modified from [ROE19a]. 
each with their natural frequencies $\Omega_{s}$ and $\Omega_{b}$. If their sizes are different, then $\Omega_{s} \neq \Omega_{b}$, and the clusters arrive in-phase periodically with the "beating" frequency $\Delta \Omega=\Omega_{b}-\Omega_{s}$. As soon as such an in-phase episode occurs, the interspike intervals $\Delta t_{i j}$ between any two neurons from different clusters become small and, hence, due to the plasticity rule $W$ (see Eq. (3.2) and Fig. 3.1) the inter-cluster coupling weights increase. Moreover, the duration of such an in-phase episode depends on the frequency difference between the clusters. As a result, for a small frequency difference $\Delta \Omega$, the time interval where the clusters are practically in-phase is sufficiently long in order to potentiate the coupling weights to their maximum value. This unites the two clusters into one. In contrast, for large $\Delta \Omega$, such an episode is short, and the inter-cluster coupling remains small, which keeps the clusters oscillating at different frequencies in a stable manner.

Figure 3.8(a-c) displays an example of two-cluster stable state with $N_{s}=8$. Starting from the two-cluster state, after $t=1500 \mathrm{~ms}$, the coupling between the clusters increases, see Fig. 3.8(b) due to the "in-phase episode" when the clusters are synchronous. Afterwards, however, the inter-cluster coupling weights return to their initial configuration (Fig. 3.8(c)), since the spike time differences for neurons from different clusters are again far enough apart to cause the depression of the inter-cluster synapses. Such a process is repeated every time the clusters meet and is typical for the stable cluster states. A typical case of transient clusters is presented in Fig. 3.8(d-f) for $N_{s}=9$. The inter-cluster coupling is again potentiated when the clusters meet, but it does not decrease again, and the clusters merge in a single cluster of a fully coupled and synchronized regime (Fig. 3.8(f)). The transient time that could be elapsed until the cluster fusion depends on the cluster size as illustrated in Fig. 3.7(b).

Figures 3.8(g,h) show how the spiking frequency of the clusters change over time. During the in-phase episode, the cluster with the higher natural spiking rate slows down significantly, while the slower cluster (with larger number of neurons $N_{b}$ ) speeds up a little. For a stable cluster state the cluster frequencies again deviate from each other (Fig. 3.8(g)), whereas all neurons fire with the same frequency when the clusters unite into one (Fig. 3.8(h)). We found this phenomenon for different numbers of neurons and different $\kappa_{\max }$. Increasing $\kappa_{\max }$ increases the initial period difference, but the behavior in general stays the same.

Figure 3.9 shows the dynamics of the mean synaptic activity $S(t)=\frac{1}{N} \sum_{i=1}^{N} s_{i}(t)$ of the network in the case of two stable clusters, which models the dynamics of LFP. During the in-phase episodes of the two clusters, $S(t)$ has a higher amplitude, because both clusters spike synchronously. The maximum amplitude is generated by maximum synchronization in the network. The low amplitude of $S(t)$, on the other hand, corresponds to the time intervals when the clusters are out of phase. In the latter case, the mean synaptic activity shows two peaks, the higher peak is generated by the larger cluster and the lower by the smaller one, see Fig. 3.9(b). For the considered case, the synchronized oscillations of individual neurons in the clusters take place at a time scale of several milliseconds (period $~ 15$ ms, Fig. 3.9(b)), see also Fig. 3.8(g,h). The neurons are tonically spiking. The frequency difference $\Delta \Omega$ between clusters is, however, of the order of sub-Hz, because the corresponding cluster frequencies are close to each other (Fig. 3.8(g,h)). Then the modulation of $S(t)$ is observed at a much slower timescale of a few seconds, which is of two orders of magnitude slower than the intrinsic neural firing, see Fig. 3.9(a), as observed in empirical data of the brain activity [MAN07, MAG12a].

In the following section, a phenomenological model is introduced in order to further investigate the dynamics of two clusters. 
(a) $t=0$

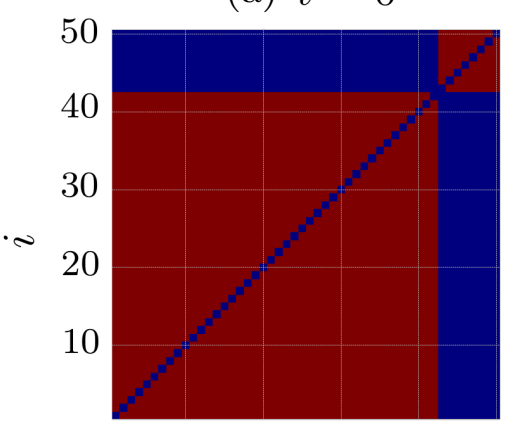

(d) $t=0$

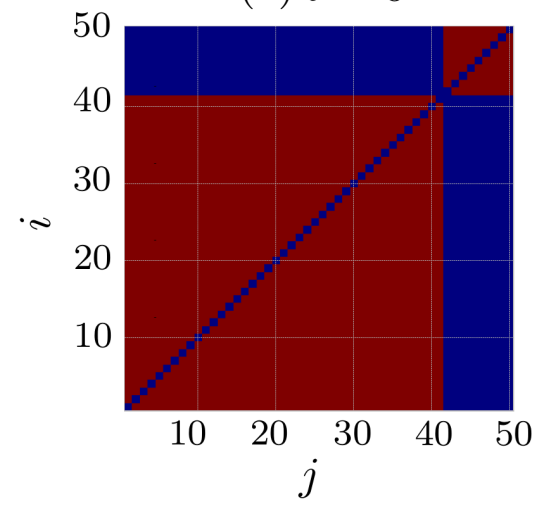

(g)

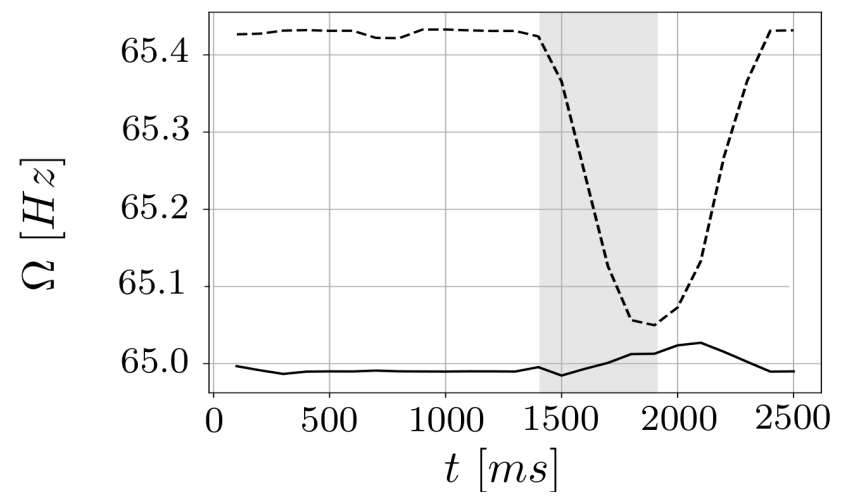

(b) $t=1600$

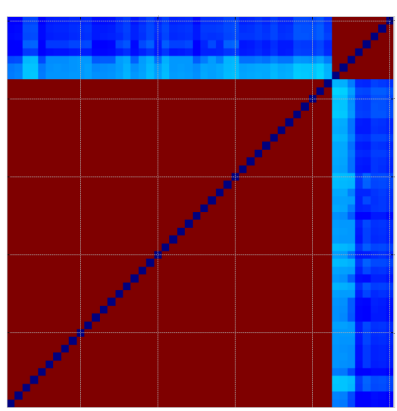

(e) $t=1600$

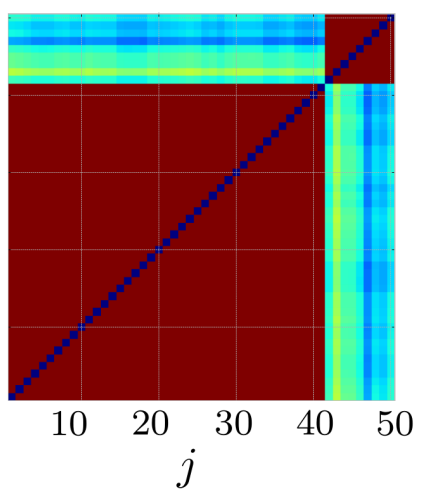

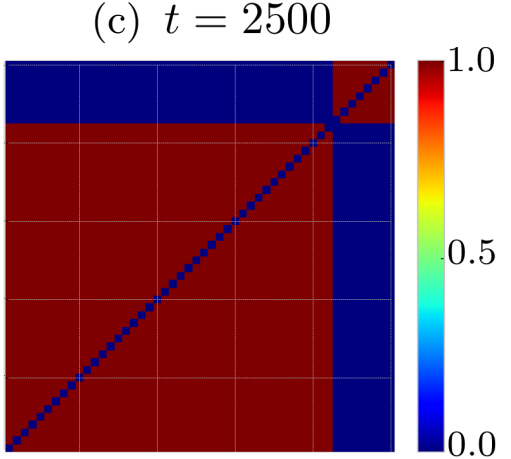

(f) $t=2500$

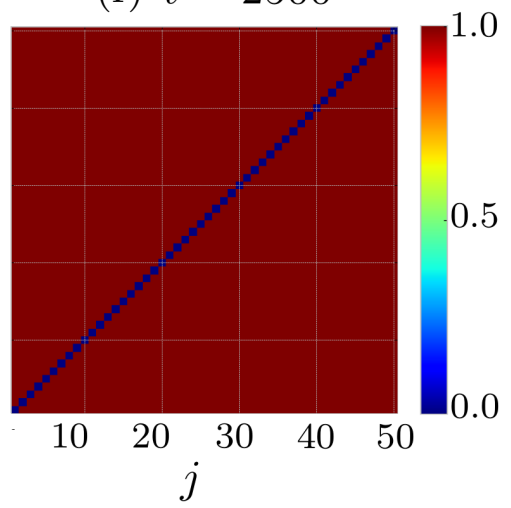

(h)

Figure 3.8: Evolution of the coupling matrix for $N=50$ and the number of neurons $N_{S}=8$ (a-c) and $N_{S}=9(\mathrm{~d}-\mathrm{f})$ in the small cluster. In panels (a-c) the clusters are stable, while in (d-f) they are merging to one synchronous cluster. (g, h) Time courses of the spiking synchronization frequencies of small ( $N_{s}$ neurons) and large ( $N_{b}$ neurons) clusters depicted by dashed and solid curves, respectively, for (g) $N_{s}=8$ and $N_{b}=42$ and (h) $N_{s}=9$ and $N_{b}=41$. Parameter $\kappa_{\max }=1.0$. Figure modified from [ROE19a]. 
(a)

(b)

Zoom of (a)
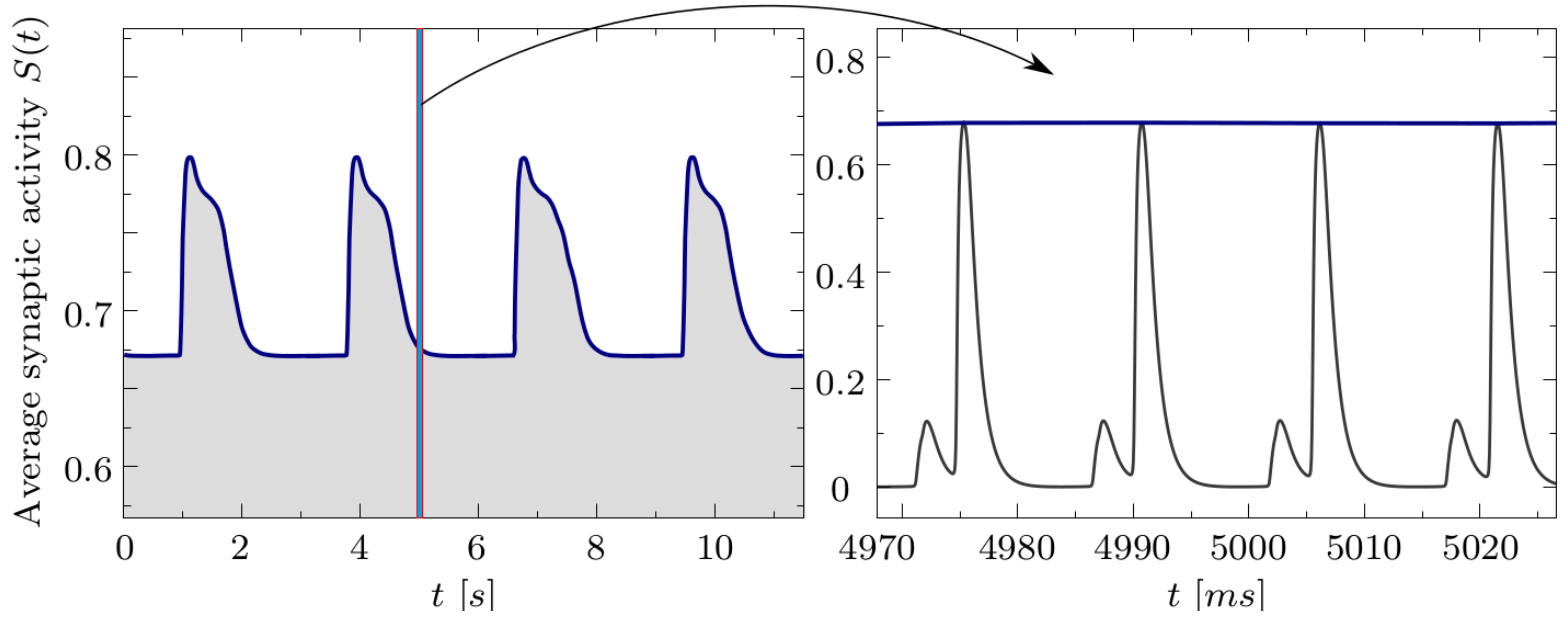

Figure 3.9: Mean synaptic activity $S(t)$ of the neural population in the case of stable two cluster state. Panel (a) shows the dynamics of $S(t)$ on the time interval of $12 \mathrm{~s}$, where modulation of the amplitude (blue line) is visible, while the fast oscillations are not recognized on this timescale. The maximum amplitude corresponds to the two clusters being synchronised, while the low amplitude corresponds to the clusters being out of phase. Panel (b) shows the zoom of a small time interval. The modulation takes place on the timescale which is two orders of magnitude larger than the individual spikes of $S(t)$ as well as individual neural spikes in both clusters. Cluster frequencies $\omega_{1}=0.065012 \mathrm{kHz}$ and $\omega_{2}=0.065416 \mathrm{kHz}$. The corresponding period of modulation is $T \approx 2.5 \mathrm{~s}$. Figure modified from [ROE19a]. 


\subsection{Phenomenological model with phase difference-dependent plasticity}

In this section we introduce a reduced qualitative model for the coupling and phase difference of two clusters. The model is based on the assumption that oscillators are synchronized identically within each cluster and the coupling between the clusters is weak. As a result, the interaction between oscillatory clusters can be described in the framework of two coupled phase oscillators that are interacting via their phase differences [HOP97, PIK01, GUC75, WIN01]

$$
\begin{aligned}
& \dot{\varphi}_{1}=\omega_{1}-F_{1}\left(\varphi_{1}-\varphi_{2}\right), \\
& \dot{\varphi}_{2}=\omega_{2}-F_{2}\left(\varphi_{2}-\varphi_{1}\right),
\end{aligned}
$$

where $\omega_{1}$ and $\omega_{2}$ are the natural frequencies of the individual clusters, $F_{1}$ and $F_{2}$ are effective interaction functions. For the phase difference $\varphi=\varphi_{1}-\varphi_{2}$, this system reads

$$
\dot{\varphi}=\omega-F(\varphi)
$$

where $\omega=\omega_{1}-\omega_{2}$ is the difference of the natural frequencies, and $F(\varphi)=F_{1}(\varphi)-F_{2}(-\varphi)$.

Since the clusters are synchronized for a sufficiently small frequency mismatch $\omega$, the periodic interaction function $F(\varphi)$ must satisfy $F(0)=0$ and $F^{\prime}(0)>0$. The latter means that there is a stable equilibrium $\varphi=0$ for small $\omega$. Aiming at a qualitative insight, we further simplify the model by assuming that $F(\varphi)=\sigma \sin (\varphi+\alpha)$, where $\sin \varphi$ can be viewed as a first Fourier harmonic of the interaction function and $\sigma$ as an effective coupling weight. The parameter $\alpha=\sin ^{-1}\left(\omega / \sigma_{\max }\right)$ is a constant phase shift assuring that the phase difference of the synchronized cluster is zero. In fact, for small $\omega$, this parameter is also small and it does not play important role in the qualitative behavior of the model apart from a small shift of the synchronized state to $\varphi=0$.

Another component of the model is the plasticity-driven changes of the coupling $\sigma$. In order to derive the equation for $\sigma$, we consider the STDP update in the case of a periodic motion of the clusters. We assume that the coupling $\sigma$ is proportional to an averaged coupling between the clusters. This is a natural assumption in the case of weakly coupled systems. Let us find out how the update of the intercluster coupling depends on the phase difference $\varphi$. For a given phase difference $\varphi$ and the frequencies $\omega_{1}=\bar{\omega}+\omega / 2, \omega_{2}=\bar{\omega}-\omega / 2$ (here we introduced the mean frequency $\bar{\omega}$ ), the spiking period of the both clusters can be approximated as $T \approx 2 \pi / \bar{\omega}$ up to small terms of order $\omega$, and the distance $\Delta T$ between the spikes of two clusters

$$
\Delta T=\left[T \frac{\varphi_{1}}{2 \pi}-T \frac{\varphi_{2}}{2 \pi}\right] \bmod T=\left[T \frac{\varphi}{2 \pi}\right] \bmod T \approx \frac{\varphi \bmod 2 \pi}{\bar{\omega}} .
$$

Since the spike time differences $\Delta T$ and $T-\Delta T$ occur recursively, see Fig. 3.10(b), the updates per unit time sum to the function

$$
\frac{\delta}{T}(W(T-\Delta T)+W(\Delta T))=\frac{\delta \bar{\omega}}{2 \pi} G(\varphi),
$$


(a)

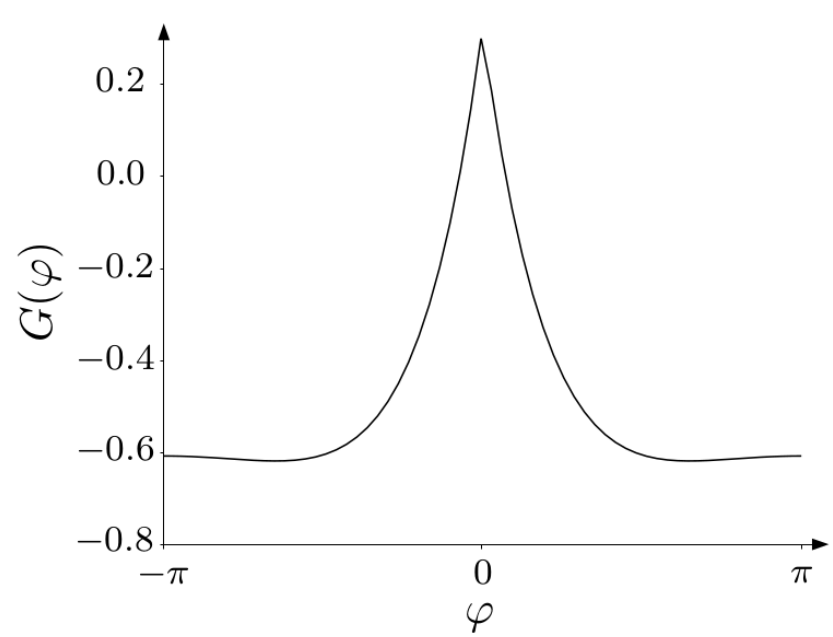

(b)

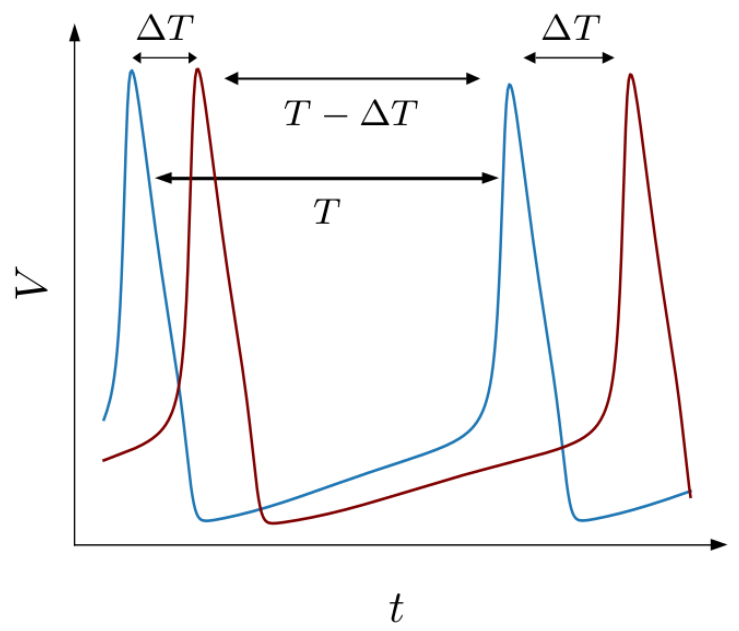

Figure 3.10: (a) Update function $G(\varphi)$ for $\tau_{p}=2, \tau_{d}=5, c_{p}=2$, and $c_{d}=1$.6. (b) Schematic spiking of two oscillators with spike time difference $\Delta T$ and periods close to $T$. Figure modified from [ROE19a].

where

$$
G(\varphi):=W\left(\frac{2 \pi-(\varphi \bmod 2 \pi)}{\bar{\omega}}\right)+W\left(\frac{\varphi \bmod 2 \pi}{\bar{\omega}}\right)
$$

Since the update of $\sigma$ is proportional to the obtained function, and taking into account the smallness of $\delta$, this update can be written as $\dot{\sigma}=\varepsilon G(\varphi)$, where $\varepsilon$ is a small parameter of the coupling adaptation that controls the scale separation between the fast dynamics of the clusters and the slow dynamics of the coupling.

Additionally, the coupling strength $\sigma(t)$ should be bounded to the interval $\left[0, \sigma_{\max }\right]$ by imposing cut-off conditions. More specifically, the derivative $\dot{\sigma}$ is discontinuous at the boundaries $\sigma=0$ and $\sigma=\sigma_{\max }$, i.e. $\dot{\sigma}=\max \{0, \varepsilon G(\varphi)\}$ for $\sigma=0$ and $\dot{\sigma}=\min \{0, \varepsilon G(\varphi)\}$ for $\sigma=\sigma_{\max }$. The considered cut-off corresponds to "hard" bound conditions [SON00]. Another possibility would be "soft" or "multiplicative" bounds [RUB01], when the update is proportional to the distance to the boundary. We consider here the hard bound, since it corresponds to the hard bound of the STDP rule for Hodgkin-Huxley system.

The final phenomenological model reads as follows

$$
\begin{aligned}
& \dot{\varphi}=\omega-\sigma \sin (\varphi+\alpha), \\
& \dot{\sigma}=\varepsilon \cdot \begin{cases}G(\varphi) & \text { for } 0<\sigma<\sigma_{\max } \\
\max \{0, G(\varphi)\} & \text { for } \sigma=0, \\
\min \{0, G(\varphi)\} & \text { for } \sigma=\sigma_{\max } .\end{cases}
\end{aligned}
$$

with frequency mismatch $\omega>0$ and $\alpha=\sin ^{-1}\left(\omega / \sigma_{\max }\right)$. 

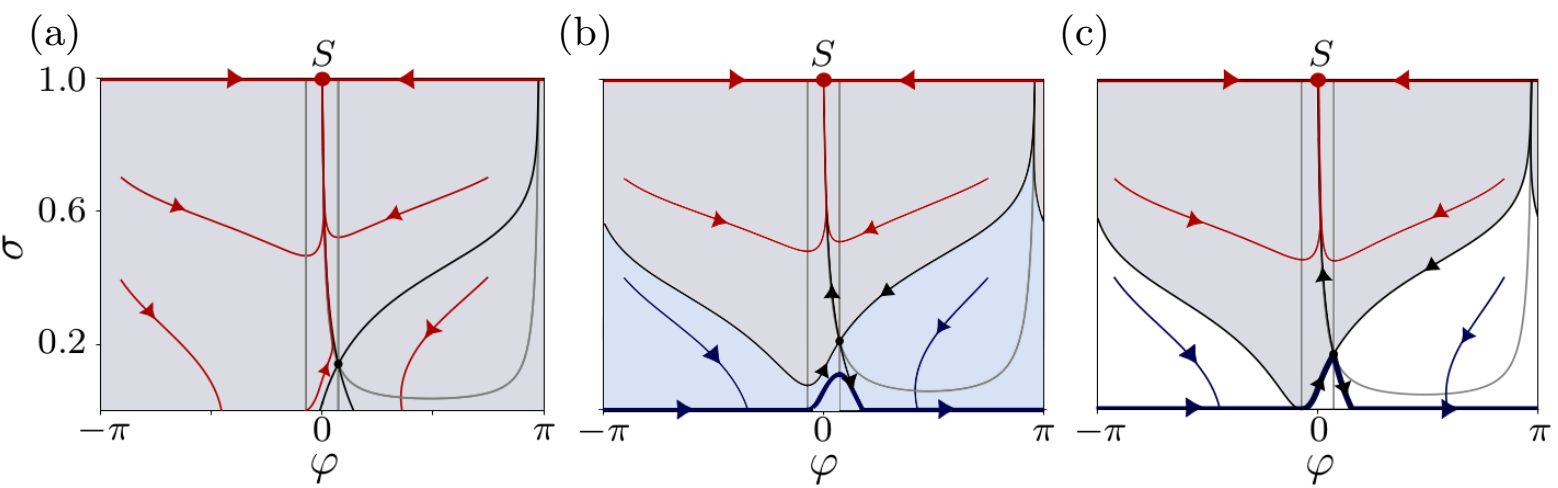

Figure 3.11: Phase portraits of model (3.10)-(3.11) for (a) monostable regime of complete synchronization; (b) coexistence of stable synchronized and clustered states; and (c) bifurcation moment of transition between the phase portraits illustrated in (a) and (b). The basins of attraction of the synchronized regime (point $S$ ), clustered state (limit cycle indicated by thick black curve) and the saddle fixed point $\left(\varphi^{*}, \sigma^{*}\right)$ are depicted by gray, blue, and white colors, respectively. The nullclines of the system and stable and unstable manifolds of the saddle point are indicted by the thin gray and black curves, respectively. Parameters (a) $\omega=0.037 \mathrm{kHz}$, (b) $\omega=0.06 \mathrm{kHz}$, (c) $\omega \approx 0.455 \mathrm{~Hz}$, and the other parameters $\tau_{p}=2, \tau_{d}=5, c_{p}=2, c_{d}=1.6$, and $\varepsilon=0.08$. Figure modified from [ROE19a].

\subsubsection{Properties of the model}

Phase space of system (3.10)-(3.11) is two dimensional with $(\varphi, \sigma) \in S^{1} \times\left[0, \sigma_{\max }\right]$. The nullclines are given by $G(\varphi)=0$ for $\dot{\sigma}=0$ and $\sigma=\omega / \sin (\varphi+\alpha)$ for $\dot{\varphi}=0$ in the internal points of the phase space. For the parameter values as in Fig. 3.11, the $\varphi$-nullcline corresponds to the two lines $\varphi=\varphi^{*} \approx 0.23$ and $\varphi=-\varphi^{*}$, while the $\sigma$-nullcline to a U-shaped nonlinear curve (grey lines in Fig. 3.11).

There is just one fixed point $\left(\varphi^{*}, \sigma^{*}=\omega / \sin \varphi^{*}\right)$ of saddle-type within the region $\sigma \in\left(0, \sigma_{\max }\right)$. This point is given by the intersection of the nullclines. Figure 3.11 shows this fixed point and its stable and unstable separatrixes (black lines). An additional fixed point as well as periodic attractor emerge in system (3.10)-(3.11) due to the non-smoothness at the boundaries. More specifically, three situations are observed:

(I): One globally stable fixed point $S=\left(0, \sigma_{\max }\right)$ which corresponds to the fusion of the two clusters into one. The coupling $\sigma=\sigma_{\max }$ and the phase difference is zero at the fixed point, see Fig. 3.11. All orbits are approaching this stable fixed point with time. This corresponding phase portrait is shown in Fig. 3.11(a).

(II): Coexistence of the stable fixed point $S=\left(0, \sigma_{\max }\right)$ and a stable periodic orbit, see Fig. 3.11(b). As in the case (I), the fixed point corresponds to the merging of two clusters. The periodic orbit corresponds to two simultaneously existing clusters. The clusters possess different frequencies and, as a result, the phase difference is not bounded and rotate along the circular direction $\varphi$. Part of the periodic orbit is located on the boundary $\sigma=0$, i.e. vanishing inter-cluster coupling. The coupling $\sigma(t)$ increases between $-\varphi^{*}$ and $\varphi^{*}$ and decreases otherwise. In fact, one can parameterise the coupling $\sigma$ by the phase $\varphi$ on the periodic attractor. In the case when $\sigma\left(\varphi^{*}\right)<\sigma^{*}$, the solution returns to the boundary $\sigma=0$, moves along it till the orbit reaches the point $\left(-\varphi^{*}, 0\right)$, and the periodic motion repeats.

(III): When $\max _{\varphi} G(\varphi)<0$ then there exists globally stable periodic solution $\varphi=\omega t+\varphi_{0}$, $\sigma=0$. In such a case, the fixed point on the boundary disappears. Formally, this corresponds 
to an uncoupling between the clusters. However, in the original Hodgkin-Huxley system, this parameter regime corresponds to complete uncoupling of all oscillators because of the depression of all synapses.

In fact, the parameter boundary between the cases (I) and (II) is determined by the condition $\sigma\left(\varphi^{*}\right)=\sigma^{*}$, which can be interpreted geometrically as hitting the point $\left(-\varphi^{*}, 0\right)$ by the stable manifold of the saddle equilibrium point, see Fig. 3.11(c). In this special case, the saddle equilibrium attracts the whole set of points from the phase space that is below the stable manifolds, see white area in Fig. 3.11(c). In case (II), the separation between the basins of attraction of the fixed point and the periodic orbit are given by the saddle equilibrium and its stable manifolds. A sufficient condition for the case (III) is given by $c_{d} \geq c_{p}$ and $\tau_{d} \geq \tau_{p}$. Under these conditions $G(\varphi) \leq 0$ for all $\varphi$.

Summarizing, the case (II) corresponds to the situation when clusters are stable and do not merge into one. For this, initial conditions must belong to the basin of attraction of the periodic solution (Fig. 3.11(b), blue domain). The analysis of the phenomenological model indicates that the cluster case always coexists with stable complete synchronization.

\subsubsection{Comparison of the model and cluster dynamics in Hodgkin-Huxley network}

In order to compare dynamics of the phenomenological model (3.10)-(3.11) and the original system (3.1)-(3.2), we ran a series of simulations of the Hodgkin-Huxley network for parameter values that allow for a stable two-cluster solution. The phases of the clusters are calculated

as $\varphi_{1,2}(t)=2 \pi \frac{t-t_{k}}{t_{k+1}-t_{k}}+2 \pi k$ for $t \in\left[t_{k}, t_{k+1}\right)$, where $\left\{t_{1}, \ldots, t_{n}, \ldots\right\}$ are spiking times with $t_{k}<t_{k+1}$ [PIK01]. Correspondingly, the phase difference is $\varphi_{H}(t)=\varphi_{1}(t)-\varphi_{2}(t)$. The coupling measure $\sigma_{H H}$ is given by the mean inter-cluster coupling.

Extracting the quantities $\sigma_{H H}$ and $\varphi_{H H}$ from the numerically computed solutions of HodgkinHuxley system (3.1)-(3.3) we obtain a two-dimensional projection of the solution to the plane $\left(\varphi_{H H}, \sigma_{H H}\right)$, see Fig. 3.12. The discontinuities in the orbits are related to the discrete STDP updates. Additionally, since the phases $\varphi_{1,2}(t)$ can be firstly accessed after the both clusters fired, some of the area of the phase diagram (see white area in Fig. 3.12) was not accessible. This "empty" area corresponds to anti-phase initial conditions, which are very sensitive, and, after each cluster fires, they appear immediately either in the red or blue area. Nevertheless, the behavior has the same qualitative features as in the phenomenological model, compare Figs. 3.11 and 3.12.

\subsubsection{Criteria for the emergence of frequency clusters}

Model (3.10)-(3.11) can be used to describe plasticity functions, which lead to multiple clusters. For this, we investigate numerically the condition $\sigma\left(\varphi^{*}\right)=\sigma^{*}$. More specifically, system (3.10)(3.11) was initialized at the point $\left(-\varphi^{*}, 0\right)$ and numerically integrated forward in time. If $\sigma\left(\varphi^{*}\right)<$ $\sigma^{*}$, the two clusters are stable and do not merge. This procedure can be repeated for different parameter values.

In order to restrict the set of plasticity parameters, we fix $\tau_{p}=2$ and $\tau_{d}=5$ and vary $c_{p}$ and $c_{d}$. The results of the simulation are shown in Fig. 3.13(a). The white, black and grey parameter 


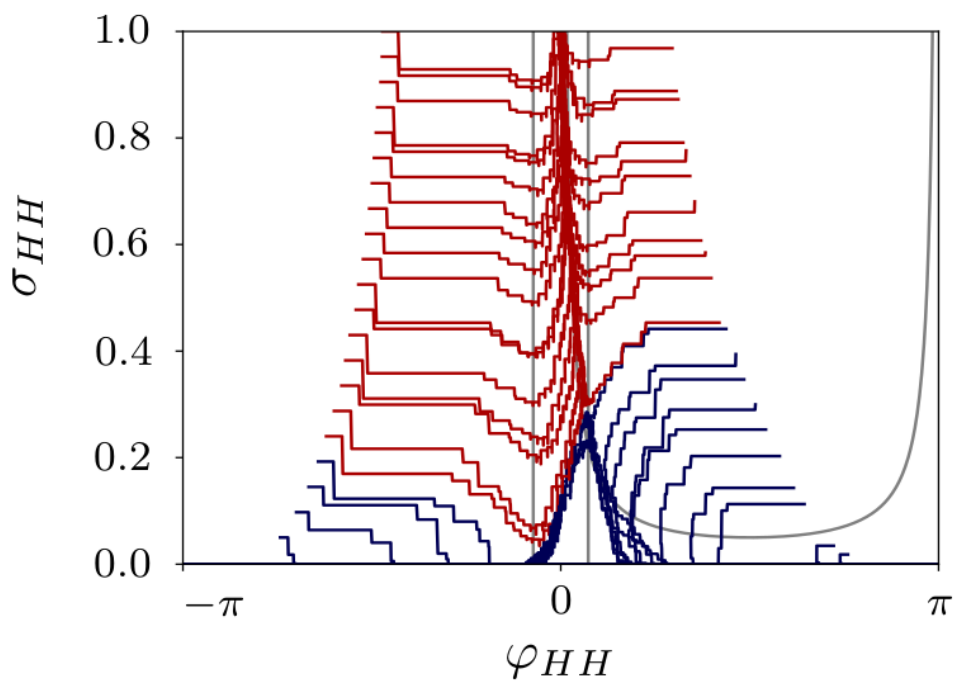

Figure 3.12: Dynamics of the phase difference between the clusters $\varphi_{H H}$ and mean inter-cluster coupling $\sigma_{H H}$ for the solutions of the Hodgkin-Huxley system (3.1)-(3.3) for different initial conditions. $N=50$ with $N_{s}=7$ neurons in the small cluster and $N_{b}=43$ in the big one. Red orbits converge to the regime of complete synchronization, and blue trajectories lead to a stable two-cluster solutions. The nullclines of the phenomenological model are shown in gray. Other parameters: $\tau_{p}=2, \tau_{d}=5, c_{p}=2, c_{d}=1.6$, and $\kappa_{\max }=1.5$. Figure taken from [ROE19a].

areas correspond to the appearance of stable periodic solution of (3.10)-(3.11) (case (II)), globally stable fixed point (case (I)) and the case (III), respectively.

In order to compare the parameter regions obtained for the phenomenological model (Fig. 3.13(a)) with those for the original Hodgkin-Huxley system, we ran numerical simulations of system (3.1)-(3.3) with $N=50$ neurons and $N_{s}=7$ neurons in the small cluster. Starting from the twocluster state, we monitor the dynamics of the clusters. Figure 3.13(b) shows the results: the white region corresponds to the case when the clusters survive and stay apart after the simulation time 3000 ms, black - when the clusters merge into one synchronous group, and grey - when the clusters split into uncoupled neurons. This behaviour stays qualitatively the same for different cluster sizes. However, depending on the frequency difference between the clusters, the set of parameters allowing stable cluster states may change its size.

Comparison of the results for the phenomenological system and the Hodgkin-Huxley system in the Figs. 3.13 $(\mathrm{a}, \mathrm{b})$ shows that the phenomenological model provides a reasonable approximation. 
(a)

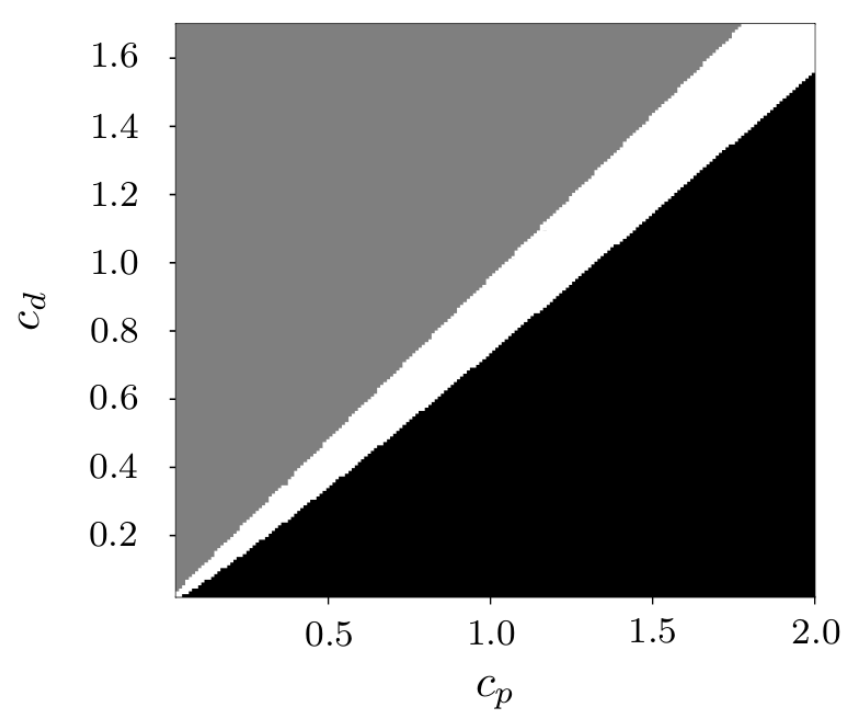

(b)

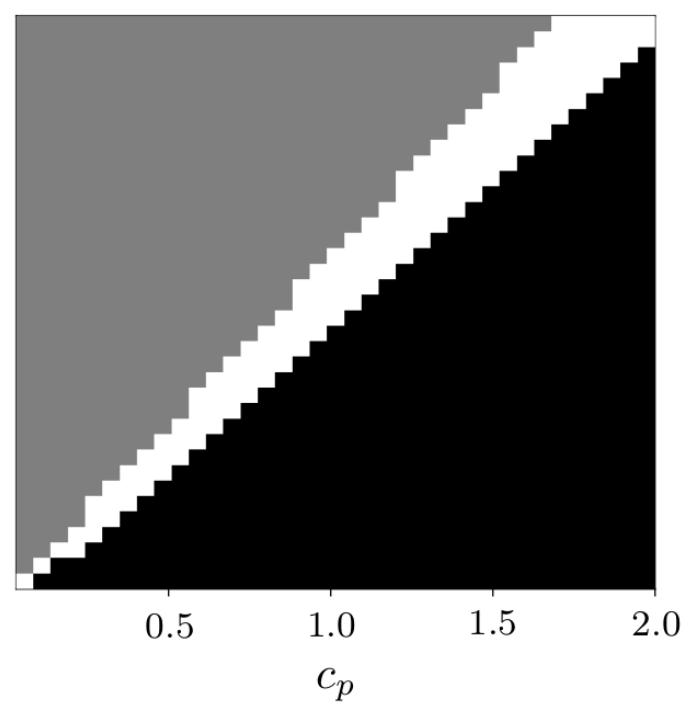

Figure 3.13: Panel (a): system (3.10)-(3.11). White region: stable periodic solution coexisting with a stable fixed point, case II. Black region: globally stable fixed point, case I. Grey region: globally stable periodic solution with $\sigma=0$. Panel (b): original system (3.1)-(3.3). White: stable two-clusters (white); black: stable synchrony and no stable clusters; grey: decoupling of all neurons. Other parameters $\tau_{p}=2, \tau_{d}=5, N=50, N_{s}=7$, and $\kappa_{\max }=1$. Figure modified from [ROE19a].

\subsection{Summary}

In summary, our results show that adaptive neural networks are able to generate self-consistently dynamics with different frequency bands. In our case, each cluster corresponds to a strongly connected component with a fixed frequency. Due to a sufficiently large difference of the cluster sizes and frequencies, the inter-cluster interactions are depreciated, while the intracluster interactions are potentiated. In this study, we have described the mechanisms behind the formation and stabilization of these clusters. In particular, we have explained why the significant difference between the cluster sizes is important for the decoupling of the clusters. From a larger perspective, the decoupling of the clusters in our case is analogous to the decoupling of timescales in systems with multiple timescales.

In addition, we have presented a two-dimensional phenomenological model which allows for a detailed study of the clustering mechanisms. Despite of the approximations made by the derivation, the model coincides surprisingly well with the adaptive Hodgkin-Huxley network. Using the phenomenological model, we have found parameter regions of the plasticity function, where stable frequency clustering can be observed.

Clustering behavior also emerges at the brain scale, where synchronized communities of brain regions constituting large distributed functional networks can intermittently be formed and dissolved [DEC09, PON15]. Such clustering dynamics can shape the structured spontaneous brain activity at rest as measured by fMRI. In this study, we have shown that slow oscillations based on the modulation of synchronized neural activity can already be formed at the resolution level of a single neural population if adaptive synapses are taken into account. These modulations of the amplitude of the mean field can be generated in a stable manner, see Fig.3.9 and Ref. [POP15]. 
The mechanism relies on fluctuations of the extent of synchronization of tonically firing neurons. This is caused by the splitting of the neural population into clusters and the corresponding cluster dynamics. It might contribute to the emergence of slow brain rhythms of electrical (LFP, EEG) and metabolic (BOLD) brain activity reported by Refs. [MAN07, MAG12a, MON08, ALV14].

However, other mechanisms for generating slow oscillations are possible. The papers [BAZ02, COM03] discussed the emergence of slow oscillatory activity $(<1 \mathrm{~Hz})$ that can be observed in vivo in the cortex during slow-wave sleep, under anesthesia or in vitro in neural populations. The suggested mechanism relies on the corresponding modulation of the firing of individual neurons, and the slow oscillation at the population level was proposed to be the result of very slow bursting of individual neurons that synchronize across the neural population. In contrast, the present work shows that the slow oscillations of the population mean field can also emerge when the firing of individual neurons is not affected. The neurons may tonically fire at high frequencies. The amplitude of the population mean field then oscillates at much lower frequencies due to the slow modulation caused by the cluster dynamics. Additionally, more recent work suggested that slow collective oscillations in neural networks with spike timing-dependent plasticity are induced by the so-called Sisyphus effect unveiled using a free energy approach on the temporal behavior of the order parameter. 


\section{One-cluster states in adaptive networks of coupled phase oscillators}

The findings in Chapter 3 suggested that the emergence of frequency clusters in neuronal populations with synaptic plasticity is well described by a phase oscillator model with phase difference-dependent plasticity. A model of this kind was introduced in Ref. [AOK09, KAS17], see also (2.25)-(2.26) in Section 2.3.3, and reads

$$
\begin{aligned}
\frac{d \phi_{i}}{d t} & =\omega-\frac{1}{N} \sum_{j=1}^{N} \kappa_{i j} \sin \left(\phi_{i}-\phi_{j}+\alpha\right), \\
\frac{d \kappa_{i j}}{d t} & =-\epsilon\left(\kappa_{i j}+\sin \left(\phi_{i}-\phi_{j}+\beta\right)\right) .
\end{aligned}
$$

System (4.1)-(4.2) has attracted a lot of attention recently [SEL02, REN07, AOK09, AOK11, PIC11a, TIM14, GUS15a, KAS16a, NEK16, KAS17, AVA18], since it is a first choice paradigmatic model for the modeling of the dynamics of globally coupled adaptive networks. In particular, it generalizes the Kuramoto (or Kuramoto-Sakaguchi) model with fixed $\kappa$. As it is known from numerical studies performed in Ref. [KAS17], the system (4.1)-(4.2) reaches different cluster states depending on the initial conditions. One-cluster states are a particular kind of state characterized by a frequency synchronized motion of all oscillators. In this chapter, we discuss in detail the nature and properties of these states. We provide necessary and sufficient condition for their existence as well stability and provide insights in the impact of parameter changes.

This chapter includes contents that have been published in [BER19, BER19a]. The structure of this chapter is as follows. In Section 4.1 we prove the existence of one-cluster states for the system (4.1)-(4.2) and provide a complete classification of these states. The stability of oneclusters is rigorously described in the subsequent Section 4.2. Building on these result, the impact of the adaptation rate on the stability is explored, Section 4.3. Finally, we discuss the role of double antipodal states in Section 4.4. All findings of this chapter are summarized in Section 4.5.

\subsection{Classification of one-cluster states}

We start with the collective dynamics, where all oscillators are synchronous up to phase shifts, i.e. $\phi_{i}=s(t)+a_{i}$. It is easy to see from (4.1), that $d s / d t=$ const in this case, and, hence, $s(t)=\Omega t$ with some constant frequency $\Omega$. Moreover, due to the symmetry of system (4.1)-(4.2) with respect to the phase-shift $\phi_{i} \mapsto \phi_{i}-a_{1}$ one can consider $a_{1}=0$ without loss of generality. Therefore, we define the following solutions.

Definition 4.1.1 Phase oscillators $\phi_{i}(t), i=1, \ldots, N$ are said to be

(i) in-phase synchronous if $\phi_{i}(t)=s(t)$ for all $i$;

(ii) antiphase synchronous if $\phi_{i}(t)=s(t)+a_{i}$ with $a_{i} \in\{0, \pi\}$ and there are $i \neq j$ such that $a_{i} \neq a_{j}$;

(iii) rotating-waves if $\phi_{i}(t)=s(t)+(i-1) 2 \pi k / N$, where $k \in\{1, \ldots, N\}$ is the wave number;

(iv) phase-locked if $\phi_{i}=s(t)+a_{i}$ with arbitrary $a_{i} \in \mathbb{T}^{1}$. 
Table 4.1: The table summarizes the values for $n$th order parameter for the phase-locked solutions introduced in Definition 4.1.1.

\begin{tabular}{ll}
\hline State & $n$th order parameter \\
\hline in-phase: $\phi_{i}=\Omega t$ & $R_{n}=1$ \\
anti-phase: $\phi_{i}=\Omega t+a_{i}, a_{i} \in\{0, \pi\}$ & $R_{2 n}=1, R_{2 n+1}=\left|2 \frac{N_{1}}{N}-1\right|$, where $N_{1}$ is the num- \\
& ber of $a_{i}=0$ \\
rotating-wave: $\phi_{i}=\Omega t+i \frac{2 \pi}{N} k, k \neq 0, \frac{N}{2}$ & $R_{n}=1$ if $\frac{n \cdot k}{N} \in \mathbb{N}_{0}$ and $R_{n}=0$ \\
phase-locked: $\phi_{i}=\Omega t+a_{i}, a_{i} \in \mathbb{T}^{1}$ & $R_{n}=\frac{1}{N}\left|\sum_{j=1}^{N} e^{\text {in } a_{j}}\right|$ \\
\hline
\end{tabular}

Note that the following implications hold: rotating-waves with $k=0$ and $k=N / 2$ are in-phase and anti-phase synchronous, respectively. Rotating-waves, in-phase, and antiphase solutions are phase-locked. The term "rotating-wave" relates to the rotating symmetry of the solutions with respect to the spatial coordinate given by the index $i$. These solutions are also known as twisted states [WIL06, GIR12] or splay states [CHO09].

It is straightforward to check the values of the $n$th order parameter (2.15) for the special solutions defined above. For instance, for an in-phase synchronous solution it holds $Z_{n}(\phi(t))=e^{\mathrm{i} n \Omega t}$ and, hence $R_{n}=1$ for all $n \in \mathbb{N}$ and $t \in \mathbb{R}$. For the other solutions the results are summarized in Table 4.1. If $\phi(t)$ is a phase-locked solution, the modulus of the $n$th order parameter does not depend on $\Omega t$, and hence it will be often referred to as $R_{n}(\mathbf{a})$, where $\mathbf{a}=\left(a_{1}, \ldots, a_{N}\right)^{T}$ is the phase shift vector.

With the help of the order parameter we introduce the following three types of phase-locked states.

Definition 4.1.2 Phase oscillators $\phi_{i}(t), i=1, \ldots, N$ are said to form $a$

(i) (Splay cluster) if $R_{2}(\phi)=0$;

(ii) (Antipodal cluster) if $R_{2}(\phi)=1$, i.e., $\phi_{i} \in\{0, \pi\}$ for all $i=1, \ldots, N$;

(iii) (Double antipodal cluster) if $\phi_{i} \in\{0, \pi, \psi, \psi+\pi\}$ for all $i=1, \ldots, N$ with $\psi \in(0, \pi)$.

Note that if $\boldsymbol{\phi}$ is in-phase or anti-phase synchronous, $\boldsymbol{\phi}$ forms an antipodal cluster as well. Rotating waves from Definition 4.1.1 are also known as splay states or incoherent clusters [CHO09]. For all theses rotating waves, it holds $R_{1}(\phi)=R_{2}(\phi)=0$. As it will be shown in Proposition 4.1.1, system (4.1)-(4.2) generically possesses solutions with $R_{2}(\phi)=0$ rather than $R_{1}(\phi)=0$. Both uniformity criteria are clearly related since $R_{2}(\phi)=R_{1}(2 \phi)$. We use the notion splay cluster in a more general sense to stress that the phases are uniformly distributed around the unit circle with respect to the second moment of the order parameter. The following result describes all possible phase-locked solutions in the system of adaptively coupled oscillators (4.1)-(4.2). We call these solutions one-cluster solutions, since all oscillators possess the same frequency.

Proposition 4.1.1 System (4.1)-(4.2) possesses the following phase-locked solutions

$$
\begin{aligned}
\phi_{i} & =\Omega t+a_{i}, \\
\kappa_{i j} & =-\sin \left(a_{i}-a_{j}+\beta\right), \quad i, j=1, \ldots, N
\end{aligned}
$$


(a)

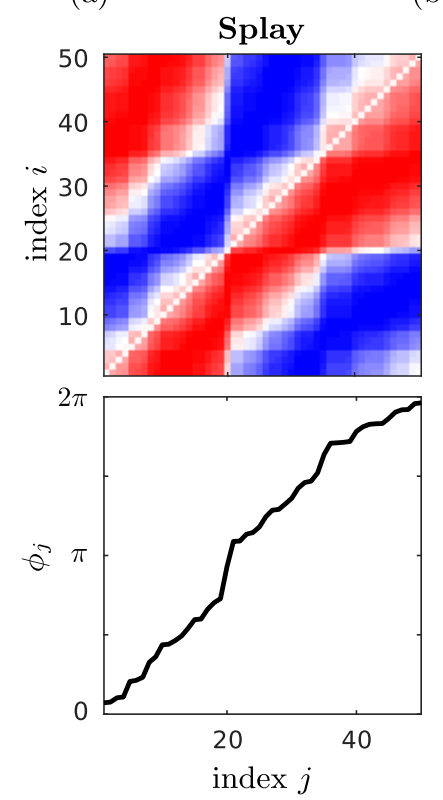

(b)
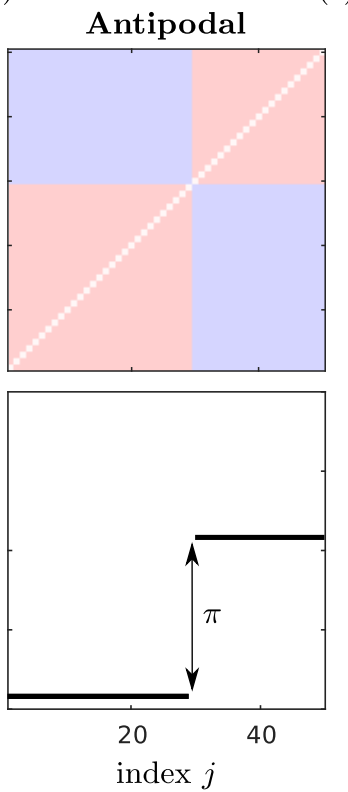

(c)

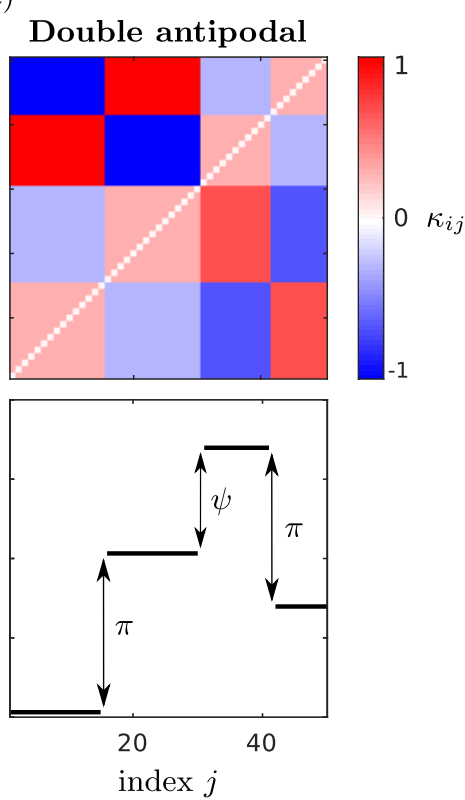

Figure 4.1: Illustration of the three types of one-cluster solutions given by (4.3)-(4.4) for an ensemble of 50 oscillators. One-cluster solutions (a) of splay type $\left(R_{2}(\mathbf{a})=0\right)$ for $\alpha=0.3 \pi, \beta=0.1 \pi$, (b) of antipodal type $\left(R_{2}(\mathbf{a})=1\right)$, for $\alpha=0.2 \pi$, $\beta=-0.95 \pi$ and (c) of double antipodal type satisfying condition (iii) of Proposition 4.1.1 with $m=30$ for $\alpha=0.3 \pi$, $\beta=-0.15 \pi$. Figure taken from [BER19].

if and only if one of the following three conditions is fulfilled:

(i) the phases $a_{i}$ form a splay cluster, i.e., $R_{2}(\mathbf{a})=0$;

(ii) the phases $a_{i}$ form an antipodal cluster, i.e., $R_{2}(\mathbf{a})=1$;

(iii) the phases $a_{i}$ form a double antipodal cluster with $m \in\{1, \ldots, N-1\}, a_{i} \in\left\{0, \pi, \psi_{m}, \psi_{m}+\pi\right\}$, $i=1, \ldots, N$ and $\psi_{m}$ being the unique modulo $2 \pi$ solution to the following equation

$$
\frac{N-m}{m} \sin (\psi-\alpha-\beta)=\sin (\psi+\alpha+\beta),
$$

and the number of phase shifts $a_{i}$ such that $a_{i} \in\{0, \pi\}$ equals to $m$.

The corresponding frequencies are given by

$$
\Omega= \begin{cases}\cos (\alpha-\beta) / 2 & \text { if } R_{2}(\mathbf{a})=0, \\ \sin \alpha \sin \beta & \text { if } R_{2}(\mathbf{a})=1, \\ \frac{1}{2}\left(\cos (\alpha-\beta)-R_{2}(\mathbf{a}) \cos (\psi)\right) & \text { in case (iii). }\end{cases}
$$

The proof of this and other propositions are given in the Appendix A. Note that for the special cases $\alpha=0, \pi$ together with $\beta=\alpha+\pi / 2, \alpha+3 \pi / 2$ solutions with $R_{2}(\mathbf{a}) \notin\{0,1\}$ were discussed in [GUS15a]. Moreover, similar solutions were found in experimental settings with delay-coupled chemical oscillators [BLA13].

Note that conditions (i)-(iii) of Proposition 4.1.1 imply that there are three possible types of one-cluster solutions: splay, antipodal, and double antipodal. We illustrate these solutions in Figs. 4.1(a-c).

In the following, we focus our study mainly on the splay and antipodal clusters with $R_{2}(\mathbf{a}) \in$ 

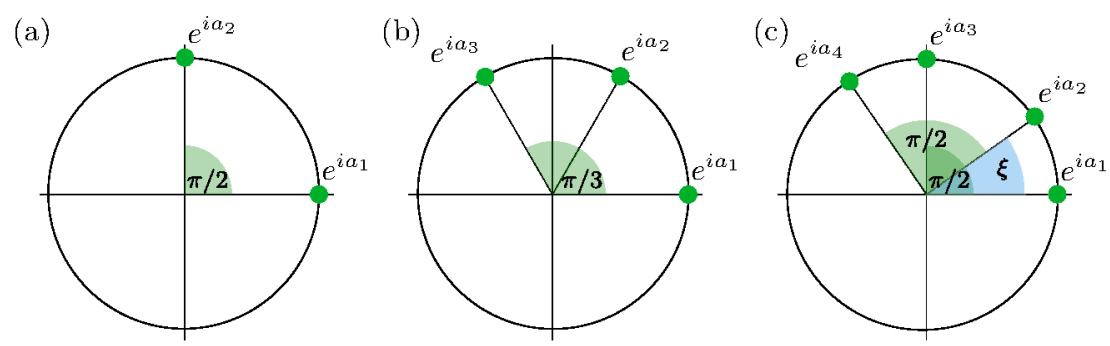

Figure 4.2: Illustration of the family of solutions $S$ (a) $N=2$, (b) $N=3$, (c) $N=4$. Figure taken from [BER19].

$\{0,1\}$, i.e., the phase-locked solutions given by cases (i) and (ii) of Proposition 4.1.1. The role of double antipodal states is discussed in the last section of this chapter. We further remark that the phase-locked solutions (4.3)-(4.4) are relative equilibria with respect to the phase-shift defined in Section 2.3.3, i.e., they are equilibria in the co-rotating frame $\phi \mapsto \phi+\Omega t$.

If $R_{2}(\mathbf{a})=1$, Proposition 4.1.1 implies that $a_{i}$ are either 0 or $\pi$. Therefore, there are $2^{N-1}$ isolated solutions of this kind. Note that the in-phase synchronous solution is an antipodal one-cluster solution.

The situation is different for the splay cluster. The relation $R_{2}(\mathbf{a})=0$ gives the $N-2$ parametric $(N>2)$ family

$$
S:=\left\{\left(\phi_{i}, \kappa_{i j}\right): \phi_{i}=\Omega t+a_{i}, \kappa_{i j}=-\sin \left(a_{i}-a_{j}+\beta\right), \sum_{j=1}^{N} \sin \left(2 a_{j}\right)=\sum_{j=1}^{N} \cos \left(2 a_{j}\right)=0\right\},
$$

where $\Omega=\cos (\alpha-\beta) / 2$. Moreover, analogously to [ASH08], one can show that $S$ is the union of $N-2$ dimensional manifolds.

The structure of the solution family (4.7) is illustrated in Figs. 4.2(a-c) for $N=2,3,4$. Figure 4.2(a) shows one of the two disjoint one-dimensional subsets of $S$ for the case of two adaptively coupled oscillators modulo common rotation of the phases on the circle. In fact, the oscillators have to have a phase shift of $\pi / 2$ in order to meet the condition $R_{2}(\mathbf{a})=0$, i.e., $\mathbf{a}=(\gamma, \gamma+\pi / 2)$ with $\gamma \in[0,2 \pi)$. The dimension of $S$ for $N=2$ is one. For a system consisting of three or four phase oscillators the dimension of $S$ is either 1 or 2, respectively. For $N=3$, one has $\mathbf{a}=$ $(\gamma, \gamma+\pi / 3, \gamma+2 \pi / 3)$, see Fig. 4.2(b). For the case $N=4$, we have $\mathbf{a}=(\gamma, \gamma+\xi, \gamma+\pi / 2, \gamma+\xi+\pi / 2)$ with $\gamma, \xi \in[0,2 \pi)$, see Fig. 4.2(c).

Note that the set of phases satisfying the condition $R_{1}(\mathbf{a})=0$ was described in [ASH08, BUR11, ASH16a]. Our case of splay clusters can be related to this set using the equality $R_{2}(\mathbf{a})=R_{1}(2 \mathbf{a})=$ 0 .

Rotating-waves are a particular case of the splay cluster, namely, the following corollary holds.

Corollary 4.1.2 For any $k \in\{1, \ldots, N-1\}, k \neq N / 2$ the rotating-wave

$$
\begin{aligned}
\phi_{i} & =\Omega t+(i-1) \frac{2 \pi k}{N}, \\
\kappa_{i j} & =-\sin \left((i-j) \frac{2 \pi k}{N}+\beta\right),
\end{aligned}
$$


with $\Omega=\cos (\alpha-\beta) / 2$ is a solution of system (4.1)-(4.2).

Let us make a short remark, which allows for a better understanding and interpretation of the phase-locked solutions given in Proposition 4.1.1. Assume that the phase variables are in a phase-locked solution $\phi_{i}=\Omega t+a_{i}$. Then, the coupling weights $\kappa_{i j}$ have to satisfy the linear system

$$
\dot{\kappa}_{i j}=-\epsilon \kappa_{i j}-\epsilon \sin \left(a_{i}-a_{j}+\beta\right) .
$$

This system has the unique solution $\kappa_{i j}=-\sin \left(a_{i}-a_{j}+\beta\right)$ which is constant, bounded on $\mathbb{R}$, and asymptotically stable as $t \rightarrow \infty$. Therefore, the specific network connectivity $\kappa_{i j}=$ $-\sin \left(a_{i}-a_{j}+\beta\right)$ is associated with a given phase-shift $\mathbf{a}$.

\subsection{Stability of one-cluster states}

In the previous section the existence of one-cluster were discussed. This section is devoted to the analysis of their stability.

In order to study the local stability of one-cluster solutions described in Section 4.1, we linearize the system (4.1)-(4.2) around the solutions (4.3)-(4.4). We obtain the following linearized system

$$
\begin{aligned}
\frac{d}{d t} \delta \phi_{i} & =\frac{1}{N} \sum_{m=0}^{N-1} \sin \left(a_{i}-a_{i+m}+\beta\right) \cos \left(a_{i}-a_{i+m}+\alpha\right)\left(\delta \phi_{i}-\delta \phi_{i+m}\right) \\
& -\frac{1}{N} \sum_{m=0}^{N-1} \sin \left(a_{i}-a_{i+m}+\alpha\right) \delta \kappa_{i(i+m)}, \\
\frac{d}{d t} \delta \kappa_{i(i+m)} & =-\epsilon\left(\delta \kappa_{i(i+m)}+\cos \left(a_{i}-a_{i+m}+\beta\right)\left(\delta \phi_{i}-\delta \phi_{i+m}\right)\right),
\end{aligned}
$$

where we have introduced the new label $m:=j-i$ and the convention $i+m=(i+m) \bmod N$ for convenience. Throughout this paragraph we will make use of the Schur complement [BOY04, LIE15] in order to simplify characteristic equations. More precisely, any $m \times m$ matrix $M$ in the $2 \times 2$ block form can be written as

$$
M=\left(\begin{array}{cc}
A & B \\
C & D
\end{array}\right)=\left(\begin{array}{cc}
\mathbb{I}_{p} & B D^{-1} \\
0 & \mathbb{I}_{q}
\end{array}\right)\left(\begin{array}{cc}
A-B D^{-1} C & 0 \\
0 & D
\end{array}\right)\left(\begin{array}{cc}
\mathbb{I}_{p} & 0 \\
D^{-1} C & \mathbb{I}_{q}
\end{array}\right)
$$

where $A$ is a $p \times p$ matrix and $D$ is an invertible $q \times q$ matrix. The matrix $A-B D^{-1} C$ is called Schur complement. A simple formula for the determinant of $M$ can be derived with the decomposition (4.11)

$$
\operatorname{det}(M)=\operatorname{det}\left(A-B D^{-1} C\right) \cdot \operatorname{det}(D)
$$

This result is important for the subsequent stability analysis. Note that in the following an overline indicates the complex conjugate. 
Lemma 4.2.1 Suppose $\mathbf{a}_{k}=(0,2 \pi k / N, \ldots,(N-1) 2 \pi k / N)^{T}$ with $k \in\{0, \ldots, N-1\}$ and the linear system around the one-cluster solution $\boldsymbol{\phi}=\Omega t \cdot(1, \ldots, 1)^{T}+\mathbf{a}_{k}$ is given by (4.9)-(4.10). Then there exist new coordinates $(\delta \psi, \delta \zeta)$ such that the linearized system can be decomposed into $N$ linear differential equations of the form

$$
\left(\begin{array}{c}
\delta \dot{\psi}_{l} \\
\delta \dot{\zeta}_{l 0} \\
\vdots \\
\delta \dot{\zeta}_{l(N-1)}
\end{array}\right)=C_{l}\left(\begin{array}{c}
\delta \psi_{l} \\
\delta \zeta_{l 0} \\
\vdots \\
\delta \zeta_{l(N-1)}
\end{array}\right) \quad l=0, \ldots, N-1
$$

with

$$
C_{l}:=\left(\begin{array}{cc}
\hat{\lambda}_{l} & b \\
c_{l} & -\epsilon \mathbb{I}_{N}
\end{array}\right),
$$

where, $\mathbb{I}_{N}$ is the $N$-dimensional identity matrix and

$$
\begin{aligned}
\hat{\lambda}_{l} & =\frac{1}{2}\left(\left(Z_{1}\left(\mathbf{a}_{l}\right)-1\right) \sin (\alpha-\beta)-\mathfrak{J}\left(Z_{2}\left(\mathbf{a}_{k}\right)\right) \cos (\alpha+\beta)+\mathfrak{R}\left(Z_{2}\left(\mathbf{a}_{k}\right)\right) \sin (\alpha+\beta)\right) \\
& +\frac{1}{4}\left(\bar{Z}_{1}\left(\mathbf{a}_{2 k-l}\right) \mathrm{i} e^{\mathrm{i}(\alpha+\beta)}-Z_{1}\left(\mathbf{a}_{2 k+l}\right) \mathrm{i} e^{-\mathrm{i}(\alpha+\beta)}\right) . \\
b & =\frac{1}{N}\left(\sin (-\alpha), \ldots, \sin \left((N-1) k \frac{2 \pi}{N}-\alpha\right)\right) \\
c_{l} & =\left(0, \cos \left(k \frac{2 \pi}{N}-\beta\right)\left(1-e^{\mathrm{i} l \frac{2 \pi}{N}}\right), \ldots, \cos \left((N-1) k \frac{2 \pi}{N}-\beta\right)\left(1-e^{\mathrm{i} l(N-1) \frac{2 \pi}{N}}\right)\right)^{T}
\end{aligned}
$$

with any $j \in\{1, \ldots, N\}$.

Proof. Due to the cyclic structure in the equations (4.9) and (4.10) it is possible to decouple them using a discrete Fourier ansatz [PER10c]

$$
\begin{aligned}
\delta \phi_{j} & =\sum_{l=0}^{N-1} e^{\mathrm{i} l j \frac{2 \pi}{N}} \delta \psi_{l}, \\
\delta \kappa_{j(j+m)} & =\sum_{l=0}^{N-1} e^{\mathrm{i} l j \frac{2 \pi}{N}} \delta \zeta_{l m} .
\end{aligned}
$$

Taking this Fourier ansatz and plugging it into the equations (4.9) and (4.10) we get

$$
\begin{aligned}
\sum_{l=0}^{N-1} e^{\mathrm{i} l j \frac{2 \pi}{N}} \delta \psi_{l}= & \frac{1}{N} \sum_{m=0}^{N-1} \sin \left(-m k \frac{2 \pi}{N}+\beta\right) \cos \left(-m k \frac{2 \pi}{N}+\alpha\right) \sum_{l=0}^{N-1} e^{\mathrm{i} l j \frac{2 \pi}{N}}\left(1-e^{\mathrm{i} l m \frac{2 \pi}{N}}\right) \delta \psi_{l} \\
& -\frac{1}{N} \sum_{m=0}^{N-1} \sin \left(-m k \frac{2 \pi}{N}+\alpha\right) \sum_{l=0}^{N-1} e^{\mathrm{i} l j \frac{2 \pi}{N}} \delta \zeta_{l m}, \\
\sum_{l=0}^{N-1} e^{\mathrm{i} l j \frac{2 \pi}{N}} \dot{\delta} \zeta_{l m}= & -\epsilon \sum_{l=0}^{N-1}\left(e^{\mathrm{i} l j \frac{2 \pi}{N}} \delta \zeta_{l m}+\cos \left(-m k \frac{2 \pi}{N}+\beta\right) e^{\mathrm{i} l j \frac{2 \pi}{N}}\left(1-e^{\mathrm{i} l m \frac{2 \pi}{N}}\right) \delta \psi_{l}\right) .
\end{aligned}
$$

After making use of well known trigonometric identities and using the order parameters defined 
in (2.15) we find

$$
\begin{aligned}
\hat{\lambda}_{l} & =\frac{1}{2 N} \sum_{m=0}^{N-1}\left(\sin \left(-\frac{4 \pi}{N} m k+\alpha+\beta\right)-\sin (\alpha-\beta)\right)\left(1-\cos \left(\operatorname{lm} \frac{2 \pi}{N}\right)-\mathrm{i} \sin \left(\operatorname{lm} \frac{2 \pi}{N}\right)\right) \\
& =\frac{1}{2}\left(\left(Z_{1}\left(\mathbf{a}_{l}\right)-1\right) \sin (\alpha-\beta)-\mathfrak{J}\left(Z_{2}\left(\mathbf{a}_{k}\right)\right) \cos (\alpha+\beta)+\mathfrak{R}\left(Z_{2}\left(\mathbf{a}_{k}\right)\right) \sin (\alpha+\beta)\right) \\
& +\frac{1}{4}\left(\bar{Z}_{1}\left(\mathbf{a}_{2 k-l}\right) \mathrm{i} e^{\mathrm{i}(\alpha+\beta)}-Z_{1}\left(\mathbf{a}_{2 k+l}\right) \mathrm{i} e^{-\mathrm{i}(\alpha+\beta)}\right) .
\end{aligned}
$$

The row and the column vectors $b$ and $c_{l}$ can directly be read of from the transformed equation above.

Note that the values $\hat{\lambda}_{l}$ are exactly the eigenvalues for the case where no interaction between the oscillators and their coupling are assumed or the dynamics of the coupling weights are left constant. One might expect that due to the slow-fast dynamics of the system (4.1)-(4.2) a small perturbation in the coupling weights could be neglected for the analysis of stability [AOK11]. In contrast, we show that the local dynamics of the system around the one-cluster solution depends on the interplay between phases and couplings.

Proposition 4.2.2 Suppose $\mathbf{a}_{k}=\left(0, \frac{2 \pi}{N} k, \ldots,(N-1) \frac{2 \pi}{N} k\right)^{T}$ and the linear system around the onecluster solution $\boldsymbol{\phi}=\Omega t \cdot(1, \ldots, 1)^{T}+\mathbf{a}_{k}$ is given by (4.9)-(4.10). Then the Jacobian $J$ of this linearized system possesses the following spectrum

$$
\sigma(J)=\left\{-\epsilon,\left(\lambda_{l ; 1,2}\right)_{l=0, \ldots, N-1}\right\}
$$

with

$$
\lambda_{l ; 1,2}=\frac{\hat{\lambda}_{l}-\epsilon}{2} \pm \frac{1}{2} \sqrt{\left(\hat{\lambda}_{l}+\epsilon\right)^{2}+4 \epsilon(b \cdot c)_{l}}
$$

and $\hat{\lambda}_{l}, b_{l}, c_{l}$ as defined in (4.13), (4.14) and (4.15).

Proof. Using Lemma 4.2.1 we decompose the linear system (4.9)-(4.10) into the $N$ blocks (4.12). Consider now the characteristic polynomial for the $(N+1) \times(N+1)$ matrix $C_{l}$ and assume that $\lambda_{l} \neq-\epsilon$ then by (4.11) we obtain

$$
\operatorname{det}\left(\lambda_{l} \mathbb{I}_{N+1}-C_{l}\right)=\operatorname{det}\left(\begin{array}{cc}
\lambda_{l}-\hat{\lambda}_{l} & -b_{l} \\
-\epsilon c_{l} & \left(\epsilon+\lambda_{l}\right) \mathbb{I}_{N}
\end{array}\right)=\left(\epsilon+\lambda_{l}\right)^{N-1}\left(\left(\epsilon+\lambda_{l}\right)\left(\lambda_{l}-\hat{\lambda}_{l}\right)-\epsilon(b \cdot c)_{l}\right)=0 .
$$

Thus for each $l \in 0, \ldots, N-1$ there are $N-1$ eigenvalues $\lambda_{l}=-\epsilon$. For the two remaining eigenvalues we have to solve the quadratic equation

$$
\lambda_{l}^{2}+\left(\epsilon-\hat{\lambda}_{l}\right) \lambda_{l}-\epsilon \hat{\lambda}_{l}-\epsilon(b \cdot c)_{l}=0
$$

In the case of no weight dynamics or no interdependence between the oscillators and the weights the eigenvalues would read $\lambda_{l ; 1}=\hat{\lambda}_{l}$ and $\lambda_{l ; 2}=-\epsilon$. Therefore, the spectrum would look like $\sigma_{c}=\left\{-\epsilon,\left(\hat{\lambda}_{l}\right)_{l=0, \ldots, N-1}\right\}$ with $(N-1) N$-fold multiplicity for the eigenvalue $-\epsilon$. In contrast to that, 
we get in general $2 N$ eigenvalues that are different from $-\epsilon$ which stem from the interplay of phases and coupling weights. We should further mention that $\hat{\lambda}_{l}\left(\alpha+\frac{\pi}{2}, \beta-\frac{\pi}{2}\right)=(b \cdot c)_{l}(\alpha, \beta)$. With this we write equation (4.17) as

$$
\lambda_{l}^{2}(\alpha, \beta)+\left(\epsilon-\hat{\lambda}_{l}(\alpha, \beta)\right) \lambda_{l}-\epsilon\left(\hat{\lambda}_{l}(\alpha, \beta)+\hat{\lambda}_{l}\left(\alpha-\frac{\pi}{2}, \beta+\frac{\pi}{2}\right)\right)=0 .
$$

The following corollary summarizes the results on the spectrum of the linearized system (4.9)(4.10).

Corollary 4.2.3 Suppose we have $\mathbf{a}_{k}=\left(0, \frac{2 \pi}{N} k, \ldots,(N-1) \frac{2 \pi}{N} k\right)^{T}$ and the linear system (4.9)-(4.10) then

1. (in-phase and anti-phase synchrony) if $k=0$ or $k=N / 2$, the spectrum is given by

$$
\sigma(J)=\left\{(0)_{1},(-\epsilon)_{(N-1) N+1},\left(\lambda_{1}\right)_{N-1},\left(\lambda_{2}\right)_{N-1}\right\}
$$

where $\lambda_{1}$ and $\lambda_{2}$ solve $\lambda^{2}+(\epsilon-\cos (\alpha) \sin (\beta)) \lambda-\epsilon \sin (\alpha+\beta)=0$,

2. (incoherent rotating-wave) if $k \neq 0, N / 2, N / 4,3 N / 4$, the spectrum is

$$
\sigma(J)=\left\{(0)_{N-2},(-\epsilon)_{(N-1) N+1},\left(-\frac{\sin (\alpha-\beta)}{2}-\epsilon\right)_{N-3},\left(\vartheta_{1}\right)_{1},\left(\vartheta_{2}\right)_{1},\left(\bar{\vartheta}_{1}\right)_{1},\left(\bar{\vartheta}_{2}\right)_{1}\right\}
$$

where $\vartheta_{1}$ and $\vartheta_{2}$ solve $\vartheta^{2}+\left(\epsilon+\frac{1}{2} \sin (\alpha-\beta)-\frac{1}{4} \mathrm{i} e^{\mathrm{i}(\alpha+\beta)}\right) \vartheta-\frac{\epsilon}{2} \mathrm{i} e^{\mathrm{i}(\alpha+\beta)}=0$,

3. (4-rotating-wave solution) if $k=N / 4,3 N / 4$, the spectrum is

$$
\sigma(J)=\left\{(0)_{N-1},(-\epsilon)_{(N-1) N+1},\left(-\frac{\sin (\alpha-\beta)}{2}-\epsilon\right)_{N-2},\left(\lambda_{1}\right)_{1},\left(\lambda_{2}\right)_{1}\right\}
$$

where $\lambda_{1}$ and $\lambda_{2}$ solve $\lambda^{2}+(\epsilon+\sin (\alpha) \cos (\beta)) \lambda+\epsilon \sin (\alpha+\beta)=0$.

Here, the multiplicities for each eigenvalue are given as lower case labels.

As we can see from this corollary there exists always at least one zero eigenvalue. This is due to the phase-shift symmetry of (4.1)-(4.2) we already discussed in Section 4.1. The additional zero eigenvalues for the wave numbers $k \neq 0, N / 2$ can be explained with our findings from Proposition 4.1.1 and Corollary 4.1.2. These linear rotating-wave solutions belong to a $N-2$ dimensional family of solutions characterized by $R_{2}(\mathbf{a})=0$. Thus, around any point of this family the linear equation

$$
\sum_{j=1}^{N} e^{i 2 a_{j}} \delta \phi_{j}=0
$$

holds for a certain choice of coordinates $\left\{\delta \phi, \delta \kappa_{i j}\right\}$, and hence there are two linearly independent equations for the infinitesimal perturbations $\delta \phi_{i}$. This explains the appearance of $N-2$ zero eigenvalues. They correspond to the variation along the manifold of solution. In the special case of $k=N / 4,3 N / 4$ the two algebraic equations (4.18) are linear dependent and we are thus left with only one linear equation which increases the multiplicity of the zero eigenvalue by one.

The results of Corollary 4.2.3 are presented in Fig. 4.3(a-c) and compared with numerical 
(a)

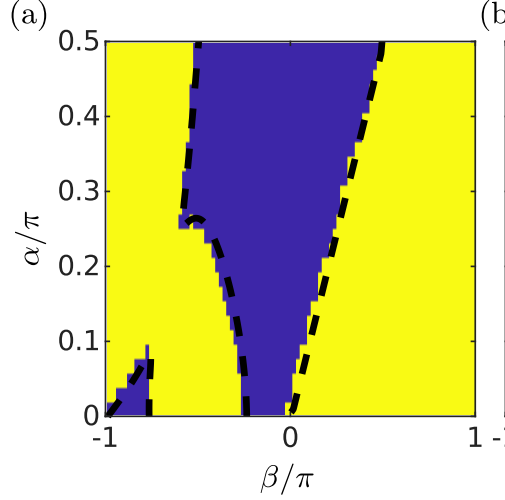

(b)

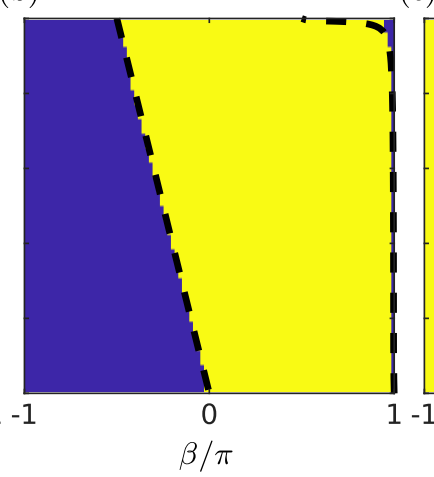

(c)

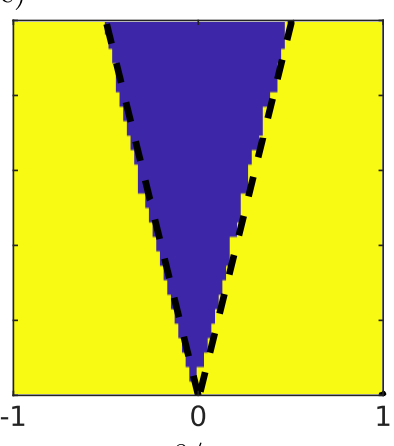

Figure 4.3: Stability diagrams for rotating-wave clusters depending on the parameters $\alpha$ and $\beta$ are shown. The regions are colored according to numerical simulation. Blue regions correspond to stable solutions while yellow regions correspond to unstable solutions. The black dashed lines show to the borders of stability determined by Corollary 4.2.3. Parameter $\epsilon=0.01$ is fixed fo all simulations. (a) $k=1$, (b) $k=N / 2$, (c) $k=N / 4$. Figure taken from [BER19].
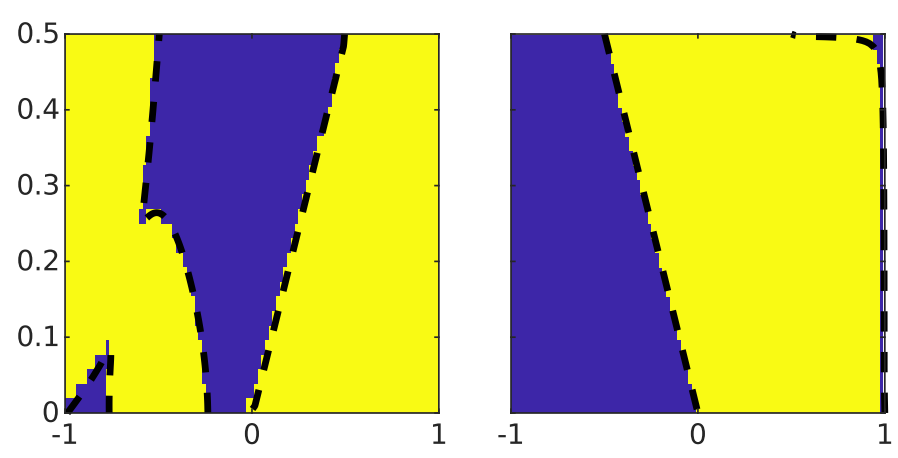

Figure 4.4: Stability diagram for splay and antipodal one-cluster solutions depending on the parameters $\alpha$ and $\beta$ are shown. The regions are colored according numerical eigenvalues of the Jacobian 4.9-4.10. Blue areas correspond to stable while yellow areas correspond to unstable regions. Parameter $\epsilon=0.01$ is fixed in all simulations. (a) Splay solution as in Fig. 4.1(a), (b) Anti-phase solution as in Fig. 4.1(b). Figure modified from [BER19].

simulations. The numerical results are obtained by numerical integration of system (4.1)-(4.2) with $N=20$. The initial conditions for each simulation are set to the one-cluster solution given in Proposition 4.1.1 with a small perturbation added to each dynamical variable and randomly chosen from the interval $[-0.01,0.01]$. The numerical integration is stopped after $t=5000$ time steps. The relative coordinates $\Theta_{i}:=\phi_{i}-\phi_{1}$ for $i=1, \ldots, N$ are introduced in order to compare the initial phase configuration with the distribution of the phases after numerical integration. A onecluster is said to be stable if $\boldsymbol{\Theta}$ after numerical integration is closer to the theoretical one-cluster state than $\boldsymbol{\Theta}$ of the initially perturbed phase distribution. Otherwise, the one-cluster solution is considered as unstable. Closeness is measured by the Euclidean distance. The parameter regions in the $(\alpha, \beta)$ plane for stable one-cluster solutions are colored blue while the regions for unstable one-cluster solutions are colored yellow. The black dashed lines correspond to the borders of stability determined with the results in Corollary 4.2.3. In particular, a state is asymptotically stable if $\mathfrak{R}(\lambda)<0$ for all $\lambda \in \sigma(C)$ except the zero eigenvalues related to the perturbations along the solution families. In all three cases the numerical and analytic results agree very well.

In addition to the analysis of rotating-wave solutions, we investigate the stability for the splay solutions characterized by $R(\mathbf{a})=0$ and the antipodal solutions characterized by $R(\mathbf{a})=1$. For 
this, the stability is calculated semi-analytically by taking the solutions displayed in Fig. 4.1(ab), plugging them into the Jacobian matrix given by the linearized equations (4.9)-(4.10) and determining the eigenvalues of the Jacobian numerically. The results of this procedure are shown in Fig. 4.4 together with the borders of stability calculated with Proposition 4.1.1. In comparison with Fig. 4.3, the analysis yields the same stability regions which are found for the rotating-wave solutions. The numerical findings indicate that the stability for all splay and antipodal solutions coincide with the stability of the rotating-waves.

Complementary to the previous stability results, all cases of antipodal, 4-phase, and double antipodal cluster are analyzed. For these states the analysis is slightly more subtle and given in the appendix A.2. In the following Proposition, we summarize the findings.

Proposition 4.2.4 The following statements hold true.

1. The set of eigenvalues of the linearized system (4.9)-(4.10) around all antipodal states with $a_{i} \in$ $\{0, \pi\}$ agrees with the set $L$ in Prop. 4.2.2 for rotating-wave states with $k=0, N / 2$.

2. The set of eigenvalues to the linearized system (4.9)-(4.10) around all 4-phase-cluster states with $a_{i} \in\{0, \pi / 2, \pi, 3 \pi / 2\}$ and $R_{2}(\boldsymbol{a})=0$ agrees with the set $L$ in Prop. 4.2.2 for 4-rotating-wave states.

3. The double antipodal states are unstable for all $\alpha$ and $\beta$.

As indicated by the numerical analysis in Fig. 4.4, Proposition 4.2.4 shows that in fact in- as well as anti-phase cluster share the same stability features as all other antipodal clusters. In addition, also general 4-phase cluster possess the same Lyapunov spectrum as their special 4-rotating wave cluster.

Remarkably, the third type of one-cluster, namely, the double antipodal state is unstable everywhere in parameter space. In Section 4.4, we describe the role of double antipodal state for the global structure of the phase space.

\subsection{Adaptation rate dependence of one-cluster stability}

In the previous sections, the existence and stability of one-cluster solutions are rigorously described. Building on this, we analyze dependence of the one-cluster states on the adaptation rate $\epsilon$. In contrast to the existence of one-cluster states, the stability of those states depend crucially on the time-separation parameter.

The diagram in Fig. 4.5 shows the regions of stability for antipodal and rotating-wave onecluster states. The diagram is based on the analytic results presented in Corollary 4.2.3 and Proposition 4.2.4.

In Fig. 4.5 the regions of stability are presented for several values of the time separation parameter $\epsilon$. The first case in panel (a) assumes $\epsilon=0$, where the network structure is non-adaptive but fixed to the values given by the one-cluster states, i.e., $\kappa_{i j}=-\sin \left(a_{i}-a_{j}+\beta\right)$ as given in Section 4.1. 


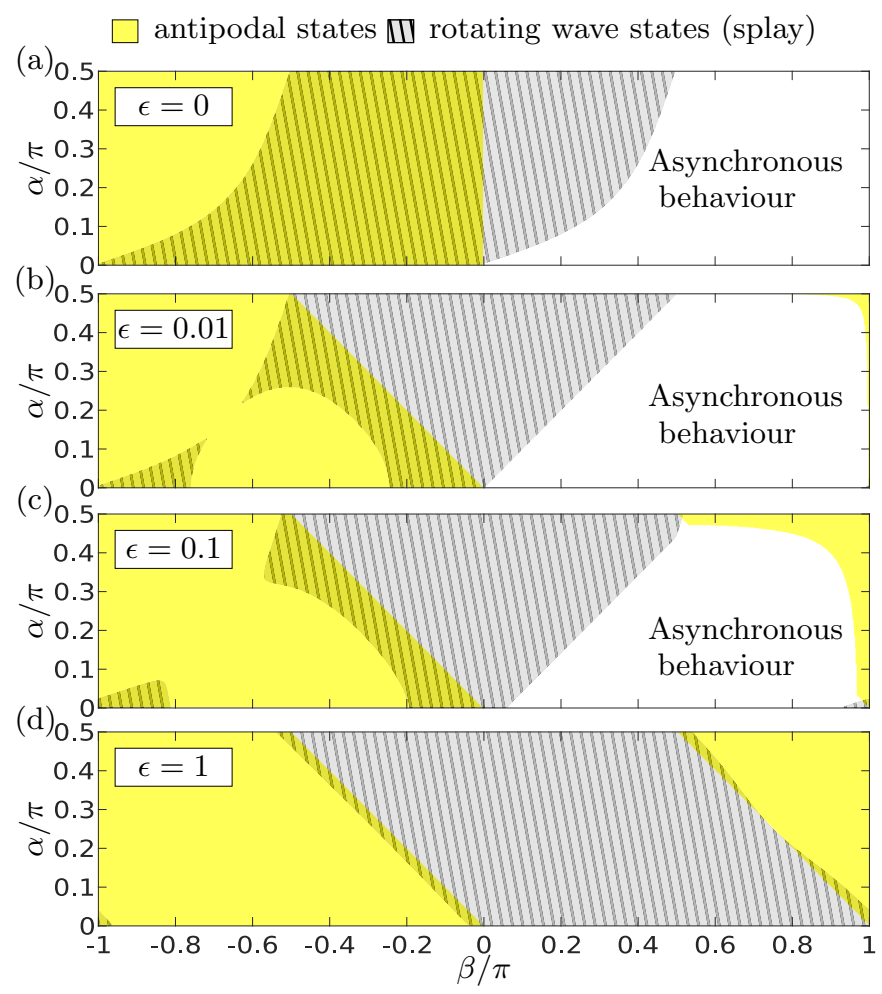

Figure 4.5: The regions of stability for antipodal and rotating-wave states are presented in $(\alpha, \beta)$ parameter space for different values of $\epsilon$. Coloured and hatched areas refer to stable regions for these states as indicated in the legend. White areas refer to region where these one-cluster states are unstable. (a) $\epsilon=0$; (b) $\epsilon=0.01$; (c) $\epsilon=0.1$; (d) $\epsilon=1$. Figure taken from [BER19a].

The linearized system in this case is given by

$$
\frac{\mathrm{d} \delta \phi_{i}}{\mathrm{~d} t}=-\frac{1}{2 N} \sum_{j=1}^{N}\left(\sin (\alpha-\beta)-\sin \left(2\left(a_{i}-a_{j}\right)+\alpha+\beta\right)\right)\left(\delta \phi_{i}-\delta \phi_{j}\right) .
$$

For the synchronized or antipodal state, the value $2 a_{i} \bmod 2 \pi$ is the same for all $i$. Hence, the term $2\left(a_{i}-a_{j}\right)$ disappears and the linearized system (4.19) possesses the same form as the linearized system for the synchronized state of the Kuramoto-Sakaguchi system [BUR11] with coupling constant $\sigma(\beta)=-\sin (\beta)$. As it follows from Ref. [BUR11], the synchronized as well as all other antipodal states are stable for $\sigma(\beta) \cos (\alpha)>0$. The region of stability of the rotating-wave cluster has a more complex shape, see hatched area in Fig 4.5(a). We find large areas where both types of one-cluster states are stable simultaneously, as well as the regions where no frequency synchronized state is stable. The results shown in Fig 4.5(a) are in agreement with Ref. [AOK11], where the authors consider the case $\epsilon=0$ in order to approximate the limit case of extremely slow adaptation $\epsilon \rightarrow 0$. However, such an approach for studying the stability of clusters for small adaptation is not correct in general. As Figs. 4.5(b-d) show, the stability of the network with small adaptation $\epsilon>0$ is different.

The case $\epsilon=0.01$ is shown in Fig 4.5(b), where we observe regions for stable antipodal and rotating-wave states as well. The introduction of a small but non-vanishing adaptation changes the regions of stability significantly. The diagram in Fig 4.5(b) remains qualitatively the same for smaller values of $\epsilon$. This can be read of from the analytic findings presented in the Corollary 4.2.3 and Proposition 4.2.4. The changes in the stability areas are due to subtle changes in the equation 
which determine the eigenvalue of the corresponding linearized system. In fact, the adaptation introduces the necessary condition $\sin (\alpha+\beta)<0$ for the stability of antipodal states. Additionally, for all rotating-wave states of splay type, the necessary condition $\sin (\alpha-\beta)+2 \epsilon>0$ is introduced, see Corollary 4.2.3. This is why, a non-trivial effect of adaptivity on the stability in Figs. 4.5 is observed. In particular, the parameter $\beta$, which determines the plasticity rule, has now a non-trivial impact on the stability of antipodal states for any $\epsilon>0$. As it can be seen in Fig. 4.5(b), one-cluster states of antipodal type are supported by a Hebbian-like adaptation $(\beta \approx-\pi / 2)$ while splay states are supported by causal rules $(\beta \approx 0)$. For the asynchronous region, the dynamical system (4.1)-(4.2) can exhibit very complex dynamics and show chaotic motion [KAS16a]. This region is supported by an anti-Hebbian-like rule $(\beta \approx \pi / 2)$.

By increasing the parameter $\epsilon$, see Fig. 4.5(c,d), two observations can be made. First, the region of asynchronous dynamical behavior is shrinking. For $\epsilon=1$, we find at least one stable one-cluster state for any choice of the phase lag parameters $\alpha$ and $\beta$. Secondly, the regions where both types of one-cluster states are stable are shrinking as well. In the limit of instant network adaptation, i.e., $\epsilon \rightarrow \infty$, the stability regions are completely separated. Both types of one-cluster states divide the whole parameter space into two areas. In this case, the boundaries are described by $\alpha+\beta=0$ and $\alpha+\beta=\pi$. This division can be seen from the analytic findings presented in Corollary 4.2.3. In the case of antipodal states, the quadratic equation which determines the Lyapunov coefficients has negative roots if and only if $\epsilon+\cos (\alpha) \sin (\beta)>0$ and $\sin (\alpha+\beta)<0$. Here, even for $\epsilon>1$, the condition $\sin (\alpha+\beta)<0$ is the only remaining one. Similarly, we find $\sin (\alpha+\beta)>0$ as a condition for the stability of rotating-wave states for $\epsilon \rightarrow \infty$.

\subsection{Double antipodal states}

Splay and antipodal clusters serve as building blocks for multicluster states. The third type, the double antipodal clusters, are not of this nature since they appear to be unstable everywhere. As unstable objects, they can still play an important role for the dynamics. Here we would like to present an example, where the double antipodal clusters become part of a simple heteroclinic network. As a result, they can be observed as metastable states in numerics.

As an example, we first analyze the system of $N=3$ adaptively coupled phase oscillators which is the smallest system with a double antipodal state. According to the definition of a double antipodal state, laid out in Section 4.1, the phases $a_{i}$ of the oscillators $\phi_{i}$ are allowed to take values from the set $\{0, \pi, \psi, \psi+\pi\}$ where $\psi$ uniquely solves Eq. (4.5) for a given $m \in\{1, \ldots, N\}$. Further, at least one oscillator $\phi_{i}$ with $a_{i} \in\{0, \pi\}$ and one oscillator $\phi_{j}(j \neq i)$ with $a_{j} \in\{\psi, \psi+\pi\}$ are needed in order to represent one of the two antipodal groups. Note that for the parameters given in Fig. 4.6 and $N=3$, the equation (4.5) yields $\psi=1.602 \pi$ if $a_{1}, a_{2} \in\{0, \pi\}$ and $a_{3} \in\{\psi, \psi+\pi\}$.

In Fig. 4.6(a) we present trajectories which initially start close to antipodal clusters. The trajectories in phase space are represented by the relative coordinates $\theta_{12}=\phi_{1}-\phi_{2}$ and $\theta_{13}=\phi_{1}-\phi_{3}$. In particular, the two configurations with $\left(\theta_{12}=0, \theta_{13}=0\right)$ and $\left(\theta_{12}=0, \theta_{13}=\pi\right)$ are considered. The coupling weights are initialized according to Eq. (4.4). With the given parameters, the unstable manifold of the antipodal state is one-dimensional which can be determined via Corollary 4.2.2. For the numerical simulation, we perturb the antipodal state in such a way that two distinct orbits close to the unstable manifold are visible. For both configuration the two 
orbits are displayed in Fig. 4.6(a). It can be observed that after leaving the antipodal state the trajectories approach the double antipodal states before leaving it towards the direction of a splay state. With this we numerically find orbits close to "heteroclinic", which connect antipodal, double antipodal, and splay clusters, see schematic picture on the right in Fig. 4.6(b). The phase differences $\theta_{12}$ and $\theta_{13}$ at the double antipodal state agree with the solution $\psi$ of Eq. (4.5) or $\psi+\pi$.

Figure 4.6(b) further justifies our statements on the heteroclinic contours. Here, we see the time series for the second moment order parameter for all trajectories in Fig. 4.6(b). It can be seen that in all cases we start at an antipodal cluster $\left(R_{2}(\phi)=1\right)$ from which the double antipodal state $\left(R_{2}(\phi) \approx 0.447\right.$, theoretical $)$ is quickly approached. The trajectories stay close to the double antipodal cluster for approximately 2000 time units (shaded area) before leaving the invariant set towards the splay state $\left(R_{2}(\phi)=0\right)$.

As a second example we analyze a system of $N=100$ adaptively coupled phase oscillators. Here, we choose two particular antipodal states as initial condition and add a small perturbation to both. One of the states is chosen as an in-phase synchronous cluster. In both cases, the couplings weights are initialized in accordance with Eq (4.4). For Figure 4.6(c) we depict the trajectories which show a clear heteroclinic contour between antipodal, double antipodal, and splay state as in the example of three phase oscillators. We illustrate the heteroclinic connections and present $R_{2}(t)$ for the corresponding trajectories in Fig. 4.6(c). Here, the zoomed view clearly shows that the trajectories for both initial conditions starting at an antipodal cluster $\left(R_{2}(\phi)=1\right)$ again first approach a double antipodal state $\left(R_{2}(\phi) \approx 0.990\right.$, theoretical) before leaving it towards a splay state $\left(R_{2}(\phi)=0\right)$. More precisely, each trajectory comes close to a particular double antipodal state for which only one oscillator has a phase in $\{\psi, \psi+\pi\}$. Remarkably, these states, also known as solitary states, have been found in a range of other systems of coupled oscillators, as well [MAI14a]. With the results in Proposition A.2.3 in the Appendix, one can show that this double antipodal states have a stable manifold with co-dimension one which thus divides the phase space. Next to this fact, numerical evidence for the existence of heteroclinic connections between antipodal and double antipodal states as well as between double antipodal and the family of splay states is provided in Fig. 4.6(c). With this, double antipodal states play an important role for the organization of the dynamics in system (4.1)-(4.2). 

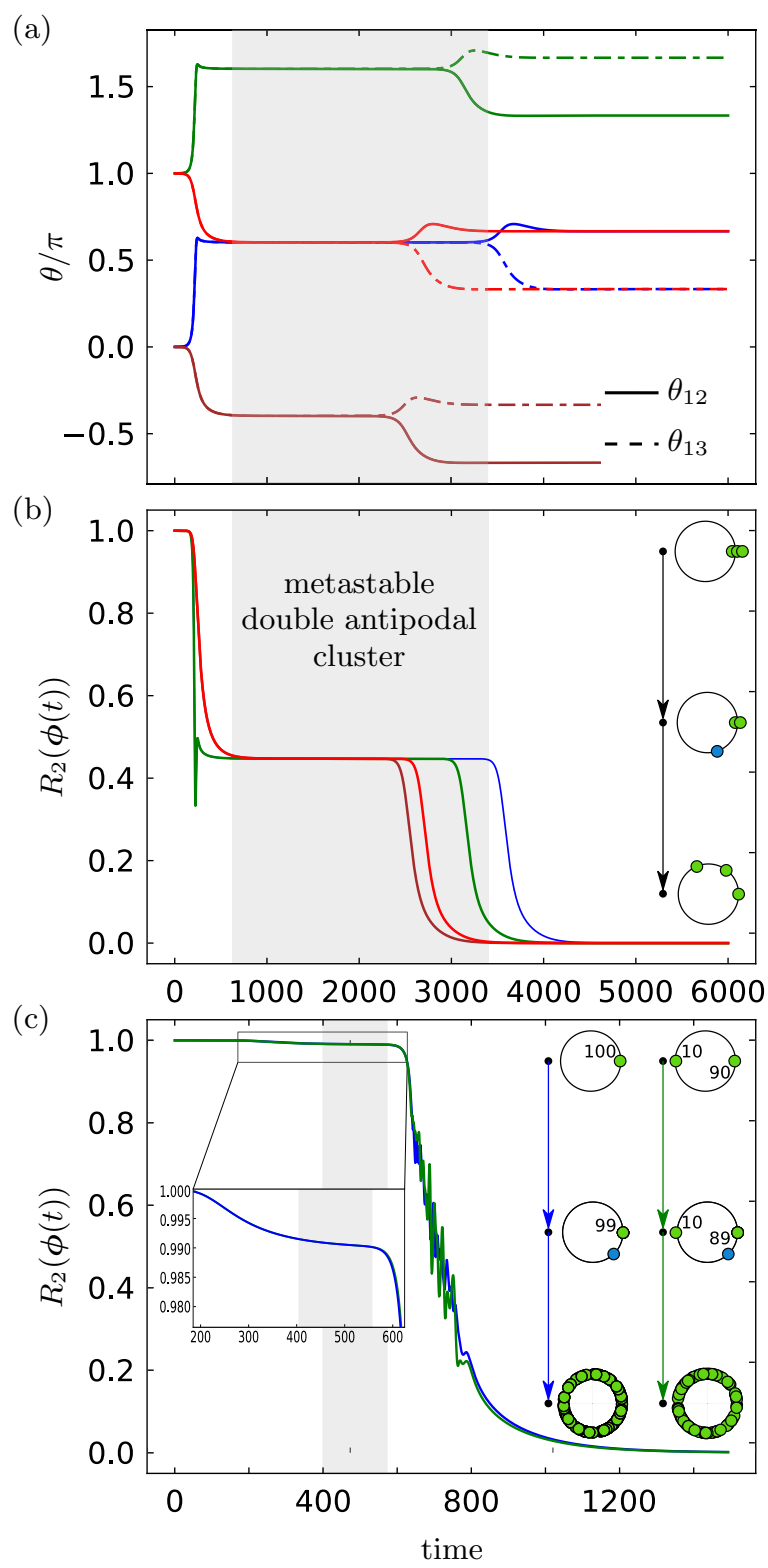

Figure 4.6: Heteroclinic orbits between several steady states in a system of 3 and 100 adaptively coupled phase oscillators. (a) The time series for the relative phases $\theta_{12}$ (solid lines) and $\theta_{13}$ (dashed lines) for $N=3$ are shown. Lines with the same colour correspond to the same trajectories. Panel $(b, c)$ show time series for the second moment order parameter $R_{2}(\phi(t))$ as well as a schematic illustration of the observed heteroclinic connections (right) for (b) $N=3$ and (c) $N=100$. Parameter values: $\epsilon=0.01, \alpha=0.4 \pi$, and $\beta=-0.15 \pi$. Figure taken from [BER19a]. 


\subsection{Summary}

The numerical analysis in Ref. [KAS17] suggested that one-cluster states in system (4.1)-(4.2) may serve as building blocks for multicluster states. In this chapter, we have exhaustively analyzed the properties of one-cluster states in a network of adaptively coupled phase oscillators. In particular, we have found that there are only three types of one-cluster states: splay, antipodal, and double antipodal. It has been shown that all one-cluster solutions of splay type form an $N-2$ dimensional family and thus give rise to infinitely many solutions the system can achieve. In order to understand the stability of these cluster states, we have performed a linear stability analysis. We have provided analytic results for the stability of antipodal as well as of special types of splay states, namely, rotating-wave states. The stability of these states is rigorously described, and the impact of all parameters has been shown. Note that due to the $N-2$ dimensional family of splay type cluster, the rotating-wave states possess $N-2$ neutrally stable directions. Remarkably, this property of splay states has been also found in networks of pulse-coupled rotators [CAL09a] and excitable neurons [DIP12].

In this chapter, we have seen that ring-like structures are dynamically associated with rotatingwave and splay states. Furthermore, ring networks are important motifs in neural networks [COM03, SPO11, POP11] and have been the basis for many interesting dynamical regimes found for different systems, e.g. [ABR04, KLI15, JAR18, OME19c]. Moreover, ring-structures were recently found in fly brains [KIM17a]. In our study, we have presented how ring-like structures could emerge due to adaptation. In particular, we have shown that causal plasticity rules, see also Fig. 3.1, as they are found in many experimental studies [ABB00, BI01, CAP08a] support the appearance of ring-like networks.

While the time-scale separation has no influence upon the existence of the one-cluster states found in this study, it plays an important role for the stability of the one-clusters. The regions of stability in parameter space have been presented for different choices of the time-scale separation parameter. The singular limit $(\epsilon \rightarrow 0)$ and the limit of instantaneous adaptation have been analyzed. The latter shows that the stability regions of the splay and the antipodal states divide the whole space into two equally sized regions without intersection. Instantaneous adaptation cancels multistability of these states. The consideration of the singular limit shows that it differs from the case of no adaptation. Therefore, even for very slow adaptation, the oscillatory dynamics alone is not sufficient to describe the stability of the system.

Additionally to the analysis of splay and antipodal clusters, we have proved that the double antipodal states are unstable in the whole parameter range. In fact, they appear to be saddlepoints in the phase space. We have found that in a system of 3 oscillators, double antipodal states are transient states in a small heteroclinic network between antipodal and splay states. They appear to be metastable, i.e., observable for a relatively long time and therefore are physically important transient states. Moreover, an additional analysis for an ensemble of 100 phase oscillators has revealed the importance of the double antipodal states for the global dynamics of the whole system. 



\section{Multicluster states in adaptive networks of coupled phase oscillators}

In this chapter we investigate the shape and properties of frequency-cluster (multicluster) states as they have been found in Chapter 3, i.e., where each cluster has a different frequency. Inspired by the reduced phenomenological model presented in Chapter 3, we have studied a paradigmatic model of adaptively and globally coupled phase oscillator in Chapter 4 which reads

$$
\begin{aligned}
\frac{d \phi_{i}}{d t} & =\omega-\frac{1}{N} \sum_{j=1}^{N} \kappa_{i j} \sin \left(\phi_{i}-\phi_{j}+\alpha\right), \\
\frac{d \kappa_{i j}}{d t} & =-\epsilon\left(\kappa_{i j}+\sin \left(\phi_{i}-\phi_{j}+\beta\right)\right),
\end{aligned}
$$

see also Section 2.3.3. In Chapter 4, the properties of one-cluster state have been exhaustively analyzed. In this chapter we study numerically as well as analytically multicluster states which we observe by solving the equations (5.1)-(5.2) From the numerical study in Ref. [KAS17], we know that certain phase-locked solutions may serve as building blocks for (hierarchical) multicluster solutions. Here, we show that indeed multiclusters are build-up by one-cluster states and even may inherit their dynamical features. Multicluster states are formally defined as follows.

Definition 5.0.1 Phase oscillators $\phi_{i}(t)$ form a multicluster if they can be separated into $M$ groups of phase-locked oscillators (clusters), i.e., for all $\mu \in\{1, \ldots, M\}$ the phase oscillators $\phi_{i, \mu}, i \in\left\{1, \ldots, N_{\mu}\right\}$, from each group $\mu$ satisfy $\phi_{i, \mu}(t)=s_{\mu}(t)+a_{i, \mu}$.

The appearance of multiclusters is interesting and nontrivial, since such solutions, in contrast to one-clusters, are no more relative equilibria of (5.1)-(5.2), but are periodic or quasi-periodic solutions, which appear due to the special structure of the equation and adaptive nature of the coupling. The oscillators within one cluster possess a synchronized temporal dynamics with possible phase lags. In a multicluster, the coupling matrix $\kappa$ is divided into different blocks according to the division by clusters: $k_{i j, \mu \nu}$ will refer to the coupling weight between the $i$ th oscillator of cluster $\mu$ to the $j$ th oscillator of cluster $v$.

This chapter includes contents that have been published in [BER19, BER19a]. The chapter is organized as follows. First, in Section 5.1, system (5.1)-(5.2) is numerically analyzed and all cluster multicluster states are qualitatively described and classified. Subsequently, we derive analytic expression for all types of multicluster states. The results are given and exhaustively discussed in Sections 5.2, 5.4, and 5.5 for splay, antipodal, and mixed type multicluster states, respectively. Additionally, the main existence theorem for multicluster states, particularly for antipodal and mixed type, is given in Section 5.3. In Section 5.6, we analyze numerically as well as analytically the stability of multicluster states and discuss the hierarchical structure of cluster sizes. All findings of this chapter are summarized in Section 5.7. 
(a)

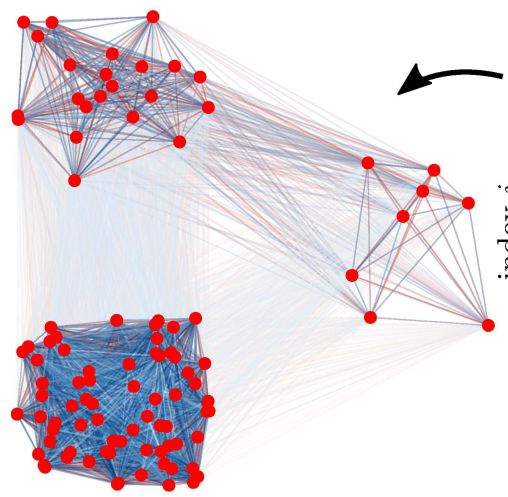

Coupling network

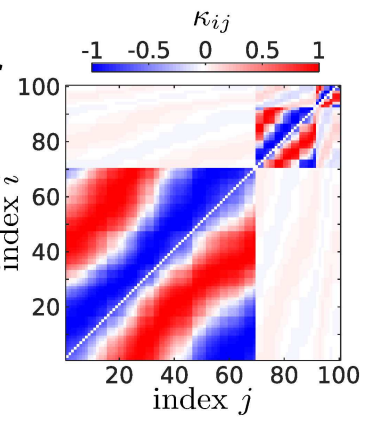

(b) Oscillator phases

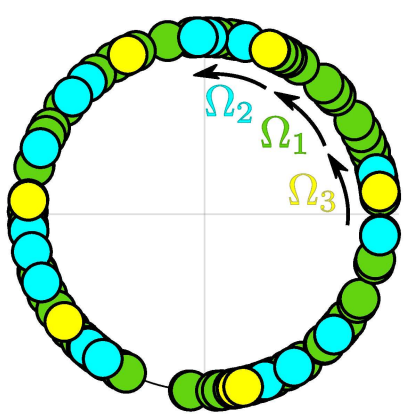

Figure 5.1: Three-frequencycluster of splay type at $t=10000$. (a) Coupling weights represented as a graph (left) and as a coupling matrix (right). In the graph representation, the dynamical nodes are represented by red nodes and the edges are coloured with respect to the coupling weight. Red and blue refer to positive and negative coupling weights, respectively. Light and dark colors refer to weak and strong coupling weights, respectively. (b) Distribution of the phases $\phi_{i}$ for each of the three clusters. Each node represents one oscillator and is coloured with respect to the cluster to which it belongs. Parameter values: $\epsilon=0.01, \alpha=0.3 \pi, \beta=0.23 \pi, \omega=0$, and $N=100$. Figure modified from [BER19a].

\subsection{Numerical observation of multicluster states}

Figure 5.1 shows a hierarchical multicluster state. The solution was obtained by integrating the system (5.1)-(5.2) numerically starting from uniformly distributed random initial conditions. The self-couplings $\kappa_{i i}$ are set to zero in numerical simulations, since they do not influence the relative dynamics of the system. This is due to the fact that all variables $\kappa_{i i}$ converge to $-\sin \beta$, which leads to the same constant term $\sin (\beta) \sin (\alpha) / N$ in the right hand side of Eq. (5.1). The latter term can be absorbed in the co-rotating coordinate frame. We reorder the oscillators (after sufficiently long transient time) by first sorting the oscillators with respect to their average frequencies. After that the oscillators with the same frequency are sorted by their phases. Figure 5.1(a) displays the coupling matrix (right) of the multicluster state and a representation of the coupling structure as a network graph (left). The coupling matrix demonstrates a clear splitting into three groups. This splitting is also visible in the graph representation of the coupling network. The coupling weights between oscillators of the same group vary in a larger range than between those of different groups which are generally smaller in magnitude. The splitting into three groups is manifested in the behaviour of the phase oscillators, as well. We find that the oscillators of the same group possess the same constant frequency with possible phase lags, Fig.5.1(b). We call the groups of oscillators (frequency) clusters and the corresponding dynamical states multi(frequency)cluster states.

In a multicluster, the coupling matrix $\kappa$ is divided into different blocks. We observe that the behavior for each oscillator in a $M$-cluster state takes the form

$$
\phi_{i, \mu}(t)=\Omega_{\mu} t+a_{i, \mu}+s_{i, \mu}(t) \quad \begin{array}{ll}
\mu=1, \ldots, M \\
& i=1, \ldots, N_{\mu}
\end{array}
$$

where $M$ is the number of clusters, $N_{\mu}$ is the number of oscillators in the $\mu$-th cluster, $a_{i, \mu} \in[0,2 \pi)$ are phase lags, and $\Omega_{\mu} \in \mathbb{R}$ is the collective frequency of the oscillators in the $\mu$-th cluster. The functions $s_{i, \mu}$ are bounded. 
The numerical analysis of system (5.1)-(5.2) shows the appearance of different multicluster states depending on particular choices of the phase lag parameters $\alpha$ and $\beta$ as well as on initial conditions. Starting from uniformly distributed random initial conditions the system end up in several states such as multiclusters and chimera-like states [KAS17]. Figure 5.2 shows examples for the three types of multicluster states which appear dynamically in (5.1)-(5.2).

\subsubsection{Splay type cluster states}

The first type is called splay type multicluster state, see Fig. 5.2(a). The separation into three clusters is clearly visible in the coupling matrix, as well as a hierarchical structure in the cluster sizes. Regarding the distribution of the phases, we notice that the oscillators from each group are almost homogeneously dispersed on the circle. In fact, the phases from each cluster fulfill the condition $R_{2}\left(\phi_{\mu}\right)=0(\mu=1,2,3)$ with order parameter (2.15). Note that splay states as they are defined in several other works [NIC92, STR93a, CHO09] share the property $R_{1}(\phi)=0$. This property can be seen as a measure of incoherence for the oscillator phases, as well. In fact, it was shown that splay states are part of a whole family of solutions [BUR11, ASH16a] given by exactly $R_{1}(\phi)=0$. Further, $R_{2}(\phi)=R_{1}(2 \phi)$ relates the two measures of incoherence. These facts motivate the definition of those clusters with $R_{2}(\phi)=0$ as splay type clusters.

The temporal behavior for all phase oscillators in the splay multicluster state is characterized by a constant frequency which differs for the different clusters, i.e., according to (5.3), $\phi_{i, \mu}(t)=$ $\Omega_{\mu} t+a_{i, \mu}$ with $R_{2}\left(\boldsymbol{a}_{\mu}\right)=0$ for all $\mu=1,2,3$ and $i=1, \ldots, N_{\mu}$. In addition, the hierarchical cluster sizes are reflected in the frequencies. Oscillators of a big cluster have a higher frequency than those of smaller clusters. The coupling weights between the phase oscillators are fixed or change periodically with time depending on whether the oscillators belong to the same or different clusters, respectively. Moreover, the amplitude of coupling weights between clusters depends on the frequency difference of the corresponding clusters. The higher the frequency difference, the smaller is the amplitude. The periodic behavior of the coupling weights between clusters is present in all types of multicluster states (Fig. 5.2(a,b,c)).

\subsubsection{Antipodal type cluster states}

Figure 5.2(b) shows another possible multicluster state. As in Fig. 5.2(a) the clusters are clearly visible and their oscillators show frequency synchronized temporal behavior. In addition, the time series for the oscillators show periodic modulations on top of the linear growth. This additional dynamics is the same for all oscillators of the same cluster, and hence they are still temporally synchronized. We have $\phi_{i, \mu}(t)=\Omega_{\mu} t+a_{i, \mu}+s_{\mu}(t)$. In analogy to the coupling weights between the clusters, the amplitudes of the bounded function $s_{\mu}(t)$ depend on the differences of the cluster frequencies.

In contrast to the splay states, the phase distribution fulfills $R_{2}\left(\boldsymbol{a}_{\mu}\right)=1$ for all $\mu=1,2,3$, see Fig 5.2(b), middle panel. Hence, all oscillators of a cluster have either the same phase $a_{\mu} \in[0,2 \pi)$ or the antipodal phase $a_{\mu}+\pi$ such that $2 a_{i, \mu}=2 a_{\mu}$ modulo $2 \pi$ for all $i=1, \ldots, N_{\mu}$. Therefore, the clusters represented in Fig 5.2(b) are called antipodal type clusters. Note that with this formal definition of an antipodal state, in-phase clusters belong to the class of antipodal clusters. 


\subsubsection{Mixed type cluster states}

The third type of multicluster states combines the previous two types. The 2-cluster state shown in Fig. 5.2(c) consists of one splay cluster and one antipodal cluster. We call these states mixed type multicluster. Despite using several different uniformly distributed initial conditions, we have not found multi cluster by numerical simulations. Note that uniformly distributed phases are closer to splay states than to antipodal states. In fact, due to the co-stability with splay type multiclusters, mix type multicluster states are very unlikely to find from uniformly distributed random initial conditions. In previous studies mix type multiclusters have not even been mentioned [KAS19]. In order to find these particular states, we used specially prepared initial condition motivated by the results described in Section 5.5.

As we have seen before, the interaction of a cluster with an antipodal cluster induces a modulation $s(t)$ additional to the linear growth of the oscillator's phase. In contrast, the interaction with a splay cluster does not introduce any modulation. Thus, the temporal dynamics of the oscillators in the antipodal cluster $(\mu=1)$ have $s_{i, 1}(t) \equiv 0$ while the oscillators in the splay cluster $(\mu=2)$ show additional bounded modulations $s_{i, 2}(t)$, see Fig. 5.2(c). For the oscillators of the splay cluster we plot the time series of two representatives. We notice the temporal shift in the dynamics of the two representatives of the splay cluster. The oscillators in the splay cluster are not completely temporally synchronized. More specifically we have $\phi_{i, 1}(t)=\Omega_{1} t+a_{i, 1}$ with $R_{2}\left(\boldsymbol{a}_{1}\right)=1$ for $i=1, \ldots, N_{1}$ and $\phi_{i, 2}(t)=\Omega_{2} t+a_{i, 2}+s_{i, 2}(t)$ with $R_{2}\left(\boldsymbol{a}_{2}\right)=0$ for $i=1, \ldots, N_{2}$.

Despite the complexity of the three types of multiclusters states, the structures can be broken down into simple blocks. In fact, one-cluster states of splay and antipodal type serve as building blocks in order to create more complex multicluster structures. In the following sections we provide an in-depth analysis of these blocks. 

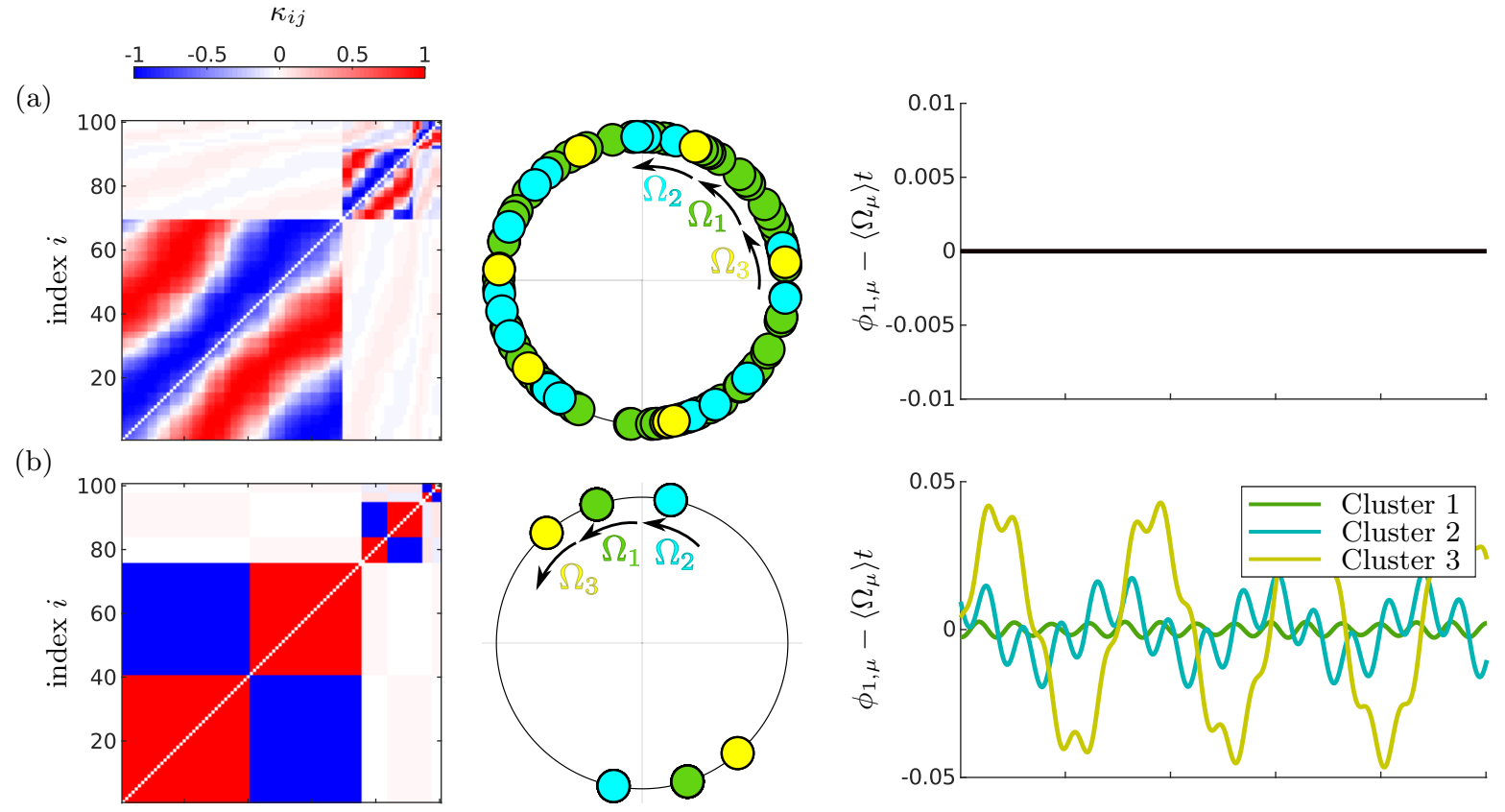

(c)
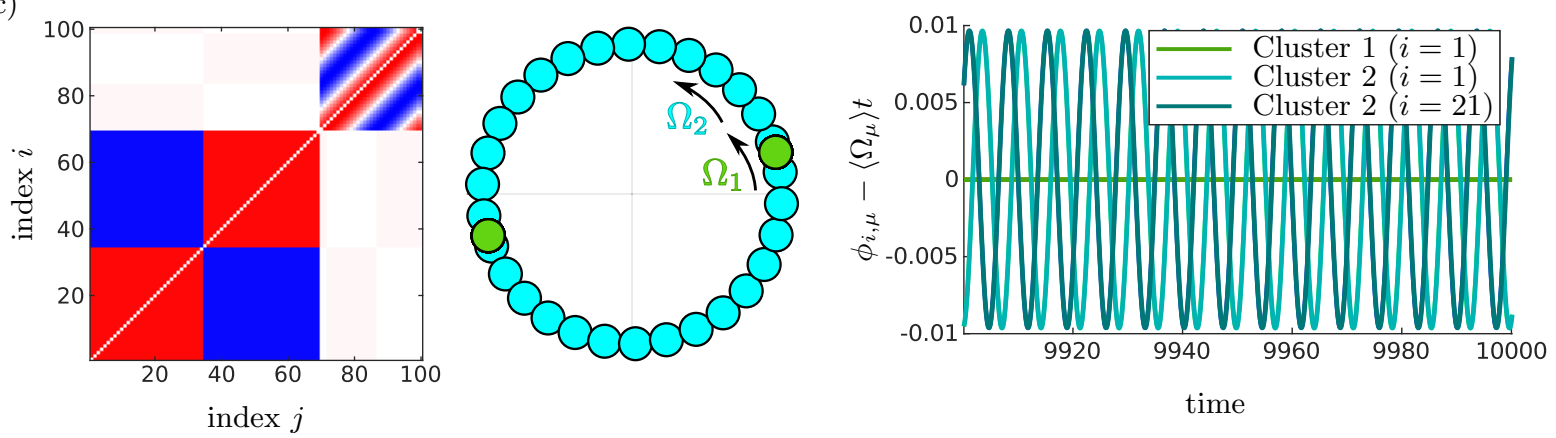

Figure 5.2: Three different types of multicluster states at $t=10000$ with $N=100$ and $\epsilon=0.01$. For all types, the coupling matrix (left), distribution of the phases (middle), and time series of representative phase oscillators from each cluster (right) are presented. In the plot of the phase distribution, each node represents one oscillator and is colored with respect to the cluster to which it belongs. The time series are shown after subtracting the average linear growth $\phi_{i, \mu}(t)-\left\langle\Omega_{\mu}\right\rangle t$. The colouring of the time series (shaded for visibility) of a representative phase oscillator from one cluster is in accordance with the pictures in the middle panel. (a) Splay type 3-cluster for $\alpha=0.3 \pi, \beta=0.23 \pi$; (b) Antipodal type 3-cluster for $\alpha=0.3 \pi, \beta=-0.53 \pi$; (c) Mixed type 2-cluster for $\alpha=0.3 \pi, \beta=-0.4 \pi$. Figure taken from [BER19a]. 


\subsection{Splay type multicluster states}

As seen in Fig. 5.2, one-cluster of splay type serve as building blocks for multicluster states. In the following, we describe this build-up explicitly.

\subsubsection{Conditions for the emergence of splay type multicluster states}

The multicluster solutions of splay type are composed by the clusters from the continuous family $S$ of phase-locked solutions with $R_{2}(\mathbf{a})=0$ and different frequencies. The following proposition describes them.

Proposition 5.2.1 System 5.1-(5.2) possesses the multicluster solution

$$
\begin{array}{cc}
\phi_{i, \mu}(t)=\Omega_{\mu} t+a_{i, \mu}, & i=1, \ldots, N_{\mu} \\
\kappa_{i j, \mu v}(t)=-\rho_{\mu \nu} \sin \left(\Delta \Omega_{\mu \nu} t+a_{i, \mu}-a_{j, \nu}+\beta-\psi_{\mu \nu}\right), & \mu 1, \ldots, M \\
& j=1, \ldots, N_{\nu} \\
& v=1, \ldots, M
\end{array}
$$

with pairwise different frequencies $\Omega_{\mu}, \Delta \Omega_{\mu \nu}:=\Omega_{\mu}-\Omega_{\nu}, \rho_{\mu \nu}:=\left(1+\left(\Delta \Omega_{\mu \nu} / \epsilon\right)^{2}\right)^{-\frac{1}{2}}$ and $\psi_{\mu \nu}:=$ $\arctan \left(\Delta \Omega_{\mu \nu} / \epsilon\right)$ if and only if

$R_{2}\left(\mathbf{a}_{\mu}\right)=0$ for all $\mu=1, \ldots, M$ and the frequencies $\left(\Omega_{1}, \ldots, \Omega_{M}\right)$ solve the following system of equations

$$
\Omega_{\mu}=\frac{1}{2 N} \sum_{\nu=1}^{M} \rho_{\mu \nu} N_{\nu} \cos \left(\alpha-\beta+\psi_{\mu \nu}\right), \quad \mu=1, \ldots, M .
$$

Note that $\rho_{\mu \nu}$ and $\psi_{\mu \nu}$ are functions of $\Omega_{\mu}-\Omega_{\nu}$.

The proof of Proposition 5.2.1 is to be found in the appendix A.3. Similarly to the one-cluster case, multicluster solutions of splay type give rise to a $(N-2 M-1)$-dimensional manifold of solutions

$$
S_{M}:=\left\{\left(\phi_{i, \mu}, \kappa_{i j, \mu \nu}\right):\left(\phi_{i, \mu}, \kappa_{i j, \mu \nu}\right) \text { as in (5.4)-(5.5), } R_{2}\left(\mathbf{a}_{\mu}\right)=0 \text { for all } \mu=1, \ldots, M\right\} .
$$

Let us remark that the collective frequencies (5.6) are only defined up to a constant due to the phase-shift symmetry of system (5.1)-(5.2) while the frequency difference is unaffected. An example of a 3-cluster solution of splay type is shown in Fig. 5.3. The solution was obtained by integrating system (5.1)- (5.2) numerically starting from random initial conditions. After sufficiently long transient time, the order of the oscillators is given by first sorting the oscillators with respect to their average frequencies. After that the oscillators with the same frequency are sorted by their phases. It can be seen from the pictures that the sizes of the three clusters $N_{\mu}$ $(\mu=1,2,3)$ possesses a hierarchical structure, i.e., $N_{3}<N_{2}<N_{1}$. The coupling strengths between oscillators of the same cluster vary in a larger range than between those of different clusters. The coupling between different clusters scales with $\epsilon$ since $\rho_{\mu \nu}=\epsilon / \Delta \Omega_{\mu \nu}+\mathcal{O}\left(\left(\epsilon / \Delta \Omega_{\mu \nu}\right)^{3}\right)$ and is 
(a)

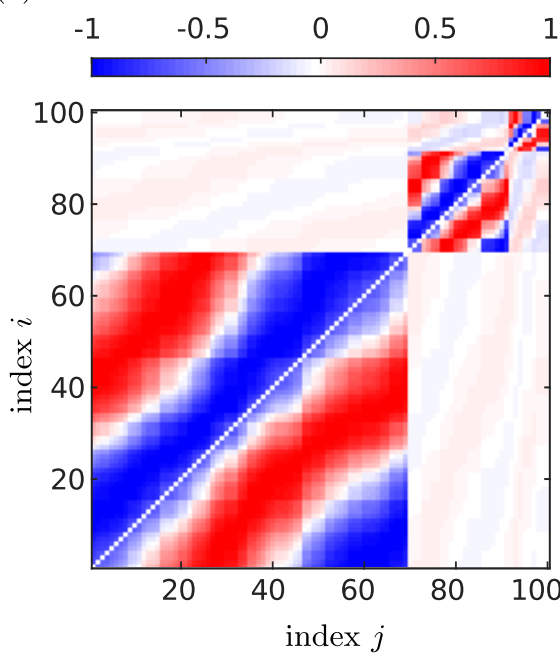

(c)

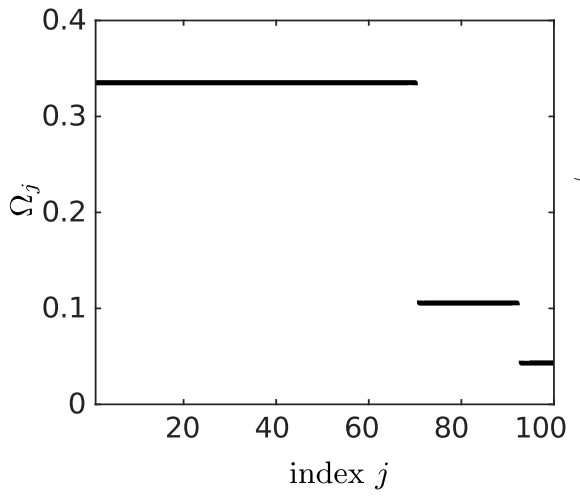

(b)
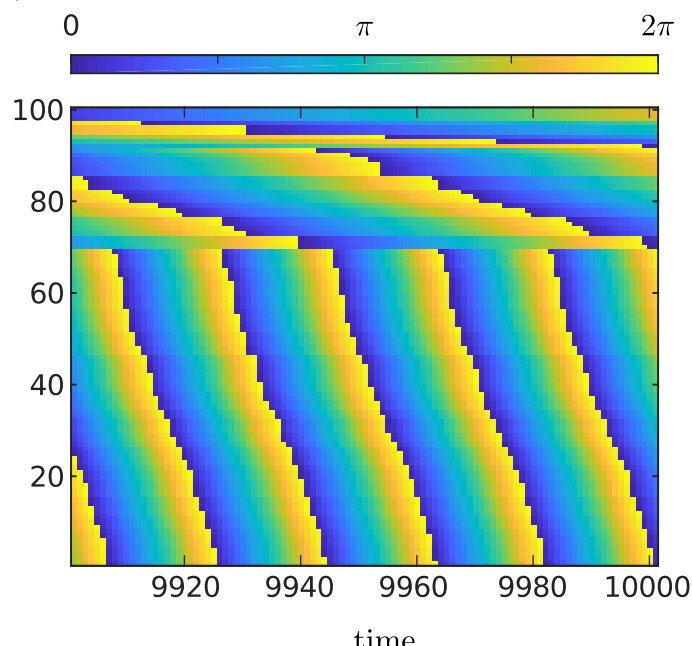

(d)

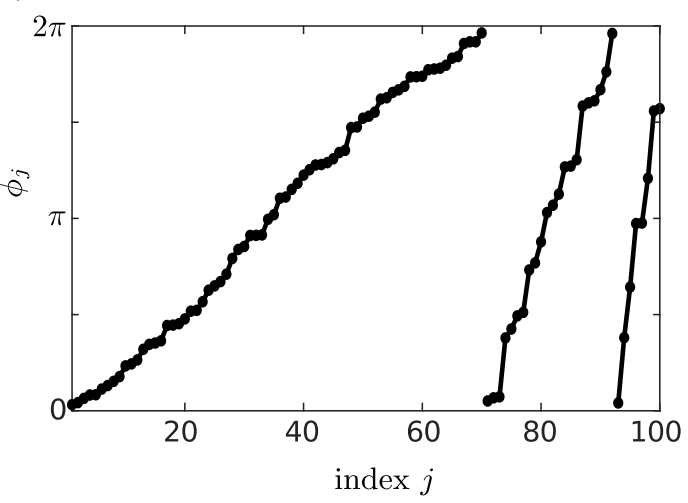

Figure 5.3: Three-cluster of splay type. (a) Coupling weights at $t=10000$ showing three clusters; (b) Distribution of the phases within each cluster; space-time raster plot; (c) Average frequency of oscillators; each plateau corresponds to one cluster; (d) Oscillator phases $\phi_{i}(t)$ at fixed time $t=10000$. Parameter values: $\epsilon=0.01, \alpha=0.3 \pi, \beta=0.23 \pi$, and $N=100$. Figure taken from [BER19].

thus close to zero (uncoupled). The oscillators of the same cluster evolve in time with the same frequencies $\dot{\phi}_{i, \mu}=\Omega_{\mu}, i=1, \ldots, N_{\mu}$.

\subsubsection{Two-cluster states of splay type}

Let us consider the case of two-clusters in more details. Let $\phi_{i, \mu}(\mu=1,2)$ with $N_{1}$ and $N_{2}$ being the numbers of oscillators in cluster 1 and 2, respectively. The following result follows from the Proposition 5.2.1.

Corollary 5.2.2 Suppose $R_{2}\left(\mathbf{a}_{\mu}\right)=0$ for $\mu=1,2$, then

$$
\begin{array}{rlrl}
\phi_{i, 1} & =\Omega_{1} t+a_{i, 1}, & i=1, \ldots, N_{1} \\
\phi_{i, 2}=\Omega_{2} t+a_{i, 2}, & i=1, \ldots, N_{2} \\
\kappa_{i j, \mu \mu}=-\sin \left(a_{i, \mu}-a_{j, \mu}+\beta\right), & \mu=1,2 \\
\kappa_{i j, \mu \nu}=-\rho_{\mu \nu} \sin \left(\Delta \Omega_{\mu \nu} t+a_{i, \mu}-a_{j, v}+\beta-\psi_{\mu \nu}\right), & \mu, v=1,2 ; \mu \neq v
\end{array}
$$




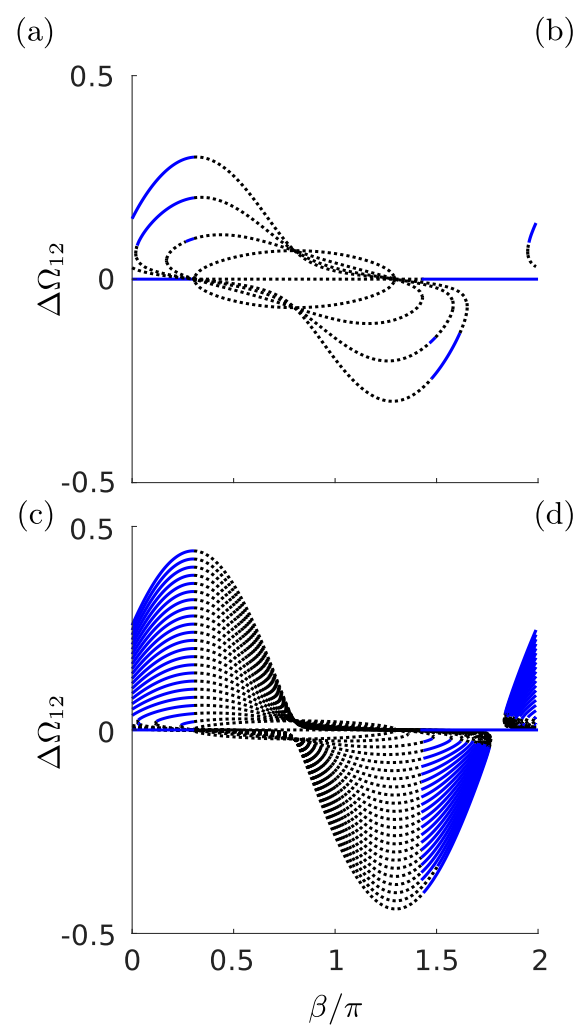

(b)

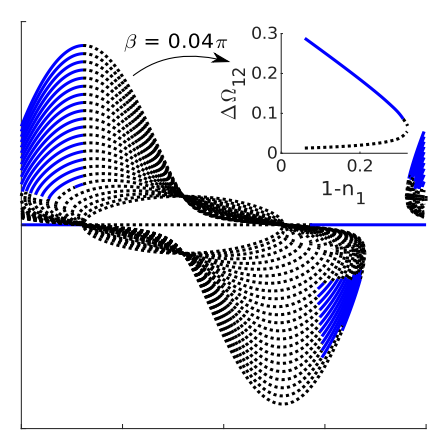

(d)

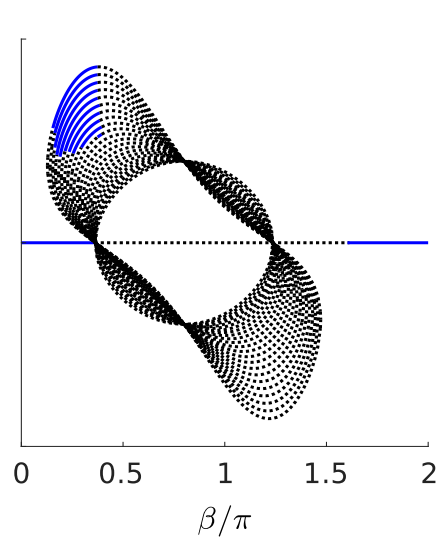

Figure 5.4: The figures show all one- and two-cluster solutions of splay type for the system (5.1)-(5.2). For this, the frequency differences $\Delta \Omega_{12}$ are displayed corresponding to the equations (4.6) and (5.7). The dotted lines (black) indicate unstable solutions while the solid lines (blue) indicate stable solutions. Here, every second solution is plotted for the sake of visibility. Parameter values: (a) $N=20, \epsilon=0.01$; (b) $N=50, \epsilon=0.01$; (c) $N=50, \epsilon=0.001$; (d) $N=50$, $\epsilon=0.1 ; \alpha=0.3 \pi$ is fixed for all panels. Figure taken from [BER19].

is a two-cluster solution of system (5.1)-(5.2) with

$$
\begin{gathered}
\left(\Delta \Omega_{12}\right)_{1,2}=\frac{1}{2}\left(n_{1}-\frac{1}{2}\right) \cos (\alpha-\beta) \pm \frac{1}{2} \sqrt{\left(n_{1}-\frac{1}{2}\right)^{2} \cos ^{2}(\alpha-\beta)-2 \epsilon(2 \epsilon+\sin (\alpha-\beta)),} \\
\Omega_{\mu}=\frac{1}{2}\left(n_{\mu} \cos (\alpha-\beta)+\rho_{\mu \nu} n_{\nu} \cos \left(\alpha-\beta+\psi_{\mu \nu}\right)\right),(\mu, v=1,2 ; \mu \neq v),
\end{gathered}
$$

where $n_{\mu}=N_{\mu} / N$ and $\psi_{\mu \nu}, \rho_{\mu \nu}$ as in Proposition 5.2.1.

The explicit expressions for the frequencies $\Delta \Omega_{12}, \Omega_{1,2}$ and other parameters of the solutions follow from the system of equations (5.6), which can be solved explicitly leading to (5.7)-(5.8) for $M=2$. For any given relative cluster size $n_{\mu}$, equations (5.7) and (5.8) provide either two, one, or no solutions corresponding to the two-cluster solution. Hence, for each fixed set of parameters, there may be up to $2(N-4)$ such solutions.

Figure 5.4 shows the frequency differences $\Delta \Omega_{12}$ of these solutions as functions of parameter $\beta$ for different number of oscillations $N$ and adaptation parameters $\epsilon$. Interestingly, the frequencies of the solutions depend only on the difference $\alpha-\beta$, see (5.7)-(5.8). By increasing the number of oscillators $N$ in the system, the number of solutions increases accordingly. This can be seen from Fig. 5.4(a-b) where we increase the number of oscillators from $N=20$ to $N=50$ with all other parameters fixed. The set of 2-cluster solutions is represented by all $\Delta \Omega_{12}(\beta)$ for a given parameter $\beta$. In accordance with (5.7) the number of solutions increases with increasing 
$N$. The region of non-existence of the multicluster solutions corresponds to the cases where the argument beneath the root in (5.7) becomes negative. The size of the existence gap depends furthermore on the choice of the time separation parameter $\epsilon$. This can be seen by comparing Fig. 5.4(b-d) where we vary the value for $\epsilon$.

\subsubsection{Adaptation rate dependence for the emergence of two-cluster states}

In this section we show the importance of the time-separation parameter $\epsilon$ for the appearance of multicluster states. In particular, we obtain the critical value $\epsilon_{c}$ above which the multicluster states cease to exist.

It is quite remarkable that in case of splay-type clusters the multicluster solution can be explicitly given as it was shown in the Proposition 5.2.1 and Corollary 5.2.2. With this, we can study directly the role of several parameters for the existence of multicluster states. In Figure 5.4(b-d), solutions for Eq. (5.7) are presented depending on the parameter $\beta$. The number of oscillators in the system is chosen as $N=50$. Each line $\Delta \Omega_{12}(\beta)$ in Fig. 5.4(b-d) represents a frequency difference of two clusters for which the two-cluster state of splay type exists with fixed relative number $n_{1}$ of oscillators in the first cluster. Note that the number of possible two-cluster states increases proportionally to the total number of oscillators $N$. Different panels show solutions for different values of $\epsilon$. We note that the existence of those two-cluster states depends only on the difference of $\gamma:=\alpha-\beta$, see Eq. (5.7). The necessary condition for the existence of a two-cluster state reads

$$
\left(n_{1}-\frac{1}{2}\right)^{2} \cos ^{2} \gamma>2 \epsilon(2 \epsilon+\sin \gamma)
$$

From Eq. (5.9) we immediately see that the value of the time separation parameter epsilon in system (5.1)-(5.2) is important for the existence of the multicluster states. This dependence is in contrast to the findings for one-cluster states. First of all, note that the left hand side of condition (5.9) is positive for $\gamma \neq \pm \pi / 2$. Hence, for all parameters, there is a critical value $\epsilon_{c}$ such that there exists no two-cluster state for $\epsilon>\epsilon_{c}$. Explicitly, we have

$$
\epsilon_{c}=-\frac{1}{4} \sin \gamma+\frac{1}{2} \sqrt{\frac{1}{4} \sin ^{2} \gamma+\left(n_{1}-\frac{1}{2}\right)^{2} \cos ^{2} \gamma},
$$

which is illustrated in Fig. 5.5. The figure shows the critical value $\epsilon_{c}$ depending on the parameter $\gamma$ for different values of $n_{1}$. The function possesses a global maximum with $\epsilon_{c}=0.5$. This means that there is a particular requirement on the time separation in order to have two-cluster states of splay type. Indeed, the adaptation of the network has to be at most half as fast as the dynamics of the oscillatory system.

Further let us remark that the two-cluster state with equally sized clusters $n_{1}=0.5$ exists only for $\alpha-\beta \in(\pi, 2 \pi)$, i.e., $\epsilon_{c}=0$ for all $\alpha-\beta \in[0, \pi]$.

In the subsequent sections we discus that the combination of one-cluster states to a multicluster state can result in modulated dynamics of the oscillators additional to the linear growth. In fact, this additional temporal behavior is due to the interaction of the clusters. As we see in Fig. 5.2(a), 


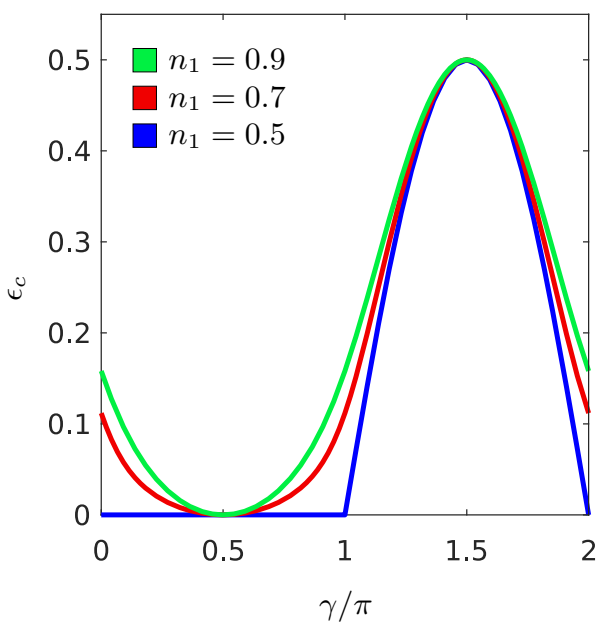

Figure 5.5: For the case of two-cluster states of splay type, the critical value $\epsilon_{c}$ of time-separation parameter $\epsilon$ is plotted as a function of $\gamma=\alpha-\beta$ for different cluster sizes $n_{1}=N_{1} / N$. The function is given explicitly by Eq. (5.10). Figure taken from [BER19a].

oscillators interacting with a splay-type cluster will not be forced to perform additional dynamics. This is the reason why we are able to derive a closed analytic expression for multicluster states of splay type. It is possible to determine the frequencies explicitly as in Eq. (5.7). Therefore, here the interaction between cluster causes only changes in the collective frequencies which are small whenever $\epsilon$ is small.

In contrast to splay clusters, the interaction with antipodal cluster leads to bounded modulation of the oscillator dynamics besides the constant-frequency motion. The modulations scale with $\epsilon$ and hence depend on the time-separation parameter. In case of a mixed type two-cluster state, both interaction phenomena are present. The oscillators in the antipodal cluster interact with the splay cluster leading to no additional modulation. On the contrary, the phase oscillators of the splay cluster get additional modulation via the interaction with the antipodal cluster.

In the following Section 5.3, we provide a general result on the asymptotic existence of splay, antipodal, and mixed type multicluster states. In the Section 5.4 and 5.5, this general result will be applied to give explicit expression for antipodal as well as mixed type multiclusters.

\subsection{Conditions for the emergence of multicluster states - A generalized approach}

In this section we give an analytic description of multicluster solutions in terms of an asymptotic expansion. We consider therefore the expansion of $r$-th order together with a multi-time scale ansatz [VER06]

$$
\begin{array}{rr}
\phi_{i, \mu}^{(r)}(\epsilon, t):=\Omega_{\mu}^{(r)}\left(\tau_{0}, \ldots, \tau_{r}\right)+a_{i, \mu}+\sum_{l=1}^{r} \epsilon^{l} p_{i, \mu ; l}(t) & \mu, v=1, \ldots, M \\
\kappa_{i j, \mu \nu}^{(r)}(\epsilon, t):=\sum_{l=0}^{r} \epsilon^{l} k_{i j, \mu \nu ; l}(t) & i, j=1, \ldots, N_{\mu}
\end{array}
$$


where $\Omega_{\mu}^{(r)} \in C^{1}\left(\mathbb{R}^{r+1}\right)$ is a function depending on the multi-time scales $\tau_{l}:=\epsilon^{l} t$. We show under under which conditions this expansion describes the time evolution for the system (5.1)-(5.2). The main result on the asymptotic expansion for (pseudo) multicluster solutions reads as follows.

Proposition 5.3.1 Let $r \in \mathbb{N}$. Suppose the system (5.1)- (5.2) possesses a (pseudo) multicluster solution $\left(\phi_{i, \mu}, \kappa_{i j, \mu \nu}\right)$ with $\phi_{i, \mu}(\epsilon, t)=\Omega_{\mu}(\epsilon) t+a_{i, \mu}+s_{i, \mu}(\epsilon, t)$ where $a_{i, \mu} \in \mathbb{T}^{1}$ and the coupling matrix $\kappa_{i j, \mu v}(\epsilon, t)$ is given as the parametrization of the pullback attractor defined in (A.14). Assume further that $M_{1}$ clusters are of antipodal type $\left(2 a_{i, \mu}=a_{\mu}\right)$ and $M_{2}$ are of splay type $\left(R_{2}\left(\mathbf{a}_{\mu}\right)=0\right)$. Then, the $r$-th order asymptotic expansion of $\phi_{i, \mu}(\epsilon, t)$ for $t \in O\left(1 / \epsilon^{r}\right)$ as $\epsilon \rightarrow 0$ is given by

$$
\begin{array}{rlrl}
\phi_{i, \mu}^{(r)}(\epsilon, t) & :=\Omega_{\mu, 0}^{(r)} t+a_{i, \mu}+\sum_{l=1}^{r} \epsilon^{l}\left(\Omega_{\mu, l}^{(r)} t+p_{i, \mu ; l}(t)\right) & \mu, v & =1, \ldots, M \\
\kappa_{i j, \mu \nu}^{(r)}(\epsilon, t) & :=\sum_{l=0}^{r} \epsilon^{l} k_{i j, \mu v ; l}(t), & i & =1, \ldots, N_{\mu} \\
& j & =1, \ldots, N_{\nu}
\end{array}
$$

where

(i) all coefficients of the expansion can be found inductively;

(ii) the first order approximation can be written as

$$
\phi_{i, \mu}^{(1)}=\left(\frac{n_{\mu}}{2}(\cos (\alpha-\beta)-\cos (\alpha+\beta))-\epsilon \sum_{\nu=M_{1}+1}^{M} \frac{n_{v}}{2 \Delta \Omega_{\mu \nu}^{(1)}} \sin (\alpha-\beta)\right) t+a_{i, \mu}+\epsilon p_{\mu ; 1}
$$

for $\mu=1, \ldots, M_{1}$, and

$$
\phi_{i, \mu}^{(1)}=\left(\frac{n_{\mu}}{2} \cos (\alpha-\beta)-\epsilon \sum_{\substack{\nu=M_{1}+1 \\ \nu \neq \mu}}^{M} \frac{n_{\nu}}{2 \Delta \Omega_{\mu \nu}^{(1)}} \sin (\alpha-\beta)\right) t+a_{i, \mu}+\epsilon p_{i, \mu ; 1}(t)
$$

for $\mu=M_{1}+1, \ldots, M$ with

$$
\begin{array}{rr}
p_{\mu ; 1}=-\sum_{\substack{\nu=1 \\
\nu \neq \mu}}^{M_{1}} \frac{n_{\nu}}{4\left(\Delta \Omega_{\mu \nu}^{(1)}\right)^{2}} \cos \left(2 \Delta \Omega_{\mu \nu}^{(1)} t+a_{\mu}-a_{\nu}+\alpha+\beta\right) & \mu=1, \ldots, M_{1} \\
p_{i, \mu ; 1}=-\sum_{\nu=1}^{M_{1}} \frac{n_{\nu}}{4\left(\Delta \Omega_{\mu \nu}^{(1)}\right)^{2}} \cos \left(2 \Delta \Omega_{\mu \nu}^{(1)} t+2 a_{i, \mu}-a_{\nu}+\alpha+\beta\right) ; & \mu=M_{1}+1, \ldots, M
\end{array}
$$

the coupling weights are given by

$$
\begin{aligned}
\kappa_{i j, \mu \mu}^{(1)} & =-\sin \left(a_{i, \mu}-a_{j, \mu}+\beta\right), \\
\kappa_{i j, \mu \nu}^{(1)} & =\frac{\epsilon}{\Delta \Omega_{\mu \nu}^{(1)}} \cos \left(\Delta \Omega_{\mu \nu}^{(1)} t+a_{i, \mu}-a_{j, \nu}+\beta\right) ;
\end{aligned}
$$


and the cluster frequencies $\Omega_{\mu}^{(1)}$ solve the system of equations

$$
\Omega_{\mu}^{(1)}=\left(\Omega_{\mu ; 0}-\epsilon \sum_{\substack{\nu=1 \\ \nu \neq \mu}}^{M} \frac{n_{\nu}}{2 \Delta \Omega_{\mu \nu}^{(1)}} \sin (\alpha-\beta)\right)
$$

with

$$
\begin{array}{lr}
\Omega_{\mu ; 0}=n_{\mu} \sin (\alpha) \sin (\beta) & \mu=1, \ldots, M_{1} \\
\Omega_{\mu ; 0}=\frac{n_{\mu}}{2} \cos (\alpha-\beta) . & \mu=M_{1}+1, \ldots, M
\end{array}
$$

where $\mu=1, \ldots, M, i, j=1, \ldots, N_{\mu}$ and $\Delta \Omega_{\mu \nu}^{(1)}:=\Omega_{\mu}^{(1)}-\Omega_{\nu}^{(1)}$.

The proof makes use of several lemmas and is presented in the appendix A.4. Overall, we aim to describe the following particular form for the dynamical behaviour of the phase oscillators. The phases of the oscillators $\phi_{i, \mu}$ form a pseudo multicluster, c.f. definition 5.5.1. Further, the bounded modulations for the phases of each oscillator are given as Taylor expansions in $\epsilon$ with periodic coefficients that can be expressed as Fourier sums with even modes. The strategy for the proof of the main result is as follows.

1. Assume that the phases of the oscillators are given as finite Taylor sums in $\epsilon$ with periodic coefficients, which are represented as finite Fourier sums. With this, the equations (5.2) can be explicitly solved. Introducing the pull-back attractor provides us with a unique expression for the asymptotic solutions $(t \rightarrow \infty)$. An explicit form for the expansion in $\epsilon$ of these solutions of the coupling weights $\kappa$ is given in Lemma A.4.2.

2. The solutions of the coupling weights depend on the Fourier modes of the periodic expansion coefficients of the oscillators. An statement on their explicit dependence is provided by Lemma A.4.3. More specifically, the expansion coefficients of the couplings weights only consist of even modes whenever the expansion coefficients for the phases of the oscillators consist only of odd modes.

3. The expressions for the coupling weights in Lemma A.4.2 are used to derive the explicit form for the phase oscillators. More specifically, the expansions coefficients are derived such that they satisfy the equations (5.1). Higher order terms which contribute to a linear growth are absorbed in an expansion for the oscillator frequencies.

4. Finally, we find an iterative scheme to determine all expansion coefficients of the phases and coupling weights up to any order. Moreover, it is shown that the coefficients provided by the iterative scheme are consistent with the assumption on the expansion coefficients given in the beginning of the proof.

In the following this general result is applied to antipodal and mixed multicluster in order to characterize their properties. Note that if we apply the expansion result to splay type multicluster, i.e. $M_{1}=0$, the expressions would agree with the Taylor expansion in $\epsilon$ of the explicit formulas presented in Proposition 5.2.1. 


\subsection{Antipodal type multicluster states}

In this section we apply the results presented in Proposition 5.2.1 to antipodal type multicluster states.

\subsubsection{Asymptotic conditions for the emergence of antipodal type multicluster states}

In the case when the oscillators are phase synchronized or in an antiphase relation within each cluster, the situation is different to what was described for the splay type multiclusters. Particularly, the linear growth of the oscillator phases within each cluster is modulated by periodic or quasi-periodic terms of order $\epsilon$. That is, the clusters possess the form $\phi_{i, \mu}(t)=\Omega_{\mu} t+$ $a_{i, \mu}+\epsilon p_{\mu}(t, \epsilon)$. Here, we give important necessary conditions for the existence of such solutions and their asymptotic expansion in $\epsilon$. Additionally, we provide numerical results showing these solutions. In particular, we present a system of equations for the cluster frequencies $\Omega_{\mu}$.

Proposition 5.4.1 Suppose $2 a_{i, \mu}=a_{\mu} \bmod 2 \pi$ for all $\mu=1, \ldots, M$ and $i=1, \ldots, N_{\mu}$. If system (5.1)-(5.2) possesses antipodal multicluster solution $\left(\phi_{i, \mu}, \kappa_{i j, \mu \nu}\right)$ then its first asymptotic expansion in $\epsilon$ is given by

$$
\begin{aligned}
\phi_{i, \mu}^{(1)} & =\Omega_{\mu}^{(1)} t+a_{i, \mu}-\epsilon \sum_{\substack{\nu=1 \\
\nu \neq \mu}}^{M} \frac{n_{\nu}}{4\left(\Delta \Omega_{\mu \nu}^{(1)}\right)^{2}} \cos \left(2 \Delta \Omega_{\mu \nu}^{(1)} t+a_{\mu}-a_{\nu}+\alpha+\beta\right), \\
\kappa_{i j, \mu \mu}^{(1)} & =-\sin \left(a_{i, \mu}-a_{j, \mu}+\beta\right), \\
\kappa_{i j, \mu \nu}^{(1)} & =\frac{\epsilon}{\Delta \Omega_{\mu \nu}^{(1)}} \cos \left(\Delta \Omega_{\mu \nu}^{(1)} t+a_{i, \mu}-a_{j, \nu}+\beta\right), \quad \mu \neq v
\end{aligned}
$$

with the cluster frequencies $\Omega_{\mu}^{(1)}$ up to first order in $\epsilon$ whenever the following implicit equation can be solved

$$
\Omega_{\mu}^{(1)}=\left(n_{\mu} \sin (\alpha) \sin (\beta)-\epsilon \sum_{\substack{\nu=1 \\ \nu \neq \mu}}^{M} \frac{n_{\nu}}{2 \Delta \Omega_{\mu \nu}^{(1)}} \sin (\alpha-\beta)\right) .
$$

Here, $\mu=1, \ldots, M, i, j=1, \ldots, N_{\mu}$ and $\Delta \Omega_{\mu v}:=\Omega_{\mu}^{(1)}-\Omega_{v}^{(1)}$.

This first order perturbation reveals a nonlinear modulation $p_{\mu}$, which is periodic or quasiperiodic with the frequencies $\Delta \Omega_{\mu \nu}^{(1)}$ given by the differences in the frequencies of the clusters.

Figure 5.6 shows the numerically obtained 3-cluster solution of antipodal type. The dynamics of system (5.1)-(5.2) is shown after a sufficiently long transient so that it represents dynamically stable solution (more on stability in Section 5.6). One can clearly observe three clusters in the coupling matrix. Similarly to the previous multicluster cases, we first sort the oscillators with respect to their average frequency and subsequently by their phases. In contrast to the splayed distribution of the phases described in Section 5.2, the oscillators within the clusters additionally form two groups, in which the phases differ by $\pi$. 


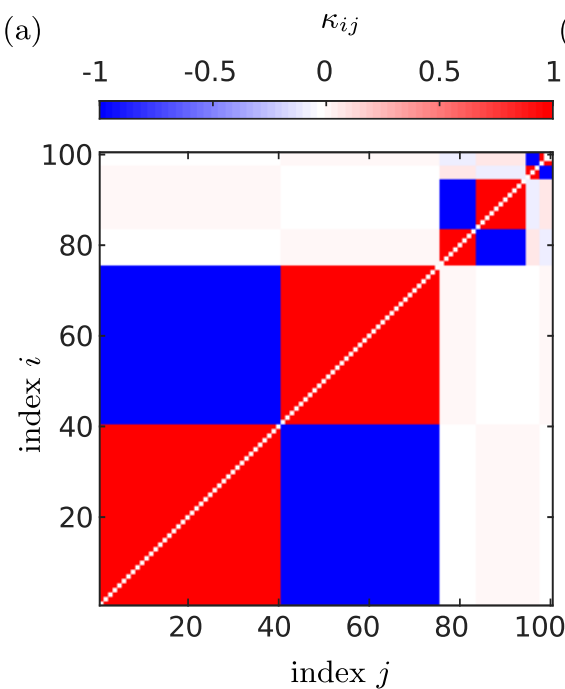

(b) $\quad \phi_{i}$

(c)
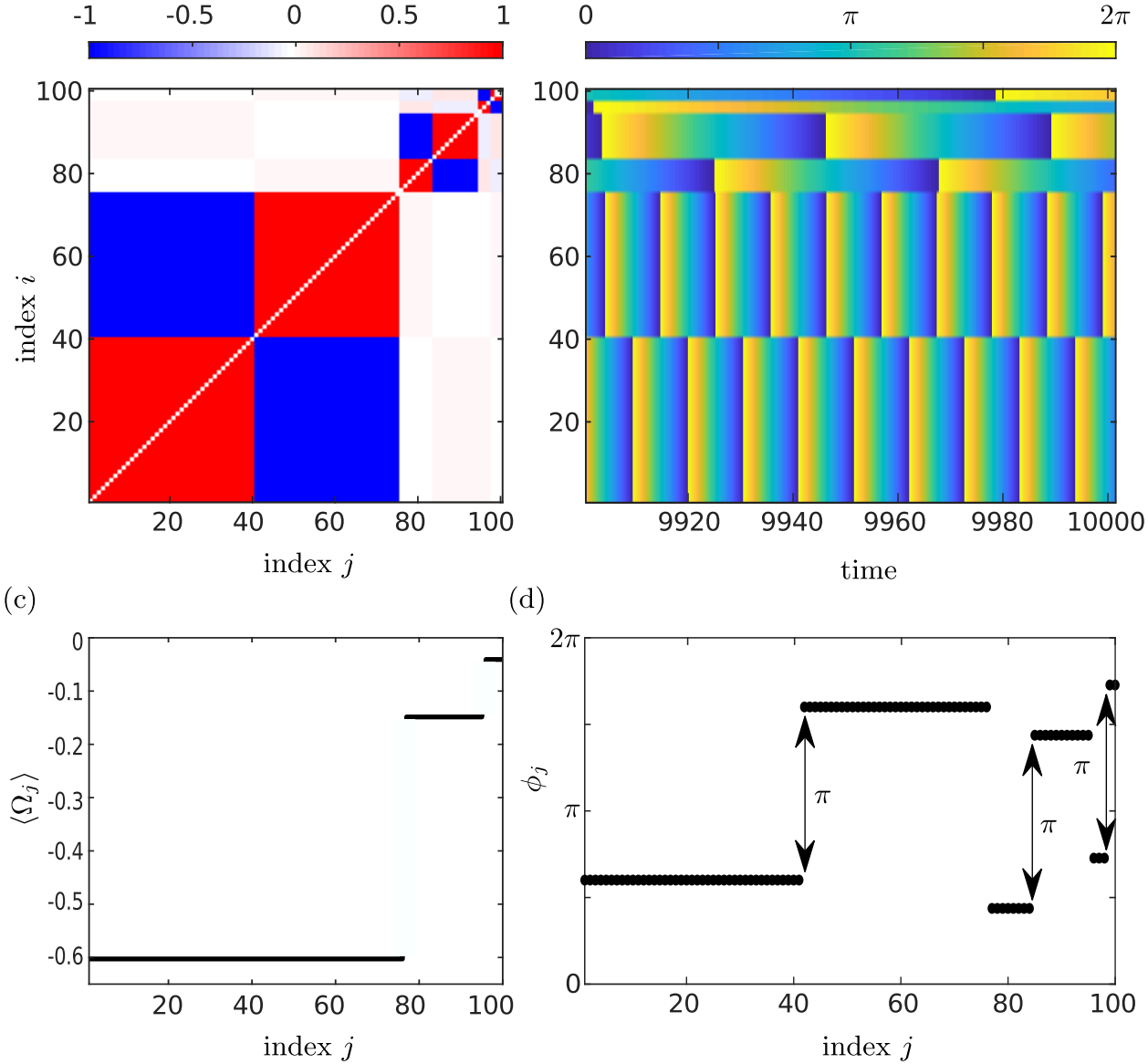

(d)

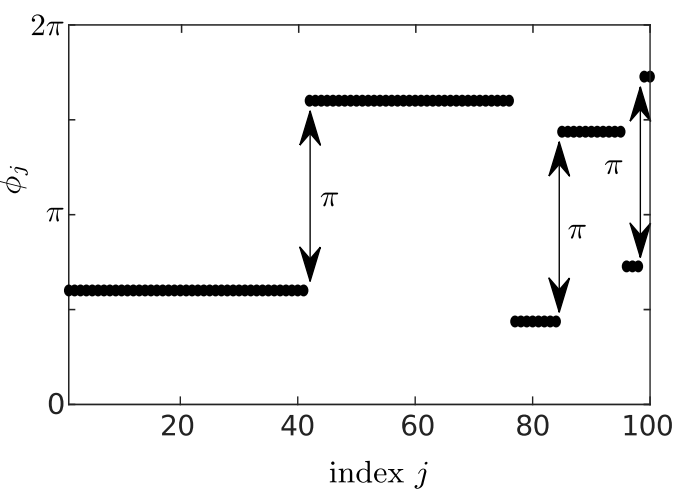

Figure 5.6: Three-cluster of antipodal type. (a) Coupling weights at $t=10000$ showing three clusters; (b) Distribution of the phases within each cluster; space-time raster plot; (c) Average frequency of oscillators; each plateau corresponds to one cluster; (d) Oscillator phases $\phi_{i}(t)$ at fixed time $t=10000$. Parameter values: $\epsilon=0.01, \alpha=0.3 \pi, \beta=-0.53 \pi$, and $N=100$. Figure taken from [BER19].

(a)

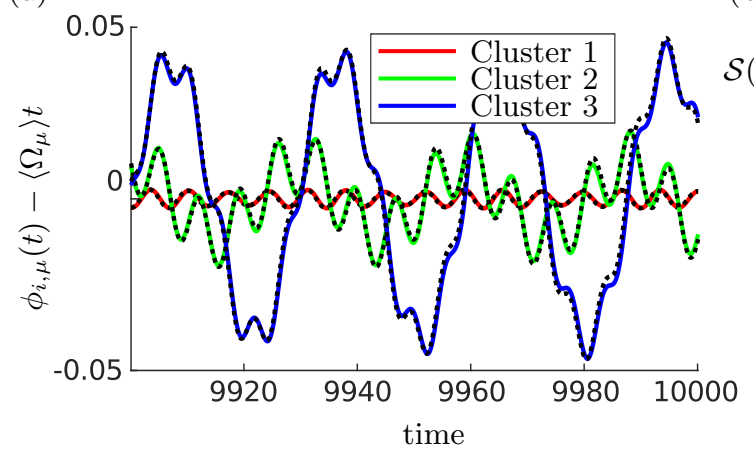

(b)

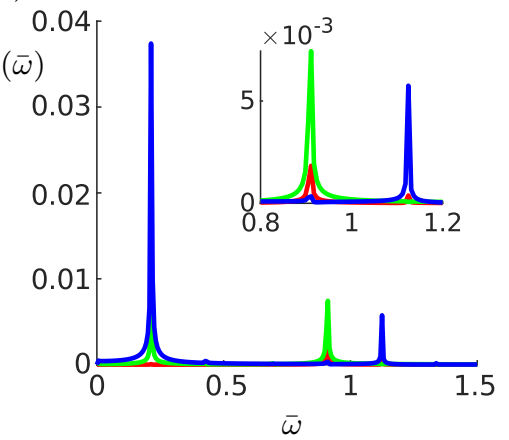

Figure 5.7: For 3-cluster solution from Fig. 5.6, panel (a) shows time series of an oscillator from one of the clusters after subtracting the average linear growth $\phi_{\mu, i}(t)-\left\langle\Omega_{\mu}\right\rangle t$. The black dashed lines show the corresponding analytic results from the asymptotic expansion in Proposition 5.4.1. (b) Power spectrum of the time series given in (a). Figure modified from [BER19]. 
In order to observe the modulation of the cluster frequencies, time series for three representative oscillators from each cluster are shown in Fig. 5.7(a). The averaged linear growth of the phases due to $\left\langle\Omega_{\mu}\right\rangle t$ has been subtracted to show the modulation. Such small but non-vanishing oscillations do not exist in the case of splay type multiclusters. In addition, the black dashed lines show the modulation given by Proposition 5.4.1 confirming that the asymptotic expansion describes the whole temporal behaviour very well. Furthermore, Proposition 5.4.1 implies that the amplitudes of the modulations are proportional to $n_{v} /\left(\Delta \Omega_{\mu \nu}^{(1)}\right)^{2}$. Thus, if the difference in the frequencies is high the amplitude is small and vice versa. This relation is also reflected by the power spectrum, see Fig. 5.7(b). Figure 5.7(b) confirms that the frequencies of the modulation oscillations correspond to the differences of the average frequencies.

\subsubsection{Two-cluster states of antipodal type}

Let us consider the case of two clusters in more details. Let $N_{1}$ and $N_{2}=N-N_{1}$ be the numbers of oscillators in group 1 and 2, respectively. The following result follows from Proposition 5.4.1.

Corollary 5.4.2 Suppose $2 a_{i, \mu}=a_{\mu}$ for all $\mu=1,2$ and $i=1, \ldots, N_{\mu}$. If system (5.1)-(5.2) possesses an antipodal multicluster solution $\left(\phi_{i, \mu}, \kappa_{i j, \mu \nu}\right)$ then its first order asymptotic expansion in $\epsilon$ is given by

$$
\begin{aligned}
\phi_{i, 1}^{(1)} & =\Omega_{1}^{(1)} t+a_{i, 1}-\epsilon \frac{n_{2}}{4\left(\Delta \Omega_{12}^{(1)}\right)^{2}} \cos \left(2 \Delta \Omega_{12}^{(1)} t+a_{1}-a_{2}+\alpha+\beta\right), \\
\phi_{i, 2}^{(1)} & =\Omega_{2}^{(1)} t+a_{i, 2}-\epsilon \frac{n_{1}}{4\left(\Delta \Omega_{12}^{(1)}\right)^{2}} \cos \left(2 \Delta \Omega_{12}^{(1)} t+a_{1}-a_{2}-\alpha-\beta\right), \\
\kappa_{i j, \mu \mu}^{(1)} & =-\sin \left(a_{i, \mu}-a_{j, \mu}+\beta\right), \\
\kappa_{i j, \mu \nu}^{(1)} & =\frac{\epsilon}{\Delta \Omega_{\mu \nu}^{(1)}} \cos \left(\Delta \Omega_{\mu \nu}^{(1)} t+a_{i, \mu}-a_{j, \nu}+\beta\right),
\end{aligned}
$$

with

$$
\Omega_{\mu}^{(1)}=\left(n_{\mu} \sin (\alpha) \sin (\beta)-\epsilon \frac{n_{\nu}}{2 \Delta \Omega_{\mu \nu}^{(1)}} \sin (\alpha-\beta)\right)
$$

for $\mu=1,2, v \neq \mu, i, j=1, \ldots, N_{\mu}, \Delta \Omega_{\mu \nu}^{(1)}:=\Omega_{\mu}^{(1)}-\Omega_{\nu}^{(1)}$,

$$
\left(\Delta \Omega_{12}^{(1)}\right)_{1,2}=\left(n_{1}-\frac{1}{2}\right) \sin (\alpha) \sin (\beta) \pm \sqrt{\left(n_{1}-\frac{1}{2}\right)^{2} \sin ^{2} \alpha \sin ^{2} \beta-\frac{\epsilon}{2} \sin (\alpha-\beta)} .
$$

This result follows directly from Proposition 5.4.1. It shows, in particular, that the system of equations (5.14) can be solved explicitly by (5.15) in case of two clusters.

Similarly to the splay multiclusters, for any fixed set of parameters and each $n_{1}$, equation (5.15) can lead to two antipodal multiclusters with two different frequency differences. Hence, a large number of antipodal two-clusters can coexist for the same parameter values. Figure 5.8 illustrates such a coexistence, where we present the one-cluster solutions given by (4.6) and 

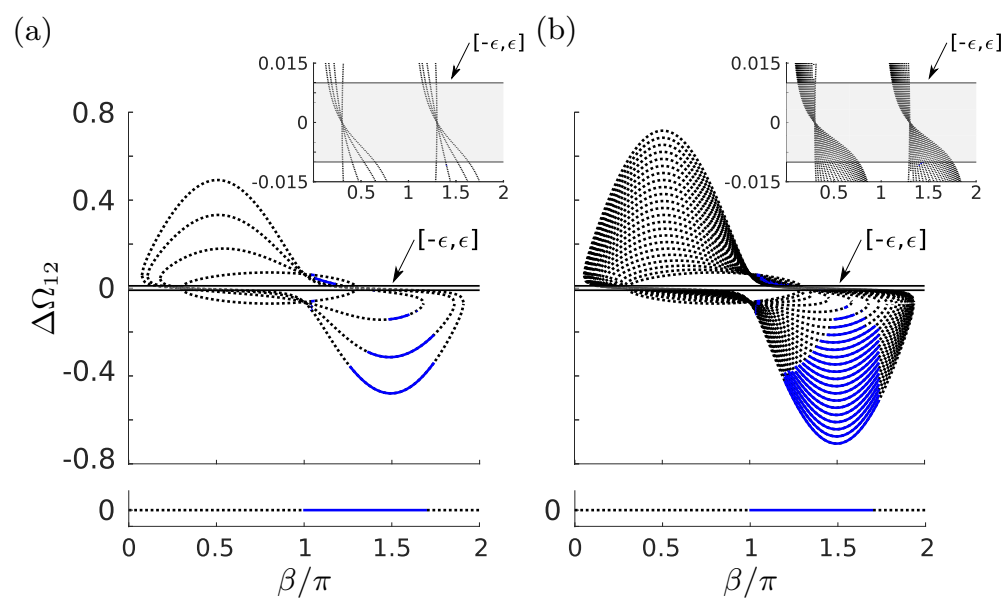

Figure 5.8: Two-cluster solutions (upper panels) and one-cluster solutions (lower panels) of antipodal type given by the asymptotic expansion in Corollary 5.4.2 and Proposition 4.1.1, respectively. For this, the difference of the frequencies $\Delta \Omega_{12}^{(1)}$ is displayed corresponding to (5.15) and (4.6). The dotted lines (black) indicate unstable solutions while the solid lines (blue) indicate stable solutions. Here, every second solution is plotted for the sake of visibility. The insets show a blow-up of the interval $[-\epsilon, \epsilon]$. Parameter values: (a) $N=20, \epsilon=0.01$; (b) $N=50, \epsilon=0.01 ; \alpha=0.3 \pi$ is fixed for all panels. Figure taken from [BER19].

the solutions to the equation (5.15). Blue solid lines represent those solutions for which the asymptotic expansion led to an existing and stable two-cluster solutions of antipodal type. Note further that for two-cluster solutions of antipodal type, the asymptotic expansion presented in Proposition 5.4.1 turns into a formal expansion whenever $|\Delta \Omega|>\epsilon$, i.e., $\epsilon$ is not assumed to be infinitesimal $(\epsilon \rightarrow 0)$. The interval $[-\epsilon, \epsilon]$ is therefore highlighted in Fig. 5.8.

\subsection{Mixed type pseudo-multicluster states}

In this section we apply the results presented in Proposition 5.2.1 to mixed type multicluster states. In order to this the notion of multiclusters has to be slightly adjusted which leads to the definition of pseudo multicluster states.

\subsubsection{Asymptotic conditions for the emergence of mixed type pseudo-multicluster states}

We have seen how clusters are described consisting of oscillator groups of splay type (Section 5.2) as well as clusters consisting of oscillator groups with in- and anti-phase relation (Section 5.4). It is therefore reasonable to ask for multicluster solutions that consist of both of these types. In order to describe these solutions we have to loosen the definition of a multicluster solution.

Definition 5.5.1 Phase oscillators $\phi_{i}(t)$ form a pseudo multicluster if they can be separated into $M$ groups such that for all $\mu \in\{1, \ldots, M\}$ the phase oscillators $\phi_{i, \mu}, i \in\left\{1, \ldots, N_{\mu}\right\}$, from each group $\mu$ satisfy $\phi_{i, \mu}(t)=\Omega_{\mu} t+s_{i, \mu}(t)$ with bounded functions $s_{i, \mu}$.

Note that every multicluster solution is by definition already a pseudo multicluster solution. 
Proposition 5.5.1 Suppose $2 a_{i, \mu}=a_{\mu}$ for all $\mu=1, \ldots, M_{1}$, and $R_{2}\left(\mathbf{a}_{\mu}\right)=0$ for all $\mu=M_{1}+1, \ldots, M$, $i=1, \ldots, N_{\mu}$ where $M_{1}$ is the number of antipodal type clusters. The mixed pseudo multicluster solutions of (5.1)-(5.2) with $\phi_{i, \mu}(t)=\Omega_{\mu}(\epsilon) t+s_{i, \mu}(t)+a_{i, \mu}$ possess the following first order asymptotic expansion in $\epsilon$

$$
\begin{aligned}
\phi_{i, \mu}^{(1)} & =\Omega_{\mu}^{(1)} t+a_{i, \mu}+\epsilon p_{i, \mu ; 1}(t), \\
\kappa_{i j, \mu \mu}^{(1)} & =-\sin \left(a_{i, \mu}-a_{j, \mu}+\beta\right), \\
\kappa_{i j, \mu \nu}^{(1)} & =\frac{\epsilon}{\Delta \Omega_{\mu \nu}^{(1)}} \cos \left(\Delta \Omega_{\mu \nu}^{(1)} t+a_{i, \mu}-a_{j, \nu}+\beta\right),
\end{aligned}
$$

with

$$
p_{i, \mu ; 1}(t)=p_{\mu ; 1}=-\sum_{\substack{\nu=1 \\ \nu \neq \mu}}^{M_{1}} \frac{n_{\nu}}{4\left(\Delta \Omega_{\mu \nu}^{(1)}\right)^{2}} \cos \left(2 \Delta \Omega_{\mu \nu}^{(1)} t+a_{\mu}-a_{\nu}+\alpha+\beta\right)
$$

for $\mu=1, \ldots, M_{1}$,

$$
p_{i, \mu ; 1}(t)=-\sum_{\substack{\nu=1 \\ \nu \neq \mu}}^{M} \frac{n_{\nu}}{4\left(\Delta \Omega_{\mu \nu}^{(1)}\right)^{2}} \cos \left(2 \Delta \Omega_{\mu \nu}^{(1)} t+a_{i, \mu}-a_{\nu}+\alpha+\beta\right)
$$

for $\mu=M_{1}+1, \ldots, M$, and the cluster frequencies $\Omega_{\mu}^{(1)}$ up to second order in $\epsilon$ whenever the following system of equations can be solved

$$
\Omega_{\mu}^{(1)}=\left(\Omega_{\mu ; 0}-\epsilon \sum_{\substack{v=1 \\ \nu \neq \mu}}^{M} \frac{n_{\nu}}{2 \Delta \Omega_{\mu \nu}^{(1)}} \sin (\alpha-\beta)\right)
$$

with

$$
\begin{array}{lr}
\Omega_{\mu ; 0}=n_{\mu} \sin (\alpha) \sin (\beta) & \mu=1, \ldots, M_{1} \\
\Omega_{\mu ; 0}=\frac{n_{\mu}}{2} \cos (\alpha-\beta) . & \mu=M_{1}+1, \ldots, M
\end{array}
$$

Here, $\mu=1, \ldots, M, i, j=1, \ldots, N_{\mu}$ and $\Delta \Omega_{\mu \nu}^{(1)}:=\Omega_{\mu}^{(1)}-\Omega_{\nu}^{(1)}$.

\subsubsection{Pseudo-two-cluster states of mixed type}

As in the previous sections, we are going to show that equation (5.16) possesses solutions. For this, we consider the case of two clusters $\phi_{i, \mu}(\mu=1,2)$.

Corollary 5.5.2 Suppose $2 a_{i, 1}=a_{1}$ for all $i=1, \ldots, N_{1}$ and $R\left(\mathbf{a}_{2}\right)=0$. The mixed pseudo multiclusters 
of system (5.1)-(5.2) possess the following first order asymptotic expansion in $\epsilon$

$$
\begin{aligned}
\phi_{i, 1}^{(1)} & =\Omega_{1}^{(1)} t+a_{i, 1}, \\
\phi_{i, 2}^{(1)} & =\Omega_{2}^{(1)} t+a_{i, 2}-\epsilon \frac{n_{1}}{4\left(\Delta \Omega_{12}^{(1)}\right)^{2}} \cos \left(2 \Delta \Omega_{12}^{(1)} t+a_{1}-a_{i, 2}-\alpha-\beta\right), \\
\kappa_{i j, \mu \mu}^{(1)} & =-\sin \left(a_{i, \mu}-a_{j, \mu}+\beta\right), \\
\kappa_{i j, \mu \nu}^{(1)} & =\frac{\epsilon}{\Delta \Omega_{\mu \nu}^{(1)}} \cos \left(\Delta \Omega_{\mu \nu}^{(1)} t+a_{i, \mu}-a_{j, \nu}+\beta\right),
\end{aligned}
$$

where

$$
\begin{gathered}
\Omega_{\mu}^{(1)}=\left(\Omega_{\mu ; 0}-\epsilon \frac{n_{\nu}}{2 \Delta \Omega_{\mu \nu}^{(1)}} \sin (\alpha-\beta)\right), \\
\Omega_{1 ; 0}=n_{1} \sin (\alpha) \sin (\beta), \\
\Omega_{2 ; 0}=\frac{n_{2}}{2} \cos (\alpha-\beta), \\
\left(\Delta \Omega_{12}^{(1)}\right)_{1,2}=\frac{1}{2}\left[\left(n_{1}-\frac{1}{2}\right) \cos (\alpha-\beta)-\frac{n_{1}}{2} \cos (\alpha+\beta)\right] \\
\pm \frac{1}{2} \sqrt{\left[\left(n_{1}-\frac{1}{2}\right) \cos (\alpha-\beta)-\frac{n_{1}}{2} \cos (\alpha+\beta)\right]^{2}-2 \epsilon \sin (\alpha-\beta)}
\end{gathered}
$$

for $\mu=1,2, v \neq \mu$ and $i, j=1, \ldots, N_{\mu}$.

Illustration of the mixed 2-clusters is shown in Fig. 5.9.

Moreover, we performed a Fourier analysis of the temporal behaviour of the oscillators, see Fig. 5.10. First, it can be observed that the oscillators representing the second cluster $(i=$ $10,30)$ show the same evolution in time but with a phase lag due to the spatial dependency described above. In order to show the agreement with the asymptotic expansion presented in Corollary 5.5.2, the analytic results are displayed with black dashed lines. Furthermore, the power spectrum shows a prominent peak at $2\langle\Delta \Omega\rangle_{12}$ for both oscillators of the second cluster and a flat curve for the representative of the first cluster. These numerical results are in complete agreement with the analytic findings.

Analogously, to the antipodal two-clusters, for any fixed set of parameters and each $n_{1}$, equation (5.17) can lead to two multiclusters of mixed type with two different frequency differences. Hence, a large number of those clusters can coexist for the same parameter values. Figure 5.11 illustrates such a coexistence, where we present the solutions to the equation (5.17). Again, blue solid lines represent those solutions for which the asymptotic expansion led to an existing and stable two-cluster solutions of mixed type. Additionally, Figure 5.11 shows the one-cluster solutions of splay and antipodal type (in both cases $\Delta \Omega_{12}=0$ ) together with their common regions of stability. As in the case of two-clusters of antipodal type, the asymptotic expansion presented in Proposition 5.4.1 turns into a formal expansion whenever $|\Delta \Omega|>\epsilon$. The interval $[-\epsilon, \epsilon]$ is therefore highlighted in Fig. 5.11. 
(a)

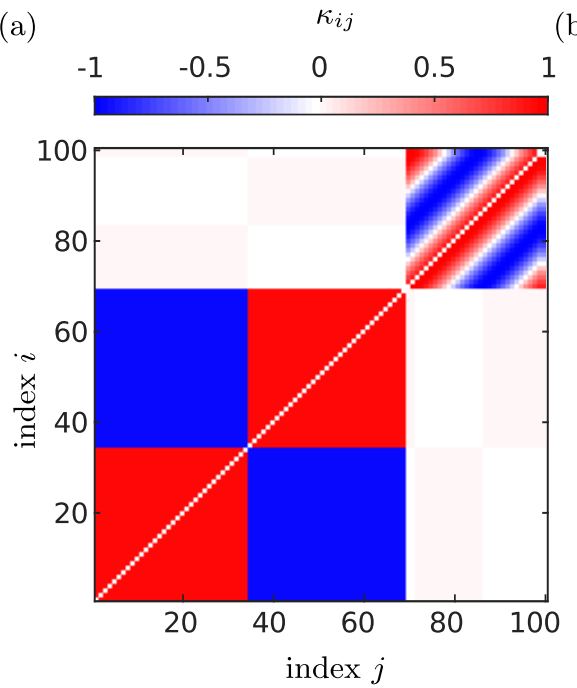

(b)

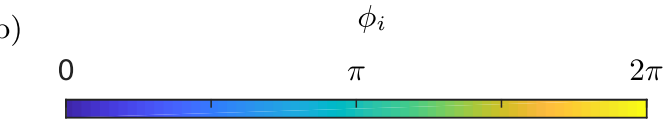

(c)
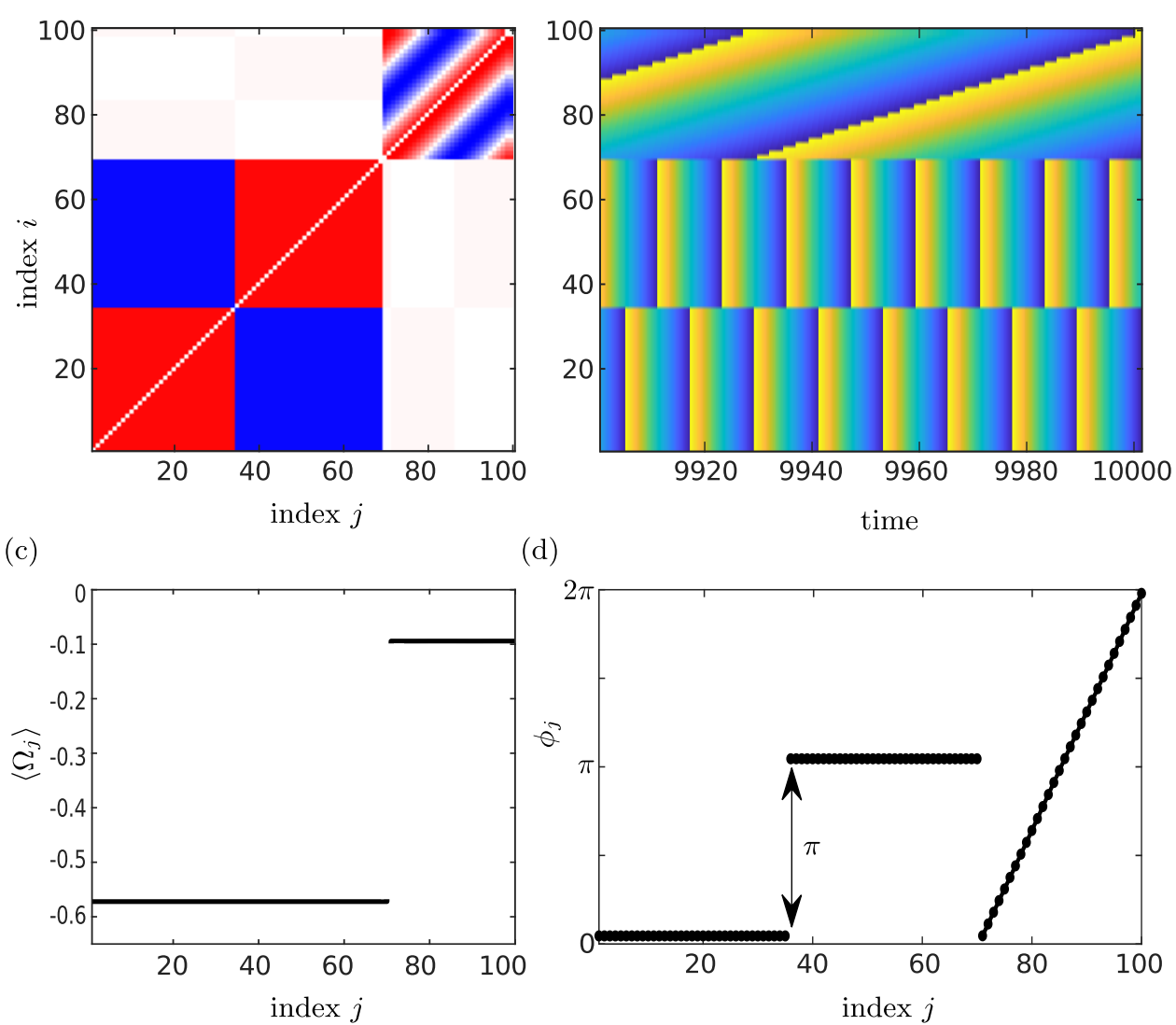

(d)

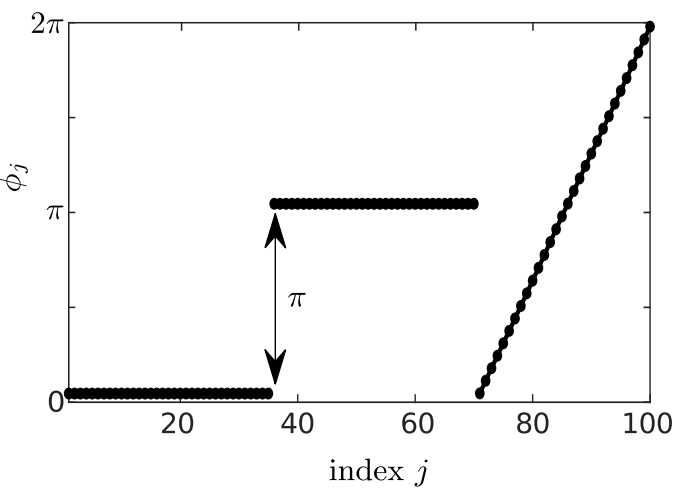

Figure 5.9: 2-Cluster solution of mixed type. (a) Coupling weights at $t=10000$ showing two clusters, (b) Distribution of the phases within each cluster, space-time representation. (c) Average frequency of each oscillator, (d) Oscillator phases $\phi_{i}$ for fixed time $t=10000$. Parameter values: $\epsilon=0.01, \alpha=0.3 \pi, \beta=-0.4 \pi, N=100$. Figure taken from [BER19].

(a)

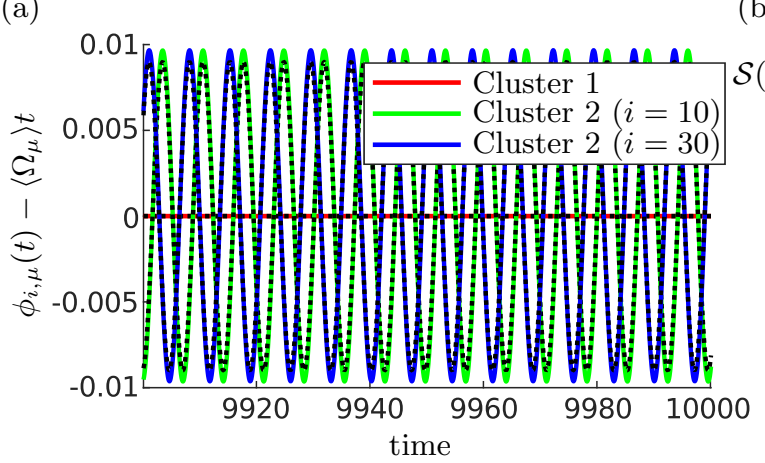

(b)

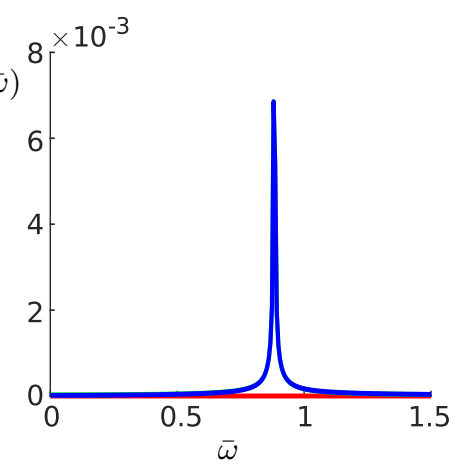

Figure 5.10: For mixed type 2-cluster solution from Fig. 5.9, panel (a) shows time series of an oscillator from one of the clusters after subtracting the average linear growth $\phi_{\mu, i}(t)-\left\langle\Omega_{\mu}\right\rangle t$. The black dashed lines show the corresponding analytic results from the asymptotic expansion in Proposition 5.5.1. (b) Power spectrum of the time series given in (a). Figure moified from [BER19]. 

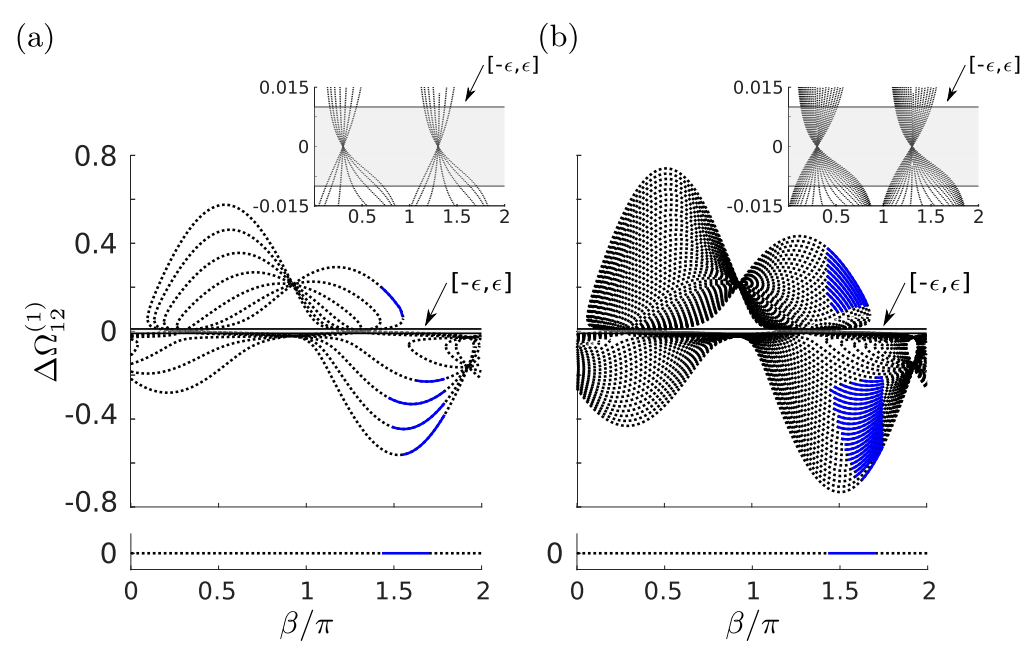

Figure 5.11: Two-cluster solutions of mixed type (upper panels) and one-cluster solutions (lower panels) of either splay or antipodal type given by the asymptotic expansion in Corollary 5.5.2 and Proposition 4.1.1, respectively. For this, the difference of the frequencies $\Delta \Omega_{12}^{(1)}$ is displayed corresponding to (5.17) and (4.6). The dotted lines (black) indicate unstable solutions while the solid lines (blue) indicate stable solutions. Here, every second solution is plotted for the sake of visibility. The insets show a blow-up of the interval $[-\epsilon, \epsilon]$. Parameter values: (a) $N=20, \epsilon=0.01$; (b) $N=50, \epsilon=0.01 ; \alpha=0.3 \pi$ is fixed for all panels. Figure taken from [BER19].

\subsection{Stability of multicluster states}

In Section 5.2 we discussed multicluster solutions of splay type and showed under which condition they exist. The solutions for two-cluster solutions of splay type and their stability are presented in Fig. 5.4. In Fig. 5.4(b) the solution for the case of 50 oscillators is shown. The solid lines (blue) correspond to solutions that are stable. It can be seen that whenever a 2-cluster solution is stable the one-cluster solution (with $\Delta \Omega_{12}=0$ ) is also stable.

A more detailed validation of this statement is presented in Fig. 5.12, where we show the stability regions of both one- and two-cluster solutions in the $(\alpha, \beta)$ plane. The stability for each type of cluster solution is determined numerically. The numerical approach was already introduced in Section 4.2. For the two-cluster solutions the norm for the phase configuration is calculated in the relative coordinates given by $\Theta_{i, \mu}=\phi_{i, \mu}-\phi_{1, \mu}$ with $\mu=1,2$. Additionally, we calculated the maximal value of all inter-cluster connections and compared it to the theoretical maximum given by $\rho_{12}$. If after numerical integration the maximal inter-cluster coupling is bigger than $\rho_{12}+0.01$, the two-cluster is considered as unstable. Here, region where the both types of solutions are stable are colored in dark blue. Regions of only stable one-cluster solutions are colored in light blue. Since two-cluster solutions do not exist for certain values of $\alpha$ and $\beta$, we can find a light blue stripe in the middle of Fig. 5.12. Further, we have not found any configuration of $\alpha$ and $\beta$ for which two-cluster solutions are stable and one-cluster are not. This supports the claim that the stability of a one-cluster solution is necessary condition for the stability of a two-cluster solution. This can be explained by the fact that for the stability of the multiclusters, it is necessary that its one-cluster components are each stable with respect to the perturbations that disturb the structure of just one cluster (see similarly in [LUE12a]). A more rigorous formulation of this issue is beyond the scope of this thesis.

Figure 5.4(b) further provides us with information about the stability of two-cluster solutions depending on the ratio between cluster sizes. First, due to (5.7) there exist two branches of 


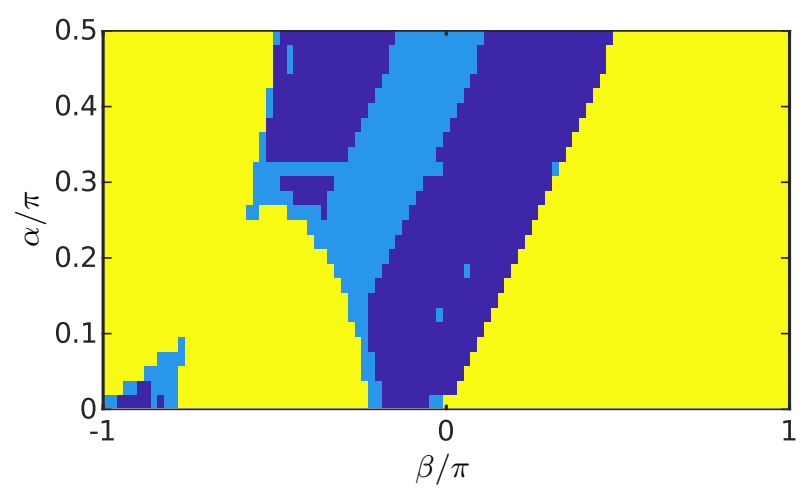

Figure 5.12: Stability diagram for the one-cluster and two-cluster solution of the splay type depending on the parameters $\alpha$ and $\beta$. Yellow region corresponds to the instability of both solutions, dark blue to the stability of both solutions, and light-blue to the stability of only the one-cluster solution. Parameter $\epsilon=0.01$ is fixed in all simulations. Figure taken from [BER19].

two-cluster solution of splay type. Only solutions with higher frequency difference are stable which can be seen in the inset of Fig. 5.4(b). For an increasing number of oscillators in the second cluster of relative size $n_{2}=1-n_{1}$ the stability changes. Above a certain value of $n_{2}$ both branches are unstable. This observation explains why only multicluster solutions with unequal as well as hierarchical cluster sizes were found in simulations, see Fig. (5.3) and [KAS17].

\subsubsection{On the stability of multicluster states with evenly sized clusters}

In the following, we give a necessary condition for the stability of all one-cluster states of splay type complementing the result on rotating-wave states given in Proposition 4.2.2. This extension enables us to determine the stability of evenly sized multiclusters of splay type.

In general all splay one-cluster states have the property $R_{2}(a)=0$ for the phase given by the vector $\boldsymbol{a}$. Therefore, the splay states form $N-2$ dimensional family of solution. Hence, around each splay states there are $N-2$ neutral variational directions $(\delta \phi, \delta \kappa)^{T}$ which are determined by the condition $\sum_{j=1}^{N} e^{\mathrm{i} 2 a_{j}} \delta \phi_{j}=0$. Note, $\delta \kappa_{i j}=-\cos \left(a_{i}-a_{j}+\beta\right)\left(\delta \phi_{i}-\phi_{j}\right)$ in neutral direction.

Proposition 5.6.1 Consider an asymptotically stable one-cluster state of splay type. Then, $\epsilon+\sin (\alpha-$ $\beta) / 2>0$.

Proof. For some notation we refer the reader to appendix A.2. Due to the block form of the linearised equation (A.6) and the Schur decomposition (4.11), any eigenvalue comes with a second. We have already seen this in Lemma A.2.1 and Proposition A.2.3. Variation along the neutral direction gives $N-2$ times the eigenvalue 0 . Suppose we have $\delta \phi$ such that $\sum_{j=1}^{N} e^{\mathrm{i} 2 a_{j}} \delta \phi_{j}=0$ and $\delta \kappa_{i j}=-\cos \left(a_{i}-a_{j}+\beta\right)\left(\delta \phi_{i}-\phi_{j}\right)$. Applying Schur decomposition (4.11), we get

$$
(M-\lambda \mathbb{I})_{N^{2}+N}\left(\begin{array}{l}
\delta \boldsymbol{\phi} \\
\delta \kappa
\end{array}\right)=\left(\begin{array}{cc}
\mathbb{I}_{N} & -(\epsilon+\lambda) B \\
0 & \mathbb{I}_{N^{2}}
\end{array}\right)\left(\begin{array}{cc}
\left(A-\lambda \mathbb{I}_{N}\right)+\frac{1}{\epsilon+\lambda} B C & 0 \\
0 & -(\epsilon+\lambda)
\end{array}\right)\left(\begin{array}{c}
\delta \boldsymbol{\phi} \\
-\frac{1}{\epsilon+\lambda} C \delta \boldsymbol{\phi}+\delta \kappa
\end{array}\right)=0 .
$$


With this, we have to find $\lambda$ such that the last equality in (5.18) is fulfilled. This is equivalent to solving $\left(\left(A-\lambda \mathbb{I}_{N}\right)(\epsilon+\lambda)+B C\right) \delta \phi=0$ of which in general only $N-2$ equations are linearly independent. The equivalence can be seen by multiplying $\epsilon+\lambda$ from both sides and keeping in mind that $\delta \kappa$ is already determined by $\delta \phi$. Using the definition of $\delta \phi$ the matrices $A$ and $B C$ can be effectively reduced in such a way that they are independent of the actual values for the phases $a_{j}$. In fact,

$$
\begin{aligned}
a_{i j} & = \begin{cases}-\frac{N-1}{2 N} \sin (\alpha-\beta) & i=j \\
\frac{1}{2 N} \sin (\alpha-\beta), & i \neq j\end{cases} \\
(b c)_{i j} & = \begin{cases}\epsilon \frac{N-1}{2 N} \sin (\alpha-\beta), & i=j \\
-\frac{\epsilon}{2 N} \sin (\alpha-\beta), & i \neq j\end{cases}
\end{aligned}
$$

In turn, this gives $\left(\left(A-\lambda \mathbb{I}_{N}\right)(\epsilon+\lambda)+B C\right)$ a circulant structure which can be used to diagonalise the matrix, in analogy to Proposition A.2.3. For circulant matrices we immediately know the eigenvalues. They are

$$
\mu_{l}=-\lambda^{2}-\left(\frac{N-1}{2 N} \sin (\alpha-\beta)-\frac{1}{2 N} \sin (\alpha-\beta)\left(\sum_{k=0}^{N-1} e^{\mathrm{i} 2 \pi k l / N}-1\right)+\epsilon\right) \lambda
$$

with $l=0, \ldots, N-1$ and $\operatorname{det}\left(\left(A-\lambda \mathbb{I}_{N}\right)(\epsilon+\lambda)+B C\right)=\mu_{0}(\lambda) \cdots \mu_{N-1}(\lambda)$. Remember we have in general $N-2$ independent equations. Thus, solving $\mu_{l}(\lambda)=0$ for $\lambda$ results in $N-2$ eigenvalues $\lambda=0,1$ eigenvalue $\lambda=-\epsilon$ and $N-3$ eigenvalues $\lambda=-\epsilon-\sin (\alpha-\beta) / 2$. Note that for 4 -phasecluster states, as considered in Corollary A.2.5, the number of independent equations is $N-1$. This is due to the fact that in this case the equations for the imaginary and real part from $\sum_{j=1}^{N} e^{\mathrm{i} 2 a_{j}} \delta \phi_{j}=0$ agree.

Finally, we note why the equally-sized splay-clusters are not found to be stable. Indeed, from Eq. (5.7) we know that $2 \epsilon+\sin (\alpha-\beta)<0$ is a necessary condition to have such equally-sized $\left(n_{1}=1 / 2\right)$ clusters. However, any one-cluster splay state is unstable for $2 \epsilon+\sin (\alpha-\beta)<0$ by Proposition 5.6.1.

\subsubsection{An effective approach for the stability of multicluster states}

As mentioned above, the stability of one-cluster states is important for the stability of multicluster states. For two weakly coupled clusters, the stability of one-clusters serves as a necessary condition for the stability of the two-cluster state. In Figure 5.4 the possible two-clusters of splay type are plotted. We notice that for $\epsilon=0.001$ a stable two-cluster state exists for almost every relative cluster size $n_{1}$, while this is not true for $\epsilon=0.01$ and even more so for $\epsilon=0.1$.

Another observation from Figs. 5.4(b,c) is that the possible $\beta$-values where the two-cluster states can be stable mainly correspond to the $\beta$ values where the one-cluster state is stable. This is true for small values of $\epsilon$, however, a careful inspection of Fig. 5.4(d) for the case of larger $\epsilon$, here $\epsilon=0.1$, shows that some two-cluster states appear to be stable for a parameter region where the corresponding one-cluster state is unstable. This can be explained as follows. According to (5.1), in the case of one-cluster states, the inter-cluster interactions are summed over all $N$ 
oscillators of the whole system. Additionally, the interactions are scaled with the factor $1 / N$. Therefore, the total interaction scales with 1 . For two-cluster states, the inter-cluster interactions for each individual cluster are only a sum over the $N_{\mu}(\mu=1,2)$ oscillators whereas the scaling remains $1 / N$. Hence, the total inter-cluster interaction scales with $n_{\mu}=N_{\mu} / N$, the relative size of the cluster. Therefore, the effective oscillatory system, when neglecting the interaction to the other cluster, reads

$$
\begin{aligned}
\frac{\mathrm{d} \phi_{i, \mu}}{\mathrm{d} t} & =-\frac{n_{\mu}}{N_{\mu}} \sum_{j=1}^{N_{\mu}} \kappa_{i j, \mu \mu} \sin \left(\phi_{i, \mu}-\phi_{j, \mu}+\alpha\right), \\
\frac{\mathrm{d} \kappa_{i j, \mu \mu}}{\mathrm{d} t} & =-\epsilon\left(\kappa_{i j, \mu \mu}+\sin \left(\phi_{i, \mu}-\phi_{j, \mu}+\beta\right)\right) .
\end{aligned}
$$

This system is equivalent to (5.1)-(5.2) with $N_{\mu}$ oscillators by rescaling $\epsilon \mapsto \epsilon / n_{\mu}$. Thus, the stability of the inter-cluster system has to be evaluated with respect to the rescaled effective parameter $\epsilon_{\mathrm{eff}}:=\epsilon / n_{\mu}$. Since $n_{\mu}<1$ for $\mu=1,2$, we have $\epsilon_{\mathrm{eff}}>\epsilon$. As we have discussed, the stability for the one-cluster changes with increasing $\epsilon$. With this, the influence of the cluster size as well as the slight boundary shift in the regions of stability, see Fig. 5.4, can be explained.

\subsection{Summary}

In this chapter, we have focused on multicluster solutions in a network of adaptively coupled phase oscillators. The multicluster states are composed of several one-clusters with distinct frequencies. Starting from random initial conditions, our numerical simulations show two different types of states. These are the splay and the antipodal type multicluster states. A third mixed type multicluster state is found by specially prepared initial conditions. For all these states the collective motion of oscillators, the shape of the network, and the interaction between the frequency clusters is presented in detail. It turns out that the oscillators are able to form groups of strongly connected units. The interaction between the groups is weak compared to the interaction within the groups. The numerical analysis of multicluster states reveals the building blocks for these states.

While the one-cluster solutions are relative equilibria of our system due to the phase-shift symmetry, the multicluster solutions contain components with different frequencies, and, hence, they cannot be reduced to an equilibrium by transforming into another co-rotating frame. As a result, the study of multiclusters is more involved. However, to our surprise, we have still been able to find an explicit form of multiclusters with the components of the splay type. Remarkably, in addition to its ring-like spatial structure that dynamically emerges, the network behaves in such a case (quasi-)periodically in time such that the whole solution can be interpreted as a spatial-temporal wave.

The analysis of multicluster solutions of antipodal type is more subtle due to the modulation of the frequency. More specifically, we look at multiclusters with bounded frequency modulation. For these types of multiclusters, we derive an asymptotic expansion in the parameter $\epsilon$ that gives explicit existence conditions. In addition, we have shown the existence of mixed multiclusters, which consist of clusters of splay type and clusters of antipodal type. For the mixed multiclusters, the temporal behavior within one cluster has been shown to be slightly non-identical, namely, 
the oscillators possess the same averaged frequency, but they still can have a bounded quasiperiodically modulated phase difference.

For the splay clusters we analytically show the existence of two-cluster states. Remarkably, while the existence of the one-cluster states does not depend on the time-scale separation parameter $\epsilon$, the multicluster states crucially depend on the time-scale separation. In fact, we provide an analysis showing that there exists a critical value for the time-scale separation. Moreover, we show that in the case of two-cluster states of splay type the adaptation of the coupling weights must be at most half as fast as the dynamics of the oscillators. This fact is of crucial importance for comparing dynamical scenarios induced by short-term or long-term plasticity [FRO16].

The stability of two-cluster states is analyzed numerically and presented for different values of the time-scale separation parameter. By assuming weakly interacting clusters, we describe the stability of the two-cluster with the help of the analysis of one-cluster states. The main messages from this analysis are as follows: there is a high degree of coexistence of stable multiclusters that can be reached from different initial conditions; in particular, a certain amount of imbalance in the number of oscillators within the clusters is needed to achieve stability. This explains the appearance of only hierarchical structures in numerical simulations. In fact, the simulations indicate that there are no stable two-cluster states with clusters of the same size. We provide a rigorous argument to understand this property of the system.

Moreover, the findings on multicluster solutions as they are reported in this chapter are in very good agreement with previous results on adaptive neural networks [POP15, CHA17a]. Here, stable multicluster solutions of coherently spiking neurons with weak but time-dependent inter-cluster coupling are reported. With this work we shed some light on these generic timedependent network patterns. 


\section{Part II}

\section{INTERPLAY OF ADAPTIVITY AND CONNECTIVITY}





\section{Adaptation on nonlocally coupled ring 6 \\ networks}

In this chapter, we go beyond the model of globally coupled oscillators which were in the focus of the Chapters 3-5. In particular, here, we study the interplay of adaptivity with a non-locally coupled ring structure. Ring-like structures are important motifs in neural networks [COM03, SPO11, POP11, YAN11]. Specifically, nonlocally coupled rings where each node is coupled to all nodes within a certain coupling range, are known to be important for systems appearing in many applied problems and theoretical studies [PAS95, BRE97, YAN08a, BON09, ZOU09b, HOR09b, PER10c, OME11, KAN13, OME13, YAN15a, KLI17, BUR18, OME18a].

In the subsequent sections, we consider the following system of $N$ adaptively coupled identical phase oscillators [AOK09, AOK11, NEK16, KAS17]

$$
\begin{aligned}
\frac{\mathrm{d} \phi_{i}}{\mathrm{~d} t} & =1-\frac{1}{\sum_{j=1}^{N} a_{i j}} \sum_{j=1}^{N} a_{i j} \kappa_{i j} \sin \left(\phi_{i}-\phi_{j}+\alpha\right) \\
\frac{\mathrm{d} \kappa_{i j}}{\mathrm{~d} t} & =-\epsilon\left(\kappa_{i j}+\sin \left(\phi_{i}-\phi_{j}+\beta\right)\right)
\end{aligned}
$$

where $i, j=1, \ldots, N, \phi_{i} \in S^{1}$ are the phases of the oscillators, $a_{i j} \in\{0,1\}$ are the entries of the adjacency matrix $A$ determining the base topology, $\kappa_{i j} \in[-1,1]$ are slowly changing adaptive coupling strengths, $0<\epsilon \ll 1$ is the rate of the adaptation, and $\alpha, \beta$ are coupling and adaptation phase lags. Note that the natural oscillation frequency has been normalized to 1 by the rotating coordinate frame.

The base topology given by the adjacency matrix A determines the structure of the network, on which the adaptation takes place. Equation (6.2) for the adaptation is used only for "active" weights $\kappa_{i j}$ corresponding to $a_{i j}=1$. Similarly, the sum in (6.1) goes over these links. Here we consider the topology of a nonlocally coupled ring given by

$$
a_{i j}= \begin{cases}1 & \text { for } 0<(i-j) \bmod N \leq P, \\ 0 & \text { otherwise. }\end{cases}
$$

This means that any two oscillators are coupled if their indices $i$ and $j$ are separated at most by the coupling radius $P$. The coupling Eq. (6.3) defines a nonlocal ring structure with coupling range $P$ to each side and two special limiting cases: local ring for $P=1$ and globally coupled network for $P=N / 2$ (if $N$ is even, else $P=(N+1) / 2$ ). The matrix of the form (6.3) is circulant [GRA06] and has constant row sum, i.e., $\sum_{j=1}^{N} a_{i j}=2 P$ for all $i=1, \ldots, N$. Due to the same symmetries as they are present in (2.25)-(2.26), the analysis can be restricted to the parameter regions $\alpha \in[0, \pi / 2)$ and $\beta \in[-\pi, \pi)$.

Two measures of coherence are used in this chapter: first, the $n$th moment of the $i$ th $(i=1, \ldots, N)$ 
complex local order parameter as given by

$$
Z_{i}^{(n)}(\boldsymbol{\phi}):=\frac{1}{2 P} \sum_{j=1}^{N} a_{i j} e^{\mathrm{i} n \phi_{j}}=R_{i}^{(n)}(\boldsymbol{\phi}) e^{\mathrm{i} \vartheta_{i}^{(n)}(\boldsymbol{\phi})}
$$

where $\phi=\left(\phi_{1}, \ldots, \phi_{N}\right)^{T}, R_{i}^{(n)}(\phi)$ is the $n$th local order parameter and $\vartheta_{i}^{(n)}(\phi)$ the $n$th local mean-phase; second, the complex (global) order parameter

$$
Z^{(n)}(\boldsymbol{\phi}):=\frac{1}{N} \sum_{j=1}^{N} e^{\mathrm{i} n \phi_{j}}=R^{(n)}(\boldsymbol{\phi}) e^{\mathrm{i} \vartheta^{(n)}(\boldsymbol{\phi})}
$$

where $R^{(n)}(\phi)$ is the $n$th (global) order parameter and $\vartheta^{(n)}(\phi)$ the $n$th (global) mean-phase, see also Section 2.2. Both measures are used throughout the chapter to characterize asymptotic states of (6.1)-(6.2).

This chapter includes contents that have been published in [?]. The chapter is organized as follows. Numerical results and a rigorous definition for multicluster and solitary states on complex networks are presented in Section 6.1. In Section 6.2 we provide a more detailed analysis of one-cluster states. Here, relations between local and global properties are derived. The salient role of rotating-wave clusters are underlined and the crucial dependence of their stability on the coupling range and the wavenumber are rigorously described. Some of the proofs are presented in the App. A.5 and A.6. After this, we focus on the analysis of solitary states in Sec. 6.3. A reduced model for two-clusters is derived and a variety of bifurcations in which solitary states are born and stabilized are presented. Section 6.5 summarizes our findings.

\subsection{Multicluster and solitary states}

This section is devoted to the numerical analysis of system (6.1)-(6.2) and the description of several dynamical states which occur for this system. More specifically, we report one-cluster, multicluster, and solitary states. While this section describes the states in a phenomenological fashion, more rigorous results are presented in the subsequent Sections 6.2-6.3.

Note that the observed one-cluster and multicluster states are similar to those reported in Chapters 4 and 5 for the all-to-all coupling base topology. However, there are important differences due to the ring structure of our system, which will be discussed in detail.

For the numerical simulations in this section a system of $N=100$ oscillators with coupling range $P=20$ is studied. The value of $\epsilon$ is set to 0.01 and the system parameter $\alpha$ and $\beta$ are varied in the ranges $[0, \pi / 2]$ and $[-\pi, \pi]$, respectively. All results are obtained starting from uniformly distributed random initial conditions and a simulation time of $t=20000$. Several types of synchronization patterns are found in the numerical simulations, depending on the values of $\alpha$ and $\beta$. 

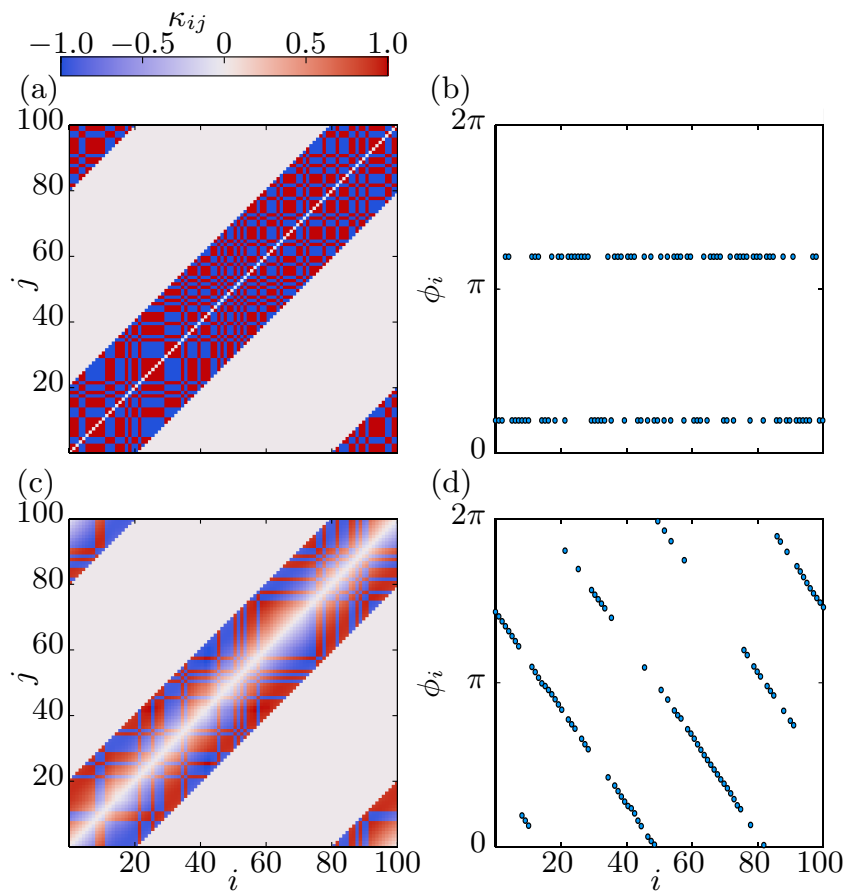

Figure 6.1: Illustration for two types of one-cluster states. The panels $(a, c)$ show the asymptotic coupling matrices and $(b, d)$ snapshots of the phases at a fixed time. Results for the one-cluster states of antipodal type are presented in $(\mathrm{a}, \mathrm{b})$ where $\alpha=0.19 \pi, \beta=-0.66 \pi$ and of splay type in (c,d) where $\alpha=0.35 \pi, \beta=0.01 \pi$. Parameters: $N=100, P=20$, $\epsilon=0.01$. Figure modified from [?].

\subsubsection{One-cluster states}

A one-cluster is defined as a frequency synchronized state

$$
\phi_{i}=\Omega t+\chi_{i}, \quad i=1, \ldots, N
$$

with a collective frequency $\Omega \in \mathbb{R}$ and individual phase shifts $\chi_{i} \in[0,2 \pi)$, see Section 4.1 . The two types of one-cluster states found in the numerical simulations are either of antipodal or splay type whose asymptotic configurations are displayed in Fig. 6.1(a,b) and Fig. 6.1(c,d), respectively. The antipodal and splay-type clusters have been introduced previously in Chapter 4 . In the antipodal cluster, all phases $\phi_{i}$ are either in-phase or in anti-phase, i.e., $\chi_{i} \in\{\chi, \chi+\pi\}$ with $\chi \in[0,2 \pi)$ and hence $R^{(2)}(\phi)=1$. In the splay cluster the phases are distributed across the interval $[0,2 \pi)$ such that the global second order parameter, as defined in equation (6.5), vanishes, i.e., $R^{(2)}(\phi)=0$. In Fig. 6.1 (a,c) the coupling structures corresponding to the two types of one-clusters are displayed. Note that the coupling weights are solely described by the phase differences of the oscillators and are given by

$$
\kappa_{i j}=-\sin \left(\chi_{i}-\chi_{j}+\beta\right)
$$

The one-cluster states, which exist in our ring case and the all-to-all base topology case from Chapter 4 have the same representation except for the fact that some of the coupling weights are absent in the case of the ring, see empty entries in Fig. 6.1(a,c). 


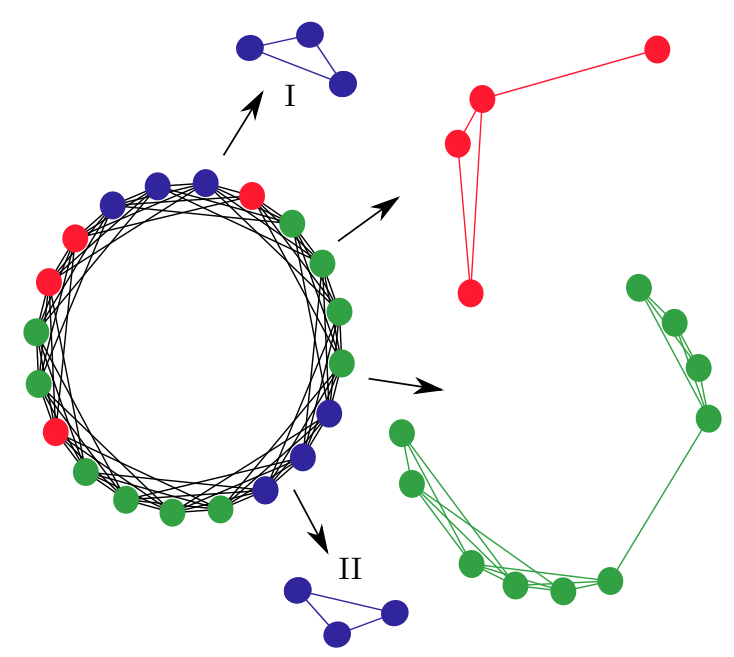

Figure 6.2: Schematic figure illustrating the definition of multicluster and subnetworks induced by groups of nodes with the same average frequency. The full network (left) consists of $N=20$ nodes and has a nonlocal ring structure with $P=4$. The colors of the nodes indicate their average frequencies. Clusters are shown by the equally colored nodes that form connected sub-networks. Even though the two blue groups I and II possess the same averaged frequencies, they form two different clusters, since they are not connected. Figure taken from [BER20c].

\subsubsection{Multicluster states}

As described in Chapter 5, one-clusters can serve as building blocks for multi-frequency clustered states where the phase dynamics and the coupling matrix $\kappa$ are divided into different groups; $\kappa_{i j, \mu \nu}$ refers to the coupling weight for the connection from the $i$ th oscillator of the $\mu$ th cluster to the $j$ th oscillator of the $\nu$ th cluster. Analogously, $\phi_{i, \mu}$ denotes the $i$ th phase oscillator in the $\mu$ th cluster. The temporal behavior for each oscillator in an $M$-cluster state takes the form, see also Definition 5.0.1

$$
\phi_{i, \mu}(t)=\Omega_{\mu} t+\chi_{i, \mu}+s_{i, \mu}(t) \quad \begin{aligned}
& \mu=1, \ldots, M \\
& \\
&
\end{aligned}
$$

where $M$ is the number of clusters, $N_{\mu}$ is the number of oscillators in the $\mu$ th cluster, $\chi_{i, \mu} \in[0,2 \pi)$ are phase lags, and $\Omega_{\mu} \in \mathbb{R}$ is the collective frequency of the oscillators in the $\mu$ th cluster. The functions $s_{i, \mu}(t)$ are assumed to be bounded.

Both types of one-clusters give rise to multi-frequency cluster states, see Fig. 6.1, similarly to the case of all-to-all base coupling. However, in case of more complex network structures the definition of a frequency-cluster has to be refined to account for the connectedness of the individual building blocks. Therefore, a multi-frequency-cluster (shortly: multicluster) consists of groups of frequency synchronized oscillators for which the subnetwork (or subgraph), induced by the individual groups of nodes, is connected. Here, we say a network is connected if there is directed path from each node to every other node of the network. In case of a directed graph, we require the property of weak connectedness for the induced subgraph for the cluster. For an introduction to the terminology we refer the reader to [KOR18].

Let us illustrate the above definition. Consider a nonlocal ring network of $N=20$ oscillators with coupling range $P=4$ as presented in Fig. 6.2. Suppose that for each node of the network we have a certain average frequency which is indicated by the color. In Fig. 6.2, we have three different average frequencies denoted by the green, blue, and red colors. The individual clusters 
are given by the connected subnetworks induced by equally colored nodes. Note that even though the blue nodes have the same average frequency, they are forming two different clusters (I,II) corresponding to two connected components. Note that the induced subnetworks are not necessarily regular even if the base topology is regular, see for instance the red or green subnetworks.

Figure 6.3 shows two-cluster states of antipodal (Figs. 6.3(a-c)) and splay type (Figs. 6.3(d-f)). In both cases, Figs. 6.3(c,f) show that there are two distinct groups of oscillators with different averaged frequencies (green, red). It is easily verified that these groups form connected subnetworks and, hence, they form a two-cluster state. Due to the frequency difference between the individual clusters, the groups of oscillators decouple effectively. This can be seen in Figs. 6.3(a,d) where only oscillators of the same cluster are strongly coupled compared to the coupling between the clusters given by the respective coupling weights $\kappa_{i j, \mu \mu}$ and $\kappa_{i j, \mu \nu} \approx 0(\mu \neq \nu)$.

Snapshots of the phase distributions are presented in Figs 6.3(b,e). Figure 6.3(b) is showing the phase distribution of an antipodal cluster. In contrast to the case of global coupling, see Chapters 4 and 5 , the phases do not possess the exact antipodal property anymore. In fact, the scattering of the antipodal phase distribution is caused by the structure of the induced subnetwork which is not regular anymore for the in-degree of each node, i.e., the subnetwork does not have constant row sum, see e.g. Fig. 6.2. Note that in case of a global base topology, all induced subnetworks are global. The phase snapshot for the splay multicluster is displayed in Fig. 6.3(e). Here, as in the case with global base structure, the phase distribution possess the property that $R^{(2)}(\phi)=0$.

Another, more complex, antipodal five-cluster is presented in Fig. 6.3(g-i). In Fig. 6.3(i), we observe three groups, a big one (green) and two smaller ones (red, blue), with different frequencies. Moreover, in accordance with the definition of a frequency cluster and the illustration in Fig. 6.2, the blue and the red groups possesses two connected components (I,II) each. This fact implies the presence of five individual frequency clusters in Fig. 6.3(g-i). Remarkably, the red clusters I and II as well as the blue clusters I and II are of the same size. This observation is in contrast to the hierarchical structures discovered and analyzed in [KAS17] and Chapter 5. Hence, in the case of the ring base structure, the evenly sized clusters can appear, which was not possible in the case of global base structure [KAS17] and identical oscillators, see also Chapter 5.

While several examples for one- and multicluster states have been described above, Fig. 6.4 shows that these states are observable in a wide range in the $(\alpha, \beta)$ parameter space. The diagram in Fig. 6.4 is produced by running simulations of (6.1)-(6.2) from random initial conditions. In case a one-cluster or multicluster is found, the region is colored or hatched, respectively, in accordance with the legend in Fig. 6.4. We further used a continuation method in the $(\alpha, \beta)$ parameter space to show the full extent where the various types of multiclusters can be observed. In Section 6.2.1 and 6.2.2, we provide a more rigorous description for the existence and stability properties of the one-clusters.

\subsubsection{Solitary states}

For systems with global base coupling, the clusters in the multicluster states were found to be hierarchical in nature, i.e. the clusters varied in size significantly [KAS17], see also Chapter 5. As 


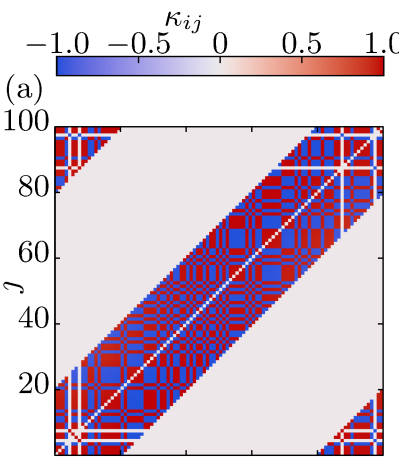

(b)
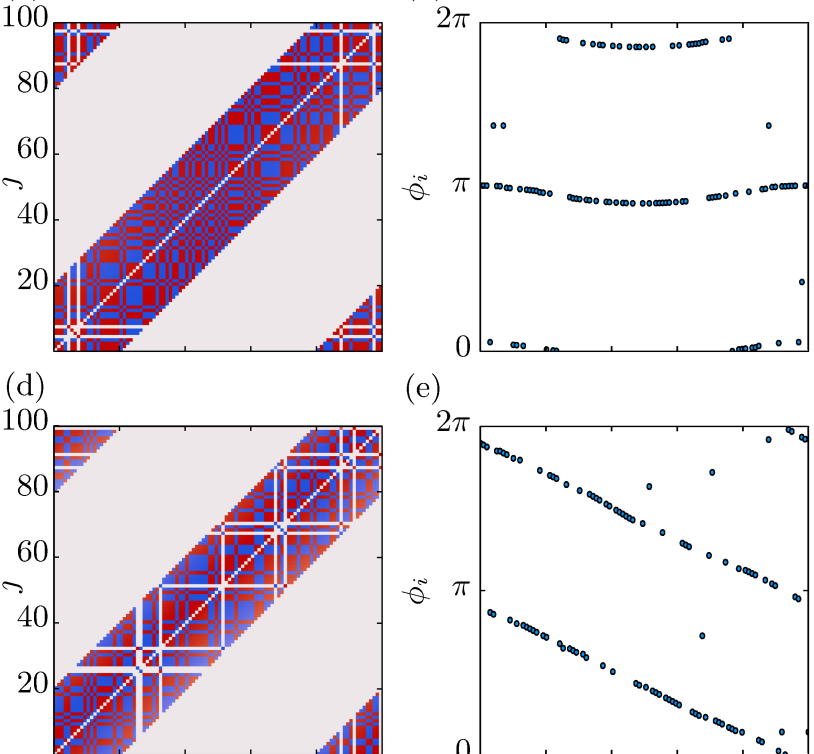

(g)

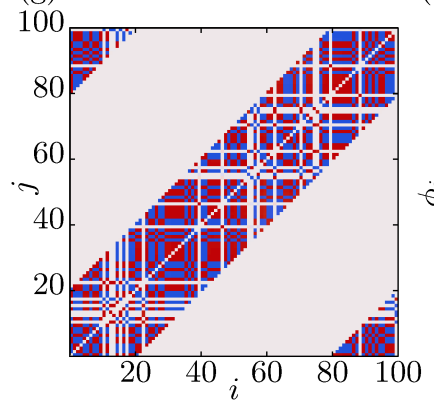

(e)

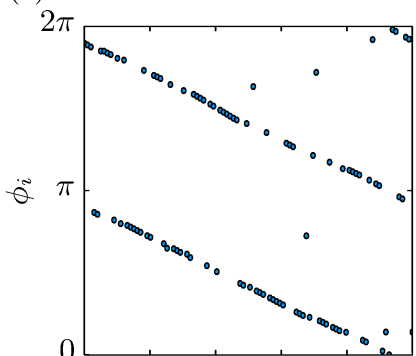

(h)

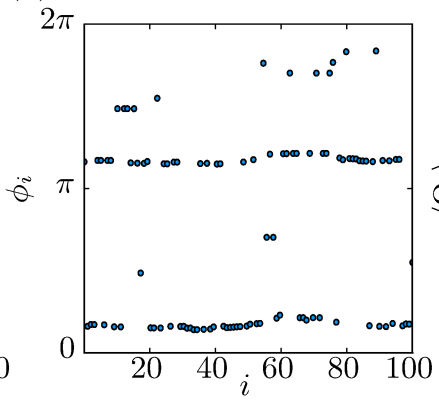

(c)

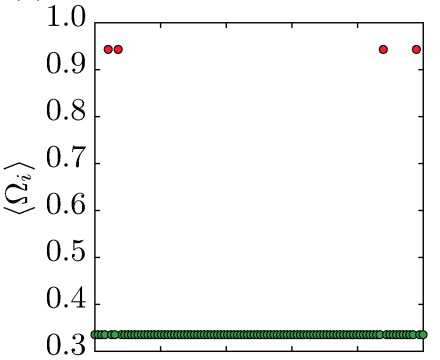

(f)

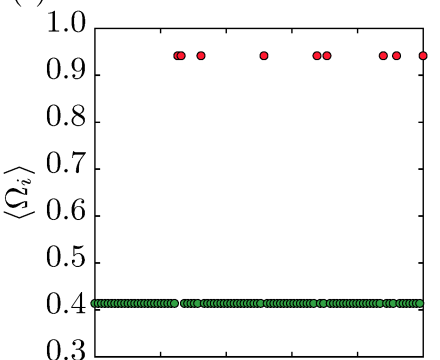

(i)

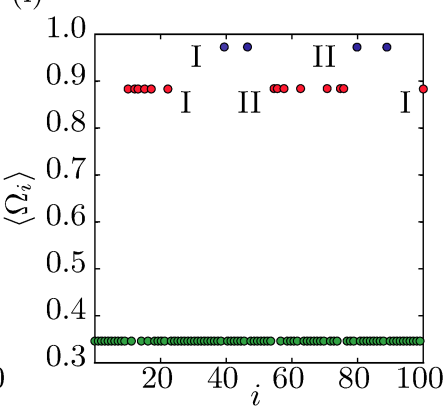

Figure 6.3: Illustration of the different types of multicluster states. The panels $(\mathrm{a}, \mathrm{d}, \mathrm{g})$ show the coupling matrix, $(\mathrm{b}, \mathrm{e}, \mathrm{h})$ phase snapshots and (c,f,i) average frequencies. (a-c): antipodal two-cluster for $\alpha=0.23 \pi, \beta=-0.56 \pi$; (d-f): splay two-cluster for $\alpha=0.19 \pi, \beta=-0.45 \pi$; (g-i): antipodal five-cluster (I, II denote the two connected components of the red and the blue clusters) for $\alpha=0.3 \pi, \beta=-0.53 \pi$. Parameters: $N=100, P=20, \epsilon=0.01$. Figure taken from [BER20c].

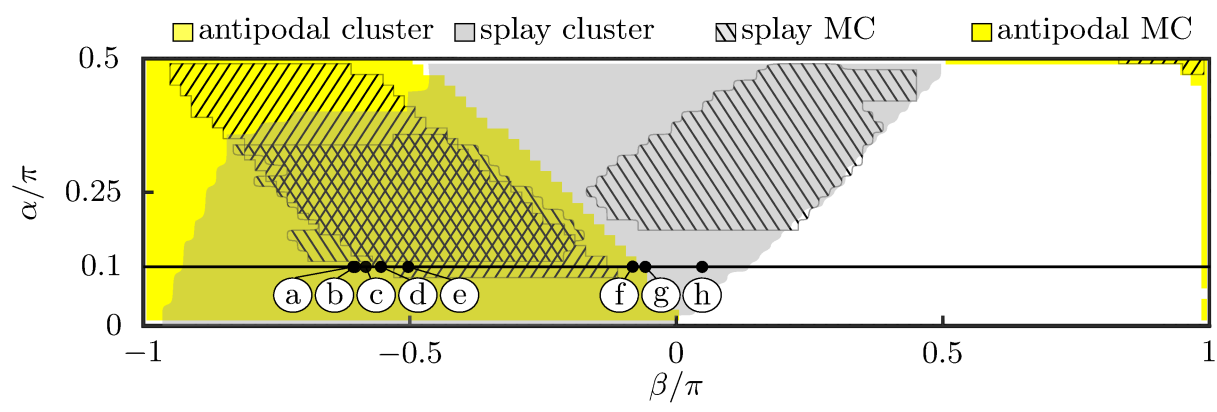

Figure 6.4: Map of regimes for one- and multicluster states of antipodal and splay type in $(\alpha, \beta)$ parameter space. Parameters: $N=100, P=20, \epsilon=0.01$. The horizontal black line at $\alpha=0.1$ shows the location for the parameter $\beta$ where the emergence of solitary states is analyzed, see Fig. 6.7 in Sec. 6.3. Figure taken from [BER20c]. 

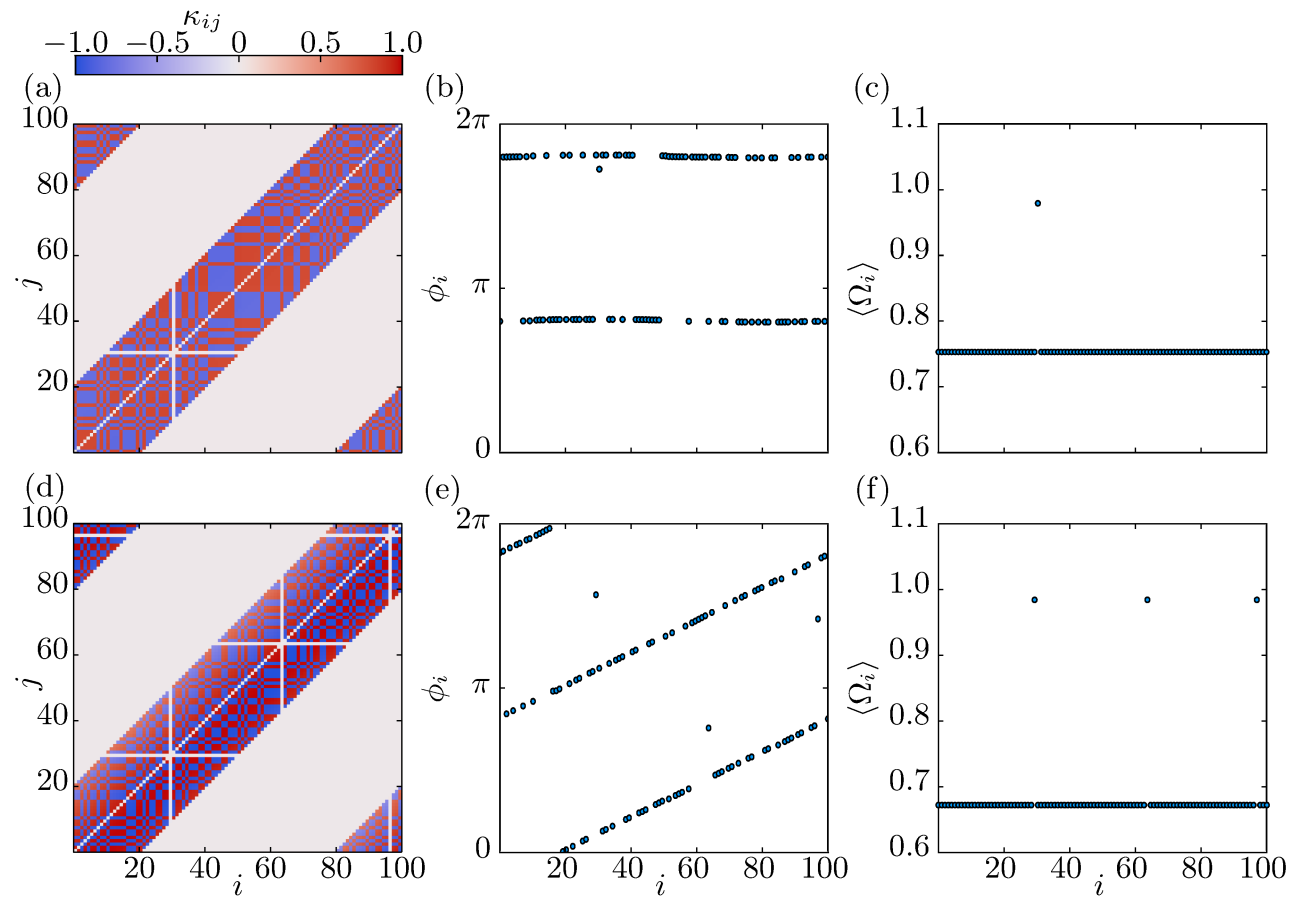

Figure 6.5: Illustration of solitary states. The panels $(\mathrm{a}, \mathrm{d})$ show coupling matrix, $(\mathrm{b}, \mathrm{e})$ phase snapshots, and $(\mathrm{c}, \mathrm{f})$ average frequencies. (a-c): single solitary state for $\alpha=0.1 \pi, \beta=-0.3 \pi$; (d-f): three uncoupled solitary states for $\alpha=0.15 \pi, \beta=-0.41 \pi$. Parameters: $N=100, P=20, \epsilon=0.01$. Figure modified from [BER20c].

we have mentioned above, in the nonlocal base coupling case, multicluster states with one large and many smaller, similar in size, clusters have been observed. Figure 6.5 shows a particular example of this phenomenon, called solitary states, where either one single oscillator (upper panels) or three single oscillators (lower panels) decouple from a large cluster. The solitary states are particular examples of multiclusters with a large group of frequency synchronized oscillators (background cluster) and individual solitary nodes with different frequency, i.e., clusters consisting of only one oscillator. These special kind of states, for which we provide an analysis of their emergence in Sec. 6.3, are of particular interest as they are found in various dynamical systems [MAI14a, ASH15, SEM15b, WOJ16, PRE16, MAI17, JAR18, TEI19, TAH19].

\subsection{One-cluster states: Local vs. global features}

\subsubsection{Classification of one-cluster states}

In this section we study the antipodal and splay one-cluster states in more details. Due to the $S^{1}$ symmetry of system (6.1)-(6.2), the following phase-locked solutions appear generically

$$
\phi_{i}(t)=\Omega t+\chi_{i}, \quad i=1, \ldots, N
$$

where $\chi_{i} \in[0,2 \pi)$ are fixed phase lags and $\Omega$ the cluster frequency. It is clear that such solutions describe a one-cluster state since the frequencies are the same. By substituting (6.6) into (6.1)- 
(6.2), we obtain

$$
\begin{gathered}
\kappa_{i j}=-\sin \left(\chi_{i}-\chi_{j}+\beta\right), \\
\Omega=\frac{1}{2} \cos (\alpha-\beta)-\frac{1}{4 P} \sum_{j=i-P}^{i+P} \cos \left(2 \chi_{i}-2 \chi_{j}+\alpha+\beta\right) .
\end{gathered}
$$

The equation (6.8) implies that the one-cluster state exists only if the following expression is independent of the index $i$

$$
\frac{1}{2 P} \sum_{j=i-P}^{i+P} \cos \left(2 \chi_{i}-2 \chi_{j}+\alpha+\beta\right)=\mathfrak{R}\left(R_{i}^{(2)} e^{\mathrm{i}\left(\vartheta_{i}^{(2)}-2 \chi_{i}-\alpha-\beta\right)}\right) .
$$

Equation (6.9) allows for the distinction of two types of distributions of the phase-lags for which it is independent of $i$. We call a cluster of

(i) Antipodal type, if $\chi_{i} \in\{0, \pi\}$ for all $i=1, \ldots, N$. In this case, the sum in (6.9) equals $\cos (\alpha+\beta)$, and of

(ii) Local splay type, if $\chi_{i} \in[0,2 \pi)$ are such that the second order parameter $Z_{i}^{(2)}=R_{i}^{(2)}(\chi) e^{i \vartheta_{i}^{(2)}}$ satisfies

$$
\begin{aligned}
R_{i}^{(2)}(\chi) & =R_{c}^{(2)}(\chi) \\
\vartheta_{i}^{(2)} & =2 \chi_{i}+\chi_{0}
\end{aligned}
$$

with $0 \leq R_{c}^{(2)}(\chi)<1$ and $\chi_{0} \in[0,2 \pi)$ independent on $i$.

Note that the additional degree of freedom for clusters of local splay type, i.e. $\chi_{0} \in[0,2 \pi)$ arbitrary, is due to the $S^{1}$ symmetry. The constant $\chi_{0}$ can be set to 0 without loss of generality. The both types of phase distribution lead to one-cluster states for the system (6.1)-(6.2) with frequency

$$
\Omega= \begin{cases}\sin \alpha \sin \beta & \text { antipodal type } \\ \frac{1}{2}\left(\cos (\alpha-\beta)-R_{c}^{(2)} \cos (\alpha+\beta)\right) & \text { local splay type. }\end{cases}
$$

In the context of globally coupled base topologies, antipodal and splay type phase distribution have been extensively discussed [ASH08] where the (global) splay clusters, also called fuzzy clusters [MAI14a], are defined by the global condition $Z^{(2)}(\chi)=0$, see also Chapter 4. Remarkably, if a phase distribution is of the local splay type (as described by (6.10)-(6.11)) then it is of splay type as well, see Appendix A.5 for more details. The converse is not true in general. Hence, the class of local splay clusters is "smaller" than the class of global splay clusters. In addition, local splay clusters do not necessarily form families of solutions. According to the definition of local splay cluster, generically $N$ complex algebraic equations have to be solved for $N$ unknown phase-lags $\chi_{i}$. Therefore, the set of equations for the phase-lags is overdetermined and the set of local splay states might be empty. However, it is not the case due to the symmetries of the system and the base coupling structure. The symmetry of the nonlocal ring structure allows for constructing explicit, symmetric examples for the clusters of local splay type. These are clusters of the rotating-wave type. 
(ii') The clusters are of rotating-wave type, if $\chi_{i}=i k \frac{2 \pi}{N}$, where $k=1, \ldots, N$ is the wavenumber. In the literature, the notion "splay state" is often restricted to this definition.

Let us show that the rotating wave clusters $\left(i i^{\prime}\right)$ are the local splay states (ii). For this we write the phase distribution as $\chi_{k}=(2 \pi k / N, \ldots 2 \pi k(N-1) / N, 0)^{T}$. Then, we have

$$
Z_{i}^{(n)}\left(\chi_{k}\right)=\frac{1}{2 P} \sum_{j=i-P}^{i+P} e^{\mathrm{i} n k j \frac{2 \pi}{N}}=e^{\mathrm{i} n k i \frac{2 \pi}{N}} R_{N}^{(n)}\left(\chi_{k}\right),
$$

where

$$
R_{N}^{(n)}\left(\chi_{k}\right)=\frac{1}{P}\left(\sum_{j=1}^{P} \cos \left(n k j \frac{2 \pi}{N}\right)\right) .
$$

we conclude that all rotating-wave states with $k \neq 0, N / 2$ are local splay states and thus solutions to (6.1)-(6.2). Rotating-wave clusters with $k=0, N / 2$ are of antipodal type. The $n$th moment local order parameter $\left|Z_{i}^{(n)}\left(\chi_{k}\right)\right|=R_{N}^{(n)}\left(\chi_{k}\right)$ is constant for all $i=1, \ldots, N$ and its value depends on the wavenumber $k$.

Note further that $Z_{i}^{(n)}\left(\chi_{k}\right)=Z_{i}^{(n k)}\left(\chi_{1}\right)$, which connects the moment of the order parameters with the wavenumber of the rotating-wave states. For globally coupled base structures, the rotating-wave states are found to be very important in describing the main features of antipodal and global splay type clusters such as stability. The next section is devoted to the description of the stability condition for rotating-wave states.

\subsubsection{Stability of one-cluster states}

In the following, the stability of one-clusters is analyzed. In order to study the local stability of one-cluster solutions described in Sec. 6.2.1, we linearize the system of differential equations (6.1)-(6.2) around the phase-locked states

$$
\begin{aligned}
\phi_{i}(t) & =\Omega t+a_{i}, \\
\kappa_{i j} & =-\sin \left(a_{i}-a_{j}+\beta\right) .
\end{aligned}
$$

These solutions are equilibria relative to the $S^{1}$ symmetry [GOL88a], therefore the linearization around such solutions leads to a linear system with constant coefficients, despite the time dependency of $\phi(t)$. Practically, one can first move to the co-rotating coordinate system by introducing the new variable $\phi(t)-\Omega t$ and then linearize around the equilibrium in the new coordinates. As a result, we obtain the following linearized system for the perturbations $\delta \phi_{i}$ and $\delta \kappa_{i j}$ :

$$
\begin{aligned}
\frac{d}{d t} \delta \phi_{i}= & \frac{1}{4 P} \sum_{j=i-P}^{i+P}\left[\sin (\beta-\alpha)+\sin \left(2\left(a_{i}-a_{j}\right)+\alpha+\beta\right)\right]\left(\delta \phi_{i}-\delta \phi_{j}\right) \\
& -\frac{1}{2 P} \sum_{j=i-P}^{i+P} \sin \left(a_{i}-a_{j}+\alpha\right) \delta \kappa_{i j}
\end{aligned}
$$



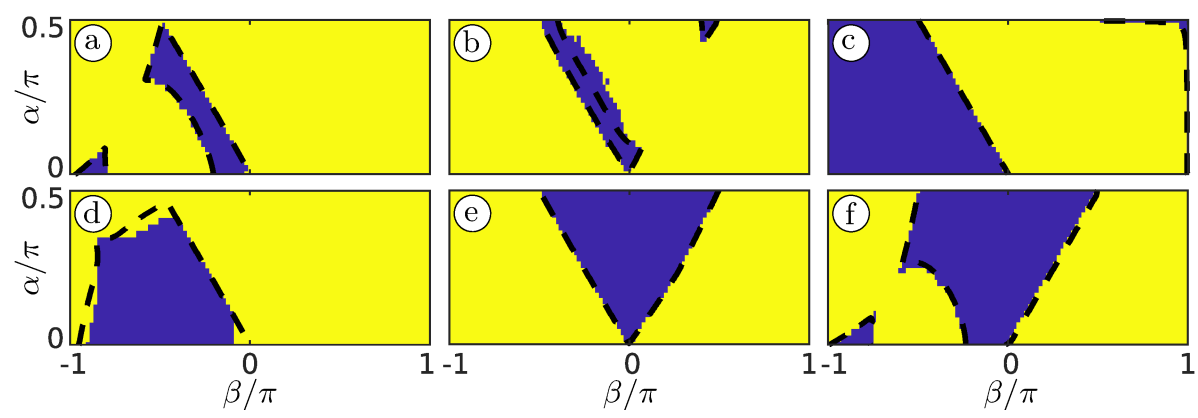

Figure 6.6: Stability of one-cluster states for different wavenumbers $k$ and coupling ranges $P$. Regions of stability for the one-cluster states are colored in blue, while instability in yellow. The borders of stability (black dashed lines) are obtained from the eigenvalues (6.17). Parameters are as follows: (a) $P=10, k=1$; (b) $P=10, k=4$; (c) $P=10, k=25$; (d) $P=5, k=1$; (e) $P=20, k=1$; and (f) $P=25, k=1$. The other parameters are $N=50$ and $\epsilon=0.01$. Figure modified from [BER20c].

and

$$
\frac{d}{d t} \delta \kappa_{i j}=-\epsilon a_{i j}\left(\delta \kappa_{i j}+\cos \left(a_{i}-a_{j}+\beta\right)\left(\delta \phi_{i}-\delta \phi_{j}\right)\right)
$$

System (6.15)-(6.16) is a $\left(N+N^{2}\right)$-dimensional linear system of ordinary differential equations, which can be written in the form $\boldsymbol{x}^{\prime}=\boldsymbol{L} \boldsymbol{x}, \boldsymbol{x} \in \mathbb{R}^{N+N^{2}}$, and the stability of which is determined by the eigenvalues of the matrix $\boldsymbol{L}$. For all antipodal and rotating-wave states the stability analysis can be done explicitly. However, the calculations are quite lengthy (see Appendix A.6). Summarizing the results of these calculations, the spectrum $S$ of the eigenvalues, corresponding to the rotating-wave one-clusters, is given by

$$
S=\left\{0,-\epsilon,\left\{\lambda_{l, 1}\right\}_{l=1}^{N},\left\{\lambda_{l, 2}\right\}_{l=1}^{N}\right\} .
$$

Here $\lambda_{l, 1}$ and $\lambda_{l, 2}$ are the solutions of the quadratic equation

$$
\lambda_{l}^{2}-\frac{\lambda_{l}}{2}\left[L(\alpha, \beta, l, k)+\left(R_{N}^{(l)}\left(\chi_{1}\right)-1\right) \sin (\alpha-\beta)-2 \epsilon\right]-\epsilon L(\alpha, \beta, l, k)=0,
$$

where the complex function $L$ as defined in Eq. (A.27) in Appendix A.6, $k$ is the wavenumber.

In Figure 6.6 we show the stability of rotating-wave one-clusters in an ensemble of $N=50$ oscillators. The figures demonstrate regions of stability in the $(\alpha, \beta)$ parameter plane for different wavenumber $k$ and coupling range $P$. The stability is obtained by numerical simulations as well as by the Lyapunov spectrum Eq. (6.17). The borders of stability, as they are provided by the analytical results, are displayed with a dashed black line. Numerically the stability was computed as follows: (i) the theoretical shape of the one-cluster given by (6.6)-(6.7) is used as initial conditions with a small random perturbation in the range of $[-0.01,0.01]$; (ii) then we solve the system numerically for $t=20000$ time units; (iii) compute the euclidean norm between the initially perturbed state and the theoretical one as well as between the final state after $t=20000$ and the theoretical one; (iv) in case the second norm is smaller than the first, meaning that the trajectory approaches the theoretical one-cluster state, we consider the one-cluster state as stable and color the corresponding region in blue, otherwise the state is considered as unstable and the corresponding region is colored in yellow.

The diagrams in the first row of Fig. 6.6 show the influence of the wavenumber on the stability of 
one-clusters. Here, the coupling range is fixed to $P=10$. For adaptively coupled phase oscillators with a global base topology, it has been shown that the stability of rotating-waves of local splay type, i.e., $k \neq 0, N / 2$, does not depend on the wavenumber, see Chapter 4 . However. we observe that in case of a nonlocal base structure the shape of the stability regions crucially depends on the wavenumber $k$, see Fig. $6.6(\mathrm{a}, \mathrm{b}, \mathrm{c})$. Moreover, there are no common regions of stability for the one-cluster states in Fig. 6.6(b,c), i.e., the region of stability in both figures have an empty intersection.

The diagrams in Fig. 6.6(d-f) exemplify the influence of the coupling range on the stability of the rotating-wave cluster with $k=1$. We see that in comparison with Fig. 6.6(a), the regions of stability change significantly. Note further that for $P=25$ the stability regions resemble the results known for the globally coupled base topology, see Chapter 4.

In contrast to the local splay type clusters, the stability regions for the antipodal one-cluster states are the same which can be derived from the following. For antipodal states, the quadratic equation (6.18) simplifies to

$$
\lambda_{l}^{2}-\frac{\lambda_{l}}{2}\left[\left(1-R_{N}^{(l)}\left(\chi_{1}\right)\right) \sin (\beta) \cos (\alpha)-2 \epsilon\right]-\epsilon\left(1-R_{N}^{(l)}\left(\chi_{1}\right)\right) \sin (\alpha+\beta)=0 .
$$

since $L(\alpha, \beta, l, k)=\sin (\alpha+\beta)\left(1-R_{N}^{(l)}\left(\chi_{1}\right)\right)$ for $k=0, N / 2$.

The regions of stability in Fig. 6.6(c) for a nonlocal coupling structure are in agreement with the regions of stability found for a global coupling structure, see Chapter 4 . However, note that Eq. (6.19) differs analytically from the expression found in Corr. 4.2.3. The similarity in the stability regions is only due to the small value of $\epsilon$ and the differences would be more pronounced in the presence of larger $\epsilon$. Note further, that in case of $P=N / 2$, which is equivalent to global coupling, the local order parameter $R^{(l)}$ is either 1 for $l=0$ and 0 otherwise. This agrees with the findings in Chapter 4.

Our stability analysis shows how strongly the ring network structure and confined coupling range alter the stability properties of the clusters. Since the analysis in Appendix A.6 is not restricted to nonlocal coupling structures, it provides the analytic tools to study the influence of more general complex base topologies on the stability of rotating-wave states.

\subsection{The emergence of solitary states}

In this section, we unveil the mechanism behind the formation of solitary states as they are illustrated in Fig. 6.5. As solitary state we define the state where all oscillators in the system are frequency synchronized except one single oscillator, or several oscillators which do not share their local neighborhood. The majority cluster of the synchronized oscillators is also called background cluster.

Let us restrict ourselves to the analysis of a solitary cluster interacting with an in-phase synchronous cluster (see (6.6) and (6.7) with $\chi_{i}=0$ ). Imposing the assumptions, we end-up with the following 4-dimensional model where $\phi$ and $\psi$ describe the dynamics of the solitary cluster 

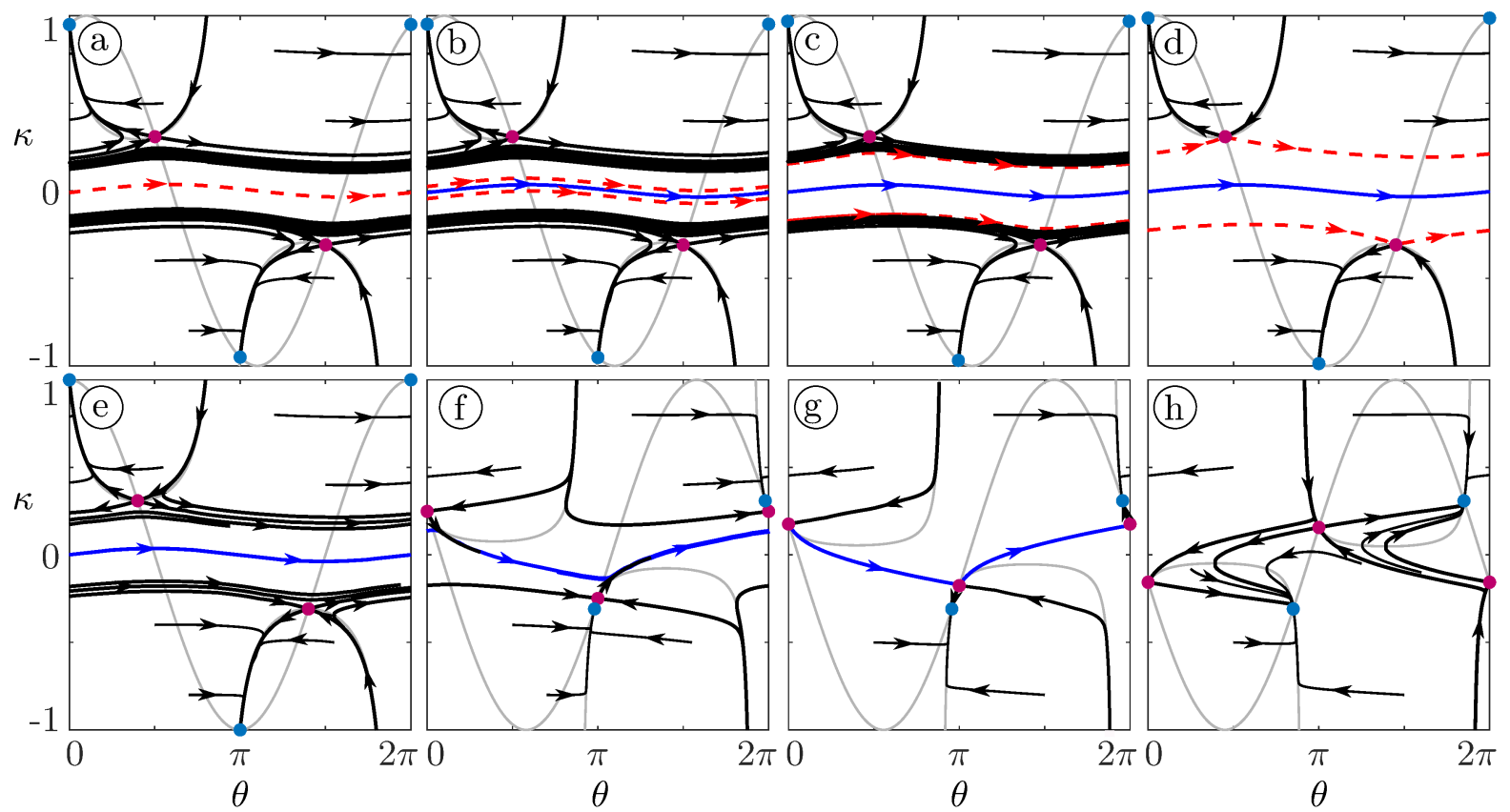

Figure 6.7: Phase portraits for two-dimensional system (6.20)-(6.21). The graphics show the two classes of asymptotic states that are equilibria (colored nodes) and periodic solutions (colored lines). The stability properties of the individual asymptotic states are indicated by the coloring where the blue refers to stable and the red (dashed) to unstable states. In addition, several trajectories are plotted in black including those close to the stable and unstable manifold of the equilibria. The nullclines are displayed as gray lines. For the different panels parameter $\beta$ is varied as shown in Fig. 6.4: (a) $\beta=-0.601 \pi$; (b) $\beta=-0.599 \pi$; (c) $\beta=-0.58 \pi$; (d) $\beta=-0.5515 \pi$; (e) $\beta=-0.5 \pi$; (f) $\beta=-0.08 \pi$; (g) $\beta=-0.0563 \pi$; and (h) $\beta=0.05 \pi$. The other parameters are $\alpha=0.1 \pi$ and $\epsilon=0.01$. Figure modified from [BER20c].

and the background in-phase synchronized cluster, respectively, which are dynamically coupled through $\kappa_{1}$ and $\kappa_{2}$ :

$$
\begin{aligned}
\dot{\phi} & =1-\frac{N-1}{N} \kappa_{1} \sin (\phi-\psi+\alpha), \\
\dot{\psi} & =1+\frac{N-2}{N} \sin \alpha \sin \beta-\frac{1}{N} \kappa_{2} \sin (\phi-\psi-\alpha), \\
\dot{\kappa}_{1} & =-\epsilon\left(\kappa_{1}+\sin (\phi-\psi+\beta)\right), \\
\dot{\kappa}_{2} & =-\epsilon\left(\kappa_{2}-\sin (\phi-\psi-\beta)\right) .
\end{aligned}
$$

The latter equations can be simplified by introducing the phase difference $\theta=\phi-\psi$ as well as by considering a large ensemble of oscillators $(N \rightarrow \infty)$. We obtain the following two-dimensional system for the dynamics of two clusters, one of which is solitary, in the large ensemble limit:

$$
\begin{aligned}
& \dot{\theta}=-\sin \alpha \sin \beta-\kappa \sin (\theta+\alpha), \\
& \dot{\kappa}=-\epsilon(\kappa+\sin (\theta+\beta)),
\end{aligned}
$$

where we denote $\kappa=\kappa_{1}$.

In the following, we study the structure of the phase space of (6.20)-(6.21) for fixed $\alpha=0.1 \pi$ and different values of parameter $\beta$. Several bifurcation scenarios are discovered which give rise to the birth and stability changes of the solitary states. We observe how the stable solitary state emerges in a subcritical pitchfork bifurcation of periodic orbits and disappears in a homoclinic bifurcation with increasing $\beta$. Note that solitary states are given by periodic solutions, where 
the phase difference $\theta(t)$ rotates. Equilibria of this system describe one-cluster states. Figure 6.7 shows several characteristic phase portraits of (6.20)-(6.21) illustrating the bifurcation scenarios with increasing $\beta$ (from (a) to (h)), see also Fig. 6.4.

In Fig. 6.7(a), we observe four equilibria which correspond to certain one-cluster solution. The stable equilibria at $\theta=0$ and $\theta=\pi$ correspond to in-phase synchronous and antipodal where $a_{1}=\pi$ and $a_{i \neq 1}=0$, respectively. The other two saddle equilibria correspond to the special class of double antipodal states, see Chapter 4, and describe therefore phase-cluster similar to those described in [MAI14, TEI19]. While these equilibria can be stable for the reduced system (6.20)-(6.21), they are always unstable for (6.1)-(6.2) in case of global coupling, see Prop. 4.2.4. Additionally, to these four equilibria we find an unstable periodic orbit which corresponds to an unstable solitary state.

With increasing $\beta$, we observe a subcritical pitchfork bifurcation of periodic orbits at $\alpha+\beta=-\pi / 2$ in which the unstable periodic orbit is stabilized and two additional periodic orbits are created. Figure $6.7(\mathrm{~b})$ shows the phase portrait directly after the pitchfork bifurcation. Therefore, we conclude that there exist three solitary states, two of which are unstable and one stable. It is worth to remark that the stability for the reduced system is only necessary but not sufficient to be a stable asymptotic state for the network (6.1)-(6.2).

By increasing $\beta$ even further the basin of attraction of the stable periodic orbit increases and its boundaries are given by the unstable periodic orbits, see Fig. 6.7(c). For $\beta=-0.5515 \pi$, the trajectories of the unstable solitary states merge with the equilibria and become homoclinic orbits of the saddle equilibria (Fig. 6.7(d)). The phase portrait after this homoclinic bifurcation is shown in Fig. 6.7(e).

After the homoclinic bifurcation, with increasing $\beta$, the equilibria are moving towards each other in phase space and exchange their stability in a transcritical bifurcation. This can seen analytically by considering the determining equations for the equilibria $(\theta, \kappa)$ of $(6.20)-(6.21)$

$$
\begin{aligned}
& 0=\cos (\alpha+\beta)-\cos (2 \theta+\alpha+\beta), \\
& \kappa=-\sin (\theta+\beta) .
\end{aligned}
$$

In general, equation (6.22) possesses two solutions for $2 \theta$. At $\alpha+\beta=0, \pi$, however, these two solutions coincide which describes the point of the transcritical bifurcation. The stability of the equilibria can be further computed by considering the two-dimensional system linearized around the equilibria. In a more general setup this has be done Sec. 6.2.2. Figure 6.7(f) displays the phase portrait after the transcritical bifurcation. Remember that although the double antipodal clusters are stable for the reduced system, they are always unstable for the full system, see Prop. 4.2.4.

In Figure 6.7 (g,h) another homoclinic bifurcation is presented in which the stable solitary state becomes a homoclinic orbit of the in-phase and antipodal cluster. The phase portrait close to the homoclinic bifurcation is presented in Fig. 6.7(h). After the homoclinic bifurcation the phase space is divided into the basins of attraction of the two double antipodal states and no more solitary state exist. 


\subsection{Adaptive networks with global base topology versus ring base topology: the differences}

Adaptive networks of coupled phase oscillators have been extensively studied on an all-to-all base structure [AOK09, AOK11, KAS17], see also Chapters 4 and 5. Here, we extend previous work towards more complex base topologies by considering a nonlocal ring base topology on which adaptation takes place. In this section we briefly summarize the main differences resulting from the different base topologies.

For the global base topology, all links between the nodes with the same frequency become active leading to the all-to-all structures within each cluster. Therefore, strongly connected components can be equivalently described by the frequency synchronization of nodes. In contrast to this, for ring networks (also more complex base structures), the frequency synchronization does not necessarily imply connectivity, see Fig. 6.2. As a result, we have adapted the definition of the frequency cluster on a complex base topology as a connected subnetwork with frequency synchronized oscillators.

Another effect induced by the ring base topology concerns the hierarchical ordering of cluster sizes. For global base structures is has been found that a sufficiently large difference of the cluster sizes is necessary for the appearance of multicluster states. In case of a ring structure, the hierarchy is not necessary anymore. In figure 6.3(g-i), we present a five-cluster states that possesses two clusters each of size 7 and additionally two clusters each of size 2. For solitary states this nonhierarchical clustering implies that on a ring structure there can be several solitary nodes (Fig. 6.5(d-f)). A simple explanation for the appearance of the clusters of a similar size is based on the fact that such clusters can be uncoupled in the base coupling structure and, hence, not synchronized. In contrast, in networks with the global base structure, similar clusters tend to be synchronized and merge into one larger cluster.

Regarding the stability of rotating-wave states another striking difference between global and ring base topology is observed. Here the differences are twofold. For a global base topology rotating-waves constitute a $N$-2-dimensional family of solutions with the same collective frequency. On a nonlocal ring, this invariant family is not present anymore and all rotatingwave are different from each other including their frequencies. The same holds true for their stability. While the stability features of all rotating-waves agree on global structures, the stability properties depend crucially on the wavenumber (see Fig. 6.6(a-c)).

\subsection{Summary}

In summary, a model of adaptively coupled identical phase oscillators on a nonlocal ring has been studied. Various frequency synchronized states are observed including one-cluster, multicluster, and solitary states. Those states are similar to those found for a global base topology [KAS17], also Chapters 4 and 5. However, to account for the complex base topology, we have introduced a new definition of one-clusters by means of connected induced subnetworks. This definition allows furthermore to distinguish between multicluster and solitary states in a more strict way than it was done before. 
Since one-cluster states form building blocks for multicluster states, Chapter 5, we have first investigated the existence and stability properties of one-cluster states. Here, we have introduced a novel type of phase-locked states for complex networks, namely local splay states, and have shown that this class of states is nonempty for any nonlocal ring base topology. In particular, we have proved that rotating-wave as well as antipodal states are always phase-locked solutions. Compared with the case of a global base topology, the different clusters of local splay type on a nonlocal ring structure can possess different collective frequencies. In addition, we have proved that local splay cluster are always global splay cluster. This statement relates, therefore, local with global (with respect to "spatial" structures in the network) properties of solutions.

The stability features of rotating-wave states have been studied numerically and analytically. The comparison of both approaches results in a very good agreement. Due to the analytic findings for rotating-wave states on a nonlocal ring, we are able to describe their stability depending on the coupling range $P$ and the wavenumber $k$. The limiting case of global coupling, i.e. $P=N / 2$, is shown to be in agreement with the results presented in Chapter 4.

An interesting feature of the system's behavior are solitary states. They have been previously found to emerge in the Kuramoto-Sakaguchi model with inertia [JAR18]. In this chapter, we show that solitary states are born in a homoclinic bifurcation and can be (de)stabilized in a pitchfork bifurcation of periodic orbits. In order to show this, a two-dimensional effective model is derived governing the dynamics of solitary states. In contrast to the Kuramoto-Sakaguchi model with inertia, we observe a much more complicated bifurcation behavior. In particular, three different solitary states are created due to two individual homoclinic bifurcations. Two of these three solitary states, however, are unstable and bifurcate together with stable solitary states in a subcritical pitchfork bifurcation of periodic orbits.

Our results highlight the delicate interplay between adaptivity and complexity of the network structure. Since this interplay has been rarely investigated from the mathematical viewpoint, so far, this work raises many questions for future research which could be conducted for different network structures beyond nonlocal rings, other dynamical models for the local dynamics, nonidentical units or different adaptation rules. 



\section{Synchronization on adaptive complex network $r$ structures}

In this chapter, we extend the master stability approach to complex dynamical network of $N$ diffusively [PEC98, KEA12, LEH15b] and adaptively coupled oscillators. Moreover, we apply the result to the paradigmatic model of adaptively coupled phase oscillators and provide a novel description for the emergence of multicluster due to the presence of stability islands.

In the following, we consider a network of $N$ diffusively [PEC98, KEA12, LEH15b] and adaptively coupled oscillators of the form

$$
\begin{aligned}
& \dot{\boldsymbol{x}}_{i}=f\left(\boldsymbol{x}_{i}(t)\right)-\sigma \sum_{j=1}^{N} a_{i j} \kappa_{i j} G\left(\boldsymbol{x}_{i}-\boldsymbol{x}_{j}\right), \\
& \dot{\kappa}_{i j}=-\epsilon\left(\kappa_{i j}+a_{i j} \rho H\left(\boldsymbol{x}_{i}-\boldsymbol{x}_{j}\right)\right),
\end{aligned}
$$

where $\boldsymbol{x}_{i}$ is a $d$-dimensional state vector of the $i$ th node with $\boldsymbol{x}_{i} \in \mathbb{C}^{d}, f \in C^{1}\left(\mathbb{C}^{d}, \mathbb{C}^{d}\right)$ describes the local dynamics of node $i$, the function $G \in C^{1}\left(\mathbb{C}^{d}, \mathbb{C}^{d}\right)$ determines the coupling between nodes. The coupling between the nodes is weighted by the dynamical variables $\kappa_{i j} \in \mathbb{R}$ which are adapted according to the function $H \in C^{1}\left(\mathbb{C}^{d}, \mathbb{R}\right)$. The parameter $\sigma \in \mathbb{R}$ is the coupling constant and $\rho \in \mathbb{R}$ is called the adaptation strength. The basic coupling structure is given by the matrix entries $a_{i j} \in\{0,1\}$ of the $N \times N$ adjacency matrix $A$ where a constant row sum $r=\sum_{j=1}^{N} a_{i j}$ for all $i=1, \ldots, N$ is assumed.

Let $s(t)$ be the synchronized state, meaning that $x_{i}=s(t)$ for all $i=1, \ldots, N$. Hence the fully synchronized solution for the equations (7.1)-(7.2) is given by the solution of the following equations

$$
\begin{aligned}
\dot{\boldsymbol{s}} & =f(\boldsymbol{s})+r \sigma \rho H(0) G(0), \\
\kappa^{\mathrm{sync}} & = \begin{cases}-\rho H(0), & \text { if } a_{i j}=1, \\
0, & \text { otherwise }\end{cases}
\end{aligned}
$$

Note that the synchronous solution depends on the row sum if neither $G(0) \neq 0$ nor $H(0) \neq 0$. By scaling $\sigma$ with $1 / r$ this dependency can be omitted.

The chapter is structured as follows. In Section 7.1, we establish the master stability approach for complex adaptive networks of coupled oscillators and prove the main Proposition 7.1.3. This result is then applied to a complex network of adaptively coupled phase oscillators for which the master stability function is completely described in Sec. 7.2. Here, we further show the emergence of stability islands. In Section 7.3, we use the master stability functions to show how multicluster states as well as chimera-like states emergence due to the stability islands. The results of this chapter are summarized in Section 7.4. 


\subsection{The master stability function for adaptive complex networks}

In Ref. [PEC98], the master stability function for dynamical systems of the form (7.1) was introduced. Here, the master stability function is defined as the largest Lyapunov exponent as a function of a complex parameter, the master function parameter, for which eventually the eigenvalues of the Laplacian matrix will be inserted. Once the master stability function is determined, the stability of the synchronous states can be directly deduced for any topology.

In the following we follow and extend the approach of Refs. [PEC98, LEH15b] and present a master stability function which takes adaptation of the coupling weights into account. For this we have to consider the variational equations along the synchronous solution. For convenience we define the following notation

$$
\boldsymbol{a}_{i}=\left(a_{i 1}, \ldots, a_{i N}\right), \quad \operatorname{diag}\left(\boldsymbol{a}_{i}\right)=\left(\begin{array}{ccc}
a_{i 1} & & \\
& \ddots & \\
& & a_{i N}
\end{array}\right)
$$

and the $N \times N^{2}, N^{2} \times N$, and $N^{2} \times N$ matrices

$$
B=\left(\begin{array}{ccc}
\boldsymbol{a}_{1} & & \\
& \ddots & \\
& & \boldsymbol{a}_{N}
\end{array}\right), \quad C=B^{T}-\mathrm{D}, \quad D=\left(\begin{array}{c}
\operatorname{diag}\left(\boldsymbol{a}_{1}\right) \\
\vdots \\
\operatorname{diag}\left(\boldsymbol{a}_{N}\right)
\end{array}\right),
$$

respectively.

Then, the variational equations for system (7.1)-(7.2) along the synchronous solution $s(t)$ can be written in the following vectorized form

$$
\left(\begin{array}{c}
\dot{\xi} \\
\dot{\chi}
\end{array}\right)=\left(\begin{array}{cc}
S & -\sigma B \otimes G(0) \\
-\epsilon \rho C \otimes D H(0) & -\epsilon \mathbb{I}_{N^{2}}
\end{array}\right)\left(\begin{array}{l}
\xi \\
\chi
\end{array}\right),
$$

where $S=\mathbb{I}_{N} \otimes D f(s)+\sigma \rho H(0)(L(A) \otimes D G(0)), \boldsymbol{\xi}=\boldsymbol{x}-\mathbb{I}_{N} \otimes \boldsymbol{s}$, and $\boldsymbol{\chi}=\boldsymbol{\kappa}-\boldsymbol{\kappa}^{\text {sync }}$ with Kronecker product denoted by $\otimes$. Further, $x \in \mathbb{C}^{N \cdot d}$ is the system state vector where all individual nodal states are stacked on each other ordered by the node index, i.e., $\boldsymbol{x}=\left(\boldsymbol{x}_{1}, \ldots, \boldsymbol{x}_{N}\right)^{T}$. Analogously, $\boldsymbol{\kappa}=\left(\kappa_{11}, \ldots, \kappa_{1 N}, \ldots, \kappa_{N N}\right)$. Further, the Laplacian $L(A)$ for the corresponding adjacency matrix $A$ is given by

$$
L(A)=\mathscr{D}(A)-A,
$$

where

$$
\mathscr{D}(A)=\left(\begin{array}{lll}
\sum_{j=1}^{N} a_{1 j} & & \\
& \ddots & \\
& & \sum_{j=1}^{N} a_{N j}
\end{array}\right)
$$

see (2.3). 
In the following Lemma, we summarize a few simple relations which are used to simply calculation, later on.

Lemma 7.1.1 Let $B, C$, and $D$ given as above. Then the following relations hold true:

1. $B \cdot B^{T}=r \mathbb{I}_{N}$,

2. $B \cdot D=A$,

3. $B \cdot C=L(A)$,

4. $C^{T} C=L(A)+L\left(A^{T}\right)$.

Proof. The results are proved by direct calculation.

1.

$$
B \cdot B^{T}=\left(\begin{array}{ccc}
\boldsymbol{a}_{1} & & \\
& \ddots & \\
& & \boldsymbol{a}_{N}
\end{array}\right)\left(\begin{array}{ccc}
\boldsymbol{a}_{1}^{T} & & \\
& \ddots & \\
& & \boldsymbol{a}_{N}^{T}
\end{array}\right)=\left(\begin{array}{ccc}
\sum_{j=1}^{N} a_{1 j} a_{1 j} & & \\
& \ddots & \\
& & \sum_{j=1}^{N} a_{N j} a_{N j}
\end{array}\right) .
$$

2.

$$
B \cdot D=\left(\begin{array}{ccc}
\boldsymbol{a}_{1} & & \\
& \ddots & \\
& & \boldsymbol{a}_{N}
\end{array}\right)\left(\begin{array}{c}
\operatorname{diag}\left(\boldsymbol{a}_{1}\right) \\
\vdots \\
\operatorname{diag}\left(\boldsymbol{a}_{N}\right)
\end{array}\right)=\left(\begin{array}{c}
\boldsymbol{a}_{1} \operatorname{diag}\left(\boldsymbol{a}_{1}\right) \\
\vdots \\
\boldsymbol{a}_{N} \operatorname{diag}\left(\boldsymbol{a}_{N}\right)
\end{array}\right)=\left(\begin{array}{ccc}
a_{11} a_{11} & \cdots & a_{1 N} a_{1 N} \\
\vdots & \ddots & \vdots \\
a_{N 1} a_{N 1} & \cdots & a_{N N} a_{N N}
\end{array}\right) .
$$

3. Using the results from 1 . and 2., we get

$$
B \cdot C=B \cdot B^{T}-B \cdot D=L(A) .
$$

4. By applying 1. and 2., we find

$$
\begin{aligned}
C^{T} \cdot C & =\left(B-D^{T}\right) \cdot\left(B^{T}-D\right)=r \mathbb{I}_{N}-A-(B \cdot D)^{T}+\sum_{j=1}^{N} \operatorname{diag}\left(\boldsymbol{a}_{j}\right)^{2} \\
& =r \mathbb{I}_{N}-A-A^{T}+\mathscr{D}\left(A^{T}\right) .
\end{aligned}
$$

It was shown in Chapter 4 and 6 that due to the structure of the variational equations, there are locally always degrees of freedom corresponding to the $N^{2}-N$ eigenvalues $\lambda=-\epsilon$. In order to reduce the equations (7.5), we are studying the corresponding eigenspace. Consider the equation

$$
\left(\begin{array}{cc}
S-\epsilon \mathbb{I}_{N d} & -\sigma B \otimes G(0) \\
-\epsilon \rho C \otimes D H(0) & 0
\end{array}\right)\left(\begin{array}{l}
\xi \\
\chi
\end{array}\right)=0 .
$$

From $C \otimes D H(0) \xi=0$, it can be immediately deduced that $\xi_{i}=\bar{\xi} \in \mathbb{C}^{d}$ for all $i=1, \ldots, N$. Assuming that $\xi=0$ would lead to $B \chi=0$ as the defining equation for the eigenvectors. In fact, the following results show that $B \chi=0$ suffices to find $N^{2}-N$ many linearly independent vectors spanning the eigenspace. The eigenvectors themselves are additionally independent of time. 
Lemma 7.1.2 Let $\chi=\left(\chi_{1}^{T}, \ldots, \chi_{N}^{T}\right)^{T}$ with $\chi_{i}^{T}=\left(\left(\chi_{i}\right)_{1}, \ldots,\left(\chi_{i}\right)_{N}\right)$ for all $i \in\{1, \ldots, N\}$, then the following two relations hold true

1. $\operatorname{ker}(B)=\left\{\chi \in \mathbb{R}^{N^{2}}: \sum_{j=1}^{N} a_{i j}\left(\chi_{i}\right)_{j}=0 \forall i \in\{1, \ldots, N\}\right\}$

2. $\operatorname{ker}\left(D^{T}\right)=\left\{\chi \in \mathbb{R}^{N^{2}}: \sum_{j=1}^{N} a_{j i}\left(\chi_{j}\right)_{i}=0 \forall i \in\{1, \ldots, N\}\right\}$.

Further, $\operatorname{dim}(\operatorname{ker}(B))=\operatorname{dim}\left(\operatorname{ker}\left(D^{T}\right)\right)=N^{2}-N$.

Proof. 1. We find by direct calculation

$$
B \chi=\left(\begin{array}{c}
\boldsymbol{a}_{1} \chi_{1} \\
\vdots \\
\boldsymbol{a}_{N} \chi_{N}
\end{array}\right)=\left(\begin{array}{c}
\sum_{j=1}^{N} a_{1 j}\left(\chi_{1}\right)_{j} \\
\vdots \\
\sum_{j=1}^{N} a_{N j}\left(\chi_{N}\right)_{j}
\end{array}\right)
$$

2.

$$
\chi^{T} D=\left(\chi_{1}^{T}, \ldots, \chi_{N}^{T}\right)\left(\begin{array}{c}
\operatorname{diag}\left(\boldsymbol{a}_{1}\right) \\
\vdots \\
\operatorname{diag}\left(\boldsymbol{a}_{N}\right)
\end{array}\right)=\sum_{j=1}^{N} \chi_{j}^{T} \boldsymbol{a}_{j}=\sum_{j=1}^{N}\left(\left(\chi_{j}\right)_{1} a_{j 1}, \ldots,\left(\chi_{j}\right)_{N} a_{j N}\right)
$$

It is easy to verify that $\operatorname{rank}(B)=\operatorname{rank}\left(D^{T}\right)=N$ and $\operatorname{dim}(\operatorname{ker}(B))=\operatorname{dim}\left(\operatorname{ker}\left(D^{T}\right)\right)=N^{2}-N$.

With this result we are able to find a proper transformation in order to determine the master stability function for adaptively coupled dynamical systems.

Proposition 7.1.3 Let (7.1)-(7.2) possess a synchronous state given by (7.3)-(7.4). Further, let (7.5) be the variational equations around this synchronous solution and assume that the Laplacian matrix $L(A)$ is diagonalizable. Then, the synchronous solution is stable if and only if for all eigenvalues $\mu \in \mathbb{C}$ of the Laplacian matrix the largest Lyapunov exponent of the following differential equations is smaller than zero.

$$
\begin{aligned}
& \frac{\mathrm{d} \zeta}{\mathrm{d} t}=(D f(s)+\sigma \rho \mu H(0) D G(0)) \zeta-\sigma G(0) \kappa \\
& \frac{\mathrm{d} \kappa}{\mathrm{d} t}=-\epsilon(\rho \mu \mathrm{DH}(0) \zeta+\kappa)
\end{aligned}
$$

Here, $\zeta \in \mathbb{C}^{d}$ and $\kappa \in \mathbb{C}$.

Proof. Due to Lemma 7.1.2 there are $N^{2}-N$ independent vectors $\boldsymbol{w}_{l}\left(l=1, \ldots, N^{2}-N\right)$ spanning the kernel of $B$. Using the Gram-Schmidt procedure we find an ortho-normal basis for $\operatorname{ker}(B)=$ $\operatorname{span}\left\{\boldsymbol{v}_{1}, \ldots, \boldsymbol{v}_{N^{2}-N}\right\}$. With this we define the $N^{2} \times\left(N^{2}-N\right)$ matrix $Q=\left(\boldsymbol{v}_{1}, \ldots, \boldsymbol{v}_{N^{2}-N}\right)$. Consider now the $\left(N^{2}+N d\right) \times\left(N^{2}+N d\right)$ matrix

$$
R=\left(\begin{array}{ccc}
\mathbb{I}_{N d} & 0 & 0 \\
0 & (1 / r) B^{T} & Q
\end{array}\right)
$$


with left inverse

$$
R^{-1}=\left(\begin{array}{cc}
\mathbb{I}_{N d} & 0 \\
0 & B \\
0 & Q^{T}
\end{array}\right)
$$

i.e., $R^{-1} R=\mathbb{I}_{N^{2}+N d}$. Introduce the new coordinates given by $R\left(\begin{array}{l}\hat{\xi} \\ \hat{\chi}\end{array}\right)=\left(\begin{array}{l}\xi \\ \chi\end{array}\right)$ for which the variational equations (7.5) read

$$
\frac{\mathrm{d}}{\mathrm{d} t}\left(\begin{array}{l}
\hat{\boldsymbol{\xi}} \\
\hat{\chi}
\end{array}\right)=R^{-1}\left(\begin{array}{cc}
S & -\sigma B \otimes G(0) \\
-\epsilon \rho C \otimes D H(0) & -\epsilon \mathbb{I}_{N^{2}}
\end{array}\right) R\left(\begin{array}{l}
\hat{\xi} \\
\hat{\chi}
\end{array}\right) .
$$

We further observe

$$
\begin{aligned}
R^{-1}\left(\begin{array}{cc}
S & -\sigma B \otimes G(0) \\
-\epsilon \rho C \otimes D H(0) & -\epsilon \mathbb{I}_{N^{2}}
\end{array}\right) R & =R^{-1}\left(\begin{array}{ccc}
S & -\sigma \mathbb{I}_{N} \otimes G(0) & 0 \\
-\epsilon \rho C \otimes D H(0) & -\epsilon / r B^{T} & -\epsilon Q
\end{array}\right) \\
& =\left(\begin{array}{ccc}
S & -\sigma \mathbb{I}_{N} \otimes G(0) & 0 \\
-\epsilon \rho L(A) \otimes D H(0) & -\epsilon \mathbb{I}_{N} & 0 \\
-\epsilon \rho Q^{T} C \otimes D H(0) & 0 & -\epsilon \mathbb{I}_{N^{2}-N}
\end{array}\right) .
\end{aligned}
$$

These equations yield that there are the $N d+N$ many coupled differential equations left

$$
\frac{\mathrm{d}}{\mathrm{d} t}\left(\begin{array}{c}
\hat{\xi} \\
\tilde{\chi}
\end{array}\right)=\left(\begin{array}{cc}
S & -\sigma \mathbb{I}_{N} \otimes G(0) \\
-\epsilon \rho L(A) \otimes D H(0) & -\epsilon \mathbb{I}_{N}
\end{array}\right)\left(\begin{array}{c}
\hat{\xi} \\
\tilde{\chi}
\end{array}\right)
$$

with $\tilde{\chi}=\hat{\chi}_{1}$ that determine the stability for the synchronous state and $N^{2}-N$ equations

$$
\frac{\mathrm{d}}{\mathrm{d} t} \bar{\chi}=\left(\begin{array}{lll}
-\epsilon \rho Q^{T} C \otimes D H(0) & 0 & -\epsilon \mathbb{I}_{N^{2}-N}
\end{array}\right)\left(\begin{array}{c}
\hat{\xi} \\
\tilde{\chi} \\
\bar{\chi}
\end{array}\right)
$$

with $\bar{\chi}=\left(\hat{\chi}_{2}^{T}, \ldots, \hat{\chi}_{N}^{T}\right)^{T}$ which are unidirectionally coupled to the variables $\hat{\xi}$ and $\tilde{\chi}$ and can be solved explicitly once the latter once are known. By assumption, there is a unitary matrix $D_{L(A)}=U^{H} L(A) U$ where $D_{L(A)}$ is the diagonalization of the Laplacian matrix $L(A)$. Transforming the differential equations (7.9) by using the unitary transformation $U$, we get

$$
\frac{\mathrm{d}}{\mathrm{d} t}\left(\begin{array}{l}
\zeta \\
\kappa
\end{array}\right)=\left(\begin{array}{cc}
\mathbb{I}_{N} \otimes D f(\boldsymbol{s})+\sigma \rho H(0)\left(D_{L(A)} \otimes D G(0)\right) & -\sigma \mathbb{I}_{N} \otimes G(0) \\
-\epsilon \rho D_{L(A)} \otimes D H(0) & -\epsilon \mathbb{I}_{N}
\end{array}\right)\left(\begin{array}{l}
\zeta \\
\kappa
\end{array}\right)
$$

where $\left(\begin{array}{cc}U \otimes \mathbb{I}_{d} & 0 \\ 0 & U\end{array}\right)\left(\begin{array}{l}\hat{\xi} \\ \tilde{\chi}\end{array}\right)=\left(\begin{array}{l}\zeta \\ \kappa\end{array}\right)$.

By using the result in Proposition 7.1.3, the master stability function can be determined for any diffusive dynamical system on a adaptive network. Note that Eq. (7.8) is explicitly solvable. the 
solutions reads

$$
\kappa=\kappa_{0} e^{-\epsilon\left(t-t_{0}\right)}-\epsilon \rho \mu \mathrm{DH}(0) \int_{t_{0}}^{t} e^{-\epsilon\left(t-t^{\prime}\right)} \zeta\left(t^{\prime}\right) \mathrm{d} t^{\prime} .
$$

Here, the first term vanishes as $t \rightarrow \infty$. Hence, we can neglect it in order to study the asymptotic dynamics of (7.7)-(7.8) which are rewritten in integro-differential form (as $t \rightarrow \infty$ )

$$
\frac{\mathrm{d} \zeta}{\mathrm{d} t}=\mathrm{D} f(s) \zeta+\tilde{\mu}\left(H(0) \mathrm{D} G(0) \zeta+\epsilon \mathrm{DH}(0) G(0) \int_{t_{0}}^{t} e^{-\epsilon\left(t-t^{\prime}\right)} \zeta\left(t^{\prime}\right) \mathrm{d} t^{\prime}\right)
$$

with master function parameter $\tilde{\mu}=\rho \sigma \mu$. We apply this result in the subsequent sections and connect it to our understanding of the stability of multicluster states.

\subsection{Stability islands in the presence of adaptation}

In this section, we apply the general result on the master stability function for complex adaptive networks, as it was derived in the previous section. For this, we use a phase oscillator model. Note, however, that the master stability approach presented above is not restricted to this kind of networks.

In analogy with Chapter 6, we consider the following adaptive network of $N$ coupled phase oscillators

$$
\begin{aligned}
\frac{\mathrm{d} \phi_{i}}{\mathrm{~d} t} & =1-\sigma \sum_{j=1}^{N} a_{i j} \kappa_{i j} \sin \left(\phi_{i}-\phi_{j}+\alpha\right) \\
\frac{\mathrm{d} \kappa_{i j}}{\mathrm{~d} t} & =-\epsilon\left(\kappa_{i j}+a_{i j} \sin \left(\phi_{i}-\phi_{j}+\beta\right)\right) .
\end{aligned}
$$

Let us further consider all networks with constant row-sum, i.e., $r=\sum_{j=1}^{N} a_{i j}$ for all $i=1, \ldots, N$. Then the synchronous solution of (7.11)-(7.12) is given by

$$
\begin{aligned}
& \phi^{\mathrm{sync}}=(1-r \sigma \sin (\alpha)) t \\
& \kappa^{\mathrm{sync}}=-\sin (\beta) .
\end{aligned}
$$

Note that this sort of solutions have been already exhaustively analyzed for the case of a globally coupled network in Chapter 4. The synchronous state (7.13)-(7.14) is an equilibrium relative to the shift symmetry of (7.11)-(7.12). Thus, the synchronous state is independent of the value $r$.

In order to analyze the stability of the synchronous states (7.13)-(7.14), we have to linearize the equations (7.11)-(7.12) around these states. Using the result in Proposition 7.1.3, the stability of the synchronous solution is governed by the two dimensional set of equations

$$
\frac{\mathrm{d}}{\mathrm{d} t}\left(\begin{array}{l}
\zeta \\
\kappa
\end{array}\right)=\left(\begin{array}{cc}
\mu \sigma \cos (\alpha) \sin (\beta) & -\sigma \sin (\alpha) \\
-\epsilon \mu \cos (\beta) & -\epsilon
\end{array}\right)\left(\begin{array}{l}
\zeta \\
\kappa
\end{array}\right),
$$

where $\mu \in \mathbb{C}$ is an eigenvalue of the Laplacian matrix $L$ corresponding to the base network described by the adjacency matrix $A$. The characteristic polynomial in $\lambda$ of the latter system is of 
degree two and reads

$$
\lambda^{2}+(\epsilon-\sigma \mu \cos (\alpha) \sin (\beta)) \lambda-\epsilon \sigma \mu \sin (\alpha+\beta)=0 .
$$

Note that for $\mu=0$ the eigenvalues are $\lambda=-\epsilon$ and $\lambda=0$ which corresponds to the shift symmetry. With this, the master stability function for the equations (7.11)-(7.12) is given by $\left.\max \left(\mathfrak{R}\left(\lambda_{1}(\tilde{\mu})\right)\right), \mathfrak{R}\left(\lambda_{2}(\tilde{\mu})\right)\right)$ where $\lambda_{1,2}$ solve Eq. (7.15) for a given set of parameters $\alpha, \beta$ and $\epsilon$. Here, the master function parameter is given by $\tilde{\mu}=\sigma \mu$.

Corollary 7.2.1 Let (7.13)-(7.14) be the synchronous solution for the equations (7.11)-(7.12) with $\sigma>0$. Further, assume the Laplacian matrix $L(A)$ is symmetric, i.e., the network is undirected. Then the synchronous solution is stable if the parameters $\alpha$ and $\beta$ are from following set

$$
\left(\{-\pi<\alpha+\beta<0\} \bigcap\left\{-\frac{\pi}{2}<\alpha<\frac{\pi}{2},-\pi<\beta<0\right\} \bigcap\left\{\frac{\pi}{2}<\alpha<\frac{3 \pi}{2}, 0<\beta<\pi\right\}\right) \bmod 2 \pi .
$$

Proof. Since $L(A)$ is symmetric it is diagonalizable and has only positive real eigenvalues. As shown above, we are allowed to use Proposition 7.1.3. From equation (7.15), we find that the eigenvalues $\lambda$ are strictly negative if and only if $\sin (\alpha+\beta)<0$ and $(\epsilon-\sigma \mu \cos (\alpha) \sin (\beta))>0$. The first of the latter inequalities implies the neccessary condition for stability $-\pi<\alpha+\beta<0$. The other inequality yields

$$
\frac{\epsilon}{\sigma \mu}>\cos (\alpha) \sin (\beta)
$$

Since $\epsilon, \mu, \sigma>0$, the latter inequality implies the statement of the corollary.

In corollary 7.2.1, a relation between stability and the parameters $\alpha$ and $\beta$ is established for undirected networks. In case of directed networks, the Laplacian eigenvalue might be complex, and hence the stability cannot be immediately deduced from the Eq. (7.15). Figure 7.1 displays the master stability function determined for different values for the parameter of $\beta$. Here, we fix $\alpha$ and $\epsilon$. The blue colored areas correspond to master function parameter $\tilde{\mu}=\sigma \mu$, where $\mu$ is an eigenvalues o the Laplacian, that would lead to stable dynamics. By changing the control parameter $\beta$ various shapes of the stable regions are visible.

We, first, observe that the master stability function in all cases is symmetric with respect to the real axis $(\mathfrak{J}(\tilde{\mu})=0)$. This symmetry stems from the fact that if $\lambda_{1,2}$ solve (7.15) for $\mu$ then $\bar{\lambda}_{1,2}$ solve (7.15) for $\bar{\mu}$ where the overline indicates the complex conjugate. Secondly, form some parameter, e.g. Fig. 7.1 (a,d,e), almost the whole half-space from the left and right of the imaginary axis belong to the stable regime. Note that in case of no adaptation the stability of the synchronous solution is solely described by the $\cos (\alpha)$ which is then scaled by $\tilde{\mu} \sin (\beta)$. As a third observation, we find parameters where almost all regions of the $\tilde{\mu}$ parameter space correspond to unstable dynamics, e.g. Fig. 7.1(b,c,f). However, there exist small islands, i.e., bounded regions in $\tilde{\mu}$ parameter space, that correspond to stable dynamics. To understand the emergence of these islands, we further anylze the boundary that separates the stable $\left(\mathfrak{R}\left(\lambda_{1,2}\right)>0\right)$ from the unstable region $\left(\mathfrak{R}\left(\lambda_{1,2}\right)>0\right)$. 

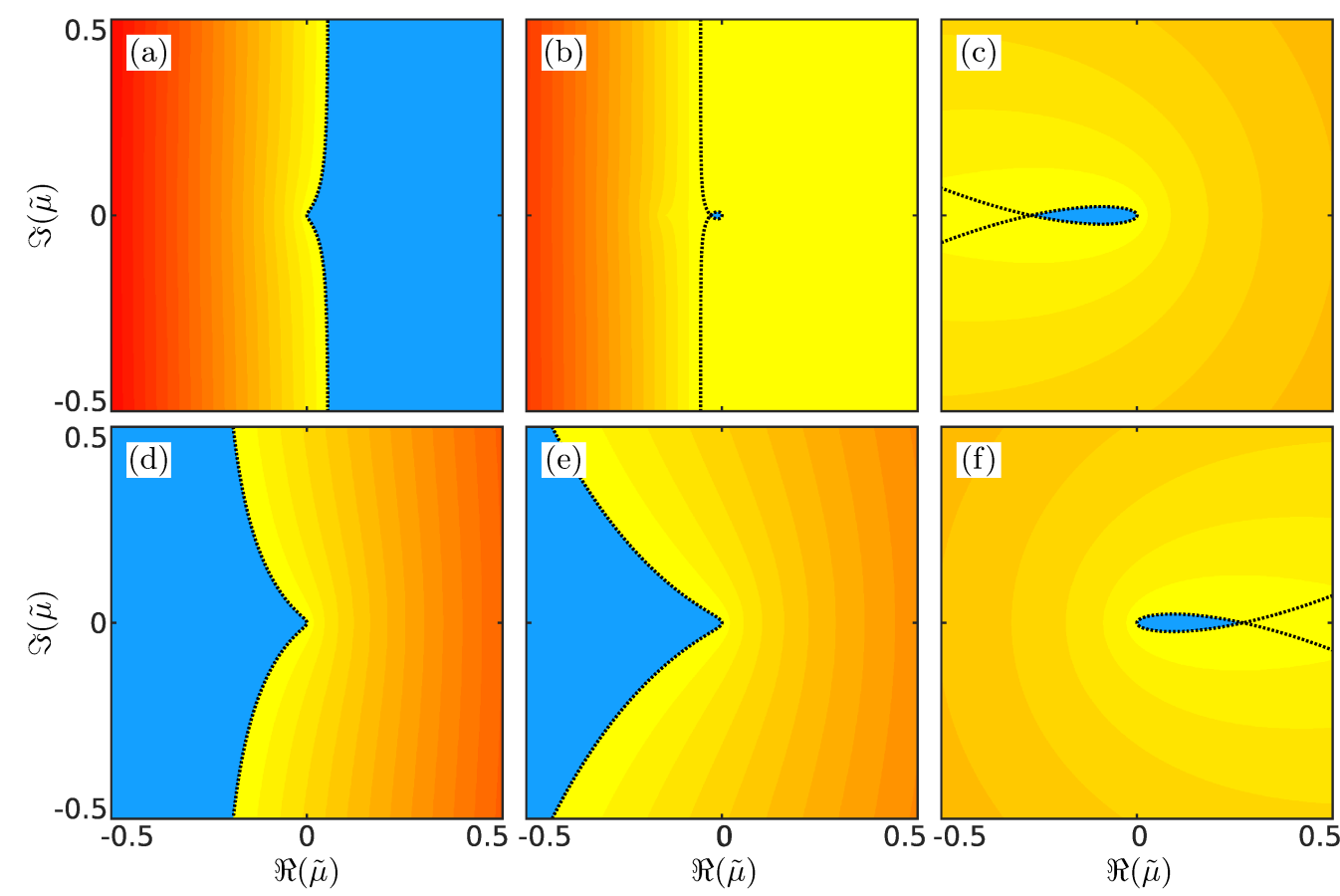

$\max (\Re(\lambda)$

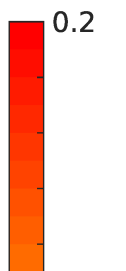

Figure 7.1: The figure shows the master stability function for the equations (7.11)-(7.12). Regions belonging to negative Lyapunov exponents are colored blue. The one dimensional curve where at least one eigenvalue of (7.15) has zero real part is given as a black dotted line. Parameter: (a) $\beta=-0.8 \pi$, (b) $\beta=-0.2 \pi$, (c) $\beta=-0.02 \pi$, (d) $\beta=0.05 \pi$, (e) $\beta=0.1 \pi$, and (f) $\beta=0.98 \pi$. The other parameters are $\alpha=0.3 \pi$ and $\epsilon=0.01$.

In order to describe the boundaries between regions in $\tilde{\mu}$ parameter space that would lead to stable local dynamics or regions that would lead to unstable local dynamics, we consider $\lambda=\mathrm{i} \gamma$. Plugging this into the equation (7.15) we obtain the following parameterized expression for the boundary

$$
\tilde{\mu}(\tilde{\gamma})=\epsilon \frac{\left.-\sin (\alpha) \cos (\beta) \tilde{\gamma}^{2}+\mathrm{i}\left(\sin (\alpha+\beta) \tilde{\gamma}+\cos (\alpha) \sin (\beta) \tilde{\gamma}^{3}\right)\right)}{\sin ^{2}(\alpha+\beta)+\cos ^{2}(\alpha) \sin ^{2}(\beta) \tilde{\gamma}^{2}}
$$

where we use $\tilde{\gamma}=\gamma / \epsilon$. The curves given by the latter parametrization of the boundary are displayed in Fig. 7.1 as dotted lines and agree very well with the actual borders. We further notice that the emergence of the stability islands is a direct consequence of the presence of adaptation. This can be seen by using $\lambda=\mathrm{i} \gamma$ and Eq. (7.15) where we put $\epsilon=0$. This ansatz results in the simple linear boundary equation $\tilde{\mu}=\mathrm{i} \gamma /(\cos (\alpha) \sin (\beta))$ which does not give rise to stability islands. Due to the symmetry of the master stability function, a necessary condition to observe find a stability island is that the curve $\tilde{\mu}(\tilde{\gamma})$ possesses two crossings with the real axis. From Eq. 7.15, we thus deduce the following condition

Corollary 7.2.2 The master stability function of (7.11)-(7.12) possesses stability islands if and only if

$$
\frac{\sin (\alpha+\beta)}{\cos (\alpha) \sin (\beta)}<0
$$

The presence of stability islands is a very intriguing and unexpected effect introduced by adaptation. In the following section the impact of stability islands on the formation of multicluster is described. 


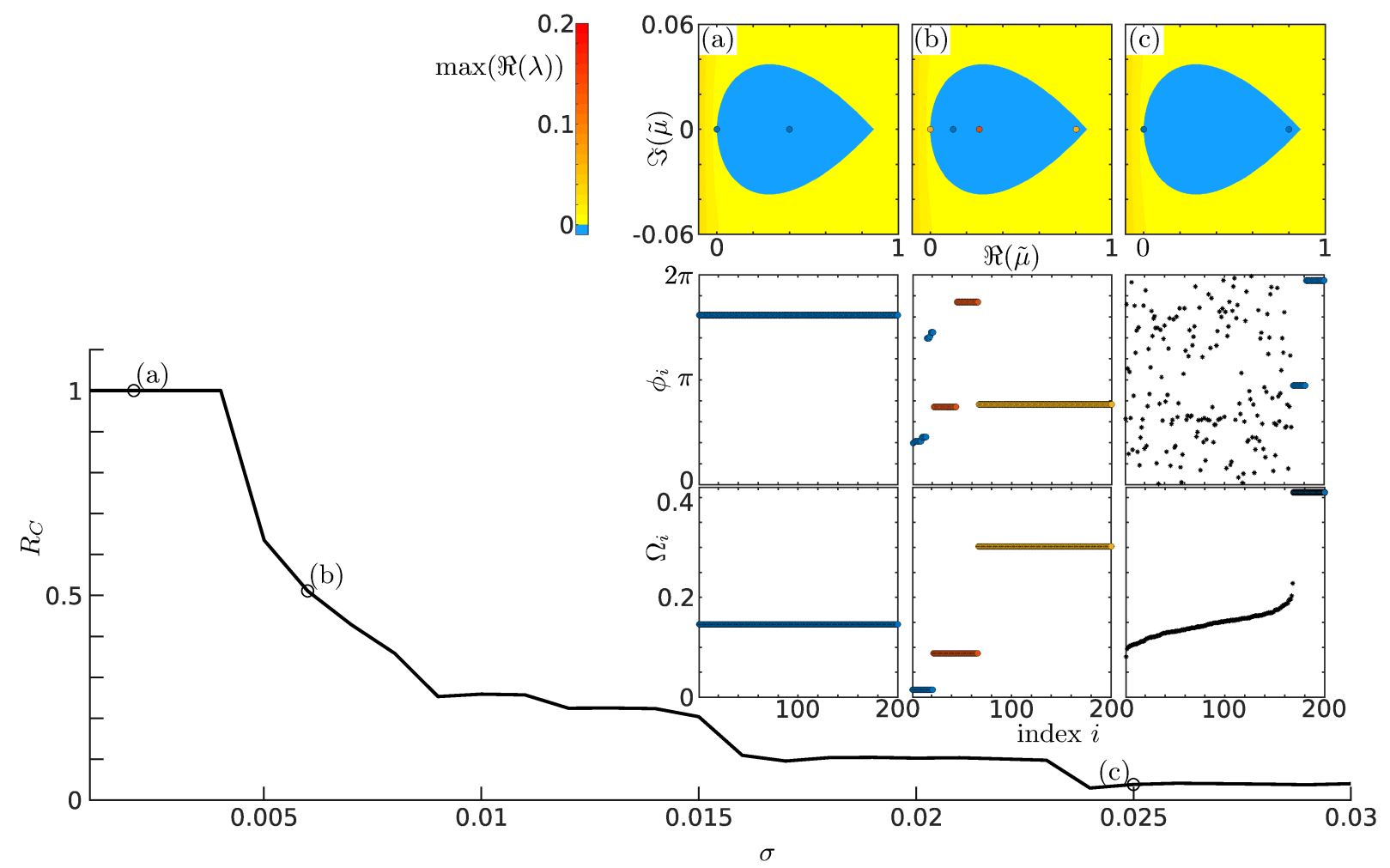

Figure 7.2: Results for an adiabatic continuation in the coupling constant $\sigma$ of the full synchronous solution $\phi_{i}=0$ and $\kappa_{i j}=-\sin (\beta)$ for a globally coupled network of $N=200$ oscillators (7.11)-(7.12). The cluster parameter $R_{C}$ for different values of $\sigma$ is presented. As an inset, for the three values (a) $\sigma=0.002$, (b) $\sigma=0.006$, and (c) $\sigma=0.025$, the master stability function, the phases $\phi_{i}$ of the final state, and the frequencies of the oscillators $\Omega_{i}$ are plotted. The oscillators are sorted as in Fig 5.2. If more than or equal to ten (for numerical convenience) oscillators have the same frequency (coherent groups) all nodes of this group are plotted as circles and with a respective color. All other oscillators are plotted as an asterisk. The master function parameter $\tilde{\mu}=\sigma \mu$ for the subnetworks induced by the coherent groups are plotted together with the master stability function. The colors of each coherent group agree in all three plots. Parameters: $\alpha=0.49 \pi, \beta=0.88 \pi, \epsilon=0.01$.

\subsection{Stability islands and implications for the emergence of multicluster states}

In this section, we explore the relation between the stability islands and the formation of multiclusters. In order to do this, we perform the following numerical analysis. We choose parameters $\alpha$ and $\beta$ such that there exists a stability island. Then we prepare the initial condition to be an in-phase synchronous state, i.e., $\phi_{i}=0$ and $\kappa_{i j}=-\sin (\beta)$ and integrate numerically the system (7.11)-(7.12) with $N=200$ oscillators for $t=30000$. Thus, we perform an adiabatic continuation starting from $\sigma=0.001$ and ending at $\sigma=0.03$. Note that $\sigma=1 / N=0.005$ lies within this range. In order to illustrate the impact of the stability island on the dynamics, we perform this analysis for a globally coupled as well as for a complex network. The complex network which we have chosen for the subsequent analysis is connected, directed and has row sum $r=50$. An illustration of the adjacency matrix can be found in the Appendix A.8.

Figure 7.2 shows the cluster parameter $R_{C}$, see (2.18) in Chapter 2, for different values of the coupling constant $\sigma$. Note that $R_{C}=1$ refers to full in-phase synchrony of the oscillators. We observe that for small $\sigma$ the synchronous solution is stable, see Fig. 7.2(a). For the sake of 
simplicity, the coupling matrix $\kappa_{i j}$ is not displayed for all examples Fig. 7.2(a-c). Here, the stability of the synchronous state is directly implied by the master stability function. We note that all Laplacian eigenvalues $\mu$ of a globally coupled network are given by $\mu=0$ and $\mu=N$. In Fig. 7.2(a), all master function parameters $\sigma \mu$ lie within the stability islands.

By increasing the coupling constant, the master function parameter gets pushed out of the regions of stability and the synchronous states becomes unstable. For intermediate values of $\sigma$ the emergence of multiclusters with hierachical structure in the cluster size is observed, see also Chapter 5. In Fig. 7.2(b) a multicluster states is shown with three clusters. For numerical convenience, we only count groups of oscillators with more than or equal to 10 members as a cluster. Note that for the systems (7.11)-(7.12) in-phase synchronous and antipodal clusters have the same properties. In Chapter 5, the role of the hierarchical structure of the cluster sizes have been discussed. We have found that due to the frequency difference the coupling between the clusters vanish on average. Hence we argued that the stability is effectively described by the stability of the one-clusters of which the multicluster consists, see Section 5.6. Here, we follow this argument and consider the subnetworks induced by the groups of nodes (oscillators) with the frequency. In the case of global coupling each of the subnetworks is globally coupled, as well. The individual master function parameter for the induced subnetworks of the three clusters are plotted together with the master stability function. For all three subnetworks the master function parameters lie in the stable region and thus the corresponding one-clusters are stable and hence the multicluster. This formation of a multicluster is remarkable and is well explained by the master stability function. In addition it is in very good agreement with the numerical analysis in Ref. [KAS17].

Increasing the coupling constant further shows the emergence of incoherence. In Figure 7.2(c), we show the coexistence of a coherent and an incoherent cluster. These states, also called chimeralike states, have been numerically analyzed in Refs. [KAS17, KAS18, KAS18a]. Surprisingly, also for these states, the stability of the coherent cluster is determined by the master function parameter corresponding to the subnetwork induced by the coherent nodes. This fact underlines, once more, the necessity of the building block approach outlined in Chapters 4 and 5 .

In the following we show that the results obtained for the global network translate to set-ups with complex network structure. In Figure 7.3, again, the cluster parameter $R_{C}$ for different values of the coupling constant $\sigma$ is presented. As in the case of global coupling, we observe that the synchronous state is stable for small values of the coupling constant. After the destabilization of the synchronous state, the emergence of a multicluster is shown, see Fig. 7.3(b). In contrast to the globally coupled case, the coherent groups do not necessarily induce subnetworks with a constant row sum. Hence, clusters with slightly perturbed phase distribution occur. However, the stability of the single clusters is again well described by the master stability function since all master function parameters for the individual clusters lie within the stability region. The same holds true for the state presented in Fig. 7.3(c). Here, we observe the coexistence of an incoherent and a coherent cluster. The stability of the latter is given by the master stability function. 


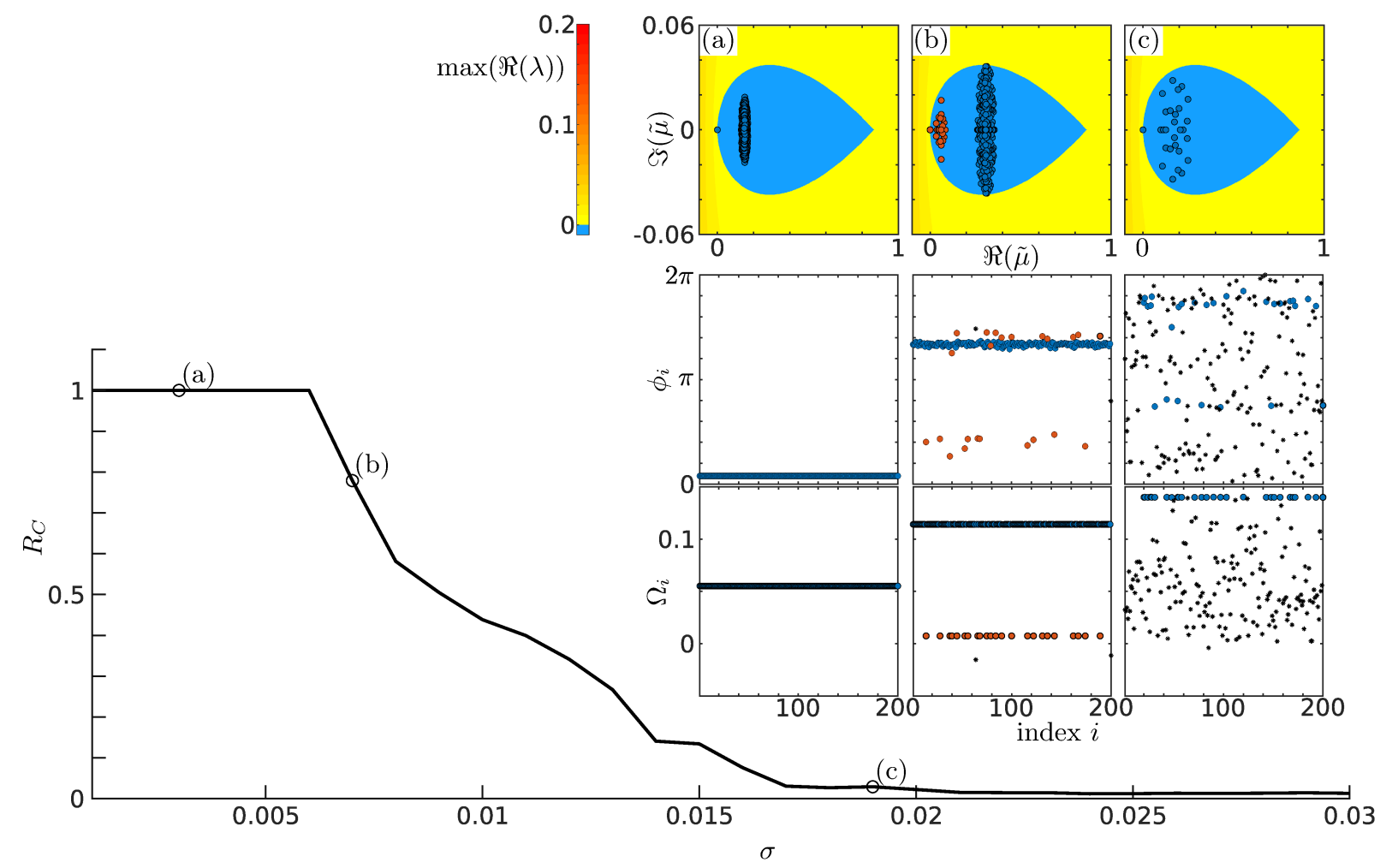

Figure 7.3: Results for an adiabatic continuation in the coupling constant $\sigma$ of the full synchronous solution $\phi_{i}=0$ and $\kappa_{i j}=-\sin (\beta)$ for a complex network (see Appendix A.8) of $N=200$ oscillators (7.11) $-(7.12)$. The cluster parameter $R_{C}$ for different values of $\sigma$ is presented. As an inset, for the three values (a) $\sigma=0.003$, (b) $\sigma=0.007$, and (c) $\sigma=0.019$, the master stability function, the phases $\phi_{i}$ of the final state, and the frequencies of the oscillators $\Omega_{i}$ are plotted. If more than or equal to ten ()for numerical convenience) oscillators have the same frequency (coherent groups) all nodes of this group are plotted as circles and with a respective color. All other oscillators are plotted as an asterisk. The master function parameter $\tilde{\mu}=\sigma \mu$ for the subnetworks induced by the coherent groups are plotted together with the master stability function. The colors of each coherent group agree in all three plots. Parameters: $\alpha=0.49 \pi$, $\beta=0.88 \pi, \epsilon=0.01$.

\subsection{Summary}

In this chapter, we have studied the stability of the synchronous solution for a complex network of adaptively coupled oscillators. For this, the well-known master stability approach is generalized to networks with adaptive and hence time evolving coupling weights. The master stability approach is already well established and was generalized for various applications, e.g., to understand the stability of cluster synchronous states [DAH12, PEC14, SOR16a], to study systems with single or distributed delays [SOR07, FLU10b, DAH11b, HEI11, KYR14, WIL14, LEH15b], or even to allow for discontinuous dynamical systems [LAD13, COO16]. In addition, there are several works where synchronization on networks with time evolving have been investigated [BEL04a, ZHO06f, KOH14, SOR08]. So far, however, all generalizations of the master stability approach were either introduced for a static topological structure or the interdependence between node and coupling dynamics was not taken into account.

In the case of an adaptive network the topological structure is non-constant in time and depends on the state of the network nodes. In turn, the nodal dynamics depends on the coupling structure given by the coupling adaptive weights. This subtle interplay changes the theoretical approach entirely that has to be taken in order to derive a master stability function for adaptive networks. 
In this chapter, we have developed a master stability approach and thus provided a novel extension towards adaptive networks that can be regarded as non-standard. Studying network synchronization by adapting the coupling weights [YU12, LEL10, LEH14, HOE16] is only one field of application where our new approach could be applied.

The master stability approach has been further applied to the paradigmatic model of adaptively coupled phase oscillators. We have illustrated several forms for the master stability function with respect to different adaptation rules, i.e., different values for the control parameter $\beta$. Remarkably, the emergence of bounded regions that would lead to stable synchronous dynamics has been observed in the master stability function. These bounded regions are called stability islands. We have described the structure and formation of stability islands via a cubic curve and derived an existence criterion. Additionally, we have analytically provided sufficient conditions for the system parameters which imply the stability of the synchronous state on a undirected background network structure.

In addition, the implications of the stability islands have been further explored. Using an adiabatic continuation of the synchronous state with respect to the coupling constant $\sigma$, we have obtained that the presence of a stability islands gives rise to the emergence of multicluster states and chimera-like states. The stable existence of certain multiclusters and chimera-like states have been shown numerically and analytically explained using the master stability function. Thus, the findings do not only complement the results obtained in Chapters 5 and 6 . They also underline the subtle interplay between cluster size and network structure for the stability of complex synchronization patterns. 


\section{Multilayered adaptive networks 8}

In this chapter, we show that a plethora of novel patterns can be generated by multiplexing adaptive networks. In particular, partial synchronization patterns like phase clusters and more complex cluster states which are unstable in the corresponding monoplex network can be stabilized, or even states which do not exist in the single-layer case for the parameters chosen, can be born by multiplexing. Thus our aim is to provide fundamental insight into the combined action of adaptivity and multiplex topologies. Hereby we elucidate the delicate balance of adaptation and multiplexing which is a feature of many real-world networks even beyond neuroscience [GRO06b, SHA13b, WAR14, KLI16a]. As local dynamics we use the paradigmatic Kuramoto phase oscillator model, which is a simple generic model and has been successfully applied in the modeling of synchronization phenomena in a wide range of natural and technological systems [BOC18].

A general multiplex network with $L$ layers each consisting of $N$ identical adaptively coupled phase oscillators is described by

$$
\begin{aligned}
& \dot{\phi}_{i}^{\mu}=\omega-\frac{1}{N} \sum_{j=1}^{N} \kappa_{i j}^{\mu} \sin \left(\phi_{i}^{\mu}-\phi_{j}^{\mu}+\alpha^{\mu \mu}\right)-\sum_{v=1, v \neq \mu}^{L} \sigma^{\mu v} \sin \left(\phi_{i}^{\mu}-\phi_{i}^{v}+\alpha^{\mu v}\right), \\
& \dot{\kappa}_{i j}^{\mu}=-\epsilon\left(\kappa_{i j}^{\mu}+\sin \left(\phi_{i}^{\mu}-\phi_{j}^{\mu}+\beta^{\mu}\right)\right),
\end{aligned}
$$

where $\phi_{i}^{\mu} \in[0,2 \pi)$ represents the phase of the $i$ th oscillator $(i=1, \ldots, N)$ in the $\mu$ th layer $(\mu=1, \ldots, L)$, and $\omega$ is the natural frequency. The interaction between the phase oscillators within each layer is described by the coupling matrix elements $\kappa_{i j}^{\mu} \in[-1,1]$. The intra-layer coupling weights $\kappa_{i j}^{\mu}$ are determined adaptively, whereas the inter-layer coupling weights $\sigma^{\mu \nu} \geq 0$ are fixed. The parameters $\alpha^{\mu \nu}$ are the phase lags of the interaction [SAK86]. The adaptation rate $0<\epsilon \ll 1$ is assumed to be a small parameter separating the time scales of the slow dynamics of the coupling weights and the fast dynamics of the oscillatory system. The adaptation function in each layer is chosen as in the Chapters 4 and 5.

Let us note important properties of the model. First, $\omega$ can be set to zero without loss of generality due to the shift-symmetry of Eq. (8.1), i.e., considering the co-rotating frame $\phi \rightarrow \phi+$ $\omega t$. Moreover, due to the existence of the attracting region $G \equiv\left\{\left(\phi_{i}^{\mu}, \kappa_{i j}^{\mu}\right): \phi_{i}^{\mu} \in(0,2 \pi],\left|\kappa_{i j}^{\mu}\right| \leq 1\right.$, $i, j=1, \ldots, N, \mu=1, \ldots, L\}$, one can restrict the range of the coupling weights to the interval $-1 \leq \kappa_{i j} \leq 1$ [KAS17]. Finally, based on the parameter symmetries of the model

$$
\begin{aligned}
(\boldsymbol{\alpha}, \boldsymbol{\beta}, \boldsymbol{\phi}, \boldsymbol{\kappa}) & \mapsto(-\boldsymbol{\alpha}, \pi-\boldsymbol{\beta},-\boldsymbol{\phi}, \boldsymbol{\kappa}), \\
\left(\alpha^{\mu \mu}, \beta^{\mu}, \phi_{i}^{\mu}, \kappa_{i j}^{\mu}\right) & \mapsto\left(\alpha^{\mu \mu}+\pi, \beta^{\mu}+\pi, \phi_{i}^{\mu},-\kappa_{i j}^{\mu}\right),
\end{aligned}
$$

where $\boldsymbol{\alpha}, \boldsymbol{\beta}, \boldsymbol{\phi}, \boldsymbol{\kappa}$ abbreviate the whole set of variables and parameters, it is sufficient to analyze the system within the parameter region $\alpha^{11} \in[0, \pi / 2), \alpha^{\mu \mu} \in[0, \pi)(\mu \neq 1), \alpha^{\mu \nu} \in[0,2 \pi)(\mu \neq v)$ and $\beta^{\mu} \in[-\pi, \pi)$. 
This chapter includes contents that have been published in [BER19b]. The chapter is organized as follows. In Section 8.1, we introduce the notion of lifted states and show how states from one-layer can be used to find states for multiplex systems. Additionally, the existence of new states induced by the muliplex structure is shown in Section 8.2. We describe the properties of these states and study numerically their robustness against heterogeneity ind the local dynamics. In order to study the stability of lifted states, we develop the new methodology of the multiplex decomposition in Section 8.3. Subsequently in Section 8.4, the method is applied to lifted states to prove the stabilizing features of the multiplex set-up. In Section 8.5, we provide a brief outlook on further applications of the multiplex decomposition. All findings are summarized in Section 8.6.

\subsection{Lifted states in multiplex networks}

In Chapter 4, we exhaustively described the properties of one-cluster in case of one layer and provided rigorous existence and stability results. In this section, we lift these finding to the case of two layers.

Let us now consider one-cluster states in multiplex structures. In general, one-cluster states are given as relative equilibria

$$
\begin{aligned}
& \phi_{i}^{\mu}=\Omega t+a_{i}^{\mu}, \\
& \kappa_{i j}^{\mu}=-\sin \left(a_{i}^{\mu}-a_{j}^{\mu}+\beta^{\mu}\right),
\end{aligned}
$$

with collective frequency $\Omega$ and relative phases $a_{i}^{\mu} \in[0,2 \pi)$. In order to connect one-cluster states of the single layer case to one-cluster states of the multiple layer case, we introduce the notion of lifted one-cluster states.

Definition 8.1.1 Let $\left(\phi^{1}, \ldots, \phi^{L}, \kappa^{1}, \ldots, \kappa^{L}\right)$ be a one-cluster state (8.2) solving the equations (8.1). Then $\left(\phi^{\mu}, \kappa^{\mu}\right)$ is called a lifted one-cluster state if for each layer $\mu=1, \ldots, L$ the state $\left(\phi^{\mu}, \kappa^{\mu}\right)$ is a monoplex one-cluster, i.e., $\left(\boldsymbol{\phi}^{\mu}(t), \kappa^{\mu}(t)\right)$ solves (6.1)-(6.2) and hence the distribution of phases $\boldsymbol{a}^{\mu}$ are of splay, antipodal, or double antipodal type.

In the following we show that in duplex systems $(L=2)$ the phase difference of oscillators between the layers $\Delta a \equiv a_{i}^{1}-a_{i}^{2}$ takes only two values and solves

$$
\Delta \Omega=\sigma^{12} \sin \left(\Delta a+\alpha^{12}\right)+\sigma^{21} \sin \left(\Delta a-\alpha^{12}\right),
$$

where $\Delta \Omega \equiv \Omega\left(\alpha^{11}, \beta^{1}\right)-\Omega\left(\alpha^{22}, \beta^{2}\right)$ is given in (4.6) for the three different one-cluster states (splay, antipodal, double antipodal).

To show this, suppose we have two one-cluster states where each is of either splay, antipodal, or double antipodal type which form a duplex one-cluster (8.2) for $L=2$ and $\phi_{i}^{\mu}=\Omega\left(\alpha^{\mu \mu}, \beta^{\mu}\right) t+$ $\omega^{\mu} t+a_{i}^{\mu}(\mu=1,2)$, where $\Omega\left(\alpha^{\mu \mu}, \beta^{\mu}\right)$ is given by (4.6) and the coupling weights are given by $\kappa_{i j}^{\mu}=-\sin \left(a_{i}^{\mu}-a_{j}^{\mu}+\beta^{\mu}\right)$. We verify by directly inserting that $\phi_{i}^{\mu}$ and $\kappa_{i j}^{\mu}$ solve Eq. (2.26). For the 
given ansatz, Eq. (8.1) reads

$$
\begin{aligned}
& \Omega\left(\alpha^{11}, \beta^{1}\right)+\omega^{1}=\frac{1}{2} \cos \left(\alpha^{11}-\beta^{1}\right)-\frac{1}{2} \mathfrak{R}\left(e^{-\mathrm{i}\left(2 a_{i}^{1}+\alpha^{11}+\beta^{1}\right)} Z_{2}\left(\mathbf{a}^{1}\right)\right) \\
&-\sigma^{12} \sin \left(\Delta \Omega t+\Delta \omega t+a_{i}^{1}-a_{i}^{2}+\alpha^{12}\right),
\end{aligned}
$$

and

$$
\begin{aligned}
\Omega\left(\alpha^{22}, \beta_{2}\right)+\omega^{2}=\frac{1}{2} \cos \left(\alpha^{22}-\beta^{2}\right)-\frac{1}{2} \mathfrak{R}\left(e^{-\mathrm{i}\left(2 a_{i}^{1}+\alpha^{22}+\beta^{2}\right)} Z_{2}\left(\mathbf{a}^{2}\right)\right) & \\
& +\sigma^{21} \sin \left(\Delta \Omega t+\Delta \omega t+a_{i}^{1}-a_{i}^{2}-\alpha^{21}\right) .
\end{aligned}
$$

where $\Delta \Omega=\Omega\left(\alpha^{11}, \beta^{1}\right)-\Omega\left(\alpha^{22}, \beta^{2}\right)$ and $\Delta \omega=\omega^{1}-\omega^{2}$, respectively. Thus, $\phi=\left(\phi^{1}, \phi^{2}, \kappa^{1}, \kappa^{2}\right)$ is a duplex one-cluster if

$$
\Delta \Omega+\Delta \omega=0
$$

which is equivalent to

$$
\Delta \Omega=\sigma^{12} \sin \left(a_{i}^{1}-a_{i}^{2}+\alpha^{12}\right)+\sigma^{21} \sin \left(a_{i}^{1}-a_{i}^{2}-\alpha^{21}\right)
$$

for all $i=1, \ldots, N$. Note that $\Delta \Omega$ is not necessarily zero even if the phase-lag parameters for both layers agree. They can still differ in the type of one-cluster state. The former equation can be written as

$$
\frac{\Delta \Omega}{C}=\sin \left(a_{i}^{1}-a_{i}^{2}+v\right)
$$

with

$$
\begin{aligned}
& \sin (v)=\frac{1}{C}\left(\sigma^{12} \sin \left(\alpha^{12}\right)-\sigma^{21} \sin \left(\alpha^{21}\right)\right), \\
& \cos (v)=\frac{1}{C}\left(\sigma^{12} \cos \left(\alpha^{12}\right)+\sigma^{21} \cos \left(\alpha^{21}\right)\right),
\end{aligned}
$$

where

$$
C=\sqrt{\left(\sigma^{12}\right)^{2}+\left(\sigma^{21}\right)^{2}+2 \sigma^{12} \sigma^{21} \cos \left(\alpha^{12}+\alpha^{21}\right)} .
$$

Whenever $\left(\sigma^{12}\right)^{2}+\left(\sigma^{21}\right)^{2}+2 \sigma^{12} \sigma^{21} \cos \left(\alpha^{12}+\alpha^{21}\right) \geq 0$ and

$$
\left(\sigma^{12}\right)^{2}+\left(\sigma^{21}\right)^{2}+2 \sigma^{12} \sigma^{21} \cos \left(\alpha^{12}+\alpha^{21}\right) \geq \Delta \Omega^{2},
$$

Eq. (8.4) has the two solutions $a_{i}^{1}-a_{i}^{2}=\arcsin (\Delta \Omega / C)-v$ and $a_{i}^{1}-a_{i}^{2}=\pi-\arcsin (\Delta \Omega / C)-v$. Considering the inverse function arcsin : $[-1,1] \rightarrow[-\pi / 2, \pi / 2]$ applied to Eq. (8.5) determines $v$ to be either $v^{\prime}$ or $\pi-v^{\prime}$, where $v^{\prime}:=\arcsin (\sin (v))$ and $\sin (v)$ as given in (8.5). The second equation for $\cos (v)$ then fixes $v$ to take one of the values.

The condition (8.6) is a relation between all parameters of the system which has to be fulfilled for the existence of duplex relative equilibria. Note that for any given inter-layer coupling $\sigma^{12} \neq 0$ and $\alpha^{12}+\alpha^{21} \neq \pm \pi / 2$ or $\pm 3 \pi / 2$ there exists a minimum coupling weight $\sigma^{21}<\infty$ such that the 


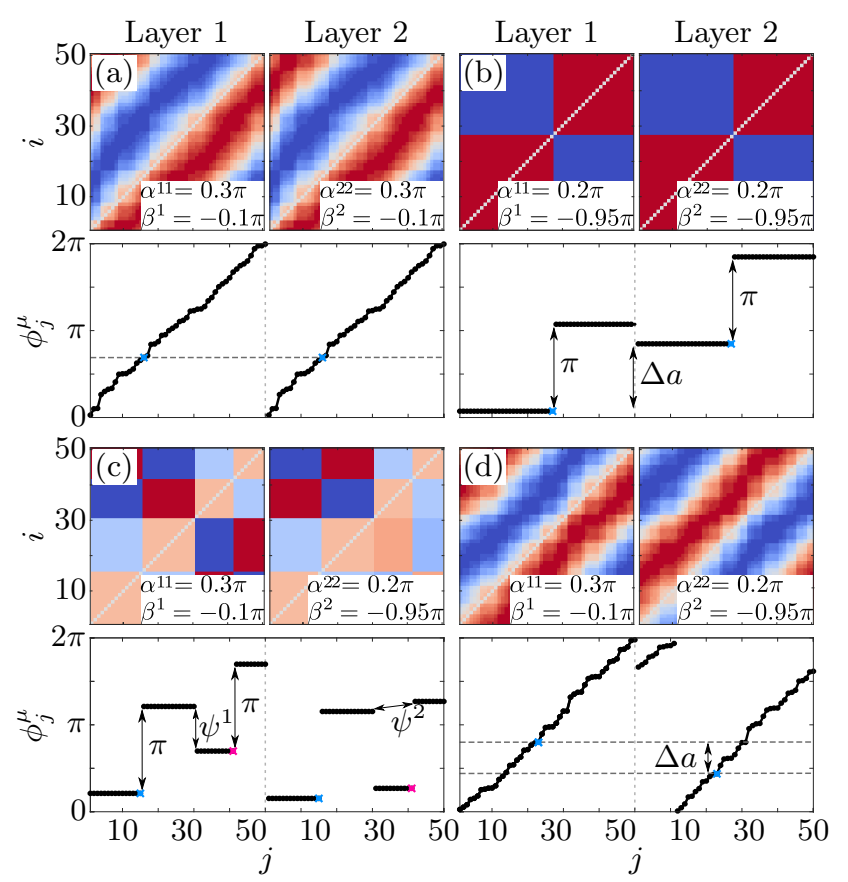

Figure 8.1: Different duplex states of Eq. (4.3) $(L=2)$ for an ensemble of 50 oscillators in each layer with color-coded coupling weights $\kappa_{i j}^{\mu}$ (upper panels, color code as in Fig.1), phases $\phi_{j}^{\mu}$ (lower panels): Duplex one-cluster states (a) of lifted splay type $\left(R_{2}\left(\mathbf{a}^{\mu}\right)=0\right)$ for $\alpha^{12 / 21}=0.3 \pi, \sigma^{12 / 21}=0.07 ;(b)$ of lifted antipodal type $\left(R_{2}\left(\mathbf{a}^{\mu}\right)=1\right)$ for $\alpha^{12}=0.3 \pi$, $\alpha^{21}=0.75 \pi, \sigma^{12 / 21}=0.62$; (c) of double antidodal type (not a lifted state) for $\alpha^{12 / 21}=0.05 \pi, \sigma^{12 / 21}=0.28$; (d) of lifted splay type for $\alpha^{12}=0.3 \pi, \alpha^{21}=0.4 \pi, \sigma^{12 / 21}=0.8$, and $\epsilon=0.01$. In the lower panels phase differences between the two layers are indicated by $\Delta a \equiv a_{i}^{1}-a_{i}^{2}$, and between the two new antipodal states (c) by $\psi^{1}, \psi^{2}$. Figure taken from [BER19b].

lifted one-clusters exist. In case of unidirectional coupling, i.e., $\sigma^{12}=0$, the condition gives the minimum weight $\sigma^{21} \geq \Delta \Omega$.

In Fig. 8.1 different duplex one-cluster states are presented that are observed by numerical simulations. Panels (a),(b),(d) in Fig. 8.1 display lifted states of splay, antipodal, and splay type, respectively. The phase distributions in both layers are the same but shifted by the constant value $\Delta a$ in agreement with the above equation. In contrast to the lifted states, Fig. 8.1(c) shows another possible one-cluster for the duplex network. Due to the interaction of the two layers we can find a phase distribution which is of double antipodal type in each layer but not a lifted state. This means that these states are born by the duplex set-up. Moreover, in contrast to the other examples the phase distribution between the layers does not agree, $\psi^{1} \neq \psi^{2}$. For the monoplex case, it has been shown that double antipodal states are unstable for any set of parameters, see Chapter 4 . Hence, finding stable double antipodal states which interact through the duplex structure is unexpected.

\subsection{Birth and robustness of phase clusters}

For more insight into the birth of phase-locked states by multiplexing, Fig. 8.2 displays the emergence of double antipodal states in a parameter regime where they do not exist in single-layer networks. They are characterized by the second moment order parameter $R_{2}$. It is remarkable that the new double antipodal state can be found for a wide range of the inter-layer coupling 


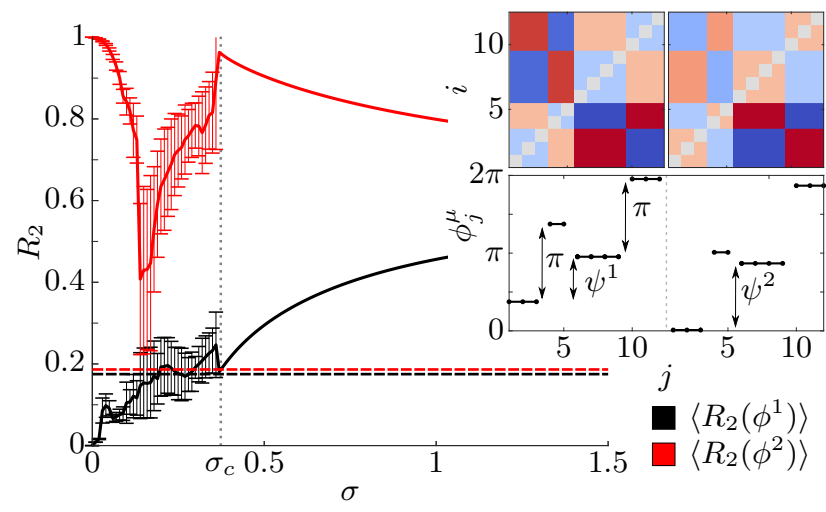

Figure 8.2: Birth of double antipodal state in a duplex network $(N=12)$ for a wide range of inter-layer coupling strength $\sigma=\sigma^{12}=\sigma^{21}$. The solid lines are the temporal averages for the second moment order parameter $R_{2}$ of the individual layers (layer 1: black, layer 2: red). The error bars for $\sigma<\sigma_{c}$ denote the standard deviation of the temporal evolution of $R_{2}$. The dashed horizontal lines represent the unique values of $R_{2}$ for the double antipodal state in a monoplex network. The plot was obtained by adiabatic continuation of a duplex double antipodal state (see inset) in both directions starting from $\sigma=0.5$. Parameters: $\alpha^{11 / 22}=0.3 \pi, \alpha^{12 / 21}=0.05, \beta^{1}=0.1 \pi, \beta^{2}=-0.95 \pi$, and $\epsilon=0.01$. Figure taken from [BER19b].

strength larger than a certain critical value $\sigma_{c}$, and is clearly different from those of the monoplex. Below the critical value $\sigma_{c}$, the double antipodal states are no longer stable, and more complex temporal dynamics occurs which causes temporal changes in $R_{2}$. This leads to non-vanishing temporal variance indicated by the error bars in Fig. 8.2.

\section{Robustness of the phase clusters for inhomogeneous natural frequencies}

In the main text, we investigate a system of identical oscillators. There the existence of particular phase cluster states of double-antipodal type is demonstrated in Figs. 8.1 and 8.2. In order to show that these states are also present in a system of heterogeneous phase oscillators, we modify the equations (8.1)-(2.26) as follows:

$$
\begin{aligned}
& \frac{d \phi_{i}^{\mu}}{d t}=\omega_{i}^{\mu}-\frac{1}{N} \sum_{j=1}^{N} \kappa_{i j}^{\mu} \sin \left(\phi_{i}^{\mu}-\phi_{j}^{\mu}+\alpha^{\mu \mu}\right)-\sum_{\nu=1, v \neq \mu}^{L} \sigma^{\mu v} \sin \left(\phi_{i}^{\mu}-\phi_{i}^{v}+\alpha^{\mu \nu}\right), \\
& \frac{d \kappa_{i j}^{\mu}}{d t}=-\epsilon\left(\kappa_{i j}^{\mu}+\sin \left(\phi_{i}^{\mu}-\phi_{j}^{v}+\beta^{\mu}\right)\right) .
\end{aligned}
$$

For the numerical analysis of system (8.7)-(8.8), we consider randomly uniformly distributed natural frequencies on the interval $[-\Delta \omega, \Delta \omega]$. To check for the robustness of the phase cluster presented in the inset of Fig. 8.2, the following steps are performed. We fix a random realization of a uniform distribution. For any inter-layer coupling strength $\sigma=\sigma^{\mu \nu}$ we take the final state from the simulation with $\omega_{i}^{\mu}=\omega_{i}^{\nu}=0$ (i.e., those obtained from Fig. 8.2) as initial condition. We perform an adiabatic continuation of the state by running the simulation for $t=5000$ and increasing the width of the distribution $\Delta \omega$ with a stepsize of 0.01 . This is done until $\Delta \omega$ reaches 0.5. The continuation is performed for each value of $\sigma$ and for 10 different realizations of the uniform distribution. Afterwards, we first check whether the final state has still the same form as the one presented in Fig. 8.2. For this, we calculate the second moment order parameter for both states in each layer individually, determine the difference of the order parameters for both layers, 


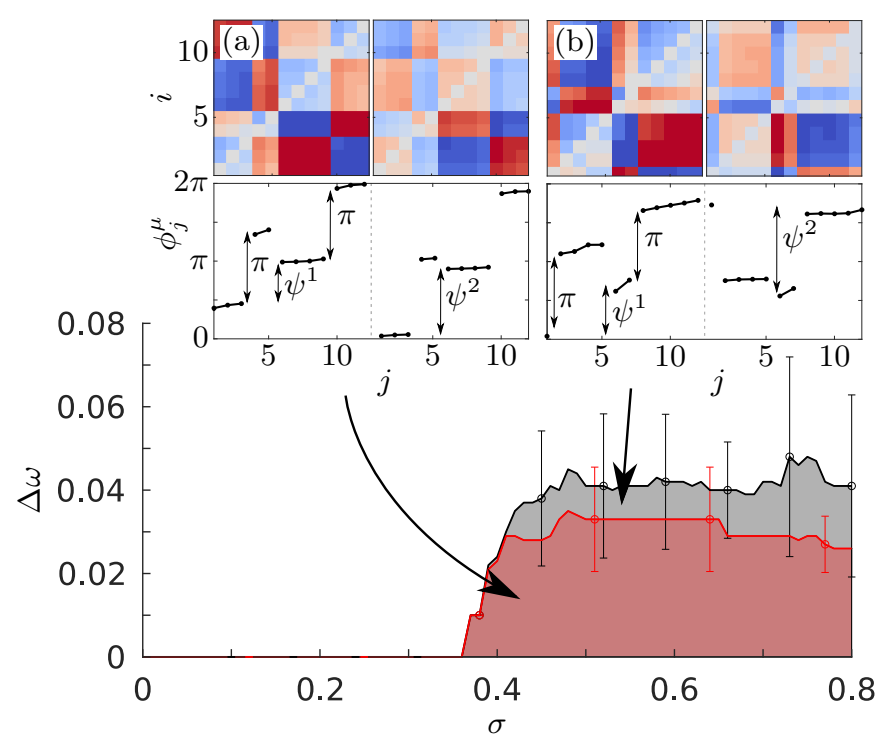

Figure 8.3: The figure shows the range $\Delta \omega$ vs where duplex one-cluster states in general (gray) and of the form presented in Fig. 3 of the main text (red) can be found. For this the system (8.7), (8.8) is integrated numerically for 10 different random uniform distributions of the natural frequencies in the interval $[-\Delta \omega, \Delta \omega]$. The results are obtained by adiabatic continuation starting with the phase clusters found for $\Delta \omega=0$ (see Fig. 3 of the main text). Duplex one-cluster states of double antipodal type with (a) $\sigma=0.5, \Delta=0.02$ and (b) $\sigma=0.5, \Delta=0.07$ are shown as insets. Parameters: $\alpha^{11 / 22}=0.3 \pi, \alpha^{12 / 21}=0.05, \beta^{1}=0.1 \pi, \beta^{2}=-0.95 \pi, \epsilon=0.01$, and $N=12$. Figure taken from [BER19b].

and set the upper limit to 0.01 . States with a difference of less than the limit are considered to possess the same form. Secondly, we check whether the final state is still a phase-locked state, i.e, all oscillators are frequency-synchronized. The range and the boundaries up to which the final state is still a duplex one-cluster state with or without the form from Fig. 8.2 are presented in Fig. 8.3. For the boundaries and the range the mean value over the 10 realizations is determined and the error bars indicate the standard deviation.

It is clearly visible that duplex one-cluster states of double-antipodal type are still present for a considerable range of heterogeneity $\Delta \omega$ of the natural frequencies. In the inset Fig. 8.3(a) we present a duplex one-cluster double-antipodal state of the same form as shown in Fig. 8.2. The phases are distorted slightly due to the frequency distribution but the double-antipodal configuration is still clearly visible. The inset Fig. 8.3(b) shows another one-cluster state of double-antipodal type. However, due to the frequency mismatch the phase distribution becomes different compared with the one presented in Fig. 8.2.

A similar result, as it is shown in Fig. 8.3, is obtained if we consider a Gaussian instead of an uniform distribution of frequencies, see Fig. 8.4. 


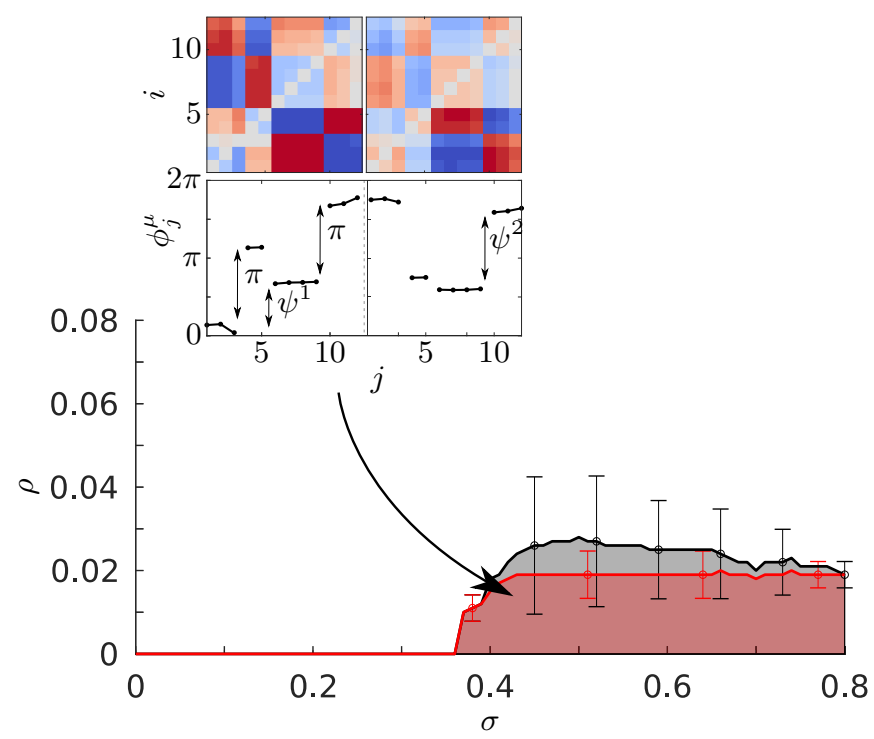

Figure 8.4: The figure shows the standard deviation $\rho$ where duplex one-cluster states in general (gray) and of the form presented in Fig. 3 of the main text (red) can be found. For this the system (8.7), (8.8) is integrated numerically for 10 different random normal distributions of the natural frequencies with standard deviation $\rho$ and zero mean. The results are obtained by adiabatic continuation starting with the phase clusters found for $\rho=0$ (see Fig. 3 of the main text). A duplex one-cluster state of double antipodal type with $\sigma=0.5, \Delta=0.02$ is shown as an inset. Parameters: $\alpha^{11 / 22}=0.3 \pi, \alpha^{12 / 21}=0.05, \beta^{1}=0.1 \pi, \beta^{2}=-0.95 \pi, \epsilon=0.01$, and $N=12$. Figure taken from [BER19b].

\subsection{Multiplex decomposition}

In this section, we provide important tools and theorems to find the spectrum of multiplex networks. These results are then subsequently used to analyze the stability of lifted one-cluster states.

Let us start with a general result on the determinant of block matrices.

Theorem 8.3.1 Let $\mathscr{R}$ be a commutative subring of $\mathbb{C}^{N \times N}$ and let $M \in \Re^{L \times L}$. Then,

$$
\operatorname{det}_{\mathbb{C}} M=\operatorname{det}_{\mathbb{C}}\left(\operatorname{det}_{\mathscr{R}} M\right) \text {. }
$$

The proof can be found in Refs. [SIL00, SOT17]. This rather abstract result allows for a very nice decomposition for pairwise commuting matrices and yields a useful tool to study the local dynamics in multiplex systems.

Proposition 8.3.2 Let $M \in \mathbb{C}^{N \times N}$ be a unitary diagonalizable matrix with $M=U D_{M} U^{H}$ where $U$, $U^{H}$ and $D_{M}$ are a unitary, its adjoint and a diagonal matrix, respectively. Let further $\mathscr{D}_{M}$ be the set of simultaneously diagonalizable matrices to $M$, i.e., the set of all matrices which commute pairwise with $M$. Then,

$$
\operatorname{det}\left(\begin{array}{ccc}
A_{11} & \cdots & A_{1 L} \\
\vdots & \ddots & \vdots \\
A_{L 1} & \cdots & A_{L L}
\end{array}\right)=\operatorname{det}\left(\sum_{\sigma \in S_{L}}\left[\operatorname{sgn}(\sigma) \prod_{\mu=1}^{L} D_{A_{\mu, \sigma(\mu)}}\right]\right)
$$


where $A_{\mu \nu} \in \mathscr{D}_{M}$ for $\mu, v=1, \ldots, L$ and $S_{L}$ is the set of all permutations of the numbers $1, \ldots, L$.

Proof. Consider any $A, B \in \mathscr{D}_{M}$, then they are simultaneously diagonalizable with $M$ and hence $A=D_{A} U^{H}$ and $B=U D_{B} U^{H}$ with the same $U$. Thus, all $A_{\mu \nu}$ can be diagonalized with the same $U$. Since $U$ is unitary,i.e. $(\operatorname{det} U)^{2}=1$, we find

$$
\operatorname{det}\left(\begin{array}{ccc}
A_{11} & \cdots & A_{1 L} \\
\vdots & \ddots & \vdots \\
A_{L 1} & \cdots & A_{L L}
\end{array}\right)=\operatorname{det}\left(\begin{array}{ccc}
D_{A_{11}} & \cdots & D_{A_{1 L}} \\
\vdots & \ddots & \vdots \\
D_{A_{L 1}} & \cdots & D_{A_{L L}}
\end{array}\right)
$$

by applying the block diagonal matrices $\operatorname{diag}(U, \cdots, U)$ and $\operatorname{diag}\left(U^{H}, \cdots, U^{H}\right)$ from the left and right, respectively. The set of diagonal matrices with usual matrix multiplication and addition form a commutative subring of $\mathbb{C}^{N \times N}$. Applying Theorem 8.3.1 and using the well-known determinant representation of Leibniz, the expression (8.9) follows.

Remark 8.3.1 The set $\mathscr{D}_{M}$ consists of all matrices which commute with $M$ and all the other elements of $\mathscr{D}_{M}$. In particular, the identity matrix $\mathbb{I}_{N} \in \mathscr{D}_{M}$ for any $M \in \mathbb{C}^{N \times N}$.

In the following, we apply the last result to a duplex and triplex system and connect the local dynamics on the one-layer network to the multiplex case. We specify our consideration by defining two special multiplex systems.

Definition 8.3.1 Suppose $A, B, C \in \mathbb{C}^{N \times N}$ and $m_{i j} \in \mathbb{C}(i, j=1, \ldots, 3)$. Then, the $2 N \times 2 N$ block matrix

$$
M^{(2)}=\left(\begin{array}{cc}
A & m_{12} \mathbb{I} \\
m_{21} \mathbb{I} & B
\end{array}\right)
$$

and the $3 N \times 3 N$ block matrix

$$
M^{(3)}=\left(\begin{array}{ccc}
A & m_{12} \mathbb{I} & m_{13} \mathbb{I} \\
m_{21} \mathbb{I} & B & m_{23} \mathbb{I} \\
m_{31} \mathbb{I} & m_{32} \mathbb{I} & C
\end{array}\right)
$$

are called (complex) duplex and triplex network, respectively.

Suppose we know how to diagonalize the individual layer topologies. The next result shows how the eigenvalues of the individual layers are connected to eigenvalues of the multiplex system. This will be done for the duplex and triplex network. For the proof of our following statement, we provide two different ways.

The first approach makes use of the Schur decomposition [BOY04, LIE15], see (4.11) in Chapter 4, which will be used, later on, in order to derive the characteristic equations. An extension of the first approach to any number of layers in the network can be found by induction but is very technical, see [KOV99, POW11a, SOT17]. The second approach uses Proposition 8.3.2 which allows for a straightforward extension to any number of layers in a multiplex network. 
Proposition 8.3.3 Suppose $A, B, C \in \mathbb{C}^{N \times N}$, they commute pairwise, and are diagonalizable with diagonal matrices $D_{A}, D_{B}, D_{C}$ and unitary matrix $U$. Then, the eigenvalues $\mu$ for the multiplex networks $M^{(2)}$ and $M^{(3)}$ can be found by solving the $N$ quadratic

$$
\mu^{2}-\left(\left(d_{A}\right)_{i}+\left(d_{B}\right)_{i}\right) \mu+\left(d_{A}\right)_{i}\left(d_{B}\right)_{i}-m_{12} m_{21}=0
$$

and cubic polynomial equations

$$
\mu^{3}+a_{2, i} \mu^{2}+a_{1, i} \mu+a_{0, i}=0
$$

respectively, with

$$
\begin{aligned}
a_{2, i} & =-\left(\left(d_{A}\right)_{i}+\left(d_{B}\right)_{i}+\left(d_{C}\right)_{i}\right) \\
a_{1, i} & =\left(d_{A}\right)_{i}\left(d_{B}\right)_{i}+\left(d_{A}\right)_{i}\left(d_{C}\right)_{i}+\left(d_{B}\right)_{i}\left(d_{D}\right)_{i} \\
& -m_{12} m_{21}-m_{13} m_{31}-m_{23} m_{32} \\
a_{0, i} & =m_{12} m_{21}\left(d_{C}\right)_{i}+m_{13} m_{31}\left(d_{B}\right)_{i}+m_{23} m_{32}\left(d_{A}\right)_{i} \\
& -\left(d_{A}\right)_{i}\left(d_{B}\right)_{i}\left(d_{C}\right)_{i}-m_{12} m_{23} m_{31}-m_{13} m_{32} m_{21}
\end{aligned}
$$

and $\left(d_{A}\right)_{i},\left(d_{B}\right)_{i}$, and $\left(d_{C}\right)_{i}$ being the respective diagonal elements of $D_{A}, D_{B}$, and $D_{C}$.

Proof. Since $A, B, C$ are diagonalizable and commute, Proposition 8.3 .2 can be applied to both matrices $M^{(2)}, M^{(3)}$. Anyhow, for the matrix $M^{(2)}$ we will provide another proof using Schur's decomposition.

The determinant is an antisymmetric multilinear form. Thus, we can write

$$
\operatorname{det}\left(M^{(2)}-\mu \mathbb{I}_{2 N}\right)=\operatorname{det}\left(\begin{array}{cc}
A-\mu \mathbb{I}_{N} & m_{12} \cdot \mathbb{I}_{N} \\
m_{21} \cdot \mathbb{I}_{N} & B-\mu \mathbb{I}_{N} m
\end{array}\right)=(-1)^{N} \operatorname{det}\left(\begin{array}{cc}
m_{12} \cdot \mathbb{I}_{N} & A-\mu \mathbb{I}_{N} \\
B-\mu \mathbb{I}_{N} & m_{21} \cdot \mathbb{I}_{N} \cdot
\end{array}\right)
$$

By assumption $A$ and $B$ are both diagonalizable with respect to the unitary transformation matrix $U$, and so are $A-\mu \mathbb{I}$ and $B-\mu \amalg$. This allows us to write

$$
\operatorname{det}\left(\begin{array}{cc}
m_{12} \cdot \mathbb{I}_{N} & A-\mu \mathbb{I}_{N} \\
B-\mu \mathbb{I}_{N} & m_{21} \cdot \mathbb{I}_{N} \cdot
\end{array}\right)=\operatorname{det}\left(\begin{array}{cc}
m_{12} \mathbb{I}_{N} & D_{A}-\mu \mathbb{I}_{N} \\
D_{B}-\mu \mathbb{I}_{N} & m_{21} \mathbb{I}_{N}
\end{array}\right)
$$

by applying the block diagonal matrices $\operatorname{diag}(U, \cdots, U)$ and $\operatorname{diag}\left(U^{H}, \cdots, U^{H}\right)$ from the left and right, respectively. Now, using Schur's decomposition (4.11) the determinant can written as

$$
\begin{aligned}
\operatorname{det}\left(\begin{array}{cc}
m_{12} \mathbb{I}_{N} & D_{A}-\mu \mathbb{I}_{N} \\
D_{B}-\mu \mathbb{I}_{N} & m_{21} \mathbb{I}_{N}
\end{array}\right) & =n^{N} \operatorname{det}\left(m-\frac{1}{n}\left(D_{A}-\mu \mathbb{I}_{N}\right)\left(D_{B}-\mu \mathbb{I}_{N}\right)\right) \\
& =\operatorname{det}\left(m_{12} m_{21} \mathbb{I}_{N}-\left(D_{A}-\mu \mathbb{I}_{N}\right)\left(D_{B}-\mu \mathbb{I}_{N}\right)\right) .
\end{aligned}
$$

The last expression together with $\operatorname{det}\left(M^{(2)}-\mu \mathbb{I}_{2 N}\right)=0$ yields the $N$ quadratic equations (8.12).

Using that $(A-\mu \mathbb{I}),(B-\mu \mathbb{I}),(C-\mu \mathbb{I})$ commute pairwise, Proposition 8.3.2 can be applied. We 
find

$$
\begin{aligned}
\operatorname{det}\left(M^{(3)}-\mu \mathbb{I}_{3 N}\right)= & \operatorname{det}\left(\left(D_{A}-\mu \mathbb{I}_{N}\right)\left[\left(D_{B}-\mu \mathbb{I}_{N}\right)\left(D_{C}-\mu \mathbb{I}_{N}\right)-m_{23} m_{32} \mathbb{I}_{N}\right]\right. \\
& \left.-m_{21}\left[m_{12}\left(D_{C}-\mu \mathbb{I}_{N}\right)-m_{13} m_{32} \mathbb{I}_{N}\right]+m_{31}\left[m_{12} m_{23} \mathbb{I}_{N}-m_{13}\left(D_{B}-\mu \mathbb{I}_{N}\right)\right]\right)
\end{aligned}
$$

The last expression together with $\operatorname{det}\left(M^{(3)}-\mu \mathbb{I}_{3 N}\right)=0$ yields the $N$ cubic equations (8.13).

Let us briefly discuss some special cases for both the duplex and triplex network. Consider a duplex network with master and slave layer, i.e., either $m_{12}=0$ or $m_{21}=0$. Then, the quadratic equations (8.12) yield

$$
\left(\mu-\left(d_{A}\right)_{i}\right)\left(\mu-\left(d_{B}\right)_{i}\right)=0
$$

As shown in Proposition 8.3.2, the eigenvalues for special triplex networks can be found by solving cubic equations. For the solution even closed forms exist. Despite this, the explicit form of the solutions is rather tedious, in general. However, if we consider $A=B=C$ and a ring-like inter-layer connection between the networks, i.e., $m_{12}=m_{23}=m_{31}=0$, then equation (8.13) has the following solutions for all $j=1, \ldots, N$

$$
\begin{aligned}
& \mu_{1}=-\left(d_{A}\right)_{j}+\left(m_{13} m_{32} m_{21}\right)^{1 / 3}, \\
& \mu_{2}=-\left(d_{A}\right)_{j}+\frac{1}{2} \mathrm{i}(\mathrm{i}+\sqrt{3})\left(m_{13} m_{32} m_{21}\right)^{1 / 3}, \\
& \mu_{3}=-\left(d_{A}\right)_{j}-\frac{1}{2}(\mathrm{i}+\sqrt{3})\left(m_{13} m_{32} m_{21}\right)^{1 / 3},
\end{aligned}
$$

where i denotes the imaginary unit. In analogy to equation (8.14), a decoupling for the eigenvalues can be found. Consider three pairwise commuting matrices $A, B, C$, and the structure between the layers is a directed chain, i.e., $m_{12}=m_{13}=m_{31}=m_{23}=0$, then

$$
\left(\mu-\left(d_{A}\right)_{i}\right)\left(\mu-\left(d_{B}\right)_{i}\right)\left(\mu-\left(d_{C}\right)_{i}\right)=0 .
$$

In the following we apply the results of this section to the stability analysis of lifted duplex one-clsuter states. In the last Section 8.5 of this chapter, further two different applications of the multiplex decomposition are briefly discussed.

\subsection{Stabilizing through multiplexing}

In the following we show how the dynamics in a neighborhood of a single layer one-cluster state can be lifted to the dynamics in a neighborhood of lifted multiplex one-cluster state, i.e., we investigate and relate their local stability features. Everything is exemplified for antipodal type states but can be generalized to lifted splay and lifted double antipodal states in a straightforward manner. To study the dynamics around the one-cluster states described by Eq. (8.2), we linearize 
Eq. (8.1) around these states:

$$
\begin{aligned}
\dot{\delta \phi_{i}^{\mu}=} & \frac{1}{N} \sum_{j=1}^{N}\left[\sin \left(\Delta a+\beta^{\mu}\right) \cos \left(\Delta a+\alpha^{\mu \mu}\right) \Delta_{i j}^{\mu \mu} \delta \phi\right. \\
& \left.-\sin \left(\Delta a+\alpha^{\mu \mu}\right) \delta \kappa_{i j}^{\mu}\right]-\sum_{v=1}^{M} \sigma^{\mu \nu} \cos \left(\Delta a+\alpha^{\mu \nu}\right) \Delta_{i j}^{\mu v} \delta \phi, \\
\dot{\delta \kappa_{i j}^{\mu}=} & -\epsilon\left(\delta \kappa_{i j}^{\mu}+\cos \left(\Delta a+\beta^{\mu}\right) \Delta_{i j}^{\mu \mu} \delta \phi\right)
\end{aligned}
$$

where $\Delta_{i j}^{\mu v} \delta \phi \equiv \delta \phi_{i}^{\mu}-\delta \phi_{j}^{\nu}$.

Consider a duplex antipodal one-cluster state Eq. (8.2) with $a_{i}^{1} \in\{0, \pi\}$ and $a_{i}^{2}=a_{i}^{1}-\Delta a$, Eq. (8.16) can be brought to the duplex form in Definition (8.3.1) and possesses the following set of eigenvalues

$$
\oint_{\text {Duplex }}=\left\{-\epsilon,\left(\lambda_{i, 1}, \lambda_{i, 2}, \lambda_{i, 3}, \lambda_{i, 4}\right)_{i=1, \ldots, N}\right\}
$$

where $\lambda_{i, 1, \ldots, 4}$ solve the following $N$ quartic equations

$$
(\lambda+\epsilon)^{2} m_{1} m_{2}-\left[\left(\lambda-\rho_{i, 1}^{1}\right) \cdot\left(\lambda-\rho_{i, 2}^{1}\right)+m_{1}(\lambda+\epsilon)\right]\left[\left(\lambda-\rho_{i, 1}^{2}\right)\left(\lambda-\rho_{i, 2}^{2}\right)+m_{2}(\lambda+\epsilon)\right]=0,
$$

with $m_{1}=\sigma^{12} \cos \left(\Delta a+\alpha^{12}\right), m_{2}=\sigma^{21} \cos \left(\Delta a-\alpha^{21}\right)$ and the eigenvalues $\rho_{i, 1,2}^{\mu} \equiv \rho_{i, 1,2}\left(\alpha^{\mu \mu}, \beta^{\mu}\right)$ for the monoplex system, see Corollary 4.2.3. Using the real parts of the eigenvalues of this analysis for the duplex antipodal clusters, the stability for duplex antipodal states is found. All details and the proof of (8.17) is provided in the appendix A.7.

We have seen that the stability analysis of the duplex system can be reduced to that of the monoplex case. Thus, we are now able to analyze the stabilizing and destabilizing features of a duplex network numerically and analytically. To illustrate the effect of multiplexing, the interaction between two clusters of antipodal type is presented in Fig. 8.5. The stability of these states is determined by integrating Eq. (4.3) numerically starting with a slightly perturbed lifted antipodal state. The states are stable if the numerical trajectory is approaching the lifted antipodal state. Otherwise, the state is considered as unstable. The black contour lines in Fig. 8.5 show the borders of the stability regions in dependence of the coupling strength $\sigma^{21}$, as calculated from the Lyapunov exponents.The borders are in remarkable agreement with the numerical results.

We investigate two different situations in Fig. 8.5: In both panels the parameters for the first layer $\alpha^{11}, \beta^{1}$ are chosen such that the antipodal state is stable without inter-layer coupling. The stability of the duplex antipodal states is displayed in the $\left(\alpha^{22}, \beta^{2}\right)$ parameter plane for several values of the inter-layer coupling $\sigma^{21}$. To compare the effects of the duplex network with the mono-layer case, the stability regions for monoplex antipodals states are displayed, as well, as red hatched areas. They are markedly different. In Figure 8.5(a), the two layers are connected unidirectionally $\left(\sigma^{12}=0\right)$. It can be seen that with increasing inter-layer coupling weight $\sigma^{21}$ the region of stability for the lifted antipodal state also grows. Already for small values of the inter-layer couplings $\sigma^{21}$, a stabilizing effect of the duplex network can be noticed. For $\sigma=0.1$ there exist already regions for which the duplex antipodal state is stable but the corresponding monoplex state would not be stable. The opposite effect is found as well where the duplex network destabilizes a lifted state. Figure 8.5(b) shows the results for two layers with bidirectional coupling. In this 


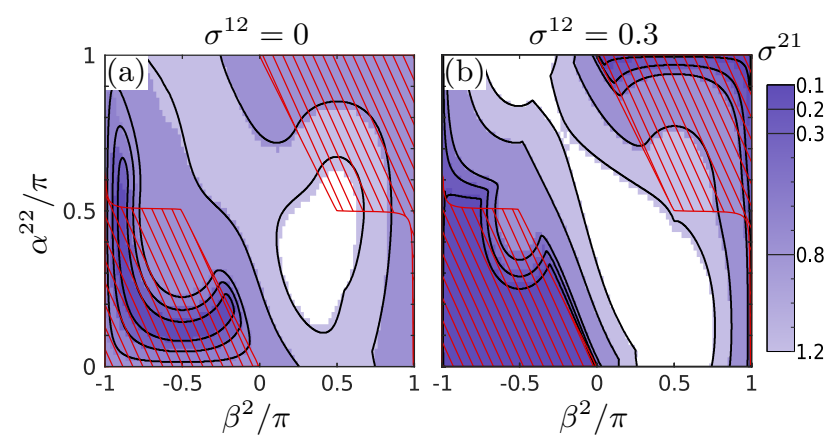

Figure 8.5: Regions of stability (blue) and instability (white) of the lifted antipodal state in the $\left(\alpha^{22}, \beta^{2}\right)$ parameter plane for different values of interlayer coupling (indicated by different blue shading) $\sigma^{21}$, where regions of stronger coupling $\sigma^{21}$ (lighter blue) include such of weaker $\sigma^{21}$ (darker blue). Stability regions for single-layer antipodal clusters are indicated by red hatched areas. The inter-layer coupling is considered as (a) unidirectional $\left(\sigma^{12}=0\right)$ and (b) bidirectional $\left(\sigma^{12}=\sigma^{21}\right)$. Parameters: $\alpha^{11}=0.2 \pi, \beta^{1}=-0.8 \pi, \alpha^{12}=0, \alpha^{21}=0.3 \pi$, and $\epsilon=0.01$. Figure taken from [BER19b].

case, the duplex structure can have stabilizing and destabilizing effects, as well. Further, for the bidirectional coupling we also notice a growth of the stability region with increasing $\sigma^{21}$ similar to the unidirectional case. However, the regions of stability grow at different rates in dependence on $\sigma^{21}$ and non-monotonically with respect to the parameters $\alpha^{22}, \beta^{2}$. Comparing the size of the stability region for both cases, one can see that for small values of $\sigma^{21}$ the region for bidirectional coupling is larger. In turn, for higher inter-layer coupling, the regions for the unidirectional case are larger. It is worth noting that in Fig. 8.5 the stability regions for smaller values of $\sigma^{21}$ are always contained in the region for larger values of $\sigma^{21}$.

\subsection{Applications for the multiplex decomposition}

In the previous section, we have shown how the multiplex decomposition can be applied to understand the stability features of lifted states. Here, we provide two additional perspective where the newly derived decomposition can be used to generalize existing results and make others analytically accessible.

\subsubsection{The master stability approach for multiplex networks}

In Ref. [TAN19], the master stability function for dynamical systems on multiplex networks was introduced. In their article, the authors considered diffusive systems similar to (2.10). Consider now the synchronous solution which solves $\dot{\mathbf{s}}=f(\mathbf{s})$. The master stability function is then derived from the following variational equations

$$
\dot{\xi}=\left[\mathbb{I}_{N L} \otimes D f(\mathbf{s})-\sigma\left(\mathscr{L}^{\text {intra }} \otimes H\right)-\rho\left(\mathscr{L}^{\text {inter }} \otimes G\right)\right] \xi
$$

where $H$ and $G$ are linear coupling functions. Here, $\xi=\mathbf{x}-\mathbb{I}_{L} \otimes \mathbb{I}_{N} \otimes \mathbf{s}$ and $\mathbf{x} \in \mathbb{C}^{L \cdot N \cdot d}$ is the system state vector where all individual nodal states are stacked on each other ordered by the 
layer and node index. Further, the intra-layer Laplacian is defined is defined as

$$
\mathscr{L}^{\text {intra }}=\bigoplus_{l=1}^{L} L^{l}=\left(\begin{array}{lll}
L^{1} & & \\
& \ddots & \\
& & L^{L}
\end{array}\right)
$$

where

$$
L^{k}=\left(\begin{array}{ccc}
\sum_{j=1}^{N} a_{1 j}^{k} & & \\
& \ddots & \\
& & \sum_{j=1}^{N} a_{N j}^{k}
\end{array}\right)-A^{k} .
$$

The inter-layer Laplacian is defined as $\mathscr{L}^{\text {inter }}=L^{I} \otimes \mathbb{I}_{N}$ where

$$
L^{I}=\left(\begin{array}{lll}
\sum_{l=1}^{L} m^{1 l} & & \\
& \ddots & \\
& & \sum_{l=1}^{L} m^{L l}
\end{array}\right)-M,
$$

where $m^{k l}$ with $m^{l l}=0$ are the entries of the $L \times L$ matrix $M$. All further necessary details on the model equations can be found in Ref. [TAN19].

In Ref [TAN19], it was shown that if $\mathscr{L}^{\text {intra }}$ and $\mathscr{L}^{\text {inter }}$ commute, a master stability equation for system (8.18) can be found which reads

$$
\dot{\mathbf{y}}=[D f(\mathbf{s})-\alpha H-\beta G] \mathbf{y},
$$

where $\alpha=\sigma \lambda, \beta=\rho \mu, \lambda$ and $\mu$ are the (complex) eigenvalues of $\mathscr{L}^{\text {intra }}$ and $\mathscr{L}^{\text {inter }}$, respectively. Note that if $H=G$ the master stability equation can be reduced to

$$
\dot{\mathbf{y}}=[D f(\mathbf{s})-\gamma H] \mathbf{y},
$$

with $\gamma=\alpha+\beta$ and $\mathbf{y} \in \mathbb{C}^{d}$. Equation (8.19) is called the master stability equation for the composite system where a single supra-Laplacian matrix $\sigma \mathscr{L}^{\text {intra }}+\rho \mathscr{L}^{\text {inter }}$ described the network topology [DOM13, KIV14].

Direct evaluation shows that $\left[\mathscr{L}^{\text {intra }}, \mathscr{L}^{\text {inter }}\right]=0$ is equivalent to $m^{k l} L^{l}-m^{l k} L^{k}=0$ for all $l, k=1, \ldots, L$. Thus, to require $\left[\mathscr{L}^{\text {intra }}, \mathscr{L}^{\text {inter }}\right]=0$ yields a linear dependence between the individual layer topologies. Using Proposition 8.3.1 for composite system, the master stability function can be used under much milder conditions.

Proposition 8.5.1 Let us consider the multiplex dynamical system (8.18), with linear function $H=G$. Suppose that further all $L^{l}, l=1, \ldots, L$, commute pairwise. Then, the master stability equation is given by

$$
\dot{\mathbf{y}}=[D f(\mathbf{s})-\mu H] \mathbf{y},
$$

and $\mu=\mu\left(\lambda_{i}^{1}, \ldots, \lambda_{i}^{l}\right)$ being a non-linear function, mapping the ith eigenvalues $\lambda_{i}^{l}(i=1, \ldots, N)$ of the layer topologies $L^{l}$, to the master function parameter $\mu$ in (8.20). The non linear mapping is given as the 
formal solution to the Lth order polynomial equation

$$
\operatorname{det}\left(\sum_{\sigma \in S_{L}}\left[\operatorname{sgn}(\sigma) \prod_{l=1}^{L} D_{\left(L_{l \sigma(l)}^{\text {supra }}-\mu \mathbb{I}_{N} \delta_{l \sigma(l)}\right)}\right]\right)=0,
$$

where $\mathscr{L}^{\text {supra }}=\sigma \mathscr{L}^{\text {intra }}+\rho \mathscr{L}^{\text {inter }}$ is a $L \times L$ block matrix divided into $N \times N$ matrices which we individually refer to with $\mathscr{L}_{k l}^{\text {supra }}, k, l=1, \ldots, L$, and $\delta_{k l}$ is the Kronecker symbol.

Proof. Using $H=G$, the variation equation for (8.18) on the synchronous solution $\mathbf{s}(t)$ is given by

$$
\dot{\boldsymbol{\xi}}=\left[\mathbb{I}_{N L} \otimes D f(\mathbf{s})-\mathscr{L}^{\text {supra }} \otimes H\right] \xi
$$

By assumption all $L^{l}, l=1, \ldots, L$, commute pairwise and $\mathscr{L}^{\text {inter }}$ is a $L \times L$ block matrix consisting of $N \times N$ identity matrices multiplied by scalar. Hence, Proposition 8.3 .2 can be applied to $\left(\mathscr{L}^{\text {supra }}-\mu \mathbb{I}_{L \cdot N}\right)$ in order to diagonalize $\mathscr{L}^{\text {supra }}$.

With this Proposition, we have a very powerful tool in order to investigate not only the influence of the multiplex structure network on the stability of the synchronous state but also the impact of different layer topologies which are not necessarily linearly dependent. As an example, we consider a duplex systems with $\left[L^{1}, L^{2}\right]=0$ and

$$
\mathscr{L}^{\text {supra }}=\left(\begin{array}{cc}
\sigma L^{1}+\rho m^{12} \mathbb{I} & -\rho m^{12} \mathbb{I} \\
-\rho m^{21} \mathbb{I} & \sigma L^{2}+\rho m^{21} \mathbb{I}
\end{array}\right) .
$$

Knowing the eigenvalues for $L^{1}$ and $L^{2}$, the master function parameter $\mu$ is determined using Eq. (8.12). The matrices $L^{1}$ and $L^{2}$ are Laplacian matrices which have at least one zero eigenvalue, corresponding to the so-called Goldstone mode $\hat{1}=(1, \ldots N \text {-times } \cdots, 1)^{T}$. As a result there are two parameters $\mu=0$ and $\mu=\rho\left(m^{12}+m^{21}\right)$. The first value corresponds to the Goldstone mode $(\hat{1}, \hat{1})^{T}$. The second parameter is exclusively induced by the duplex structure and completely independent from the individual layer topologies. Further, we find that in case of $m^{12}=-m^{21}$ another zero parameter $\mu$ exists which corresponds to the eigenmode $(\hat{1},-\hat{1})^{T}$. The other eigenvalues of the supra-Laplacian matrix $\mathscr{L}^{\text {supra }}$ are given by the non-linear mappings

$$
\mu\left(\lambda^{1}, \lambda^{2}\right)=\frac{\sigma\left(\lambda^{1}+\lambda^{2}\right)+\rho\left(m^{12}+m^{21}\right)}{2} \pm \frac{1}{2} \sqrt{\left(\sigma\left(\lambda^{1}-\lambda^{2}\right)+\rho\left(m^{12}-m^{21}\right)\right)^{2}+4 \rho^{2} m^{12} m^{21}}
$$

for which we have formally solved equation (8.12) with respect to $\mu$. Let us consider two special cases.

First, we assume that there is no connection from the second to the first layer. We have a masterslave set-up which means that $m^{12}=0$. With this, the master function parameter is $\mu\left(\lambda^{1}, \lambda^{2}\right)=\sigma \lambda^{1}$ and $\mu\left(\lambda^{1}, \lambda^{2}\right)=\sigma \lambda^{2}+\rho m^{21}$. Remarkably, in this set-up the stability of a synchronous state in a duplex network is reduced to the pure one-layer system. The stability in the duplex system is determined by the spectrum of the individual layer topologies where only in the second layer the spectrum is shifted due to the interaction. 
The second case starts from the consideration in [TAN19]. In particular, we consider [ $\left.\mathscr{L}^{\text {intra }}, \mathscr{L}^{\text {inter }}\right]=$ 0 which leads to a pairwise linear dependence of all individual layer topologies, and hence $\lambda_{i}^{l}=\lambda_{i}$ for all $l=1, \ldots, L$ and $i=1, \ldots, N$ with $\lambda_{i}^{l} \in \mathbb{C}$. Taking this into account, the equation for the master function parameter yields $\mu\left(\lambda^{1}, \lambda^{2}\right)=\sigma \lambda^{1}+\rho\left(m^{12}+m^{21}\right)$ and $\mu\left(\lambda^{1}, \lambda^{2}\right)=\sigma \lambda^{1}$. In order to see that the master function parameter agrees with the set for the parameter $\gamma$ in (8.19), we determine the eigenvalues of $\mathscr{L}^{\text {intra }}$ and $\mathscr{L}^{\text {inter }}$ individually. Since $L^{(1)}$ and $L^{(2)}$ are linearly dependent, the set of eigenvalues for $\mathscr{L}^{\text {intra }}$ consists of the eigenvalues of $L^{1}$ with double multiplicity. Using Proposition 8.3.3, we find that $\mathscr{L}^{\text {inter }}$ has eigenvalues 0 and $\left(m^{12}+m^{21}\right)$ each with multiplicity $N$. Since both Laplacian matrices commute, there exists a common set of eigenvectors. We find $\gamma=\rho \lambda^{(1)}$ for the eigenvector $(1,1)^{T} \otimes v$ and $\gamma=\rho \lambda^{(1)}+\sigma\left(m_{12}+m_{21}\right)$ for the eigenvector $\left(m_{12},-m_{21}\right)^{T} \otimes v$ where $L^{1} v=\lambda^{1} v$. Here, again everything boils down to a pure one-layer set up with an additional shift.

\subsubsection{Analytic treatment of diffusive dynamics on multiplex networks}

In the previous section we considered the dynamics of linear systems as they are given by the variational equation (8.18). In the context of diffusive systems on complex networks, recently linear diffusive processes were considered in order to study the dynamics on social as well as transport networks [BAR11d, GOM13]. Compared with equation in [GOM13], the authors investigate a duplex system $(L=2)$ with $f=0, \sigma=D_{k}(k=1,2), H, G=\mathbf{I}$, and $\rho m^{k l}=D_{x}$. Additionally, they considere weighted networks for which instead of $a_{i j}^{k} \in\{0,1\}$ coupling weights $\kappa_{i j}^{k}(k=1,2)$ were taken. Note that the former results on multiplex matrices still hold true for weighted connections.

Let us assume that the super-Laplacian matrix is given by

$$
\mathscr{L}^{\text {supra }}=\left(\begin{array}{cc}
D_{1} L^{1}+D_{x} I & -D_{x} \mathbb{I} \\
-D_{x} \mathbb{I} & D_{2} L^{2}+D_{x} I
\end{array}\right) .
$$

with $\left[L^{1}, L^{2}\right]=0$. Due to the structure of the supra-Laplacian we are allowed to apply Proposition 8.5.1. In accordance with [GOM13] and our former findings in Section 8.5.1, we have the two eigenvalues $\mu=0$ and $\mu=2 D_{x}$ corresponding to the Goldstone mode $(\hat{1}, \hat{1})^{T}$ and the vector $(\hat{1},-\hat{1})^{T}$, respectively. The other eigenvalues are given as solution to the equations (8.12) and read

$$
\mu_{i}=\frac{D_{1} \lambda_{i}^{1}+D_{2} \lambda_{i}^{2}+2 D_{x}}{2} \pm \frac{1}{2} \sqrt{\left(D_{1} \lambda_{i}^{1}-D_{2} \lambda_{i}^{2}\right)^{2}+4\left(D_{x}\right)^{2}}
$$

where $\lambda_{i}^{1}$ and $\lambda_{i}^{2}$ are the eigenvalues of the Laplacian matrices $L^{1}$ and $L^{2}$, respectively. With this we derived a complete analytic expression for the spectral properties of the diffusive system which was considered in [GOM13] without using any perturbation method. 


\subsection{Summary}

In summary, we have proposed a concept to induce diverse partial synchronization patterns (phase clusters) in adaptively coupled phase oscillator networks. While adaptive networks have recently attracted a lot of attention in the fields of neuroscience and social sciences, biology, engineering, and other disciplines, and multilayer networks are a paradigm for real-world complex networks, little has been known about the interplay of multilayer structures and adaptivity. We have aimed to fill this gap within a rigorous framework of theoretical analysis and computer simulations. We have shown that multiplexing in a multi-layer with symmetry can induce various stable phase cluster states like splay states, antipodal states, and double antipodal states, in a situation where they are not stable or do not even exist in the single layer.

Further, we have developed a novel method for analysis of Laplacian matrices of duplex networks which allows for insight into the spectral structure of these networks, and can easily be generalized to more than two layers. This new approach of multiplex decomposition has a broad range of applications to physical, biological, socio-economic, and technological systems, ranging from plasticity in neurodynamics or the dynamics of linear diffusive systems [GOM13, SOL13a] to generalizations of the master stability approach [PEC98, TAN19] for adaptive networks. We have used the multiplex decomposition to provide analytic results for the stability of lifted states in the multilayer system. As local dynamics we have used the paradigmatic Kuramoto phase oscillator model, supplemented by adaptivity of the link strengths with a phase lag parameter which can model a whole range of adaptivity rules from Hebbian via spike-timing dependent plasticity to anti-Hebbian. 


\section{Conclusion and Outlook 9}

Part I of this thesis was devoted to the analysis of cluster states in populations of globally coupled neurons with synaptic plasticity. Here, we used mathematical as well as numerical tools in order to understand the mechanism behind the emergence of frequency clustering.

In Chapter 3, we showed that adaptive neural networks are able to generate self-consistently dynamics with different frequency bands. Here, each cluster corresponds to a strongly connected component with a fixed frequency. Due to a sufficiently large difference of the cluster sizes and frequencies, the inter-cluster interactions are suppressed, while the intra-cluster interactions are enhanced. In this study, we described the mechanisms behind the formation and stabilization of these clusters. In particular, we explained why the significant difference between the cluster sizes is important for the decoupling of the clusters. From a broader perspective, the decoupling of the clusters in our case is analogous to the decoupling of timescales in systems with multiple timescales.

In addition, we presented a two-dimensional phenomenological model which allows for a detailed study of the clustering mechanisms. Despite of the approximations made by the derivation, the model coincides surprisingly well with the adaptive Hodgkin-Huxley network. Using the phenomenological model, we found parameter regions of the plasticity function, where stable frequency clustering can be observed.

Clustering behavior also emerges at the brain scale, where synchronized communities of brain regions constituting large distributed functional networks can intermittently be formed and dissolved [DEC09, PON15]. Such clustering dynamics can shape the structured spontaneous brain activity at rest as measured by fMRI. In this study, we showed that slow oscillations based on the modulation of synchronized neural activity can already be formed at the resolution level of a single neural population if adaptive synapses are taken into account. These modulations of the amplitude of the mean field can be generated in a stable manner, see Fig.3.9 and Ref. [POP15]. The mechanism relies on fluctuations of the extent of synchronization of tonically firing neurons that are neurons which fire periodically provided they receive a constant synaptic input. The fluctuations are caused by the splitting of the neural population into clusters and the corresponding cluster dynamics. Our findings might contribute to the emergence of slow brain rhythms of electrical (LFP, EEG) and metabolic (BOLD) brain activity reported by [MAN07, MAG12a, MON08, ALV14].

However, other mechanisms for generating slow oscillations are possible. The papers [BAZ02, COM03] discussed the emergence of slow oscillatory activity $(<1 \mathrm{~Hz})$ that can be observed in vivo in the cortex during slow-wave sleep, under anesthesia or in vitro in neural populations. The suggested mechanism relies on the corresponding modulation of the firing of individual neurons, and the slow oscillation at the population level was proposed to be the result of very slow bursting of individual neurons that synchronize across the neural population. In contrast, the presented work shows that the slow oscillations of the population mean field can also emerge when the firing of individual neurons is not affected. The neurons may tonically fire 
at high frequencies. The amplitude of the population mean field then oscillates at much lower frequencies due to the slow modulation caused by the cluster dynamics.

Additionally, we would like to mention that the observed frequency clustering resembles phenomenologically the weak chimera states [ASH15, BIC16b] where clusters with different frequencies are formed in symmetrically coupled oscillators without adaptation. However the properties and mechanisms of the appearance of such clusters are different from those presented here, which are essentially based on the slow adaptation.

In Chapter 3.4 the phenomenological phase oscillator model was shown to be a very powerful tool to predict the clustering dynamics of neuronal population with synaptic plasticity. It is wellknown that various models of weakly coupled oscillatory systems can be reduced to coupled phase oscillators. For this, we implemented a simplified phase oscillator model in Chapters 4-5 which is able to describe the slow adaptive change of the network depending on the oscillatory states. The slow adaptation is controlled by a time-scale separation parameter.

In this framework, we developed the analytical approach of building blocks for multicluster states. We showed that for the case of phase oscillators the multiclusters are composed of certain one-cluster states (building blocks) that are themselves phase-locked solutions of the adaptive model. Moreover, we provided rigorous conditions under which multiclusters emerge and can be found stable. For the analysis we divided the problem into two main step. Firstly, we analyzed the single one-cluster states. Secondly, the multicluster states were analytically described and were shown to inherit important properties from their building blocks.

In Chapter 4, we exhaustively described the one-cluster states in an adaptive network of globally coupled phase oscillators. In particular, the following three types of one-cluster (relative equilibria) are possible building blocks for multicluster states: splay, antipodal, and double antipodal. In order to understand the stability of these states, we performed a linear stability analysis for the relative equilibria. The stability of these states was rigorously described, and the impact of all parameters was shown. We showed that there exists an $N-2$ dimensional family of splay type cluster states which gives rise to $N-2$ neutrally stable directions. Remarkably, this property of splay states was also found in networks of pulse-coupled rotators [CAL09a] and excitable neurons [DIP12]. We further proved that the double antipodal states are unstable in the whole parameter range. They appear to be saddle-points in the phase space. While the time-scale separation has no influence upon their existence, it plays an important role for the stability the relative equilibria. The regions of stability in parameter space were presented for different choices of the time-scale separation parameter. The singular limit $(\epsilon \rightarrow 0)$ and the limit of instantaneous adaptation were analyzed. The latter shows that the stability region of the splay and the antipodal states divide the whole space into two equally sized regions without intersection. Instantaneous adaptation cancels multistability of these states. The consideration of the singular limit showed that it differs from the case of no adaptation. Therefore, even for very slow adaptation, the oscillatory dynamics alone is not sufficient to describe the stability of the system.

Subsequently, the role of double antipodal states was discussed. We found that in a system of 3 oscillators these states are transient states in a small heteroclinic network between antipodal and splay states. They appear to be metastable, i.e. observable for a relative long time and therefore are physically important transient states. Moreover an additional analysis for an ensemble of 100 
phase oscillators revealed the importance of the double antipodal states for the global dynamics of the whole system.

In Chapter 5, we described the appearance of several different frequency cluster states. Starting from random initial conditions, our numerical simulations showed two different types of states. These are the splay and the antipodal type multicluster states. A third mixed type multicluster state was found by using well prepared initial condition. For all these states the collective motion of oscillators, the shape of the network, and the interaction between the frequency clusters was presented in detail. It turned out that the oscillators are able to form groups of strongly connected units. The interaction between the groups is weak compared to the interaction within the groups.

While the one-cluster are relative equilibria of the model due to the phase-shift symmetry, the multicluster states contain components with different frequencies, and, hence, they cannot be reduced to an equilibrium by transforming into another co-rotating frame. As a result, the study of multiclusters is more involved. However, to our surprise, we were still able to find an explicit form of multiclusters with the components of the splay type. Remarkably, in addition to its ring-like spatial structure that dynamically emerges, the network behaves in such a case (quasi-)periodically in time such that the whole solution can be interpreted as a spatio-temporal wave. Moreover, while the existence of the one-cluster states does not depend on the time-scale separation parameter, the multicluster states crucially depend on the timescale separation. In fact, we provided an analysis showing that there exists a critical value for the time-scale separation. Moreover, we showed that in the case of two-cluster states of splay type the adaptation of the coupling weights must be at most half as fast as the dynamics of the oscillators. For the splay clusters we analytically provided an explicit existence criterion. This fact is of crucial importance for comparing dynamical scenarios induced by short-term or long-term plasticity [FRO16].

In contrast to the splay type multicluster, the analysis of multicluster states of antipodal type is more subtle due to the modulation of the frequency. More specifically, we looked at multiclusters with bounded frequency modulation. For these types of multiclusters, we derived an asymptotic expansion in the parameter $\epsilon$ that gave explicit existence conditions. In addition, we showed analytically the existence of mixed multiclusters, which consist of clusters of splay type and clusters of antipodal type. For the mixed multiclusters, the temporal behavior within one cluster was shown to be slightly non-identical, namely, the oscillators possess the same averaged frequency, but they still can have a bounded quasi-periodically modulated phase difference.

The stability of two-cluster states was analyzed numerically and was presented for different values of the time-scale separation parameter. By assuming weakly interacting clusters, we described the stability of the two-cluster with the help of the analysis of one-cluster states. The simulations showed that there are no stable two-cluster states with clusters of the same size. We provided an argument to understand this property of the system.

Moreover, the findings on multicluster solutions as they are reported in this work are in very good agreement with previous results on adaptive neural networks [POP15, CHA17a]. Here, stable multicluster states of coherently spiking neurons with weak but time-dependent inter-cluster coupling were reported. With this work we shed some light on these generic time-dependent network patterns. 
In Part II, we extended the findings of the first part towards more complex connectivity structures.

In Chapter 6, a model of adaptively coupled identical phase oscillators on a nonlocal ring was studied. Various frequency synchronized states were observed including one-cluster, multicluster, and solitary states. Those states are similar to those found for a global base topology [KAS17, KAS18a]. However, to account for the complex base topology, we introduced a new definition of one-cluster states by means of connected induced subnetworks. This definition allows furthermore to distinguish between multicluster and solitary states in a more rigorous way than it had been done before.

Since one-cluster states form building blocks for multicluster states, see Part I of this thesis, we first investigated the existence and stability properties of one-cluster states. Here, we introduced a novel type of phase-locked states for complex networks, namely local splay states, and showed that this class of states is nonempty for any nonlocal ring base topology. In particular, we proved that rotating-wave as well as antipodal states are always phase-locked solutions. Compared with the case of a global base topology, the different clusters of local splay type on a nonlocal ring structure can possess different collective frequencies. In addition, we proved that local splay cluster are always global splay cluster. This statement relates, therefore, local with global (with respect to "spatial" structures in the network) properties of solutions.

The stability features of rotating-wave states were studied numerically and analytically. The comparison of both approaches resulted in a very good agreement. Due to the analytic findings for rotating-wave states on a nonlocal ring, we were able to describe their stability depending on the coupling range $P$ and the wavenumber $k$. The limiting case of global coupling, i.e. $P=N / 2$, was shown to be in agreement with the results presented in Chapter 4.

An interesting feature of the system's behavior are solitary states. They had been previously found to emerge in the Kuramoto-Sakaguchi model with inertia [JAR18]. In this article, we showed that solitary states are born in a homoclinic bifurcation and can be (de)stabilized in a pitchfork bifurcation of periodic orbits. In order to show this, a two-dimensional effective model was derived governing the dynamics of solitary states. In contrast to the Kuramoto-Sakaguchi model with inertia, we observed a much more complicated bifurcation behavior. In particular, three different solitary states are created due to two individual homoclinic bifurcations. Two of these three solitary states, however, are unstable and bifurcate together with the stable solitary states in a subcritical pitchfork bifurcation of periodic orbits.

Our results highlight the delicate interplay between adaptivity and complexity of the network structure. Since this interplay has been rarely investigated from the mathematical viewpoint, so far, this work raises many questions for future research which could be conducted for different network structures beyond nonlocal rings, other dynamical models for the local dynamics, nonidentical units or different adaptation rules.

In Chapter 7, we studied the stability of the synchronous state for a complex network of adaptively coupled oscillators. For this, the well-known master stability approach was generalized for networks with adaptive and hence time evolving coupling weights. The master stability approach is already well established and was generalized for various applications, e.g., to understand the stability of cluster synchronous states [DAH12, PEC14, SOR16a] or to study systems with single or distributed delays [SOR07, CHO09, FLU10b, DAH11b, HEI11, KYR14, 
WIL14, LEH15b]. Despite these advances, the novel extension towards adaptive networks can be regarded as non-standard. So far all generalizations were introduced for a static topological structure. However, in the case of an adaptive network the topological structure is non-constant in time which changes the theoretical approach entirely.

The master stability approach was further applied to the paradigmatic model of adaptively coupled phase oscillators. We illustrated several forms for the master stability function with respect to different adaptation rules, i.e., different values for the control parameter $\beta$. Remarkably, the emergence of bounded regions that would lead to stable synchronous dynamics was observed in the master stability function. These bounded regions were called stability islands. We described the structure and formation of stability islands via a cubic curve and derived an existence criterion. Additionally, we analytically provided sufficient conditions for the system parameters which imply the stability of the synchronous state on a undirected background network structure.

Afterwards, the implications of the stability islands were further explored. Using an adiabatic continuation of the synchronous state with respect to the coupling constant $\sigma$, we obtained that the presence of a stability island gives rise to the emergence of multicluster states and chimera-like states. The stable existence of certain multiclusters and chimera-like states were shown numerically and analytically explained using the master stability function. Thus, the findings do not only complement the results obtained in Chapters 5 and 6 . They also underline the subtle interplay between cluster size and network structure for the stability of complex synchronization patterns.

We showed how the generalized master stability approach is used to understand the mechanisms behind the formation of frequency clusters. Another field of application could be the analysis of synchronous states in real-world neuronal systems with synaptic plasticity. Such systems have been discussed recently in order to study pathological diseases such as tinnitus [TAS12a]. However, the connectivity structure of the neuronal system was predefined and fixed in the study [TAS12a]. Our master stability approach for adaptive networks is not restricted to a particular complex network. Thus, the new method could be used to introduce a generalized approach in order to understand unwanted neural synchronization in complex neuronal networks with synaptic plasticity.

In Chapter 8, we proposed a concept to induce diverse partial synchronization patterns (phase clusters) in adaptively coupled multilayer phase oscillator networks. While adaptive networks have recently attracted a lot of attention in the fields of neuro- and social sciences, biology, engineering, and other disciplines, and multilayer networks are a paradigm for real-world complex networks, little had been known about the interplay of multilayer structures and adaptivity. We aimed to fill this gap within a rigorous framework of theoretical analysis and computer simulations. We showed that multiplexing in a multilayer with symmetry can induce various stable phase cluster states like splay states, antipodal states, and double antipodal states, in a situation where they are not stable or do not even exist in the single layer. Further, we developed a novel method for the analysis of Laplacian matrices of duplex networks which allows for insight into the spectral structure of these networks, and can easily be generalized to more than two layers. This new approach of multiplex decomposition has a broad range of applications to physical, biological, socio-economic, and technological systems, ranging from plasticity in neurodynamics or the dynamics of linear diffusive systems [GOM13, SOL13a] to 
generalizations of the master stability approach [PEC98, TAN19] for adaptive networks. We have used the multiplex decomposition to provide analytic results for the stability of lifted states in the multilayer system. As local dynamics we used the paradigmatic Kuramoto phase oscillator model, supplemented by adaptivity of the link strengths with a phase lag parameter which can model a whole range of adaptivity rules from Hebbian via spike timing-dependent plasticity to anti-Hebbian.

While several applications of the multiplex decomposition were presented in this thesis, many other possible fields of application remain untouched. An intriguing opportunity to apply our new methodology arises in the control of power grid networks. For these particular realworld networks, it is of great importance to retain synchronization when single nodes or lines fail [WIT16]. Multiplexing with a control layer could be used to establish an efficient way to stabilize power grids against topological changes [TOT20]. A control layer approach might also be used to prevent the power grids from the cascading of line failures due to overloads [SCH18i].

As we saw in this thesis, adaptive networks of phase oscillators give rise to a plethora of dynamical scenarios. These scenarios include complete and cluster synchronization, frequency clustering, solitary states, and chimera-like states. The reason behind this variety of states is the adaptivity of the coupling weights. This flexibility in the coupling structure allows each individual oscillator to adapt indirectly its own frequency and thus leads to the formation of different frequency clusters. Frequency adaptation is also a property of Kuramoto phase oscillators with inertia that is a key model in the theory of power grids. The introduction of inertia enables the oscillators to adapt their frequencies directly. Remarkably, frequency clustered states are also widely observed in networks of Kuramoto phase oscillators with inertia [OLM15a, JAR18, MEH18, TUM18, TAH19, TUM19a, HEL20, TOT20]. Frequency adaptation is present but implemented differently in both systems. Therefore, an open and very intriguing question arises as to whether it is possible to relate the models of phase oscillators on adaptive networks to the models of phase oscillators with inertia. 
Appendix 



\section{Proof of results from the main text}

\section{A.1 One-cluster states on globally coupled adaptive networks}

Here we provide a proof of Proposition 4.1.1. We first need a preliminary lemma.

Lemma A.1.1 For a phase-locked solution $\phi(t), R_{2}(\phi(t))=1$ for all $t$ if and only if $\boldsymbol{\phi}(t)$ is either an in-phase or an anti-phase synchronous solution.

Proof. As follows from table 4.1, $R_{2}(\mathbf{a})=\frac{1}{N}\left|\sum_{j=1}^{N} e^{\mathrm{i} 2 a_{j}}\right|=1$ for all in-phase and anti-phase solutions. Let us show the opposite. If $R_{2}(\mathbf{a})=1$, then $e^{\mathrm{i} 2 a_{j}}=e^{\mathrm{i} 2 a_{1}}=1$ for all $j$, since $\left|\sum_{j=1}^{N} e^{\mathrm{i} 2 a_{j}}\right| \leq$ $\sum_{j=1}^{N}\left|e^{\mathrm{i} 2 a_{j}}\right|=N$. Hence, $a_{j} \in\{0, \pi\}$. The latter means that the phase-locked solution is either in-phase, if all $a_{j}$ have the same values, or anti-phase otherwise.

Now we present the proof of Proposition 4.1.1.

Proof. Substituting (4.3)-(4.4) into (2.25)-(2.26) we obtain $\dot{\kappa}_{i j}=0$ and

$$
\begin{aligned}
\dot{\phi}_{i}(t)=\Omega & =\frac{1}{N} \sum_{j=1}^{N} \sin \left(a_{i}-a_{j}+\beta\right) \sin \left(a_{i}-a_{j}+\alpha\right) \\
& =\frac{1}{2} \cos (\alpha-\beta)-\frac{1}{2} \mathfrak{R}\left(e^{-\mathrm{i}\left(2 a_{i}+\alpha+\beta\right)} Z_{2}(a)\right) .
\end{aligned}
$$

Therefore (4.3)-(4.4) are solutions if and only if the expression on the right-hand side of the equation (A.1) is independent of the oscillators index $i=1, \ldots, N$. In particular, for any choice of $a_{i}$ the complex second order parameter is either zero or can be written as $Z_{2}(\mathbf{a})=R_{2}(\mathbf{a}) e^{-\mathrm{i} \gamma}$. Thus, according to (A.1) $a_{i}$ has to be such that $R_{2}(\boldsymbol{a})=0$ or $\cos \left(2 a_{i}+\alpha+\beta+\gamma\right)$ is independent of $i$. For any $\alpha, \beta$ and $\gamma$ the latter requirement is equivalent with $2 a_{i} \in\{0,-2(\alpha+\beta+\gamma)\}$. Here, we made use of the phase-shift symmetry of (2.25)-(2.26) by setting $2 a_{1}=0$. Due to the definition of the complex order parameter the value for $\gamma$ depends on the choice of the phase lags $a_{i}$. Assuming that one fraction $q_{1}=Q_{1} / N$ of the oscillators have $2 a_{i}=0$ with $Q_{1} \in\{1, \ldots, N-1\}$ and the other fraction of oscillators $q_{2}=1-q_{1}$ have $2 a_{i}=-2(\alpha+\beta+\gamma)$ one obtains

$$
q_{1}+q_{2} e^{-\mathrm{i} 2(\alpha+\beta)} e^{-\mathrm{i} 2 \gamma}=R_{2}(\mathbf{a}) e^{-\mathrm{i} \gamma} .
$$

which is equivalent to the equations

$$
\begin{aligned}
& q_{1} \cos (\gamma)+q_{2} \cos (\gamma+2 \vartheta)=R_{2}(\mathbf{a}), \\
& q_{1} \sin (\gamma)-q_{2} \sin (\gamma+2 \vartheta)=0 .
\end{aligned}
$$

with $\vartheta=\alpha+\beta$. Here, the first equation gives the value for the second order parameter while the second equation determines $\gamma$. A special solution can be given if we set $q_{2}=0$, equivalently 
$q_{1}=1$. Then $\gamma=0$ and $\gamma=\pi$ would solve the equation above and thus $2 a_{i}=0$ for all $i=1, \ldots, N$. Note that, for both values of $\gamma$ the value for the complex second order parameter coincide $Z_{2}(\mathbf{a})=1$. This solution corresponds to $R_{2}(\mathbf{a})=1$. In any other case the last equation can be written in the form $\sin (\gamma-v)=0$ which has two solutions $\gamma=v, v+\pi$. Both solution would coincide while determining $2 a_{i}$. Writing (A.2) as

$$
\frac{1}{C}\left(\left(q_{1}-\left(1-q_{1}\right) \cos (2 \vartheta)\right) \sin (\gamma)-\left(1-q_{1}\right) \sin (2 \vartheta) \cos (\gamma)\right)=0
$$

where the normalization constant $C$ is defined as

$$
C=\sqrt{\left(q_{1}-\left(1-q_{1}\right) \cos (2 \vartheta)\right)^{2}+\left(1-q_{1}\right)^{2} \sin ^{2}(2 \vartheta)},
$$

yields the equations

$$
\begin{aligned}
& \sin (v)=\frac{\sin (2 \vartheta)}{\sqrt{\left(\frac{q_{1}}{1-q_{1}}\right)^{2}+1-2 \frac{q_{1}}{1-q_{1}} \cos (2 \vartheta)}} \\
& \cos (v)=\frac{q_{1}-\left(1-q_{1}\right) \cos (2 \vartheta)}{\sqrt{\left(1-q_{1}\right)^{2}+q_{1}^{2}-2 q_{1}\left(1-q_{1}\right) \cos (2 \vartheta)}} .
\end{aligned}
$$

Therefore, considering the inverse function arcsin : $[-1,1] \rightarrow[-\pi / 2, \pi / 2]$ applied to (A.3) determines $v$ to be either $v^{\prime}$ or $\pi-v^{\prime}$, where $v^{\prime}:=\arcsin (\sin (v))$ and $\sin (v)$ as given in (A.3). The second equation for $\cos (v)$ then fixes $v$ to take one of the values. Thus, $\gamma$ exists and is unique for every $q_{1} \in[0,1)$. The Proposition is proved by taking into account that for a finite number $N$ of oscillators $q_{1}$ takes values in the range from $1 / N$ to $(N-1) / N$ and defining $\gamma:=-\psi-\vartheta$.

\section{A.2 Stability of one-cluster states on globally coupled networks}

In order to study the local stability of one-cluster solutions described in Sec. 4.1, we linearise the system of differential equations (2.25)-(2.26) around the phase locked states described by $\phi_{i}=\Omega t+a_{i}$ and $\kappa_{i j}=-\sin \left(a_{i}-a_{j}+\beta\right)$. We obtain the following linearised system

$$
\begin{array}{r}
\frac{d}{d t} \delta \phi_{i}=\frac{1}{2 N} \sum_{j=1}^{N} \sin (\beta-\alpha)\left(\delta \phi_{i}-\delta \phi_{j}\right)+\frac{1}{2 N} \sum_{j=1}^{N} \cos \left(2\left(a_{i}-a_{j}\right)+\alpha+\beta\right)\left(\delta \phi_{i}-\delta \phi_{j}\right) \\
-\frac{1}{N} \sum_{j=1}^{N} \sin \left(a_{i}-a_{j}+\alpha\right) \delta \kappa_{i j}
\end{array}
$$

and

$$
\frac{d}{d t} \delta \kappa_{i j}=-\epsilon\left(\delta \kappa_{i j}+\cos \left(a_{i}-a_{j}+\beta\right)\left(\delta \phi_{i}-\delta \phi_{j}\right)\right)
$$


Note that this set of equations can be brought into the following block form

$$
\frac{\mathrm{d}}{\mathrm{d} t}\left(\begin{array}{c}
\delta \phi \\
\delta \kappa
\end{array}\right)=\left(\begin{array}{cc}
A & B \\
C & -\epsilon \mathbb{I}_{N^{2}}
\end{array}\right)\left(\begin{array}{c}
\delta \phi \\
\delta \kappa
\end{array}\right)
$$

where $(\delta \boldsymbol{\phi})^{T}=\left(\delta \phi_{1}, \ldots, \delta \phi_{N}\right),(\delta \kappa)^{T}=\left(\delta \kappa_{11}, \ldots, \delta \kappa_{1 N}, \delta \kappa_{21}, \ldots, \delta \kappa_{N N}\right), B=\left(\begin{array}{lll}B_{1} & \cdots & B_{N}\end{array}\right)$, $C=\left(\begin{array}{c}C_{1} \\ \vdots \\ C_{N}\end{array}\right)$, and $A, B_{n}, C_{n}$ are $N \times N$ matrices with $n=1, \ldots, N$. The elements of the block matrices read

$$
\begin{aligned}
a_{i j} & = \begin{cases}-\frac{1}{2} \sin (\alpha-\beta)-\frac{1}{N} \sin (\beta) \cos (\alpha) \\
+\frac{1}{2 N} \sum_{k=1}^{N} \sin \left(2\left(a_{i}-a_{k}\right)+\alpha+\beta\right), \\
\frac{1}{2 N}\left(\sin (\alpha-\beta)-\sin \left(2\left(a_{i}-a_{j}\right)+\alpha+\beta\right)\right), \quad i \neq j\end{cases} \\
b_{i j ; n} & = \begin{cases}-\frac{1}{N} \sin \left(a_{n}-a_{j}+\alpha\right), & i=n \\
0, & \text { otherwise }\end{cases} \\
c_{i j ; n} & = \begin{cases}0, & j=n, i=j \\
-\epsilon \cos \left(a_{n}-a_{i}+\beta\right), & j=n, i \neq j \\
\epsilon \cos \left(a_{n}-a_{i}+\beta\right), & j \neq n, i=j \\
0, & \text { otherwise }\end{cases}
\end{aligned}
$$

Throughout this section we will make use of the Schur complement [BOY04, LIE15] in order to simplify characteristic equations as it has been introduced Eq. (4.11) in Chapter 4. In particular, the determinant of the $p \times p$ matrix $M=\left(\begin{array}{ll}A & B \\ C & D\end{array}\right)$ can be simplified to the following expression by using (4.11)

$$
\operatorname{det}(M)=\operatorname{det}\left(A-B D^{-1} C\right) \cdot \operatorname{det}(D)
$$

This result is important for the subsequent stability analysis. So far, we have found the Lyapunov coefficients for the rotating-wave states, see Corollary 4.2.3. The following two Lemmata are needed to describe the stability of all antipodal, 4-phase cluster, and double antipodal states as well.

Lemma A.2.1 Suppose $M$ is a block square matrix of the form

$$
M=\left(\begin{array}{cc}
A & m_{1} \hat{1}_{p, q} \\
m_{2} \hat{1}_{q, p} & B
\end{array}\right)
$$

where $A$ is a circulant $p \times p$ matrix, $B$ is a circulant $q \times q$ matrix, $\hat{1}_{p, q}$ is $p \times q$ where all entries are 1 
and $m_{1}, m_{2} \in \mathbb{R}$. Then the eigenvector-eigenvalue pairs are given by

$$
\begin{aligned}
& \left(\lambda_{k}^{0}, \ldots, \lambda_{k}^{p-1}, 0, \ldots, 0\right)^{T}, \mu_{k}=\sum_{l=0}^{p-1} a_{1(1+l)} \lambda_{k}^{l} \\
& \left(0, \ldots, 0, \rho_{l}^{0}, \ldots, \rho_{l}^{q-1}\right)^{T}, v_{l}=\sum_{l=0}^{q-1} b_{1(1+l)} \rho_{l}^{l} \\
& \left(1, \ldots, 1, a_{1}, \ldots a_{1}\right)^{T}, \bar{\mu}=\mu_{0}+m_{1} q a_{1} \\
& \left(1, \ldots, 1, a_{2}, \ldots, a_{2}\right)^{T}, \bar{v}=\mu_{0}+m_{1} q a_{2}
\end{aligned}
$$

with $\lambda_{k}=e^{\mathrm{i} \frac{2 \pi}{p} k}$ and $\rho_{l}=e^{\mathrm{i} \frac{2 \pi}{q} l}$ for $k=1, \ldots, p-1$ and $l=1, \ldots, q-1$, respectively, and $a_{1}$ and $a_{2}$ solve the equation

$$
a^{2}+\frac{\mu_{0}-v_{0}}{m_{1} q} a-\frac{m_{2} p}{m_{1} q}=0
$$

with $\mu_{0}=\sum_{j=1}^{p} a_{1 j}$ and $v_{0}=\sum_{j=1}^{q} b_{1 j}$.

Proof. We can prove the Lemma by direct calculation and find

$$
\begin{aligned}
M\left(\lambda_{k}^{0}, \ldots, \lambda_{k}^{p-1}, 0, \ldots, 0\right)^{T} & =\left(A\left(\lambda_{k}^{0}, \ldots, \lambda_{k}^{p-1}\right)^{T} m_{2} \sum_{l=0}^{p-1} \lambda_{k}^{l}\right) \\
& =\mu_{k}\left(\lambda_{k}^{0}, \ldots, \lambda_{k}^{p-1}, 0, \ldots, 0\right)^{T}
\end{aligned}
$$

Here, we use that $A$ is a circulant matrix and that $\sum_{l=0}^{p-1} \lambda_{k}^{l}=0$ for all $k=1, \ldots, p-1$. Analogous arguments hold for (A.8). The last two eigenvector-eigenvalue pairs (A.9)-(A.10) can be obtained by

$$
\begin{aligned}
M(1, \ldots, 1, a, \ldots, a)^{T} & =\left(\begin{array}{c}
A(1, \ldots, 1)^{T}+m_{1} q a(1, \ldots, 1)^{T} \\
\frac{m_{2} p}{a}(1, \ldots, 1)^{T}+a B(1, \ldots, 1)^{T}
\end{array}\right) \\
& =\left(\begin{array}{c}
\mu_{0}+m_{1} q a \\
\frac{m_{2} p}{a}+v_{0}
\end{array}\right)(1, \ldots, 1, a, \ldots, a)^{T}
\end{aligned}
$$

which solves the eigenvalue problem if $a$ is chosen to be either $a_{1}$ or $a_{2}$.

Lemma A.2.2 Suppose we have a phase locked state with phases $a_{i} \in[0,2 \pi)$. Then, the solution for the characteristic equations corresponding to the linearised system (4.9)-(4.10) are given by $\lambda=-\epsilon$ with multiplicity $N^{2}-N$ and by the solution of the following set of equations

$$
\operatorname{det}\left(\left(A-\lambda \mathbb{I}_{N}\right)(\epsilon+\lambda)+B C\right)=0 \text {. }
$$

Proof. Applying Schur's decomposition (4.11) to the linearised system in the block form (A.6) yields the result.

Proposition A.2.3 Suppose we have a state with phases $a_{i} \in\{0, \pi, \psi, \psi+\pi\}$ where $i=1, \ldots, N$. Further set $q_{1}=Q_{1} / N$ and $q_{2}=Q_{2} / N$, where $Q_{1}$ and $Q_{2}$ denote the numbers of phases which are either 0 
or $\pi$ and $\psi$ or $\psi+\pi$, respectively. Then, the linear system (4.9)-(4.10) possesses the following set $L$ of eigenvalues

$$
L=\left\{(0)_{1},(-\epsilon)_{(N-1) N+1},\left(\lambda_{1}\right)_{N_{1}-1},\left(\lambda_{2}\right)_{N_{1}-1},\left(\vartheta_{1}\right)_{N_{2}-1},\left(\vartheta_{2}\right)_{N_{2}-1},\left(\rho_{1}\right)_{1},\left(\rho_{2}\right)_{1}\right\}
$$

where $\lambda_{1}$ and $\lambda_{2}$ solve

$$
\begin{aligned}
\lambda^{2}+\frac{1}{2}\left(\sin (\alpha-\beta)-q_{1} \sin (\alpha+\beta)-q_{2} \sin (-2 \psi+\alpha+\beta)+2 \epsilon\right) \lambda & \\
& -\epsilon q_{1} \sin (\alpha+\beta)-\epsilon q_{2} \sin (-2 \psi+\alpha+\beta)=0,
\end{aligned}
$$

$\vartheta_{1}$ and $\vartheta_{2}$ solve

$$
\begin{aligned}
\vartheta^{2}+\frac{1}{2}\left(\sin (\alpha-\beta)-q_{1} \sin (2 \psi+\alpha+\beta)-q_{2} \sin (\alpha+\beta)+2 \epsilon\right) \vartheta & \\
& -\epsilon q_{1} \sin (2 \psi+\alpha+\beta)-\epsilon q_{2} \sin (\alpha+\beta)=0,
\end{aligned}
$$

as well as $\rho_{1}$ and $\rho_{2}$ solve

$$
\begin{aligned}
\rho^{2}+\frac{1}{2}\left(\sin (\alpha-\beta)-q_{1} \sin (2 \psi+\alpha+\beta)-q_{2} \sin (\right. & -2 \psi+\alpha+\beta)+2 \epsilon) \rho \\
& -\epsilon q_{1} \sin (2 \psi+\alpha+\beta)-\epsilon q_{2} \sin (-2 \psi+\alpha+\beta)=0 .
\end{aligned}
$$

The multiplicities for each eigenvalue are given as subscripts.

Proof. For an arbitrary solution of the form $\phi_{i}=\Omega t+a_{i}$ we consider the linearized system (4.9)(4.10) in the block form (A.6) and apply Lemma A.2.2. The elements of the second term $D:=B C$ of Schur's complement are then

$$
d_{i j}=-\frac{\epsilon}{2 N}\left(\sin (\alpha-\beta)+\sin \left(2\left(a_{i}-a_{j}\right)+\alpha+\beta\right)\right)
$$

if $i \neq j$ and

$$
d_{i i}=\epsilon\left(\frac{1}{2} \sin (\alpha-\beta)-\frac{1}{N} \sin (\alpha) \cos (\beta)+\frac{1}{2 N} \sum_{j=1}^{N} \sin \left(2\left(a_{i}-a_{j}\right)+\alpha+\beta\right)\right) .
$$

Defining the matrix $M:=\left(A-\lambda \mathbb{I}_{N}\right)(\epsilon+\lambda)+D$ we get

$$
m_{i j}= \begin{cases}-\lambda^{2}+\left(a_{i i}-\epsilon\right) \lambda+\epsilon a_{i i}+d_{i i} & i=j \\ \lambda a_{i j}+\epsilon a_{i j}+d_{i j} . & i \neq j\end{cases}
$$

Using the assumption for the phases $a_{i}$, then one group of oscillators (group $I$ ) have $a_{i} \in\{0, \pi\}$ and the remaining $Q_{2}$ oscillators (group $I I$ ) have $a_{i}=\{\psi, \psi+\pi\}$. Putting this into the definition 
of $m_{i j}$, we find that the whole square matrix $M$ can be written as

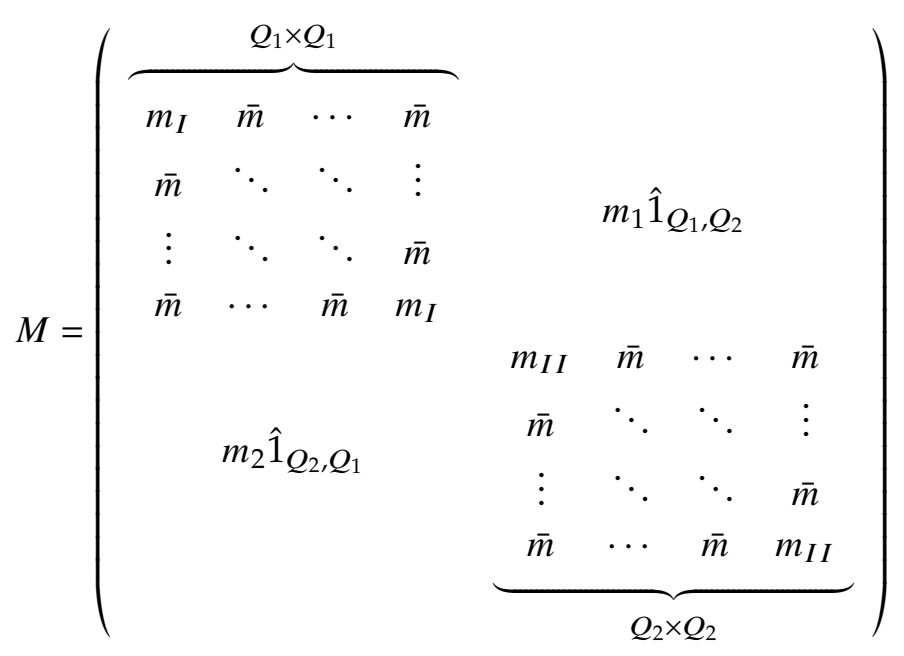

where $m_{1}, \bar{m}, m_{I}$, and $m_{I I}$ are real values which depend on all the system parameters $\alpha, \beta, \epsilon$ and additionally on $\psi$ and $\lambda$. Note that all diagonal blocks are circulant matrices. The determinant is invariant under basis transformations which is why we diagonalize the matrix $M$ and therewith derive equations for the values $\lambda$. In order to do so, we look for the eigenvalues of $M$ determined by the characteristic equation

$$
\operatorname{det}\left(M-\mu \mathbb{I}_{N}\right)=0 \text {. }
$$

Due to the structure of $M$ we can apply Lemma A.2.1 and find the following set of eigenvalues

$$
\begin{aligned}
\mu_{k}=-\lambda^{2}-\frac{1}{2}\left(\sin (\alpha-\beta)-q_{1} \sin (\alpha+\beta)-q_{2} \sin (-2 \psi+\alpha\right. & +\beta)+2 \epsilon) \lambda \\
& +\epsilon q_{1} \sin (\alpha+\beta)+\epsilon q_{2} \sin (-2 \psi+\alpha+\beta)
\end{aligned}
$$

for $k=1, \ldots, Q_{1}-1$. Analogously, we obtain the equations for $v_{k}\left(k=1, \ldots, Q_{2}-1\right)$ where $m_{I}$ is substituted with $m_{I I}$. The two other eigenvalue are given by $\bar{\mu}=\mu_{0}+m_{1} Q_{2} a_{1}$ and $\bar{v}=$ $\mu_{0}+m_{1} Q_{2} a_{2}$, respectively, where

$$
\mu_{0}=m_{I}+\left(Q_{1}-1\right) \bar{m}
$$

and $a_{1,2}$ are given by

$$
a^{2}+\frac{\left(m_{I}-m_{I I}\right)+\left(Q_{1}-Q_{2}\right) \bar{m}}{m_{1} Q_{2}} a-\frac{m_{2} Q_{1}}{m_{1} Q_{2}}=0 .
$$

Considering the row sums of $M$ we find that all agree with $-\lambda^{2}-\epsilon \lambda$ and therefore $\bar{\mu}=-\lambda^{2}-\epsilon \lambda$. Resulting from this $a_{1}=-\left(\lambda^{2}+\epsilon \lambda+\mu_{0}\right) / m_{1} Q_{2}=1$. Hence,

$$
a_{2}=\frac{\left(m_{I I}-m_{I}\right)+\left(Q_{2}-Q_{1}\right) \bar{m}}{m_{1} Q_{2}}-1
$$


and we find

$$
\begin{aligned}
\bar{v}= & m_{I I}+\left(Q_{2}-1\right) \bar{m}-m_{1} Q_{2} \\
= & -\lambda^{2}-\frac{1}{2}\left(\sin (\alpha-\beta)-q_{1} \sin (2 \psi+\alpha+\beta)-q_{2} \sin (-2 \psi+\alpha+\beta)+2 \epsilon\right) \lambda \\
& +\epsilon q_{1} \sin (2 \psi+\alpha+\beta)+\epsilon q_{2} \sin (-2 \psi+\alpha+\beta)
\end{aligned}
$$

After diagonalizing the matrix $M$ the determinant can be easily written as

$$
\operatorname{det}(M)=\bar{\mu} \cdot \mu_{1} \cdots \mu_{N_{1}-1} \cdot \bar{v} \cdot v_{1} \cdots v_{N_{2}-1} .
$$

Therewith, finding $\lambda^{\prime} s$ such that at least one of the eigenvalues of $M$ vanishes solves the initial eigenvalue problem.

We will now sum up the results with the following corollaries

Corollary A.2.4 The set of eigenvalues of the linearised system (4.9)-(4.10) around all antipodal states with $a_{i} \in\{0, \pi\}$ agrees with the set $L$ in Prop. 4.2.2 for rotating-wave states with $k=0, N / 2$.

Proof. Put $Q_{2}=0$ in Prop. A.2.3, then there is only the equation for $\lambda$ left.

Corollary A.2.5 The set of eigenvalues to the linearised system (4.9)-(4.10) around all 4-phase-cluster states with $a_{i} \in\{0, \pi / 2, \pi, 3 \pi / 2\}$ and $R_{2}(a)=0$ agrees with the set $L$ in Prop. 4.2.2 for 4-rotating-wave states.

Proof. The requirement $R_{2}(\boldsymbol{a})=0$ yields $Q_{1}=Q_{2}$. The statement of this proposition follows by using Prop. A.2.3.

Corollary A.2.6 For all $\alpha$ and $\beta$ the double antipodal states are unstable.

Proof. Suppose the polynomial equation $p(x)=x^{2}+a x+b=0$. This equation has two negative roots if and only if $b>0$ and $a>0$ meaning that $p(0)>0$ and the vertex of the parabola is at $x<0$, respectively. In order to have stable double antipodal states these two conditions have to be met by all three equations for $\lambda, \vartheta$ and $\rho$ in Proposition A.2.3. From the condition on the existence of double antipodal states (4.5) we find $q_{1} \sin (2 \psi+\alpha+\beta)+q_{2} \sin (-2 \psi+\alpha+\beta)=-\sin (\alpha+\beta)$. With this assumption on the quadratic equation and the latter equation, we find the following two necessary conditions for the stability of double antipodal states, (1) $q_{1} \sin (2 \psi+\alpha+\beta)+$ $q_{2} \sin (\alpha+\beta)>0$ and (2) $q_{1} \sin (2 \psi+\alpha+\beta)+q_{2} \sin (\alpha+\beta)<0$. The two condition cannot be equally fulfilled. 


\section{A.3 Multicluster states of splay type}

Proof of Proposition 5.2.1. We prove by direct substitution. Plugging (5.4) and (5.5) into (2.26) the identity is obtained. Further, substituting (5.4) and (5.5) into (2.26) we obtain

$$
\begin{aligned}
\Omega_{\mu} & =\frac{1}{2 N} \sum_{\nu=1}^{M} \rho_{\mu \nu} \sum_{j=1}^{N_{\mu}}\left(\cos \left(\alpha-\beta+\psi_{\mu \nu}\right)-\cos \left(2\left(\Delta \Omega_{\mu \nu} t+a_{i, \mu}-a_{j, v}\right)+\alpha+\beta-\psi_{\mu \nu}\right)\right) \\
& =\sum_{\nu=1}^{M} \rho_{\mu \nu}\left(\frac{n_{v}}{2} \cos \left(\alpha-\beta+\psi_{\mu \nu}\right)-\frac{1}{2} \mathfrak{R}\left(e^{-\mathrm{i}\left(2 \Delta \Omega_{\mu \nu} t+2 a_{i, \mu}+\alpha+\beta-\psi_{\mu \nu}\right)} Z_{2}\left(\mathbf{a}_{\nu}\right)\right)\right) .
\end{aligned}
$$

If $Z_{2}\left(\mathbf{a}_{\mu}\right)=0$ for all $\mu=1, \ldots, M$ then

$$
\Omega_{\mu}=\frac{1}{N} \sum_{\nu=1}^{M} \sum_{j=1}^{N_{\mu}} \rho_{\mu \nu} \cos \left(\alpha-\beta+\psi_{\mu \nu}\right)
$$

which agrees with the system (5.6) for the frequencies $\Omega_{\mu}$. On the contrary, assume that the multicluster phase-locked solution (5.4) and (5.5) solve the equation (2.25). Analog to Proposition 4.1.1 $\sum_{\nu=1}^{M} \rho_{\mu \nu} \mathfrak{R}\left(e^{-\mathrm{i}\left(2 \Delta \Omega_{\mu \nu} t+2 a_{i, \mu}+\alpha+\beta-\psi_{\mu \nu}\right)} Z_{2}\left(\mathbf{a}_{\nu}\right)\right)$ has to be independent of the oscillator index $i=1, \ldots, N_{\mu}$ and $t \in \mathbb{R}$ for all $\mu=1, \ldots, M$. Take any $\mu=1, \ldots, M$ and suppose $Z_{2}\left(\mathbf{a}_{\nu}\right) \neq 0$ for $v \in A$ with $A \subseteq\{1, \ldots, M\}$. Then

$$
\begin{aligned}
& \sum_{\nu=1}^{M} \rho_{\mu \nu} \mathfrak{R}\left(e^{-\mathrm{i}\left(2 \Delta \Omega_{\mu \nu} t+2 a_{i, \mu}+\alpha+\beta-\psi_{\mu \nu}\right)} Z_{2}\left(\mathbf{a}_{\nu}\right)\right)= \\
& \sum_{\nu \in A} \rho_{\mu \nu}\left(R_{2}\left(\mathbf{a}_{\nu}\right) \cos \left(2 \Delta \Omega_{\mu \nu} t+2 a_{i, \mu}+\alpha+\beta-\psi_{\mu \nu}+\gamma_{\nu}\right)\right)
\end{aligned}
$$

where $\gamma_{v}$ is defined as in Proposition 4.1.1, see type 2 or 3. For fixed $\mu$ all frequency differences $\Delta \Omega_{\mu \nu}$ differ due to the assumption that the frequencies $\Omega_{\mu}$ are all pairwise different. This is why only terms with $\Delta \Omega_{\mu \nu}$ and $\Delta \Omega_{\mu \nu^{\prime}}=-\Delta \Omega_{\mu \nu}\left(v, v^{\prime} \in A\right)$ are candidates to compensate each other in the right hand side of (A.11) to give a constant value for $\Omega_{\mu}$. Therefore, the number of clusters with $Z_{2}\left(\mathbf{a}_{v}\right) \neq 0$ excluding the $\mu$-th cluster which is under consideration has to be even, i.e., $|A \backslash\{\mu\}|$ even for all $\mu=1, \ldots, M$. This already yields that $|A|$ odd and $A=\{1, \ldots, M\}$. Consider now $\mu$ such that $\Omega_{\mu}=\min _{v \in 1, \ldots, M} \Omega_{v}$. Then for every other $v \in\{1, \ldots, M\}$ with $\Delta \Omega_{\mu \nu}<0$ there has to be $v^{\prime} \in\{1, \ldots, M\}$ so that $-\Delta \Omega_{\mu \nu}=\Delta \Omega_{\mu v^{\prime}}=\Omega_{\mu}-\Omega_{v^{\prime}}$. Hence, $\Omega_{v^{\prime}}<\Omega_{\mu}$ which contradicts that $\Omega_{\mu}=\min _{v \in 1, \ldots, M} \Omega_{v}$. Therefore, for this choice of $\mu \in 1, \ldots, M$ the expression in (A.11) cannot be constant contradicting the assumption made in the beginning.

\section{A.4 Asymptotic expansions of multicluster states}

In this section we give an analytic description of multicluster solutions in terms of an asymptotic expansion. We consider therefore the expansion of $r$-th order together with a multi-time scale 
ansatz [VER06] as given in 5.11

$$
\begin{aligned}
\phi_{i, \mu}^{(r)}(\epsilon, t) & :=\Omega_{\mu}^{(r)}\left(\tau_{0}, \ldots, \tau_{r}\right)+a_{i, \mu}+\sum_{l=1}^{r} \epsilon^{l} p_{i, \mu ; l}(t) \\
\kappa_{i j, \mu \nu}^{(r)}(\epsilon, t) & :=\sum_{l=0}^{r} \epsilon^{l} k_{i j, \mu v ; l}(t)
\end{aligned}
$$$$
\begin{aligned}
\mu, v & =1, \ldots, M \\
i, j & =1, \ldots, N_{\mu}
\end{aligned}
$$

where $\Omega_{\mu}^{(r)} \in C^{1}\left(\mathbb{R}^{r+1}\right)$ is a function depending on the multi-time scales $\tau_{l}:=\epsilon^{l} t$. We show under under which conditions this expansion describes the time evolution for the system (2.25)-(2.26).

The section is organized as follows. We first introduce some notations and prove some technical lemmas that will help us to prove the main result in Proposition 5.3.1. For ease of notation, for the remainder of the section indices are used as follows. Small Latin letters $i, j$ in the subscript are oscillator indices while Greek letters $\mu, v$ represent cluster indices. These are separated by a comma. Two further indices, separated by semicolon, are the coefficient index and the mode index, respectively. The indices in superscript are either powers or the order for the expansion which are then written in parenthesis.

The following definition is introduced to handle the order of approximation.

Definition A.4.1 Let $f: \mathbb{R} \times \mathbb{R} \rightarrow \mathbb{R}$ and $g: \mathbb{R} \times \mathbb{R} \rightarrow \mathbb{R}$ real functions. We define the following notations:

1. $f(\epsilon, t) \in O(g(\epsilon, t))$ as $\epsilon \rightarrow 0$ on the interval $I \subseteq \mathbb{R}$ if for any $t \in I$ there exist $C(t)>0$ and $\epsilon_{0}(t)>0$ such that $|f(\epsilon, t)|<C(t)|g(\epsilon, t)|$ for all $\epsilon<\epsilon_{0}(t)$,

2. $f(\epsilon, t) \in o(g(\epsilon, t))$ as $\epsilon \rightarrow 0$ on the interval $I \subseteq \mathbb{R}$ if for any $t \in I$ and all $C>0$ there exist $\epsilon_{0}(t)>0$ such that $|f(\epsilon, t)|<C|g(\epsilon, t)|$ for all $\epsilon<\epsilon_{0}(t)$.

Remark A.4.1 If the constants $C$ and $\epsilon_{0}$ can be chosen independently of $t \in I$ we say that $f(\epsilon, t) \in O(g(\epsilon, t))$ (or $f(\epsilon, t) \in o(g(\epsilon, t)))$ as $\epsilon \rightarrow 0$ uniformly on $I$.

In order to find an expressions for the asymptotic expansion of the coupling weights $\kappa$, we use the concept of the pullback attractor. It is defined as an nonempty, compact and invariant set and well known from the theory of nonautonomous dynamical systems. For our purposes, suppose we know the functions $\phi_{i}(t)$ for all $i=1, \ldots, N$. Then, the differential equations (2.26) is nonautonomous and can be solved explicitly by

$$
\kappa_{i j}(t):=\kappa_{i j, 0} e^{-\epsilon\left(t-t_{0}\right)}-\epsilon \int_{t_{0}}^{t} e^{-\epsilon\left(t-t^{\prime}\right)} \sin \left(\phi_{i}\left(t^{\prime}\right)-\phi_{j}\left(t^{\prime}\right)+\beta\right) \mathrm{d} t^{\prime}
$$

with $\kappa_{i j, 0} \in[-1,1]$ for all $i, j=1, \ldots, N$. For this, the pullback attractor $\mathscr{A}$ is given by the set

$$
\mathscr{A}:=\bigcup_{t \in \mathbb{R}}\left\{(\kappa(t))_{*}\right\}
$$


where

$$
\left(\kappa_{i j}(t)\right)_{*}:=-\lim _{t_{0} \rightarrow-\infty}\left(\epsilon \int_{t_{0}}^{t} e^{-\epsilon\left(t-t^{\prime}\right)} \sin \left(\phi_{i}\left(t^{\prime}\right)-\phi_{j}\left(t^{\prime}\right)+\beta\right) \mathrm{d} t^{\prime}\right)
$$

for all $i, j \in\{1, \ldots, N\}$. Note that (A.14) is the unique bounded solution of (2.26), see Theorem IV.1.1 of [HAL80]. We remark the following important properties. For given functions $\phi_{i}$ the equations (2.26) possess the compact absorbing set $G:=\left\{\kappa_{i j}: \kappa_{i j} \in[-1,1], i, j=1, \ldots, N\right\}$. Hence, the pullback attractor exists, c.f. Theorem 3.18 in [KLO11], and is unique due to Proposition 3.8 [KLO11]. Moreover, $(\kappa(t))_{*}$ is a solution for the nonautonomous system which can be shown by direct computation. We call $\kappa(t))_{*}$ the parametrization of the pullback attractor and use it in order to find an analytic expression for the (pseudo-)multicluster states. Note that we have already seen such parametrizations explicitly in (4.4) and (5.5). For more details regarding nonautonomous systems and the pullback attractor we refer the reader to [RAS06a, KLO11].

We use the following notations for the sake of brevity.

$$
\begin{aligned}
\mathbf{M} & :=\left\{\mathbf{m}=\left(m_{1}, \ldots, m_{M}\right): m_{1}, \ldots, m_{M} \in \mathbb{Z}\right\}, \\
c_{\mathbf{m}} & :=c_{m_{1}, \ldots, m_{M}} \\
\Delta \Omega(\mathbf{m}) & :=\sum_{\mu=1}^{M} m_{\mu} \Omega_{\mu} .
\end{aligned}
$$

Furthermore, we say that two elements $\mathbf{m}, \mathbf{n} \in \mathbf{M}$ are equivalent $\mathbf{m} \sim \mathbf{n}$ if and only if $\Delta \Omega(\mathbf{m})=$ $\Delta \Omega(\mathbf{n})$. The corresponding quotient space is denoted by $\tilde{\mathbf{M}}:=\mathbf{M} / \sim$. If $\Omega_{\mu}$ is considered as frequencies the equivalence relation factors out all resonant linear combinations of those. Let us further define $\tilde{\mathbf{M}}(f)$ as the set of all $\left(m_{1}, \ldots, m_{M}\right)$ such that the function $f$ can be written as $f=\sum_{\mathbf{m} \in \mathbf{M}(f)} c_{\mathbf{m}} e^{\mathrm{i} \Delta \Omega(\mathbf{m}) t}$ for some $c_{\mathbf{m}} \in \mathbb{C}$. Finally, we introduce the shorthand notion $(m \mu n v):=$ $\left(0, \ldots, 0, m_{\mu}, 0, \ldots, 0, m_{v}, 0, \ldots, 0\right)$ with $m_{\mu}=m$ and $m_{v}=n$ for further convenience if only frequencies of two distinguished clusters are considered.

The proof of Proposition 5.3.1 makes use of several lemmas and is presented at the end of this section. Overall, we aim to describe the following particular form for the dynamical behavior of the phase oscillators. The phases of the oscillators $\phi_{i, \mu}$ form a pseudo multicluster, see Definition 5.5.1. Further, the bounded modulations for the phases of each oscillator are given as Taylor expansions in $\epsilon$ with periodic coefficients that can be expressed as Fourier sums with even modes.

To determining the asymptotic expansion explicitly, derivatives of composed function have to be carried out. The following Lemma provides us with a general form.

Lemma A.4.1 Suppose we have n-times differentiable real functions $f$ and $g$. Let

$$
T_{n}:=\left\{\left(k_{1}, \ldots, k_{n}\right): 1 k_{1}+2 k_{2}+\cdots+n k_{n}=n, k_{1}, \ldots, k_{n} \in \mathbb{N}_{0}\right\}
$$


be written as

$$
D_{x}^{n}(f \circ g)\left(x_{0}\right)=\sum_{\left(k_{1}, \ldots, k_{n}\right) \in T_{n}} \frac{n !}{k_{1} ! \cdot \cdots \cdot k_{n} !}\left(D_{x}^{k_{1}+\ldots+k_{n}} f\right) \circ g\left(x_{0}\right) \prod_{m=1}^{n}\left(\frac{D^{m} g}{m !}\right)^{k_{m}}\left(x_{0}\right) .
$$

Proof. See [ABR88, pp. 95-96].

This expression for the $n$ th-derivative is also known as the Faà di Bruno formula.

Lemma A.4.2 Suppose the phase oscillators behave as

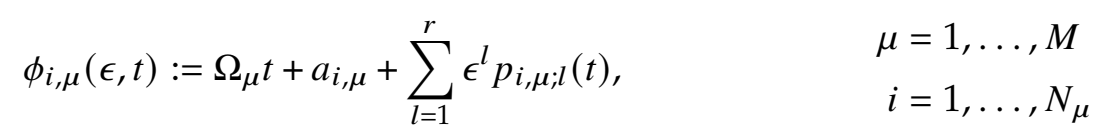

with $\Omega_{\mu} \in \mathbb{R}, a_{i, \mu} \in \mathbb{T}^{1}$ and there are $m_{i, \mu ; l} \in \mathbb{N}_{0}$ such that the bounded functions $p_{i, \mu ; l}: \mathbb{R} \rightarrow \mathbb{R}$ are given by the finite multi-Fourier series

$$
p_{i, \mu ; l}(t)=\sum_{\mathbf{m} \in \tilde{\mathbf{M}}\left(p_{i, \mu ;}\right)} c_{i, \mu ; l ; \mathbf{m}} e^{\mathrm{i} \Delta \Omega(\mathbf{m}) t}
$$

with $\left|\tilde{\mathbf{M}}\left(p_{i, \mu ; l}\right)\right|=m_{i, \mu ; l}$

Then, for $s \leq r$ there exist functions $k_{i j, \mu v ; l}(t)$ such that the asymptotic expansion of the pull-back attractor $\left(\kappa_{i j, \mu \nu}\right)_{*}$ defined in $(A .14)$ can be written as

$$
\left(\kappa_{i j, \mu \nu}\right)_{*}=\sum_{l=0}^{s} \epsilon^{l} k_{i j, \mu v ; l}(t)+\hat{R}_{i j, \mu \nu}(\epsilon, t) \quad \begin{aligned}
\mu, v & =1, \ldots, M \\
i & =1, \ldots, N_{\mu} \\
j & =1, \ldots, N_{v}
\end{aligned}
$$

where $\hat{R}_{i j, \mu \nu}(\epsilon, t) \in o\left(\epsilon^{s}\right)$ uniformly on $\mathbb{R}$ as $\epsilon \rightarrow 0$ and $\kappa_{i j, \mu \nu}^{(s)}(t):=\sum_{l=0}^{s} \epsilon^{l} k_{i j, \mu \nu ; l}(t)$. The $\kappa_{i j, \mu \nu}^{(s)}(t)$ is called the s-th-order asymptotic approximation of $\left(\kappa_{i j, \mu \nu}\right)_{*}$. Further, all $k_{i j, \mu \nu ; l}$ can also be written as a Fourier sum.

Proof. For fixed functions $\phi_{i, \mu}(t)$ the nonautonomous systems corresponding to the differential equation (2.26) possess the following parametrization of the pullback attractor

$$
\left(\kappa_{i j, \mu \nu}(t)\right)_{*}:=-\lim _{t_{0} \rightarrow-\infty}\left(\epsilon \int_{t_{0}}^{t} e^{-\epsilon\left(t-t^{\prime}\right)} \sin \left(\phi_{i, \mu}\left(t^{\prime}\right)-\phi_{j, v}\left(t^{\prime}\right)+\beta\right) \mathrm{d} t^{\prime}\right) .
$$

Using (A.15) with $f=\sin \left(\phi_{i, \mu}-\phi_{j, v}+\beta\right)$ and $g=\Delta \Omega_{\mu \nu} t+a_{i j, \mu \nu}+\beta+\sum_{l=1}^{r} \epsilon^{l} p_{i j, \mu \nu ; l}$ we can perform a Taylor expansion of $f(\epsilon, t)=\sin \left(\phi_{i, \mu}-\phi_{j, v}+\beta\right)$ around $\epsilon=0$. Due to Theorem 2.4.15 in [ABR88, pp. 93-94] we get

$$
\begin{aligned}
\sum_{l=0}^{s} \epsilon^{l} r_{i j, \mu \nu ; l}(\beta, t) & +\epsilon^{s} R_{i j, \mu v}(\epsilon, t):=\sin \left(\Delta \Omega_{\mu \nu} t+a_{i j, \mu \nu}+\beta+\sum_{l=1}^{r} \epsilon^{l} p_{i j, \mu v ; l}(t)\right) \\
= & \sin \left(\Delta \Omega_{\mu \nu} t+a_{i j, \mu \nu}+\beta\right)+\epsilon \cos \left(\Delta \Omega_{\mu \nu} t+a_{i j, \mu \nu}+\beta\right) p_{i j, \mu v ; 1}+\cdots+\epsilon^{s} R_{i j, \mu \nu}(\epsilon, t)
\end{aligned}
$$


where the abbreviations $p_{i j, \mu v ; l}:=p_{i, \mu ; l}-p_{j, v ; l}$ and $a_{i j, \mu v}:=a_{i, \mu}-a_{j, v}$ are used. Here, $R_{i j, \mu v}$ denotes the remainder of the Taylor expansion. The remainder $R(\epsilon, t) \rightarrow 0$ and $R_{i j, \mu v}(\epsilon, t) \in o(\epsilon)$ for all $t \in \mathbb{R}$ as $\epsilon \rightarrow 0$. We get

$$
\left(\kappa_{i j, \mu \nu}(t)\right)_{*}=-\sum_{l=0}^{s} \epsilon^{l+1} \int_{-\infty}^{t} e^{-\epsilon\left(t-t^{\prime}\right)} r_{i j, \mu \nu ; l}\left(\beta, t^{\prime}\right) \mathrm{d} t^{\prime}+\epsilon^{s+1} \int_{t_{0}}^{t} e^{-\epsilon\left(t-t^{\prime}\right)} R_{i j, \mu \nu}\left(\epsilon, t^{\prime}\right) \mathrm{d} t^{\prime} .
$$

In order to derive the expansion for $\left(\kappa_{i j, \mu v}\right)_{*}$ the integrals of the formula above have to be investigated. Faà di Bruno's formula (A.15) provides us with

$$
r_{i j, \mu \nu ; l}(\beta, t):=\sum_{\left(k_{1}, \ldots, k_{l}\right) \in T_{l}} \frac{\left(D^{k_{1}+\ldots+k_{l}} \sin \right)\left(\Delta \Omega_{\mu \nu} t+a_{i j, \mu \nu}+\beta\right)}{k_{1} ! \cdot \cdots \cdot k_{l} !} \prod_{m=1}^{l}\left(p_{i j, \mu v ; m}\right)^{k_{m}} .
$$

First, we conclude that the Taylor coefficients $r_{i j, \mu v ; l}(\beta)$ can also be written in a (finite) multiFourier sum

$$
r_{i j, \mu v ; l}(\beta, t)=\sum_{\mathbf{m} \in \tilde{\mathbf{M}}\left(r_{i j, \mu v ; l}(\beta)\right)} d_{i j, \mu v ; l ; \mathbf{m}}(\beta) e^{\mathrm{i} \Delta \Omega(\mathbf{m}) t}
$$

Second, $d_{i j, \mu \nu ; l ; 0} \neq 0$ if $\prod_{m=1}^{l}\left(p_{i j, \mu \nu, m}\right)^{k_{m}}$ possess a non vanishing term for $e^{\mathrm{i} \Delta \Omega_{\mu \nu} t}$. With this, we are able to calculate the integrals in (A.17) and get

$$
\begin{aligned}
\int_{-\infty}^{t} e^{-\epsilon\left(t-t^{\prime}\right)} r_{i j, \mu v ; l}(\beta, t) & =\sum_{\mathbf{m} \in \tilde{\mathbf{M}}\left(r_{i j, \mu \nu ; l}(\beta)\right)} d_{i j, \mu v ; l ; \mathbf{m}}(\beta) \int_{-\infty}^{t} e^{-\epsilon\left(t-t^{\prime}\right)} e^{\mathrm{i} \Delta \Omega(\mathbf{m}) t^{\prime}} \mathrm{d} t^{\prime} \\
& =\sum_{\mathbf{m} \in \tilde{\mathbf{M}}\left(r_{i j, \mu v ; l}(\beta)\right)}\left(\frac{1}{\epsilon+\mathrm{i} \Delta \Omega(\mathbf{m})}\right) d_{i j, \mu v ; l ; \mathbf{m}}(\beta) e^{\mathrm{i} \Delta \Omega(\mathbf{m}) t}
\end{aligned}
$$

The last term in the equation (A.17) is in $o\left(\epsilon^{s}\right)$ which can be seen as follows. Since $R_{i j, \mu \nu}(\epsilon, t) \epsilon$ $o(\epsilon)$ as $\epsilon \rightarrow 0$ for all $C>0$ there exist an $\epsilon_{0}(t)>0$ such that $\left|R_{i j, \mu \nu}(\epsilon, t)\right|<C \epsilon$ for all $\epsilon<\epsilon_{0}(t)$. Due to the boundedness of all $p_{i, \mu ; l}$ the remainder $R_{i j, \mu \nu}(\epsilon, t)$ is also bounded by some positive number $\tilde{C}$. Thus, $R_{i j, \mu \nu}(\epsilon, t) \in o(\epsilon)$ uniformly on $\mathbb{R}$ as $\epsilon \rightarrow 0$, hence for all $C>0$

$$
\left|\int_{t_{0}}^{t} e^{-\epsilon\left(t-t^{\prime}\right)} R\left(\epsilon, t^{\prime}\right) \mathrm{d} t^{\prime}\right| \leq C\left|1-e^{\epsilon\left(t_{0}-t\right)}\right| .
$$

Finally, we end up with

$$
\left(\kappa_{i j, \mu \nu}\right)_{*}(t)=-\sum_{l=0}^{s} \epsilon^{l} \sum_{\mathbf{m} \in \tilde{\mathbf{M}}\left(r_{i j, \mu v ; l}^{\beta}\right)} d_{i j, \mu v ; l ; \mathbf{m}}^{\beta} \frac{1}{1+\mathrm{i} \frac{\Delta \Omega(\mathbf{m})}{\epsilon}} e^{\mathrm{i} \Delta \Omega(\mathbf{m}) t}+\tilde{R}_{i j, \mu \nu}(\epsilon, t)
$$

where $\tilde{R}_{i j, \mu v}:=\left(\epsilon^{s+1} \int_{t_{0}}^{t} e^{-\epsilon\left(t-t^{\prime}\right)} R\left(\epsilon, t^{\prime}\right) \mathrm{d} t^{\prime}\right)_{*} \in o\left(\epsilon^{s}\right)$ uniformly on $\mathbb{R}$ as $\epsilon \rightarrow 0$. By considering the Laurent series

$$
\frac{1}{1+\mathrm{i} \frac{\Delta \Omega(\mathbf{m})}{\epsilon}}=-\sum_{n=1}^{\infty} i^{n}\left(\frac{\epsilon}{\Delta \Omega(\mathbf{m})}\right)^{n}
$$


which converges whenever $\epsilon<|\Delta \Omega(\mathbf{m})|$, the coefficients of the expansion $\left(\kappa_{i j, \mu \nu}\right)_{*}^{(s)}=$ $\sum_{l=0}^{s} \epsilon^{l} k_{i j, \mu v ; l}(t)$ are given by

$$
\begin{aligned}
\kappa_{i j, \mu \nu ; 0} & =-d_{i j, \mu v ; 0 ; 0}(\beta), \\
\kappa_{i j, \mu v ; l>0} & =-d_{i j, \mu v ; l ; 0}(\beta)+\sum_{n=0}^{l-1} \sum_{\mathbf{m} \in \tilde{\mathbf{M}}\left(r_{i j, \mu v ; n}(\beta)\right) /\{0\}} i^{l-n} \frac{d_{i j, \mu v ; n ; \mathbf{m}}(\beta)}{(\Delta \Omega(\mathbf{m}))^{l-n}} e^{\mathrm{i} \Delta \Omega(\mathbf{m}) t} .
\end{aligned}
$$

Note that, $\epsilon$ can always be chosen such that $\epsilon<|\Delta \Omega(\mathbf{m})|$, since we consider the asymptotic limit $\epsilon \rightarrow 0$. The coefficients are determined via comparing the terms of both sides of the equation (A.20) with respect to their order in $\epsilon$. In the case $\mu \neq v$ we get $\kappa_{i j, \mu v ; 0}=0$. All terms of order $O\left(\epsilon^{s+1}\right)$ and $\tilde{R}_{i j, \mu \nu}(\epsilon, t)$ are summarized in $\hat{R}_{i j, \mu v}(\epsilon, t) \in o\left(\epsilon^{s}\right)$ as $\epsilon \rightarrow 0$.

Remark A.4.2 Without considering the asymptotic limit $\epsilon \rightarrow 0$, the $s$-th order formal expansion for $\kappa_{i j, \mu \nu}$ would have the form as it is given in Lemma A.4.2 under the condition that $\Delta \Omega(\mathbf{m})>\epsilon$ for all $\mathbf{m} \in \bigcup_{l=1}^{s-1} \tilde{\mathbf{M}}\left(r_{i j, \mu \nu ; l}^{\beta}\right)$.

Lemma A.4.3 Suppose everything is given as in Lemma A.4.2. Then, if for all $\mu \in 1, \ldots, M, i \in$ $\left\{1, \ldots, N_{\mu}\right\}$ and $l \in 1, \ldots, r, p_{i, \mu ; l}(t)$ can be written completely in terms of even modes, i.e., all $m_{1}, \ldots, m_{M}$ are even for $\left(m_{1}, \ldots, m_{M}\right) \in \tilde{\boldsymbol{M}}\left(p_{i, \mu ;}\right)$, then for all $\left(n_{1}, \ldots, n_{M}\right) \in \tilde{\boldsymbol{M}}\left(\kappa_{i j, \mu v}^{S}(t)\right)$ holds: $n_{\lambda}$ are even for $\lambda \neq \mu, v$ and odd otherwise.

Proof. It is fairly easy to verify that if $p_{i, \mu ; l}(t)$ can be completely written in terms of even modes for all $i, \mu, l$, so can $p_{i j, \mu v ; l}(t)$ and moreover, the product $p_{i j, \mu v ; l} \cdot p_{i j, \mu v ; m}$. According to (A.18) $r_{i j, \mu v ; l}$ consists only of terms of the form $e^{ \pm \mathrm{i} \Delta \Omega_{\mu \nu} t} \cdot e^{\mathrm{i} \Delta \Omega(m) t}$ and hence $m_{\lambda}$ are even for every $\lambda \neq \mu, v$ and odd otherwise for all $\left(m_{1}, \ldots, m_{M}\right) \in \tilde{\mathbf{M}}\left(r_{i j, \mu v ; l}\right)$. Since integration by time (A.17) does not make any changes in the modes, the same holds for $k_{i j, \mu v ; l}(t)$ and hence $\kappa_{i j, \mu v}^{s}(t)$.

Now, we have everything which is needed to proof the main result in Proposition 5.3.1.

Proof. Note, whenever we write $\Delta \Omega(\mathbf{m}), \Delta \Omega^{(r)}(\mathbf{m})$ is meant. We omit the superscript for the sake of readability. (i) Combing the formal time derivative of the first equation in (5.11), the system equations (2.25)-(2.26) and Lemma A.4.2 we get

$$
\dot{\phi}_{i, \mu}^{(r)}=\sum_{l=0}^{r} \epsilon^{l} \frac{\partial \Omega_{\mu}^{(r)}}{\partial \tau_{l}}+\sum_{l=1}^{r} \epsilon^{l} \dot{p}_{i, \mu ; l}(t)=-\frac{1}{N} \sum_{\nu=1}^{M} \sum_{j=1}^{N_{v}} \sum_{l=0}^{r} \sum_{n=0}^{r} \epsilon^{l+n} k_{i j, \mu \nu ; l}(t) r_{i j, \mu v, n}(\alpha, t) .
$$

Assume that $p_{i, \mu ; l}(t)=\sum_{\mathbf{m} \in \tilde{\mathbf{M}}\left(p_{i, \mu ; l}\right)} c_{i, \mu ; l ; \mathbf{m}} e^{\mathrm{i} \Delta \Omega(\mathbf{m}) t}$ with $\left|\tilde{\mathbf{M}}\left(p_{i, \mu ; l}\right)\right| \in \mathbb{N}$. We get

$$
\begin{aligned}
\frac{\partial \Omega_{\mu}^{(r)}}{\partial t} & =\frac{1}{N} \sum_{v=1}^{M} \sum_{j=1}^{N_{v}} \sum_{\mathbf{m} \in \tilde{\mathbf{M}}\left(r_{i j, \mu v ; 0}(\alpha)\right)} d_{i j, \mu v ; 0 ; \mathbf{m}}(\alpha) d_{i j, \mu v ; 0 ; 0}(\beta) e^{\mathrm{i} \Delta \Omega(\mathbf{m}) t}, \\
\frac{\partial \Omega_{\mu}^{(r)}}{\partial \tau_{l}}+\dot{p}_{i, \mu ; l}(t) & =-\frac{1}{N} \sum_{v=1}^{M} \sum_{j=1}^{N_{v}} \sum_{m=0}^{l} k_{i j, \mu v ; m} r_{i j, \mu v, l-m}(\alpha)
\end{aligned}
$$


by comparing both sides of the equation (A.21) with respect to the order of $\epsilon$. Due to Lemma A.4.2

$$
\frac{\partial \Omega_{\mu}^{(r)}\left(\tau_{0}, \ldots, \tau_{m}\right)}{\partial t}=\frac{1}{N} \sum_{j=1}^{N_{\mu}} d_{i j, \mu \mu ; 0 ; 0}(\alpha) d_{i j, \mu \mu ; 0 ; 0}(\beta)=: \Omega_{\mu, 0} \in \mathbb{R} .
$$

This equation can be solved by $\tilde{\Omega}_{\mu}=\Omega_{\mu, 0} t+\tilde{\Omega}_{\mu, 0}\left(\tau_{1}, \ldots, \tau_{m}\right)$. Due to our assumptions the right hand side of equation (A.22) can be written as

$$
\begin{aligned}
&-\sum_{v=1}^{M} \sum_{j=1}^{N_{\nu}} \sum_{m=0}^{l} k_{i j, \mu v ; m} r_{i j, \mu v, l-m}(\alpha) \\
& \sum_{\nu=1}^{M} \sum_{j=1}^{N_{v}} \sum_{m=0}^{l}\left[d_{i j, \mu v ; m ; 0}(\beta)-\sum_{n=0}^{m-1} \sum_{\mathbf{m} \in \tilde{\mathbf{M}}\left(r_{i j, \mu v ; n}(\beta)\right) /\{0\}} i^{m-n} \frac{d_{i j, \mu v ; n ; \mathbf{m}}(\beta)}{(\Delta \Omega(\mathbf{m}))^{m-n}} e^{\mathrm{i} \Delta \Omega(\mathbf{m}) t}\right] \times \\
& {\left[d_{i j, \mu v ; l-m ; \mathbf{0}}(\alpha)+\sum_{\mathbf{m} \in \tilde{\mathbf{M}}\left(r_{i j, \mu v ; l-m}(\alpha)\right) /\{0\}} d_{i j, \mu v ; l-m ; \mathbf{m}}(\alpha) e^{\mathrm{i} \Delta \Omega(\mathbf{m}) t}\right] . }
\end{aligned}
$$

By using 1.) and 2.) of Lemma A.4.2 we find

$$
\begin{aligned}
& \frac{\partial \Omega_{\mu}^{(r)}}{\partial \tau_{l}}=\frac{1}{N} \sum_{v=1}^{M} \sum_{j=1}^{N_{v}} \sum_{m=0}^{l}\left[d_{i j, \mu v ; m ; 0}(\beta) d_{i j, \mu v ; l-m ; 0}(\alpha)\right. \\
& \left.-\sum_{n=0}^{m-1} \sum_{\mathbf{m} \in \tilde{\mathbf{M}}\left(r_{i j, \mu v ; n}(\beta)\right) /\{0\}} i^{m-n} \frac{d_{i j, \mu v ; n ; \mathbf{m}}(\beta) d_{i j, \mu \nu ; l-m ;-\mathbf{m}}(\alpha)}{(\Delta \Omega(\mathbf{m}))^{m-n}}\right], \\
& \dot{p}_{i, \mu ; l}=\frac{1}{N} \sum_{\nu=1}^{M} \sum_{j=1}^{N_{v}} \sum_{m=0}^{l}\left[d_{i j, \mu v ; m ; 0}(\beta)\left(\sum_{\mathbf{m} \in \tilde{\mathbf{M}}\left(r_{i j, \mu v ; l-m}(\beta)\right) /\{0\}} d_{i j, \mu v ; l-m ; \mathbf{m}}(\alpha) e^{\mathrm{i} \Delta \Omega(\mathbf{m}) t}\right)\right. \\
& -d_{i j, \mu v ; l-m ; 0}(\alpha)\left(\sum_{n=0}^{m-1} \sum_{\mathbf{m} \in \tilde{\mathbf{M}}\left(r_{i j, \mu v ; n}(\beta)\right) /\{0\}} i^{m-n} \frac{d_{i j, \mu v ; n ; \mathbf{m}}(\beta)}{(\Delta \Omega(\mathbf{m}))^{m-n}} e^{\mathrm{i} \Delta \Omega(\mathbf{m}) t}\right) \\
& \left.-\sum_{n=0}^{m-1} \sum_{\substack{\mathbf{m} \in \tilde{\mathbf{M}}\left(r_{i j, \mu \nu ; n}(\beta)\right) /\{0\} \\
\mathbf{n} \in \tilde{\mathbf{M}}\left(r_{i j, \mu \nu ; l-m}(\alpha)\right) /\{0,-\mathbf{m}\}}} i^{m-n} \frac{d_{i j, \mu v ; n ; \mathbf{m}}(\beta) d_{i j, \mu \nu ; l-m ; \mathbf{n}}(\alpha)}{(\Delta \Omega(\mathbf{m}))^{m-n}} e^{\mathrm{i} \Delta \Omega(\mathbf{m}+\mathbf{n}) t}\right] .
\end{aligned}
$$

Note that we use the multi-time scale function $\Omega_{\mu}^{(r)}$ to deal with all terms of the expansion describing a linear growth. All the other terms are considered to determine the behaviour of $p_{i, \mu ; l}$. With this ansatz we are able to maintain the boundedness of $p_{i, \mu ; l}$ while letting $\Omega_{\mu}^{(r)}$ alone describing unbounded behaviour in $t \in \mathbb{R}$. Note further that $\Omega_{\mu}^{(r)}$ can be directly computed if all functions $p_{i, \mu ; k \leq l}(t)$ are known. Thus, we finally end up with

$$
\Omega_{\mu}^{(r)}=\sum_{l=0}^{r} \epsilon^{l} \Omega_{\mu, l} t
$$


We assume now that for all $i, \mu$ and $l>1, p_{i, \mu ; l}$ can be written completely in terms of even modes, c.f., Lemma A.4.3. In particular, $(\mu \nu) \notin M\left(p_{i, \mu ; l}\right)$. Thus, $d_{i j, \mu v ; l ; 0}(\beta)=0$ by (A.18) for all $\mu, v=1, \ldots, M, i=1, \ldots, N_{\mu}, j=1, \ldots, N_{\nu}$ and $l \geq 1$ and

$$
\dot{p}_{i, \mu ; l}=-\frac{1}{N} \sum_{\nu=1}^{M} \sum_{j=1}^{N_{\nu}} \sum_{\substack{m=1 \\ n=0}}^{l, m-1} \sum_{\substack{\mathbf{m} \in \tilde{\mathbf{M}}\left(r_{i j, \mu \nu ; n}(\beta)\right) /\{0\} \\ \mathbf{n} \in \tilde{\mathbf{M}}\left(r_{i j, \mu \nu ; l-m}(\alpha)\right) /\{0,-\mathbf{m}\}}} i^{m-n} \frac{d_{i j, \mu \nu ; n ; \mathbf{m}}(\beta) d_{i j, \mu \nu ; l-m ; \mathbf{n}}(\alpha)}{(\Delta \Omega(\mathbf{m}))^{m-n}} e^{\mathrm{i} \Delta \Omega(\mathbf{m}+\mathbf{n}) t} .
$$

Hence, we get an equation to determine the value of $p_{i, \mu ; l}$ inductively. Due to Lemma A.4.3, we know that if all $p_{i, \mu ; l}$ can be written in terms of even modes then $m_{\mu}$ and $m_{\nu}$ are odd for all $\left(m_{1}, \ldots, m_{M}\right) \in \tilde{\mathbf{M}}\left(r_{i j, \mu v ; l}(\alpha)\right)$. Therefore $p_{i, \mu ; l}$ can be written in terms of even modes. This is consistent with our assumption that $p_{i, \mu ; l}$ can be written in terms of even modes. Consider further

$$
\dot{p}_{i, \mu ; 1}=-\frac{1}{N} \sum_{v=1}^{M} \sum_{j=1}^{N_{v}} \sum_{\substack{\mathbf{m} \in \tilde{\mathbf{M}}\left(r_{i j, \mu v ; 0}(\beta)\right) /\{0\} \\ \mathbf{n} \in \tilde{\mathbf{M}}\left(r_{i j, \mu v ; 0}(\alpha)\right) /\{0,-\mathbf{m}\}}} i \frac{d_{i j, \mu v ; 0 ; \mathbf{m}}(\beta) d_{i j, \mu v ; 0 ; \mathbf{n}}(\alpha)}{(\Delta \Omega(\mathbf{m}))^{m-n}} e^{\mathrm{i} \Delta \Omega(\mathbf{m}+\mathbf{n}) t}
$$

The expression for $p_{i, \mu, 1}$ can be found by integration

$$
p_{i, \mu ; 1}(t)=-\frac{1}{N} \sum_{\nu=1}^{M} \sum_{j=1}^{N_{v}} \sum_{\substack{\mathbf{m} \in \tilde{\mathbf{M}}\left(r_{i j, \mu v ; 0}(\beta)\right) /\{0\} \\ \mathbf{n} \in \mathbf{M}\left(r_{i j, \mu v ; 0}(\alpha)\right) /\{0,-\mathbf{m}\}}} \frac{d_{i j, \mu v ; 0 ; \mathbf{m}}(\beta) d_{i j, \mu v ; 0 ; \mathbf{n}}(\alpha)}{(\Delta \Omega(\mathbf{m})) \Delta \Omega(\mathbf{m}+\mathbf{n})} e^{\mathrm{i} \Delta \Omega(\mathbf{m}+\mathbf{n}) t} .
$$

Since $p_{i, \mu ; 1}$ can be written as a Fourier sum the same holds true for all $p_{i, \mu, l}$ by induction. This is consistent with our assumption for $p_{i, \mu, l}$ in the beginning of this proof. The expressions $k_{i j, \mu v ; l}$ follow from Lemma A.4.2. Furthermore, analog to Lemma A.4.2, from Theorem 2.4.15 in [ABR88, pp. 93-94] we conclude $\kappa_{i j, \mu \nu}(\epsilon, t)-\kappa_{i j, \mu \nu}^{r}(\epsilon, t) \in O\left(\epsilon^{r}\right)$ and $\phi_{i, \mu}(\epsilon, t)-\phi_{i, \mu}^{(r)}(\epsilon, t) \in O\left(\epsilon^{r}\right)$ for $t \in O\left(1 / \epsilon^{r}\right)$ as $\epsilon \rightarrow 0$.

(ii) To achieve this result we apply now (i) which allows for iteratively determining the function appearing in the asymptotic expansion.

0 -th order: For the expansion of the sine-function from equation (A.18) we find $r_{i j, \mu \mu ; 0}(\beta)=$ $\sin \left(a_{i j, \mu \mu}+\beta\right)=d_{i j, \mu \mu ; 0 ; 0}(\beta)$ and

$$
r_{i j, \mu v ; 0}(\beta)=\sin \left(\Delta \Omega_{\mu \nu}^{(1)} t+a_{i j, \mu \nu}+\beta\right)=d_{i j, \mu v ; 0 ;(\mu \nu)}(\beta) e^{\mathrm{i} \Delta \Omega_{\mu \nu}^{(1)} t}+c . c .
$$

where $d_{i j, \mu v ; 0 ;(\mu \nu)}(\beta):=(1 / 2 \mathrm{i}) e^{\mathrm{i}\left(a_{i j, \mu \nu}+\beta\right)}$ and c.c. stands for complex conjugated. Hence, for the coupling matrix we find

$$
\kappa_{i j, \mu \mu ; 0}=-\sin \left(a_{i j, \mu \mu}+\beta\right)
$$

Depending on the cluster the zero-th order approximation for the frequencies read

$$
\Omega_{\mu, 0}=\frac{1}{N} \sum_{j=1}^{N_{\mu}} \sin \left(a_{i j, \mu \mu}+\beta\right) \sin \left(a_{i j, \mu \mu}+\beta\right)=\frac{n_{\mu}}{2}(\cos (\alpha-\beta)-\cos (\alpha+\beta))
$$


for all $\mu=1, \ldots, M_{1}$ and analogously $\Omega_{\mu, 0}=\frac{n_{\mu}}{2} \cos (\alpha-\beta)$ for all $\mu=M_{1}+1, \ldots, M$.

1-th order: Since we know the 0-th order expansion we are able to calculate the next order. We get

$$
\begin{aligned}
\Omega_{\mu, 1} & =-\frac{1}{N} \sum_{\substack{\nu=1 \\
\nu \neq \mu}}^{M_{1}} \sum_{j=1}^{N_{\nu}} \sum_{\mathbf{m} \in\{(\mu \nu),-(\mu \nu)\}} i \frac{d_{i j, \mu \nu ; 0 ; \mathbf{m}}(\beta) d_{i j, \mu \nu ; 0 ;-\mathbf{m}}(\alpha)}{\Delta \Omega^{(1)}(\mathbf{m})} \\
& =\frac{1}{2 N} \sum_{\substack{\nu=1 \\
\nu \neq \mu}}^{M} \sum_{j=1}^{N_{\nu}} \frac{1}{2 \mathrm{i}}\left(\frac{e^{\mathrm{i}\left(a_{i j, \mu \nu}+\beta\right)} e^{-\mathrm{i}\left(a_{i j, \mu \nu}+\alpha\right)}}{\Delta \Omega_{\mu \nu}^{(1)}}-\frac{e^{-\mathrm{i}\left(a_{i j, \mu \nu}+\beta\right)} e^{\mathrm{i}\left(a_{i j, \mu \nu}+\alpha\right)}}{\Delta \Omega_{\mu \nu}^{(1)}}\right) \\
& =\frac{1}{2 N} \sum_{\substack{\nu=1 \\
\nu \neq \mu}}^{M} \sum_{j=1}^{N_{\nu}} \frac{1}{2 \mathrm{i}}\left(\frac{e^{\mathrm{i}(\beta-\alpha)}}{\Delta \Omega_{\mu \nu}^{(1)}}-\frac{e^{-\mathrm{i}(\beta-\alpha)}}{\Delta \Omega_{\mu \nu}^{(1)}}\right)=-\sum_{\substack{\nu=1 \\
\nu \neq \mu}}^{M} \frac{n_{\nu}}{2 \Delta \Omega_{\mu \nu}^{(1)}} \sin (\alpha-\beta) .
\end{aligned}
$$

For all $\mu=1, \ldots, M_{1}$ we get

$$
\begin{aligned}
\dot{p}_{\mu ; 1}= & -\frac{1}{N} \sum_{\substack{\nu=1 \\
\nu \neq \mu}}^{M_{1}} \sum_{j=1}^{N_{\nu}} \sum_{\mathbf{m} \in\{(\mu \nu),-(\mu \nu)\}}\left(i \frac{d_{i j, \mu v ; 0 ; \mathbf{m}}(\beta) d_{i j, \mu v ; 0 ; \mathbf{m}}(\alpha)}{\Delta \Omega^{(1)}(\mathbf{m})}\right) e^{\mathrm{i} 2 \Delta \Omega^{(1)}(\mathbf{m}) t} \\
& +\sum_{\nu=M_{1}+1}^{M} \sum_{j=1}^{N_{\nu}} \sum_{\mathbf{m} \in\{(\mu \nu),-(\mu \nu)\}}\left(i \frac{d_{i j, \mu \nu ; 0 ; \mathbf{m}}(\beta) d_{i j, \mu \nu ; 0 ; \mathbf{m}}(\alpha)}{\Delta \Omega^{(1)}(\mathbf{m})}\right) e^{\mathrm{i} 2 \Delta \Omega^{(1)}(\mathbf{m}) t} \\
= & -\frac{1}{2} \sum_{\substack{\nu=1 \\
\nu \neq \mu}}^{M} \frac{n_{\nu}}{2 \mathrm{i}}\left(\frac{e^{\mathrm{i}\left(a_{\mu}-a_{\nu}+\alpha+\beta\right)}}{\Delta \Omega_{\mu \nu}^{(1)}}\right) e^{\mathrm{i} 2 \Delta \Omega_{\mu \nu}^{(1)} t}-\frac{n_{\nu}}{2 \mathrm{i}}\left(\frac{e^{-\mathrm{i}\left(a_{\mu}-a_{\nu}+\alpha+\beta\right)}}{\Delta \Omega_{\mu \nu}^{(1)}}\right) e^{-\mathrm{i} 2 \Delta \Omega_{\mu \nu}^{(1)} t}
\end{aligned}
$$

and for all $\mu=M_{1}+1, \ldots, M$ we get

$$
\begin{aligned}
& \dot{p}_{i, \mu ; 1}=-\frac{1}{N} \sum_{\nu=1}^{M_{1}} \sum_{j=1}^{N_{v}} \sum_{\mathbf{m} \in\{(\mu v),-(\mu \nu)\}}\left(i \frac{d_{i j, \mu v ; 0 ; \mathbf{m}}(\beta) d_{i j, \mu v ; 0 ; \mathbf{m}}(\alpha)}{\Delta \Omega^{(1)}(\mathbf{m})}\right) e^{\mathrm{i} 2 \Delta \Omega^{(1)}(\mathbf{m}) t} \\
& +\sum_{\substack{\nu=M_{1}+1 \\
v \neq \mu}}^{M} \sum_{j=1}^{N_{v}} \sum_{\mathbf{m} \in\{(\mu \nu),-(\mu \nu)\}}\left(i \frac{d_{i j, \mu v ; 0 ; \mathbf{m}}(\beta) d_{i j, \mu v ; 0 ; \mathbf{m}}(\alpha)}{\Delta \Omega^{(1)}(\mathbf{m})}\right) e^{\mathrm{i} 2 \Delta \Omega^{(1)}(\mathbf{m}) t} \\
& =-\frac{1}{2} \sum_{\nu=1}^{M} \frac{n_{\nu}}{2 \mathrm{i}}\left(\frac{e^{\mathrm{i}\left(a_{i, \mu}-a_{\nu}+\alpha+\beta\right)}}{\Delta \Omega_{\mu \nu}^{(1)}}\right) e^{\mathrm{i} 2 \Delta \Omega_{\mu \nu}^{(1)} t}-\frac{n_{\nu}}{2 \mathrm{i}}\left(\frac{e^{-\mathrm{i}\left(a_{i, \mu}-a_{\nu}+\alpha+\beta\right)}}{\Delta \Omega_{\mu \nu}^{(1)}}\right) e^{-\mathrm{i} 2 \Delta \Omega_{\mu \nu}^{(1)} t} .
\end{aligned}
$$

Thus, solving this fairly easy differential equation the following expression is obtained for $\mu=1, \ldots, M_{1}$

$$
p_{\mu ; 1}=\frac{1}{4} \sum_{\substack{\nu=1 \\ \nu \neq \mu}}^{M} \frac{n_{\nu}}{2}\left(\frac{e^{\mathrm{i}\left(a_{\mu}-a_{\nu}+\alpha+\beta\right)}}{\left(\Delta \Omega_{\mu \nu}^{(1)}\right)^{2}}\right) e^{\mathrm{i} 2 \Delta \Omega_{\mu \nu}^{(1)} t}+\frac{n_{\nu}}{2}\left(\frac{e^{-\mathrm{i}\left(a_{\mu}-a_{\nu}+\alpha+\beta\right)}}{\left(\Delta \Omega_{\mu \nu}^{(1)}\right)^{2}}\right) e^{-\mathrm{i} 2 \Delta \Omega_{\mu \nu}^{(1)} t} .
$$

Analogously we find the expression for $p_{i, \mu ; 1}$ with $\mu=M_{1}+1, \ldots, M$. For the coupling matrix 
we get

$$
\begin{aligned}
\kappa_{i j, \mu \nu ; 1} & =\sum_{\mathbf{m} \in\{(\mu \nu),-(\mu \nu)\}}\left(i \frac{d_{i j, \mu \nu ; 0 ; \mathbf{m}}(\beta)}{\Delta \Omega^{(1)}(\mathbf{m})}\right) e^{\mathrm{i} \Delta \Omega^{(1)}(\mathbf{m}) t} \\
& =\frac{e^{\mathrm{i}\left(a_{i j, \mu \nu}+\beta\right)}}{2 \Delta \Omega_{\mu \nu}^{(1)}} e^{\mathrm{i} \Delta \Omega_{\mu \nu}^{(1)} t}+\frac{e^{-\mathrm{i}\left(a_{i j, \mu \nu}+\beta\right)}}{2 \Delta \Omega_{\mu \nu}^{(1)}} e^{-\mathrm{i} \Delta \Omega_{\mu \nu}^{(1)} t} .
\end{aligned}
$$

\section{A.5 From local to global order parameter}

From expression (6.9) we derived two types of one-cluster solution namely antipodal or local splay states. In the following we derive a remarkable relation between local and global properties on a nonlocal ring which is: If a cluster is of local splay type, the cluster is also of global splay type.

In order to show this, we rewrite the sum $\sum_{i=1}^{N} Z_{i}^{(2)}$ in two ways. Firstly, using the pure definition of the local order parameter (6.4):

$$
\sum_{i=1}^{N} Z_{i}^{(2)}(\chi)=\frac{1}{2 P} \sum_{i, j=1}^{N} a_{i j} e^{\mathrm{i} 2 \chi_{j}}=\sum_{j=1}^{N} e^{\mathrm{i} 2 \chi_{j}}=N Z^{(2)}(\chi)
$$

Secondly, the sum can be rewritten using the definition of a local splay type cluster with $R_{i}^{(2)}(\chi)=R_{c}^{(2)}(\chi)$ and $2 \chi_{i}=\vartheta_{i}^{(2)}$. Then

$$
\sum_{i=1}^{N} Z_{i}^{(2)}(\chi)=\sum_{i=1}^{N} R_{c}^{(2)} e^{\mathrm{i} \vartheta_{i}^{(2)}}=R_{c}^{(2)}(\chi) \sum_{i=1}^{N} e^{\mathrm{i} 2 \chi_{i}}=N R_{c}^{(2)}(\chi) Z^{(2)}(\chi)
$$

By equating (A.23) and (A.24) we obtain:

$$
\left(1-R_{c}^{(2)}(\chi)\right) Z^{(2)}(\chi)=0
$$

The latter equation yields $R^{(2)}(\chi)=0$ for all local splay type clusters, since $R_{c}^{(2)}(\chi)<1$ by definition. 


\section{A.6 Stability of one-cluster states on nonlocally coupled networks}

First note that the set of equations (6.15)-(6.16) can be brought into the following block form

$$
\frac{\mathrm{d}}{\mathrm{d} t}\left(\begin{array}{c}
\delta \phi \\
\delta \kappa
\end{array}\right)=\left(\begin{array}{cc}
M & B \\
C & -\epsilon \mathbb{I}_{N^{2}}
\end{array}\right)\left(\begin{array}{c}
\delta \phi \\
\delta \kappa
\end{array}\right)
$$

where $(\delta \boldsymbol{\phi})^{T}=\left(\delta \phi_{1}, \ldots, \delta \phi_{N}\right),(\delta \kappa)^{T}=\left(\delta \kappa_{11}, \ldots, \delta \kappa_{1 N}, \delta \kappa_{21}, \ldots, \delta \kappa_{N N}\right), \quad B=\left(\begin{array}{lll}B_{1} & \cdots & B_{N}\end{array}\right)$, $C=\left(\begin{array}{c}C_{1} \\ \vdots \\ C_{N}\end{array}\right)$, and $M, B_{n}, C_{n}$ are $N \times N$ matrices with $n=1, \ldots, N$. The elements of the block matrices read

$$
\begin{aligned}
m_{i j} & = \begin{cases}-\frac{\sigma}{2} \sin (\alpha-\beta) \sum_{k=1}^{N} a_{i k}-\sigma a_{i i} \sin (\beta) \cos (\alpha)+\frac{\sigma}{2} \sum_{k=1}^{N} a_{i k} \sin \left(2\left(a_{i}-a_{k}\right)+\alpha+\beta\right), & i=j \\
\frac{\sigma a_{i j}}{2}\left(\sin (\alpha-\beta)-\sin \left(2\left(a_{i}-a_{j}\right)+\alpha+\beta\right)\right), & i \neq j\end{cases} \\
& = \begin{cases}-\frac{\sigma}{2}\left(\sin (\alpha-\beta)+\mathfrak{J}\left(e^{-\mathrm{i}\left(2 a_{i}+\alpha+\beta\right)} Z_{i}^{(2)}(\boldsymbol{a})\right)\right) \sum_{k=1}^{N} a_{i k}-\sigma a_{i i} \sin (\beta) \cos (\alpha) & i=j \\
\frac{\sigma a_{i j}}{2}\left(\sin (\alpha-\beta)-\sin \left(2\left(a_{i}-a_{j}\right)+\alpha+\beta\right)\right), & i \neq j\end{cases} \\
b_{i j ; n} & = \begin{cases}-\sigma a_{n j} \sin \left(a_{n}-a_{j}+\alpha\right), & i=n \\
0, & \text { otherwise }\end{cases} \\
c_{i j ; n} & = \begin{cases}0, & j=n, i=j \\
-\epsilon a_{n i} \cos \left(a_{n}-a_{i}+\beta\right), & j=n, i \neq j \\
\epsilon a_{n i} \cos \left(a_{n}-a_{i}+\beta\right), & j \neq n, i=j \\
0, & \text { otherwise }\end{cases}
\end{aligned}
$$

where $\sigma=1 / 2 P$. Note that we use the Schur decomposition (4.11) throughout this stability analysis. Suppose we have a phase locked state with phases $a_{i} \in[0,2 \pi)$. Then, the solution for the characteristic equations corresponding to the linearized system (6.15)-(6.16) are given by $\lambda=-\epsilon$ with multiplicity $N^{2}-N$ and by the solution of the following set of equations, see also Lemma A.2.2,

$$
\operatorname{det}\left(\left(M-\lambda \mathbb{I}_{N}\right)(\epsilon+\lambda)+B C\right)=0 \text {. }
$$

The second term $D:=B C$ of the Schur complement are element wise given by

$$
d_{i j}=-\frac{\epsilon \sigma}{2} a_{i j}\left(\sin (\alpha-\beta)+\sin \left(2\left(a_{i}-a_{j}\right)+\alpha+\beta\right)\right)
$$

if $i \neq j$ and

$$
d_{i i}=\frac{\epsilon \sigma}{2}\left(\sin (\alpha-\beta)-\mathfrak{J}\left(e^{-\mathrm{i}\left(2 a_{i}+\alpha+\beta\right)} Z_{i}^{(2 k)}(\boldsymbol{a})\right)\right) \sum_{k=1}^{N} a_{i k}-\epsilon \sigma \sin (\alpha) \cos (\beta)
$$


Consider that $a_{i}(k)=i k \frac{2 \pi}{N}$ and the base topology has constant row sum $\rho \in \mathbb{N}$, i.e. $\sum_{k=1}^{N} a_{i k}=\rho$, then the matrix in (A.25) becomes circulant. For a ring structure, as considered in this thesis, we have $\rho=2 P$. Hence it can be diagonalized using the $N$ eigenvectors $\zeta_{l}=\exp (\mathrm{i} 2 \pi l / N)=$ $\exp \left(\mathrm{i} a_{l}(1)\right)$ and the eigenvalues $\mu_{l}(\lambda)$ are

$$
\begin{aligned}
\mu_{l}= & \left(\left(m_{N N}-\lambda\right)(\epsilon+\lambda)+d_{N N}\right)+\sum_{j=1}^{N-1}\left(m_{N j}(\epsilon+\lambda)+d_{N j}\right) \zeta_{l}^{j} \\
= & -\lambda^{2}+\frac{\rho \sigma}{2} \lambda\left[\frac{\mathrm{i}}{2}\left(e^{\mathrm{i}(\alpha+\beta)}\left(Z_{N}^{(l-2 k)}-Z_{N}^{(2 k)}\right)-e^{-\mathrm{i}(\alpha+\beta)}\left(Z_{N}^{(2 k+l)}-Z_{N}^{(2 k)}\right)\right)\right. \\
& \left.+\left(Z_{N}^{(l)}-1\right) \sin (\alpha-\beta)-\frac{2 \epsilon}{\rho \sigma}\right]+\epsilon \sigma \rho \frac{\mathrm{i}}{2}\left(e^{\mathrm{i}(\alpha+\beta)}\left(Z_{N}^{(l-2 k)}-Z_{N}^{(2 k)}\right)-e^{-\mathrm{i}(\alpha+\beta)}\left(Z_{N}^{(2 k+l)}-Z_{N}^{(2 k)}\right)\right) .
\end{aligned}
$$

Here, we use the shorthand form $Z_{N}^{(n)}=Z_{N}^{(n)}(1)$. If any of the $\mu_{l}$ vanishes, the determinant in (A.25) vanishes, as well. Thus, $\mu_{l}(\lambda)=0$ is giving the quadratic equation which determine the Lyapunov spectrum of the linearized system (6.15)-(6.16) around a phase-locked solution and with a base topology, given by the adjacency matrix $A$, having constant in-degree. Note that with the result in Sec. 6.2.1, the complex order parameter can be further simplified

$$
Z_{N}^{(n)}=R_{N}^{(n)}=\frac{1}{P}\left(\sum_{j=1}^{P} \cos \left(n j \frac{2 \pi}{N}\right)\right)
$$

Using the latter equation and well-known trigonometric equations, the following relations are derived:

$$
\begin{aligned}
Z_{N}^{(l-2 k)}-Z_{N}^{(2 k)} & =\frac{1}{P}\left(\sum_{j=1}^{P}\left(\cos \left((l-2 k) j \frac{2 \pi}{N}\right)-\cos \left(2 k j \frac{2 \pi}{N}\right)\right)\right), \\
& =-\frac{2}{P}\left(\sum_{j=1}^{P} \sin \left(l j \frac{\pi}{N}\right) \sin \left((l-4 k) j \frac{\pi}{N}\right)\right), \\
& =-\frac{2}{P}\left(\sum_{j=1}^{P} \sin \left(l j \frac{\pi}{N}\right)\left(\sin \left(l j \frac{\pi}{N}\right) \cos \left(4 k j \frac{\pi}{N}\right)-\cos \left(l j \frac{\pi}{N}\right) \sin \left(4 k j \frac{\pi}{N}\right)\right)\right),
\end{aligned}
$$

and analogously

$$
Z_{N}^{(l+2 k)}-Z_{N}^{(2 k)}=-\frac{2}{P}\left(\sum_{j=1}^{P} \sin \left(l j \frac{\pi}{N}\right)\left(\sin \left(l j \frac{\pi}{N}\right) \cos \left(4 k j \frac{\pi}{N}\right)+\cos \left(l j \frac{\pi}{N}\right) \sin \left(4 k j \frac{\pi}{N}\right)\right)\right) .
$$


Combining these relations, we find

$$
\begin{gathered}
L(\alpha, \beta, l, k)=\frac{\mathrm{i}}{2}\left(e^{\mathrm{i}(\alpha+\beta)}\left(Z_{N}^{(l-2 k)}-Z_{N}^{(2 k)}\right)-e^{-\mathrm{i}(\alpha+\beta)}\left(Z_{N}^{(2 k+l)}-Z_{N}^{(2 k)}\right)\right) \\
=\frac{2 \sin (\alpha+\beta)}{P} \sum_{j=1}^{P} \sin ^{2}\left(l j \frac{\pi}{N}\right) \cos \left(4 k j \frac{\pi}{N}\right) \\
+\mathrm{i} \frac{2 \cos (\alpha+\beta)}{P} \sum_{j=1}^{P} \sin \left(l j \frac{\pi}{N}\right) \cos \left(l j \frac{\pi}{N}\right) \sin \left(4 k j \frac{\pi}{N}\right) .
\end{gathered}
$$

\section{A.7 Stability of lifted one-cluster states}

For an arbitrary duplex equilibrium of the form $\phi_{i}^{\mu}=\Omega t+a_{i}^{\mu}$ with $a_{k}^{1}=\left(0, \frac{2 \pi}{N} k, \ldots,(N-1) \frac{2 \pi}{N} k\right)^{T}$ and $a_{k}^{2}=\mathbf{a}_{k}^{1}-\Delta a$ we start with the linearized system (4) of the main text. This can also be written in the block matrix form

$$
\left(\begin{array}{c}
\dot{\delta} \dot{\phi}^{1} \\
\dot{\delta \dot{\phi}^{2}} \\
\dot{\delta \dot{\kappa}^{1}} \\
\dot{\delta \dot{\kappa}^{2}}
\end{array}\right)=\left(\begin{array}{cccc}
A_{1} & m_{1} \mathbb{I}_{N} & B_{1} & 0 \\
m_{2} \mathbb{I}_{N} & A_{2} & 0 & B_{2} \\
C_{1} & 0 & -\epsilon \mathbb{I}_{N^{2}} & 0 \\
0 & C_{2} & 0 & -\epsilon \mathbb{I}_{N^{2}}
\end{array}\right)\left(\begin{array}{c}
\delta \phi^{1} \\
\delta \phi^{2} \\
\delta \kappa^{1} \\
\delta \kappa^{2}
\end{array}\right)
$$

with $\left(\delta \phi^{\mu}, \delta \kappa^{\mu}\right)^{T}=\left(\delta \phi_{1}^{\mu}, \ldots, \delta \phi_{N}^{\mu}, \delta \kappa_{11}^{\mu}, \ldots, \delta \kappa_{1 N}^{\mu}, \delta \kappa_{21}^{\mu}, \ldots, \delta \kappa_{N N}^{\mu}\right)^{T}$, the matrices $A^{\mu}, B^{\mu}$, and $C^{\mu}$ follow from system (4) of the main text, and $m_{1}, m_{2} \in \mathbb{R}$. With the help of Schur's decomposition the characteristic equation for the linearized system takes the form

$$
\operatorname{det}\left(\begin{array}{cc}
\left(\lambda \mathbb{I}_{N}-A_{1}\right)(\lambda+\epsilon)-B_{1} C_{1} & (\lambda+\epsilon)^{2\left(N^{2}-N\right)}=0 \\
-(\lambda+\epsilon) m_{2} \mathbb{I}_{N} & \left(\lambda \mathbb{I}_{N}-A_{2}\right)(\lambda+\epsilon)-m_{1} \mathbb{I}_{N} C_{2}
\end{array}\right)=0 .
$$

The second equation has the block matrix form which is required from Proposition 8.3.3. All blocks can be diagonalized and commute since they all possess a cyclic structure; compare Lemma 4.2.1. Thus, we are allowed to apply Proposition 8.3.3 which we use in order to diagonalize the matrix in Eq. (A.28). For the diagonalized matrix we find the following equations for the diagonal elements $\mu_{i}$

$$
(\lambda+\epsilon)^{2} m_{1} m_{2}-\left(p_{i}^{1}\left(\lambda ; \alpha^{11}, \beta^{1}, \alpha^{12}, \sigma^{12}\right)-\mu_{i}\right)\left(p_{i}^{2}\left(\lambda ; \alpha^{22}, \beta^{2}, \alpha^{21}, \sigma^{21}\right)-\mu_{i}\right)=0
$$

where $i=1, \ldots, N, p_{i}^{\mu}\left(\lambda ; \alpha^{\mu \mu}, \beta^{\mu}\right)$ is a second order polynomial in $\lambda$ which depends continuously on $\alpha$ and $\beta$ as well as functionally on the type of the one-cluster state. For every $i \in\{1, \ldots, N\}$, these equations will give us two eigenvalues $\mu_{i, 1}$ and $\mu_{i, 2}$ for the matrix in Eq. (A.28) depending on $\lambda$ and the system parameters. Thus, we can write Eq. (A.29) as

$$
\left(\mu_{i}-\mu_{i, 1}(\lambda ; \boldsymbol{\alpha}, \boldsymbol{\beta}, \boldsymbol{\sigma})\right)\left(\mu_{i}-\mu_{i, 2}(\lambda ; \boldsymbol{\alpha}, \boldsymbol{\beta}, \boldsymbol{\sigma})\right)=0
$$


where $\boldsymbol{\alpha}, \boldsymbol{\beta}, \boldsymbol{\sigma}$ represent all system parameter chosen for (2.25)-(2.26). In order to find the eigenvalue $\lambda$ of the linearized system (A.28) one of the eigenvalues $\mu$ has to vanish. This means that we have to find $\lambda$ such that Eq. (A.29) equals

$$
\mu_{i}\left(\mu_{i}-\mu_{i, 2}(\lambda ; \alpha, \beta, \sigma)\right)=0
$$

which is equivalent to finding $\lambda$ such that the following quartic equation is solved

$$
p_{i}^{1}\left(\lambda ; \alpha^{11}, \beta^{1}, \alpha^{12}, \sigma^{12}\right) p_{i}^{2}\left(\lambda ; \alpha^{22}, \beta^{2}, \alpha^{21}, \sigma^{21}\right)-(\lambda+\epsilon)^{2} m_{1} m_{2}=0 .
$$

Note that here the diagonal elements of $A_{1}$ are slightly different from those in Prop. 4.2.2 but they do not affect the result, i.e., the diagonal element equals $\rho_{i}\left(\alpha^{11}, \beta^{1}\right)-m_{1}\left(\alpha_{12}\right)$. The same holds true for $A_{2}$. Thus, with the two possible eigenvalues $\rho_{i, 1,2}\left(\alpha^{\mu \mu}, \beta^{\mu}\right)$ for the monoplex system from Corr. 4.2.3 one finds the following quartic equation which give the Lyapunov exponents for the lifted duplex one-cluster

$$
\begin{aligned}
& {\left[\left(\lambda-\rho_{i, 1}\left(\alpha^{11}, \beta^{1}\right)\right) \cdot\left(\lambda-\rho_{i, 2}\left(\alpha^{11}, \beta^{1}\right)\right)+m_{1}(\lambda+\epsilon)\right] \times} \\
& {\left[\left(\lambda-\rho_{i, 1}\left(\alpha^{22}, \beta^{2}\right)\right)\left(\lambda-\rho_{i, 2}\left(\alpha^{22}, \beta^{2}\right)\right)+m_{2}(\lambda+\epsilon)\right]-(\lambda+\epsilon)^{2} m_{1} m_{2}=0 .}
\end{aligned}
$$

In case of an antipodal monoplex one-cluster given by Eq. (2) of the main text with $a_{i} \in\{0, \pi\}$, then the set of Lyapunov exponents $\delta$ for Eq. (4)in the main text is given by

In the case of a duplex antipodal one-cluster state given by Eq. (2) of the main text with $a_{i}^{1} \in\{0, \pi\}$ and $a_{i}^{2}=a_{i}^{1}-\Delta a$, Eq. (3) possesses the following set of eigenvalues

$$
\oint_{\text {Duplex }}=\left\{-\epsilon,\left(\lambda_{i, 1}, \lambda_{i, 2}, \lambda_{i, 3}, \lambda_{i, 4}\right)_{i=1, \ldots, N}\right\}
$$

where $\lambda_{i, 1, \ldots, 4}$ solve the following $N$ quartic equations

$$
(\lambda+\epsilon)^{2} m_{1} m_{2}-\left[\left(\lambda-\rho_{i, 1}^{1}\right) \cdot\left(\lambda-\rho_{i, 2}^{1}\right)+m_{1}(\lambda+\epsilon)\right]\left[\left(\lambda-\rho_{i, 1}^{2}\right)\left(\lambda-\rho_{i, 2}^{2}\right)+m_{2}(\lambda+\epsilon)\right]=0,
$$

with $m_{1}=\sigma^{12} \cos \left(\Delta a+\alpha^{12}\right), m_{2}=\sigma^{21} \cos \left(\Delta a-\alpha^{21}\right)$ and the eigenvalues $\rho_{i, 1,2}^{\mu} \equiv \rho_{i, 1,2}\left(\alpha^{\mu \mu}, \beta^{\mu}\right)$ for the monoplex system, see Corollary 4.2.3. 


\section{A.8 Example for a complex adjacency matrix}

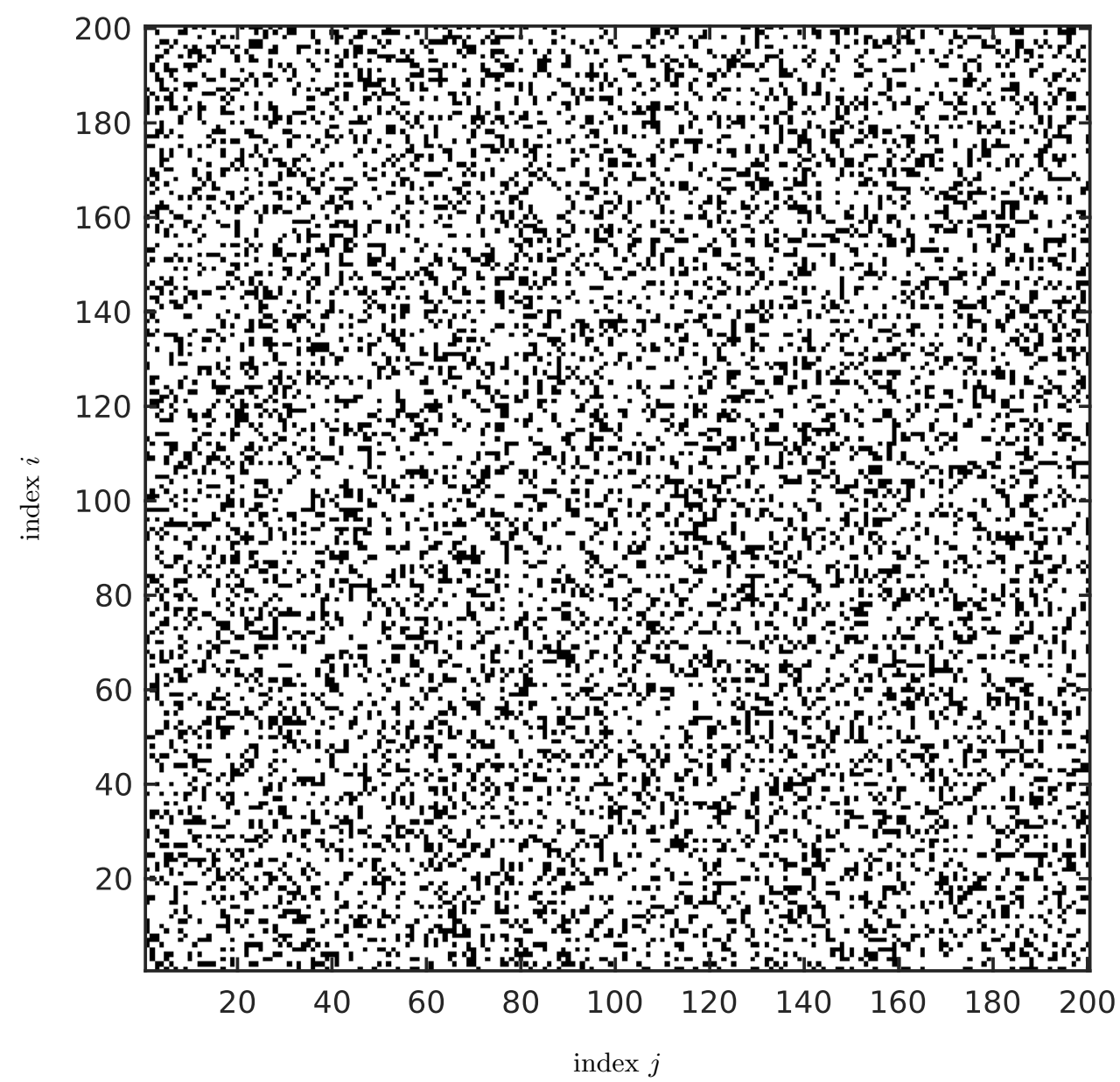

Figure A.1: Adjacency matrix of a connected, directed random network of $N=200$ nodes with constant row sum $r=50$. The illustration shows the adjacency matrix where black and white refer to whether a link between two nodes exist or not, respectively.

The adjacency matrix displayed in Fig. A.1 is obtained by the following procedure. For each node $i$ of the $N$ nodes, $r$ links are randomly (with uniform distribution) picked from the set consisting of all links from a node $j \neq i$ to node $i$. This procedure results in a random directed network with $N$ nodes and constant row sum (in-degree) $r$. After the procedure we test if the resulting network is connected [KOR18], see also 2.1. 


\section{List of Figures}

2.1 Illustration of different networks with a total number of 12 nodes: (a) globally coupled network, (b) nonlocally coupled network (coupling range $P=3$ ), (c) locally coupled network (coupling range $P=1$ ), and (d) multiplex network consisting of two layers for which one layer is globally coupled and the other layer has a nonlocally coupled network structure. . . . . . . . . . . . . . . . . .

2.2 The plasticity function $-\sin (\Delta \phi+\beta)$ and corresponding plasticity rules. (a) $\beta=-\frac{\pi}{2}$, (b) $\beta=0$, (c) $\beta=\frac{\pi}{2}$. Figure taken from [BER19, BER19a]. . . . . . . . . . . . . .

3.1 Plasticity function $W\left(\Delta t_{i j}\right)$ for $\tau_{p}=2, \tau_{d}=5, c_{p}=2, c_{d}=1$.6. Figure taken from [ROE19a]. 27

3.2 Evolution of the coupling matrix $\kappa_{i j}(t)$ starting from random initial conditions and converging to a completely synchronous state. Panel (a) shows initial coupling matrix, (b) the coupling matrix after the transient $t=2000 \mathrm{~ms}$. Raster plot of spiking times at the beginning of simulations (c) and after the transient (d). The asymptotic state $(\mathrm{b}, \mathrm{d})$ is a completely synchronized spiking with all coupling weights $\kappa_{i j}$ potentiated to $k_{\max }$. Other parameters $N=200, \tau_{p}=2, \tau_{d}=5, c_{p}=2, c_{d}=1.6$, and $\kappa_{\max }=1.5$. Figure modified from $[\mathrm{ROE} 19 \mathrm{a}] . \ldots \ldots \ldots \ldots \ldots$

3.3 Evolution of the coupling matrix $\kappa_{i j}(t)$ starting from random initial conditions and converging to frequency clusters hierarchical in size. Panel (a) shows initial coupling matrix, (b) the coupling matrix after the transient $t=5600 \mathrm{~ms}$, and (c) $t=20000 \mathrm{~ms}$. (d-f) Corresponding raster plots of spike times. The asymptotic state $(\mathrm{c}, \mathrm{f})$ is a hierarchical cluster state with the coupling weights $\kappa_{i j}$ potentiated to $k_{\max }$ within each cluster and small or zero otherwise. Other parameters as in Fig. 3.2. The oscillators are ordered accordingly to their mean frequency. Figure modified from [ROE19a]. . . . . . . . . .

3.4 Formation of individual clusters over time (corresponds to the dynamical scenario in Fig. 3.3). The dashed and solid curves depict the time course of the mean coupling within the small and big clusters, respectively. Figure taken from [ROE19a]. . . . . . .

3.5 Example of a three-cluster state for $N=500, \tau_{p}=2, \tau_{d}=5, c_{p}=2, c_{d}=1.6$, and $\kappa_{\max }=1.5$ with a random initial distribution of $\kappa_{i j}$ in $[0,0.75]$. Figure taken from $[$ ROE19a]. . . . . . . . . . . . . . . . . . . . . .

3.6 Coupling matrices for $t=10000 \mathrm{~ms}$ and different amplitudes of independent random input $I$ (see Eq. (3.4)). (a) $I=0.005$, (b) $I=0.01$, (c) $I=0.02$, (d) $I=0.05$ and (e) $\mathrm{I}=0.07$. All other parameters as in Fig. 3.5. Figure modified from [ROE19a]. . . . . . . . . . .

3.7 (a) Difference between synchronization frequencies of the two clusters for different size of the smaller cluster $N_{s}$. (b) Time until cluster fusion for different initial size of the smaller cluster $N_{s}$. Figure modified from [ROE19a]. . . . . . . . . . . . .

3.8 Evolution of the coupling matrix for $N=50$ and the number of neurons $N_{s}=8(\mathrm{a}-\mathrm{c})$ and $N_{s}=9(\mathrm{~d}-\mathrm{f})$ in the small cluster. In panels (a-c) the clusters are stable, while in (d-f) they are merging to one synchronous cluster. $(\mathrm{g}, \mathrm{h})$ Time courses of the spiking synchronization frequencies of small ( $N_{s}$ neurons) and large ( $N_{b}$ neurons) clusters depicted by dashed and solid curves, respectively, for (g) $N_{s}=8$ and $N_{b}=42$ and (h) $N_{s}=9$ and $N_{b}=41$. Parameter $\kappa_{\max }=1.0$. Figure modified from [ROE19a]. . . . . . 
3.9 Mean synaptic activity $S(t)$ of the neural population in the case of stable two cluster state. Panel (a) shows the dynamics of $S(t)$ on the time interval of $12 \mathrm{~s}$, where modulation of the amplitude (blue line) is visible, while the fast oscillations are not recognized on this timescale. The maximum amplitude corresponds to the two clusters being synchronised, while the low amplitude corresponds to the clusters being out of phase. Panel (b) shows the zoom of a small time interval. The modulation takes place on the timescale which is two orders of magnitude larger than the individual spikes of $S(t)$ as well as individual neural spikes in both clusters. Cluster frequencies $\omega_{1}=0.065012 \mathrm{kHz}$ and $\omega_{2}=0.065416 \mathrm{kHz}$. The corresponding period of modulation is $T \approx 2.5$ s. Figure modified from $[$ ROE19a]. . . . . . . . . . . . . . .

3.10 (a) Update function $G(\varphi)$ for $\tau_{p}=2, \tau_{d}=5, c_{p}=2$, and $c_{d}=1.6$. (b) Schematic spiking of two oscillators with spike time difference $\Delta T$ and periods close to $T$. Figure modified from $[\mathrm{ROE} 19 \mathrm{a}] . \ldots \ldots \ldots \ldots \ldots \ldots$

3.11 Phase portraits of model (3.10)-(3.11) for (a) monostable regime of complete synchronization; (b) co-existence of stable synchronized and clustered states; and (c) bifurcation moment of transition between the phase portraits illustrated in (a) and (b). The basins of attraction of the synchronized regime (point $S$ ), clustered state (limit cycle indicated by thick black curve) and the saddle fixed point $\left(\varphi^{*}, \sigma^{*}\right)$ are depicted by gray, blue, and white colors, respectively. The nullclines of the system and stable and unstable manifolds of the saddle point are indicted by the thin gray and black curves, respectively. Parameters (a) $\omega=0.037 \mathrm{kHz}$, (b) $\omega=0.06 \mathrm{kHz}$, (c) $\omega \approx 0.455 \mathrm{~Hz}$, and the other parameters $\tau_{p}=2, \tau_{d}=5, c_{p}=2, c_{d}=1.6$, and $\varepsilon=0.08$. Figure modified

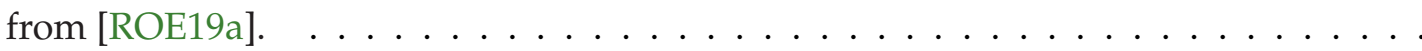

3.12 Dynamics of the phase difference between the clusters $\varphi_{H H}$ and mean inter-cluster coupling $\sigma_{H H}$ for the solutions of the Hodgkin-Huxley system (3.1)-(3.3) for different initial conditions. $N=50$ with $N_{s}=7$ neurons in the small cluster and $N_{b}=43$ in the big one. Red orbits converge to the regime of complete synchronization, and blue trajectories lead to a stable two-cluster solutions. The nullclines of the phenomenological model are shown in gray. Other parameters: $\tau_{p}=2, \tau_{d}=5, c_{p}=2, c_{d}=1.6$, and $\kappa_{\max }=1.5$. Figure taken from $[\mathrm{ROE} 19 \mathrm{a}] . \ldots \ldots \ldots \ldots$

3.13 Panel (a): system (3.10)-(3.11). White region: stable periodic solution coexisting with a stable fixed point, case II. Black region: globally stable fixed point, case I. Grey region: globally stable periodic solution with $\sigma=0$. Panel (b): original system (3.1)-(3.3). White: stable two-clusters (white); black: stable synchrony and no stable clusters; grey: decoupling of all neurons. Other parameters $\tau_{p}=2, \tau_{d}=5, N=50, N_{s}=7$, and $\kappa_{\max }=1$. Figure modified from $[$ ROE19a $] \ldots \ldots \ldots \ldots$

4.1 Illustration of the three types of one-cluster solutions given by (4.3)-(4.4) for an ensemble of 50 oscillators. One-cluster solutions (a) of splay type $\left(R_{2}(\mathbf{a})=0\right)$ for $\alpha=0.3 \pi, \beta=0.1 \pi$, (b) of antipodal type $\left(R_{2}(\mathbf{a})=1\right)$, for $\alpha=0.2 \pi, \beta=-0.95 \pi$ and (c) of double antipodal type satisfying condition (iii) of Proposition 4.1.1 with $m=30$ for $\alpha=0.3 \pi, \beta=-0.15 \pi$. Figure taken from [BER19]. . . . . . . . . . . .

4.2 Illustration of the family of solutions $S$ (a) $N=2$, (b) $N=3$, (c) $N=4$. Figure taken from $[$ BER19] . . . . . . . . . . . . . . . . . . . . . . . 
4.3 Stability diagrams for rotating-wave clusters depending on the parameters $\alpha$ and $\beta$ are shown. The regions are colored according to numerical simulation. Blue regions correspond to stable solutions while yellow regions correspond to unstable solutions. The black dashed lines show to the borders of stability determined by Corollary 4.2.3. Parameter $\epsilon=0.01$ is fixed fo all simulations. (a) $k=1$, (b) $k=N / 2$, (c) $k=N / 4$. Figure taken from $[$ BER19] . . . . . . . . . . . . . . . . . . . . . . .

4.4 Stability diagram for splay and antipodal one-cluster solutions depending on the parameters $\alpha$ and $\beta$ are shown. The regions are colored according numerical eigenvalues of the Jacobian 4.9-4.10. Blue areas correspond to stable while yellow areas correspond to unstable regions. Parameter $\epsilon=0.01$ is fixed in all simulations. (a) Splay solution as in Fig. 4.1(a), (b) Anti-phase solution as in Fig. 4.1(b). Figure modified from [BER19].

4.5 The regions of stability for antipodal and rotating-wave states are presented in $(\alpha, \beta)$ parameter space for different values of $\epsilon$. Coloured and hatched areas refer to stable regions for these states as indicated in the legend. White areas refer to region where these one-cluster states are unstable. (a) $\epsilon=0$; (b) $\epsilon=0.01$; (c) $\epsilon=0.1$; (d) $\epsilon=1$. Figure taken from $[$ BER19a]. . . . . . . . . . . . . . . . . . . . .

4.6 Heteroclinic orbits between several steady states in a system of 3 and 100 adaptively coupled phase oscillators. (a) The time series for the relative phases $\theta_{12}$ (solid lines) and $\theta_{13}$ (dashed lines) for $N=3$ are shown. Lines with the same colour correspond to the same trajectories. Panel $(b, c)$ show time series for the second moment order parameter $R_{2}(\phi(t))$ as well as a schematic illustration of the observed heteroclinic connections (right) for (b) $N=3$ and (c) $N=100$. Parameter values: $\epsilon=0.01, \alpha=0.4 \pi$, and $\beta=-0.15 \pi$. Figure taken from [BER19a]. . . . . . . . . . . . .

5.1 Three-frequencycluster of splay type at $t=10000$. (a) Coupling weights represented as a graph (left) and as a coupling matrix (right). In the graph representation, the dynamical nodes are represented by red nodes and the edges are coloured with respect to the coupling weight. Red and blue refer to positive and negative coupling weights, respectively. Light and dark colors refer to weak and strong coupling weights, respectively. (b) Distribution of the phases $\phi_{i}$ for each of the three clusters. Each node represents one oscillator and is coloured with respect to the cluster to which it belongs. Parameter values: $\epsilon=0.01, \alpha=0.3 \pi, \beta=0.23 \pi, \omega=0$, and $N=100$. Figure modified from $[$ BER19a] . . . . . . . . . . . . . . . . . . . . . . .

5.2 Three different types of multicluster states at $t=10000$ with $N=100$ and $\epsilon=0.01$. For all types, the coupling matrix (left), distribution of the phases (middle), and time series of representative phase oscillators from each cluster (right) are presented. In the plot of the phase distribution, each node represents one oscillator and is colored with respect to the cluster to which it belongs. The time series are shown after subtracting the average linear growth $\phi_{i, \mu}(t)-\left\langle\Omega_{\mu}\right\rangle t$. The colouring of the time series (shaded for visibility) of a representative phase oscillator from one cluster is in accordance with the pictures in the middle panel. (a) Splay type 3-cluster for $\alpha=0.3 \pi, \beta=0.23 \pi$; (b) Antipodal type 3-cluster for $\alpha=0.3 \pi, \beta=-0.53 \pi$; (c) Mixed type 2-cluster for $\alpha=0.3 \pi$, $\beta=-0.4 \pi$. Figure taken from $[$ BER19a] . . . . . . . . . . . . . . 
5.3 Three-cluster of splay type. (a) Coupling weights at $t=10000$ showing three clusters; (b) Distribution of the phases within each cluster; space-time raster plot; (c) Average frequency of oscillators; each plateau corresponds to one cluster; (d) Oscillator phases $\phi_{i}(t)$ at fixed time $t=10000$. Parameter values: $\epsilon=0.01, \alpha=0.3 \pi, \beta=0.23 \pi$, and

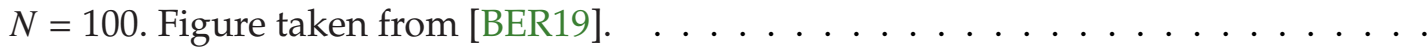

5.4 The figures show all one- and two-cluster solutions of splay type for the system (5.1)-(5.2). For this, the frequency differences $\Delta \Omega_{12}$ are displayed corresponding to the equations (4.6) and (5.7). The dotted lines (black) indicate unstable solutions while the solid lines (blue) indicate stable solutions. Here, every second solution is plotted for the sake of visibility. Parameter values: (a) $N=20, \epsilon=0.01$; (b) $N=50, \epsilon=0.01$; (c) $N=50, \epsilon=0.001$; (d) $N=50, \epsilon=0.1 ; \alpha=0.3 \pi$ is fixed for all panels. Figure taken from [BER19] . . . . . . . . . . . . . . . . . . . . . . . .

5.5 For the case of two-cluster states of splay type, the critical value $\epsilon_{c}$ of time-separation parameter $\epsilon$ is plotted as a function of $\gamma=\alpha-\beta$ for different cluster sizes $n_{1}=N_{1} / N$. The function is given explicitly by Eq. (5.10). Figure taken from [BER19a]. . . . . . . .

5.6 Three-cluster of antipodal type. (a) Coupling weights at $t=10000$ showing three clusters; (b) Distribution of the phases within each cluster; space-time raster plot; (c) Average frequency of oscillators; each plateau corresponds to one cluster; (d) Oscillator phases $\phi_{i}(t)$ at fixed time $t=10000$. Parameter values: $\epsilon=0.01, \alpha=0.3 \pi$, $\beta=-0.53 \pi$, and $N=100$. Figure taken from [BER19] . . . . . . . . . . .

5.7 For 3-cluster solution from Fig. 5.6, panel (a) shows time series of an oscillator from one of the clusters after subtracting the average linear growth $\phi_{\mu, i}(t)-\left\langle\Omega_{\mu}\right\rangle t$. The black dashed lines show the corresponding analytic results from the asymptotic expansion in Proposition 5.4.1. (b) Power spectrum of the time series given in (a). Figure modified from [BER19] . . . . . . . . . . . . . . . . . . . . . . . .

5.8 Two-cluster solutions (upper panels) and one-cluster solutions (lower panels) of antipodal type given by the asymptotic expansion in Corollary 5.4.2 and Proposition 4.1.1, respectively. For this, the difference of the frequencies $\Delta \Omega_{12}^{(1)}$ is displayed corresponding to (5.15) and (4.6). The dotted lines (black) indicate unstable solutions while the solid lines (blue) indicate stable solutions. Here, every second solution is plotted for the sake of visibility. The insets show a blow-up of the interval $[-\epsilon, \epsilon]$. Parameter values: (a) $N=20, \epsilon=0.01$; (b) $N=50, \epsilon=0.01$; $\alpha=0.3 \pi$ is fixed for all panels. Figure taken from $[$ BER19]. . . . . . . . . . . . . . . . .

5.9 2-Cluster solution of mixed type. (a) Coupling weights at $t=10000$ showing two clusters, (b) Distribution of the phases within each cluster, space-time representation. (c) Average frequency of each oscillator, (d) Oscillator phases $\phi_{i}$ for fixed time $t=$ 10000. Parameter values: $\epsilon=0.01, \alpha=0.3 \pi, \beta=-0.4 \pi, N=100$. Figure taken from $[$ BER19]. . . . . . . . . . . . . . . . . . . . . .

5.10 For mixed type 2-cluster solution from Fig. 5.9, panel (a) shows time series of an oscillator from one of the clusters after subtracting the average linear growth $\phi_{\mu, i}(t)-\left\langle\Omega_{\mu}\right\rangle t$. The black dashed lines show the corresponding analytic results from the asymptotic expansion in Proposition 5.5.1. (b) Power spectrum of the time series given in (a). Figure moified from [BER19]. 
5.11 Two-cluster solutions of mixed type (upper panels) and one-cluster solutions (lower panels) of either splay or antipodal type given by the asymptotic expansion in Corollary 5.5.2 and Proposition 4.1.1, respectively. For this, the difference of the frequencies $\Delta \Omega_{12}^{(1)}$ is displayed corresponding to (5.17) and (4.6). The dotted lines (black) indicate unstable solutions while the solid lines (blue) indicate stable solutions. Here, every second solution is plotted for the sake of visibility. The insets show a blow-up of the interval $[-\epsilon, \epsilon]$. Parameter values: (a) $N=20, \epsilon=0.01$; (b) $N=50, \epsilon=0.01 ; \alpha=0.3 \pi$ is fixed for all panels. Figure taken from [BER19]. . . . . . . . . . . . .

5.12 Stability diagram for the one-cluster and two-cluster solution of the splay type depending on the parameters $\alpha$ and $\beta$. Yellow region corresponds to the instability of both solutions, dark blue to the stability of both solutions, and light-blue to the stability of only the one-cluster solution. Parameter $\epsilon=0.01$ is fixed in all simulations. Figure taken from $[$ BER19]. . . . . . . . . . . . . . . . . . .

6.1 Illustration for two types of one-cluster states. The panels $(a, c)$ show the asymptotic coupling matrices and $(b, d)$ snapshots of the phases at a fixed time. Results for the one-cluster states of antipodal type are presented in $(\mathrm{a}, \mathrm{b})$ where $\alpha=0.19 \pi, \beta=-0.66 \pi$ and of splay type in (c,d) where $\alpha=0.35 \pi, \beta=0.01 \pi$. Parameters: $N=100, P=20$, $\epsilon=0.01$. Figure modified from [?] . . . . . . . . . . . . . . .

6.2 Schematic figure illustrating the definition of multicluster and subnetworks induced by groups of nodes with the same average frequency. The full network (left) consists of $N=20$ nodes and has a nonlocal ring structure with $P=4$. The colors of the nodes indicate their average frequencies. Clusters are shown by the equally colored nodes that form connected sub-networks. Even though the two blue groups I and II possess the same averaged frequencies, they form two different clusters, since they are not connected. Figure taken from $[$ BER20c] $\ldots \ldots \ldots \ldots \ldots$

6.3 Illustration of the different types of multicluster states. The panels $(\mathrm{a}, \mathrm{d}, \mathrm{g})$ show the coupling matrix, $(\mathrm{b}, \mathrm{e}, \mathrm{h})$ phase snapshots and $(\mathrm{c}, \mathrm{f}, \mathrm{i})$ average frequencies. $(\mathrm{a}-\mathrm{c})$ : antipodal two-cluster for $\alpha=0.23 \pi, \beta=-0.56 \pi$; (d-f): splay two-cluster for $\alpha=0.19 \pi$, $\beta=-0.45 \pi$; (g-i): antipodal five-cluster (I, II denote the two connected components of the red and the blue clusters) for $\alpha=0.3 \pi, \beta=-0.53 \pi$. Parameters: $N=100, P=20$, $\epsilon=0.01$. Figure taken from $[$ BER20c] $\ldots \ldots \ldots \ldots \ldots$

6.4 Map of regimes for one- and multicluster states of antipodal and splay type in $(\alpha, \beta)$ parameter space. Parameters: $N=100, P=20, \epsilon=0.01$. The horizontal black line at $\alpha=0.1$ shows the location for the parameter $\beta$ where the emergence of solitary states is analyzed, see Fig. 6.7 in Sec. 6.3. Figure taken from [BER20c] . . . . . . . . . . . .

6.5 Illustration of solitary states. The panels $(\mathrm{a}, \mathrm{d})$ show coupling matrix, $(\mathrm{b}, \mathrm{e})$ phase snapshots, and (c,f) average frequencies. (a-c): single solitary state for $\alpha=0.1 \pi$, $\beta=-0.3 \pi$; (d-f): three uncoupled solitary states for $\alpha=0.15 \pi, \beta=-0.41 \pi$. Parameters: $N=100, P=20, \epsilon=0.01$. Figure modified from [BER20c]. . . . . . . . . . .

6.6 Stability of one-cluster states for different wavenumbers $k$ and coupling ranges $P$. Regions of stability for the one-cluster states are colored in blue, while instability in yellow. The borders of stability (black dashed lines) are obtained from the eigenvalues (6.17). Parameters are as follows: (a) $P=10, k=1$; (b) $P=10, k=4$; (c) $P=10, k=25$; (d) $P=5, k=1$; (e) $P=20, k=1$; and (f) $P=25, k=1$. The other parameters are $N=50$ and $\epsilon=0.01$. Figure modified from [BER20c] . . . . . . . . . . . 
6.7 Phase portraits for two-dimensional system (6.20)-(6.21). The graphics show the two classes of asymptotic states that are equilibria (colored nodes) and periodic solutions (colored lines). The stability properties of the individual asymptotic states are indicated by the coloring where the blue refers to stable and the red (dashed) to unstable states. In addition, several trajectories are plotted in black including those close to the stable and unstable manifold of the equilibria. The nullclines are displayed as gray lines. For the different panels parameter $\beta$ is varied as shown in Fig. 6.4: (a) $\beta=-0.601 \pi$; (b) $\beta=-0.599 \pi$; (c) $\beta=-0.58 \pi$; (d) $\beta=-0.5515 \pi$; (e) $\beta=-0.5 \pi$; (f) $\beta=-0.08 \pi$; (g) $\beta=-0.0563 \pi$; and (h) $\beta=0.05 \pi$. The other parameters are $\alpha=0.1 \pi$ and $\epsilon=0.01$. Figure modified from $[$ BER20c] . . . . . . . . . . . . .

7.1 The figure shows the master stability function for the equations (7.11)-(7.12). Regions belonging to negative Lyapunov exponents are colored blue. The one dimensional curve where at least one eigenvalue of (7.15) has zero real part is given as a black dotted line. Parameter: (a) $\beta=-0.8 \pi$, (b) $\beta=-0.2 \pi$, (c) $\beta=-0.02 \pi$, (d) $\beta=0.05 \pi$, (e) $\beta=0.1 \pi$, and (f) $\beta=0.98 \pi$. The other parameters are $\alpha=0.3 \pi$ and $\epsilon=0.01$. . . . . 106

7.2 Results for an adiabatic continuation in the coupling constant $\sigma$ of the full synchronous solution $\phi_{i}=0$ and $\kappa_{i j}=-\sin (\beta)$ for a globally coupled network of $N=200$ oscillators (7.11)-(7.12). The cluster parameter $R_{C}$ for different values of $\sigma$ is presented. As an inset, for the three values (a) $\sigma=0.002$, (b) $\sigma=0.006$, and (c) $\sigma=0.025$, the master stability function, the phases $\phi_{i}$ of the final state, and the frequencies of the oscillators $\Omega_{i}$ are plotted. The oscillators are sorted as in Fig 5.2. If more than or equal to ten (for numerical convenience) oscillators have the same frequency (coherent groups) all nodes of this group are plotted as circles and with a respective color. All other oscillators are plotted as an asterisk. The master function parameter $\tilde{\mu}=\sigma \mu$ for the subnetworks induced by the coherent groups are plotted together with the master stability function. The colors of each coherent group agree in all three plots. Parameters: $\alpha=0.49 \pi, \beta=0.88 \pi, \epsilon=0.01$.

7.3 Results for an adiabatic continuation in the coupling constant $\sigma$ of the full synchronous solution $\phi_{i}=0$ and $\kappa_{i j}=-\sin (\beta)$ for a complex network (see Appendix A.8) of $N=200$ oscillators (7.11)-(7.12). The cluster parameter $R_{C}$ for different values of $\sigma$ is presented. As an inset, for the three values (a) $\sigma=0.003$, (b) $\sigma=0.007$, and (c) $\sigma=0.019$, the master stability function, the phases $\phi_{i}$ of the final state, and the frequencies of the oscillators $\Omega_{i}$ are plotted. If more than or equal to ten ()for numerical convenience) oscillators have the same frequency (coherent groups) all nodes of this group are plotted as circles and with a respective color. All other oscillators are plotted as an asterisk. The master function parameter $\tilde{\mu}=\sigma \mu$ for the subnetworks induced by the coherent groups are plotted together with the master stability function. The colors of each coherent group agree in all three plots. Parameters: $\alpha=0.49 \pi, \beta=0.88 \pi$,

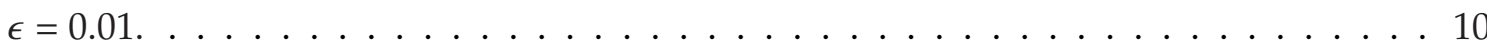


8.1 Different duplex states of Eq. (4.3) $(L=2)$ for an ensemble of 50 oscillators in each layer with color-coded coupling weights $\kappa_{i j}^{\mu}$ (upper panels, color code as in Fig.1), phases $\phi_{j}^{\mu}$ (lower panels): Duplex one-cluster states (a) of lifted splay type $\left(R_{2}\left(\mathbf{a}^{\mu}\right)=0\right)$ for $\alpha^{12 / 21}=0.3 \pi, \sigma^{12 / 21}=0.07$; (b) of lifted antipodal type $\left(R_{2}\left(\mathbf{a}^{\mu}\right)=1\right)$ for $\alpha^{12}=0.3 \pi$, $\alpha^{21}=0.75 \pi, \sigma^{12 / 21}=0.62$; (c) of double antidodal type (not a lifted state) for $\alpha^{12 / 21}=$ $0.05 \pi, \sigma^{12 / 21}=0.28$; (d) of lifted splay type for $\alpha^{12}=0.3 \pi, \alpha^{21}=0.4 \pi, \sigma^{12 / 21}=0.8$, and $\epsilon=0.01$. In the lower panels phase differences between the two layers are indicated by $\Delta a \equiv a_{i}^{1}-a_{i}^{2}$, and between the two new antipodal states (c) by $\psi^{1}, \psi^{2}$. Figure taken from [BER19b] . . . . . . . . . . . . . . . . . . . . . .

8.2 Birth of double antipodal state in a duplex network $(N=12)$ for a wide range of inter-layer coupling strength $\sigma=\sigma^{12}=\sigma^{21}$. The solid lines are the temporal averages for the second moment order parameter $R_{2}$ of the individual layers (layer 1: black, layer 2: red). The error bars for $\sigma<\sigma_{c}$ denote the standard deviation of the temporal evolution of $R_{2}$. The dashed horizontal lines represent the unique values of $R_{2}$ for the double antipodal state in a monoplex network. The plot was obtained by adiabatic continuation of a duplex double antipodal state (see inset) in both directions starting from $\sigma=0.5$. Parameters: $\alpha^{11 / 22}=0.3 \pi, \alpha^{12 / 21}=0.05, \beta^{1}=0.1 \pi, \beta^{2}=-0.95 \pi$, and $\epsilon=0.01$. Figure taken from $[$ BER19b] . . . . . . . . . . . . . . . . .

8.3 The figure shows the range $\Delta \omega$ vs where duplex one-cluster states in general (gray) and of the form presented in Fig. 3 of the main text (red) can be found. For this the system (8.7), (8.8) is integrated numerically for 10 different random uniform distributions of the natural frequencies in the interval $[-\Delta \omega, \Delta \omega]$. The results are obtained by adiabatic continuation starting with the phase clusters found for $\Delta \omega=0$ (see Fig. 3 of the main text). Duplex one-cluster states of double antipodal type with (a) $\sigma=0.5, \Delta=0.02$ and (b) $\sigma=0.5, \Delta=0.07$ are shown as insets. Parameters: $\alpha^{11 / 22}=0.3 \pi, \alpha^{12 / 21}=0.05, \beta^{1}=0.1 \pi, \beta^{2}=-0.95 \pi, \epsilon=0.01$, and $N=12$. Figure taken from [BER19b] . . . . . . . . . . . . . . . . . . . . .

8.4 The figure shows the standard deviation $\rho$ where duplex one-cluster states in general (gray) and of the form presented in Fig. 3 of the main text (red) can be found. For this the system (8.7), (8.8) is integrated numerically for 10 different random normal distributions of the natural frequencies with standard deviation $\rho$ and zero mean. The results are obtained by adiabatic continuation starting with the phase clusters found for $\rho=0$ (see Fig. 3 of the main text). A duplex one-cluster state of double antipodal type with $\sigma=0.5, \Delta=0.02$ is shown as an inset. Parameters: $\alpha^{11 / 22}=0.3 \pi$, $\alpha^{12 / 21}=0.05, \beta^{1}=0.1 \pi, \beta^{2}=-0.95 \pi, \epsilon=0.01$, and $N=12$. Figure taken from [BER19b]. 117

8.5 Regions of stability (blue) and instability (white) of the lifted antipodal state in the $\left(\alpha^{22}, \beta^{2}\right)$ parameter plane for different values of interlayer coupling (indicated by different blue shading) $\sigma^{21}$, where regions of stronger coupling $\sigma^{21}$ (lighter blue) include such of weaker $\sigma^{21}$ (darker blue). Stability regions for single-layer antipodal clusters are indicated by red hatched areas. The inter-layer coupling is considered as (a) unidirectional $\left(\sigma^{12}=0\right)$ and $(\mathrm{b})$ bidirectional $\left(\sigma^{12}=\sigma^{21}\right)$. Parameters: $\alpha^{11}=0.2 \pi$, $\beta^{1}=-0.8 \pi, \alpha^{12}=0, \alpha^{21}=0.3 \pi$, and $\epsilon=0.01$. Figure taken from [BER19b]. . . . . .

A.1 Adjacency matrix of a connected, directed random network of $N=200$ nodes with constant row sum $r=50$. The illustration shows the adjacency matrix where black and white refer to whether a link between two nodes exist or not, respectively. . . . . 



\section{List of Tables}

4.1 The table summarizes the values for $n$th order parameter for the phase-locked solutions introduced in Definition 4.1.1 . . . . . . . . . . . . . . . . . 42 



\section{Acknowledgement}

First of all, I would like to thank Prof. Dr. Dr. h.c. Eckehard Schöll, PhD and PD Dr. Serhiy Yanchuk for introducing me to the exciting field of adaptive networks and the theory of nonlinear dynamics. I also thank for their supervision, valuable help, and support by suggestions and questions regarding my research. I appreciate their encouragement to frequent visits of international conferences which enabled me to profit from the exchange of knowledge within the research community from a very early stage on.

I am very much in debt to Dr. Jakub Sawicki who provided guidance during the research and writing of this thesis. Furthermore, I would like to thank Prof. Dr. Vladimir Nekorkin and Dr. Dmitry Kasatkin for the fruitful collaboration which resulted in some of the results presented in Chapter 4 and 5. Likewise, I am grateful for the collaboration with PD Dr. Oleksandr Popovych. I owe thanks to all the current, former, and visiting members of the Schöll group and the Yanchuk group for the wonderful working atmosphere. In particular, I thank Dr. Iryna Omelchenko, Prof. Dr. Anna Zakharova, Phd, Prof. Dr. Yuri Maistrenko, Dr. Stefan Ruschel, Dr. Simona Olmi, Dr. Dmitry Puzyrev, Dr. Ewandson Lameu, Dr. Vander Freitas, Jan Fialkowski, Giulia Ruzzene, Florian Stelzer, Nour Eldine Hanbali, Narges Chinichian, Michael Lindner, Deniz Nikitin, Maria Masoliver, Franziska Beckschulte, Danila Semenov, my students Jacopo Zurbuch, Sören Nagel, Philippe Lehmann, Simon Vock, Max Thiele, Amy Searle, Fenja Drauschke, Johanna Czech, Lucas Kluge, Moritz Gerster, Vera Röhr, and my RISE students Bricker Ostler and Alicja Polanska for exciting discussions and fruitful collaborations. Additionally, I owe thanks to Prof. Dr. Sarika Jalan and Dr. Saptarshi Gosh for insightful discussions. Further, I would like to express my thanks to Andrea Schulze who helped more than once with her tremendous knowledge on the universities' bureaucracy to pass administrative challenges and to Peter Orlowski who could always help me when things went wrong with the IT.

I would like to thank Prof. Dr. Alessandro Torcini for preparing the third assessment of this thesis and Prof. Dr. Dieter Breitschwerdt for chairing the defense of this thesis.

This work was supported in the framework of the DFG-RSF (Deutsche ForschungsgemeinschaftRussian Science Foundation) project: "Complex dynamical networks: effects of heterogeneous, adaptive and time delayed couplings" under SCHO 307/15-1 and YA 225/3-1. Further, I would like to thank the DAAD (Deutscher Akademischer Austauschdienst) which provided funding within the RISE program to host two students for a summer internship at the AG Schöll. I also thank all members of the IRTG (International Research Training Group) 1740 and the SFB (Collorative Research Center) 910 for supporting me to take part in their exciting events and conferences which were a fruitful ground to develop new ideas and get into contact with a lot of inspiring people.

Last but certainly not least I thank my family for their constant support during the whole time of my studies and in particular I thank my dear girlfriend Veronika for encouraging me to finalize this thesis, for supporting me when things went slowly or got stuck and for taking me out of my scientific bubble sometimes. 



\section{Bibliography}

[ABB00] L. F. Abbott and S. Nelson: Synaptic plasticity: taming the beast, Nat. Neurosci. 3, 1178-1183 (2000).

[ABR88] R. Abraham, J. E. Marsden, and T. Ratiu: Manifolds, Tensor Analysis, and Applications (Springer, New York, 1988).

[ABR04] D. M. Abrams and S. H. Strogatz: Chimera states for coupled oscillators, Phys. Rev. Lett. 93, 174102 (2004).

[ABR08] D. M. Abrams, R. E. Mirollo, S. H. Strogatz, and D. A. Wiley: Solvable model for chimera states of coupled oscillators, Phys. Rev. Lett. 101, 084103 (2008).

[ACE05] J. A. Acebrón, L. L. Bonilla, C. J. Pérez Vicente, F. Ritort, and R. Spigler: The Kuramoto model: A simple paradigm for synchronization phenomena, Rev. Mod. Phys. 77, 137-185 (2005).

[AVA18] V. Avalos-Gaytán, J. A. Almendral, I. Leyva, F. Battiston, V. Nicosia, V. Latora, and S. Boccaletti: Emergent explosive synchronization in adaptive complex networks, Phys. Rev. E 97, 042301 (2018).

[ALB02a] R. Albert and A. L. Barabási: Statistical mechanics of complex networks, Rev. Mod. Phys. 74, 47-97 (2002).

[ALC19] P. Alcami and A. E. Pereda: Beyond plasticity: the dynamic impact of electrical synapses on neural circuits, Nat. Rev. Neurosci. 20, 263-271 (2019).

[AMA17] R. Amato, A. Díaz-Guilera, and K. K. Kleineberg: Interplay between social influence and competitive strategical games in multiplex networks, Sci. Rep. 7, 7087 (2017).

[AMA17a] R. Amato, N. E. Kouvaris, M. San Miguel, and A. Díaz-Guilera: Opinion competition dynamics on multiplex networks, New J. Phys. 19, 123019 (2017).

[AMU19] K. Amunts, A. C. Knoll, T. Lippert, C. M. A. Pennartz, P. Ryvlin, A. Destexhe, V. K. Jirsa, E. D'Angelo, and J. G. Bjaalie: The human brain projec-synergy between neuroscience, computing, informatics, and brain-inspired technologies, PLoS Biol. 17, e3000344 (2019).

[AND16] R. G. Andrzejak, C. Rummel, F. Mormann, and K. Schindler: All together now: Analogies between chimera state collapses and epileptic seizures, Sci. Rep. 6, 23000 (2016).

[AND17] R. G. Andrzejak, G. Ruzzene, and I. Malvestio: Generalized synchronization between chimera states, Chaos 27, 053114 (2017).

[AOK09] T. Aoki and T. Aoyagi: Co-evolution of phases and connection strengths in a network of phase oscillators, Phys. Rev. Lett. 102, 034101 (2009).

[AOK11] T. Aoki and T. Aoyagi: Self-organized network of phase oscillators coupled by activitydependent interactions, Phys. Rev. E 84, 066109 (2011). 
[AOK12] T. Aoki and T. Aoyagi: Scale-free structures emerging from co-evolution of a network and the distribution of a diffusive resource on it, Phys. Rev. Lett. 109, 208702 (2012).

[AOK15] T. Aoki: Self-organization of a recurrent network under ongoing synaptic plasticity, Neural Networks 62, 11-19 (2015).

[AOK15a] T. Aoki, K. Yawata, and T. Aoyagi: Self-organization of complex networks as a dynamical system, Phys. Rev. E 91, 012908 (2015).

[AOK16] T. Aoki, L. E. C. Rocha, and T. Gross: Temporal and structural heterogeneities emerging in adaptive temporal networks, Phys. Rev. E 93, 040301 (2016).

[ALV14] C. Alvarado-Rojas, M. Valderrama, A. Fouad-Ahmed, H. Feldwisch-Drentrup, M. Ihle, C. A. Teixeira, F. Sales, A. Schulze-Bonhage, C. Adam, A. Dourado, S. Charpier, V. Navarro, and M. Le Van Quyen: Slow modulations of high-frequency activity (40-140 hz) discriminate preictal changes in human focal epilepsy, Sci. Rep. 4, 4545 (2014).

[ARE08] A. Arenas, A. Díaz-Guilera, J. Kurths, Y. Moreno, and C. Zhou: Synchronization in complex networks, Phys. Rep. 469, 93-153 (2008).

[ASH92b] P. Ashwin and J. W. Swift: The dynamics of $n$ weakly coupled identical oscillators, J. Nonlinear Sci. 2, 69-108 (1992).

[ASH08] P. Ashwin, O. Burylko, and Y. Maistrenko: Bifurcation to heteroclinic cycles and sensitivity in three and four coupled phase oscillators, Physica D 237, 454-466 (2008).

[ASH15] P. Ashwin and O. Burylko: Weak chimeras in minimal networks of coupled phase oscillators, Chaos 25, 013106 (2015).

[ASH16a] P. Ashwin, C. Bick, and O. Burylko: Identical phase oscillator networks: Bifurcations, symmetry and reversibility for generalized coupling, Front. Appl. Math. Stat. 2 (2016).

[ASH16] P. Ashwin, S. Coombes, and R. Nicks: Mathematical frameworks for oscillatory network dynamics in neuroscience, J. Math. Neurosci. 6:2, 2 (2016).

[ASH16b] P. Ashwin and A. Rodrigues: Hopf normal form with $S_{N}$ symmetry and reduction to systems of nonlinearly coupled phase oscillators, Physica D 325, 14-24 (2016).

[ASH19] A. Ashourvan, Q. K. Telesford, T. Verstynen, J. M. Vettel, and D. S. Bassett: Multi-scale detection of hierarchical community architecture in structural and functional brain networks, PLoS ONE 14, e0215520 (2019).

[ASL17] M. M. Asl, A. Valizadeh, and P. A. Tass: Dendritic and axonal propagation delays determine emergent structures of neuronal networks with plastic synapses, Sci. Rep. 7, 39682 (2017).

[ASL18] M. M. Asl, A. Valizadeh, and P. A. Tass: Delay-induced multistability and loop formation in neuronal networks with spike-timing-dependent plasticity, Sci. Rep. 8, 12068 (2018).

[ASL18a] M. M. Asl, A. Valizadeh, and P. A. Tass: Dendritic and axonal propagation delays may shape neuronal networks with plastic synapses, Front. Physiol. 9, 1849 (2018).

[ASS11] S. Assenza, R. Gutiérrez, J. Gómez-Gardeñes, V. Latora, and S. Boccaletti: Emergence of structural patterns out of synchronization in networks with competitive interactions, Sci. Rep. 1, 99 (2011). 
[BAL09] A. G. Balanov, N. B. Janson, D. E. Postnov, and O. V. Sosnovtseva: Synchronization: From Simple to Complex (Springer, Berlin, 2009).

[BAR99a] A. L. Barabási and R. Albert: Emergence of scaling in random networks, Science 286, 509 (1999).

[BAR11d] M. Barthélemy: Spatial Networks, Phys. Rep. 499, 1-101 (2011).

[BAS06a] D. S. Bassett and E. T. Bullmore: Small-world brain networks, Neuroscientist 12, 512-523 (2006).

[BAS17] D. S. Bassett and O. Sporns: Network neuroscience, Nat. Neurosci. 20, 353 EP (2017), Review Article.

[BAS18] D. S. Bassett, P. Zurn, and J. I. Gold: On the nature and use of models in network neuroscience, Nat. Rev. Neurosci. 19, 566-578 (2018).

[BAT17] F. Battiston, V. Nicosia, M. Chavez, and V. Latora: Multilayer motif analysis of brain networks, Chaos 27, 047404 (2017).

[BAU20] F. Baumann, P. Lorenz-Spreen, I. M. Sokolov, and M. Starnini: Modeling echo chambers and polarization dynamics in social networks, Phys. Rev. Lett. 124, 048301 (2020).

[BAZ02] M. Bazhenov, I. Timofeev, M. Steriade, and T. J. Sejnowski: Model of thalamocortical slow-wave sleep oscillations and transitions to activated states, J. Neurosci. 22, 8691-8704 (2002).

[BEL97a] C. C. Bell, V. Z. Han, Y. Sugawara, and K. Grant: Synaptic plasticity in a cerebellum-like structure depends on temporal order., Nature 387, 278-281 (1997).

[BEL04a] I. Belykh, V. N. Belykh, and M. Hasler: Blinking model and synchronization in small-world networks with a time-varying coupling, Physica D 195, 188-206 (2004).

[BEL11a] I. Belykh and M. Hasler: Mesoscale and clusters of synchrony in networks of bursting neurons, Chaos 21, 016106 (2011).

[BEN04a] M. V. Bennett and R. S. Zukin: Electrical coupling and neuronal synchronization in the mammalian brain, Neuron 41, 495-511 (2004).

[BEN16] B. Bentley, R. Branicky, C. L. Barnes, Y. L. Chew, E. Yemini, E. T. Bullmore, P. E. Vétes, and W. R. Schafer: The multilayer connectome of caenorhabditis elegans, PLOS Comput. Biol. 12, e1005283 (2016).

[BER19d] B. K. Bera, S. Rakshit, and D. Ghosh: Intralayer synchronization in neuronal multiplex network, Eur. Phys. J. Spec. Top. 228, 2441-2454 (2019).

[BER19a] R. Berner, J. Fialkowski, D. V. Kasatkin, V. I. Nekorkin, S. Yanchuk, and E. Schöll: Hierarchical frequency clusters in adaptive networks of phase oscillators, Chaos 29, 103134 (2019).

[BER19] R. Berner, E. Schöll, and S. Yanchuk: Multiclusters in networks of adaptively coupled phase oscillators, SIAM J. Appl. Dyn. Syst. 18, 2227-2266 (2019).

[BER19e] M. Bertolero and D. S. Bassett: How matter becomes mind, Sci. Am., 18-25 (2019). 
[BER20c] R. Berner, A. Polanska, E. Schöll, and S. Yanchuk: Solitary states in adaptive nonlocal oscillator networks, Eur. Phys. J. Spec. Top. (2020), arXiv: 1911.00320.

[BER19b] R. Berner, J. Sawicki, and E. Schöll: Birth and stabilization of phase clusters by multiplexing of adaptive networks, Phys. Rev. Lett. 124, 088301 (2020).

[BI98] G. q. Bi and M. m. Poo: Synaptic modifications in cultured hippocampal neurons: Dependence on spike timing, synaptic strength, and postsynaptic cell type, J. Neurosci. 18, 10464-10472 (1998).

[BI01] G. q. Bi and M. m. Poo: Synaptic modification by correlated activity: Hebb's postulate revisited, Annu. Rev. Neurosci. 24, 139-166 (2001).

[BI16] H. Bi, X. Hu, S. Boccaletti, X. Wang, Y. Zou, Z. Liu, and S. Guan: Coexistence of quantized, time dependent, clusters in globally coupled oscillators, Phys. Rev. Lett. 117, 204101 (2016).

[BIC15] C. Bick and E. A. Martens: Controlling chimeras, New J. Phys. 17, 033030 (2015).

[BIC16b] C. Bick and P. Ashwin: Chaotic weak chimeras and their persistence in coupled populations of phase oscillators, Nonlinearity 29, 1468-1486 (2016).

[BIC16] C. Bick, P. Ashwin, and A. Rodrigues: Chaos in generically coupled phase oscillator networks with nonpairwise interactions, Chaos 26, 094814 (2016).

[BIR19] T. Birkoben, M. Drangmeister, F. Zahari, S. Yanchuk, P. Hövel, and H. Kohlstedt: Bifurcation without parameters in a chaotic system with a memristive element, arXiv:1906.02445 (2019).

[BLA13] K. Blaha, J. Lehnert, A. Keane, T. Dahms, P. Hövel, E. Schöll, and J. L. Hudson: Clustering in delay-coupled smooth and relaxational chemical oscillators, Phys. Rev. E 88, 062915 (2013).

[BLA19] K. A. Blaha, K. Huang, F. Della Rossa, L. M. Pecora, M. Hossein-Zadeh, and F. Sorrentino: Cluster synchronization in multilayer networks: A fully analog experiment with $l c$ oscillators with physically dissimilar coupling, Phys. Rev. Lett. 122, 014101 (2019).

[BLI93] T. V. P. Bliss and G. L. Collingridge: A synaptic model of memory: long-term potentiation in the hippocampus, Nature 361, 31-39 (1993).

[BOC02] S. Boccaletti, J. Kurths, G. Osipov, D. L. Valladares, and C. S. Zhou: The synchronization of chaotic systems, Phys. Rep. 366, 1-101 (2002).

[BOC14] S. Boccaletti, G. Bianconi, R. Criado, C. I. del Genio, J. Gómez-Gardeñes, M. Romance, I. Sendiña Nadal, Z. Wang, and M. Zanin: The structure and dynamics of multilayer networks, Phys. Rep. 544, 1-122 (2014).

[BOC16] S. Boccaletti, J. A. Almendral, S. Guan, I. Leyva, Z. Liu, I. Sendiña-Nadal, Z. Wang, and Y. Zou: Explosive transitions in complex networks' structure and dynamics: Percolation and synchronization, Phys. Rep. 660 (2016).

[BOC18] S. Boccaletti, A. N. Pisarchik, C. I. del Genio, and A. Amann: Synchronization: From Coupled Systems to Complex Networks (Cambridge University Press, Cambridge, 2018).

[BOL98] B. Bollobás: Modern Graph Theory (Springer, New York, NY, 1998). 
[BON09] M. Bonnin: Waves and patterns in ring lattices with delays, Physica D 238, 77-87 (2009).

[BOE17a] C. Börgers: An Introduction to Modeling Neuronal Dynamics (Springer, Cham, 2017).

[BOY04] S. Boyd and L. Vandenberghe: Convex Optimization (Cambridge University Press, March (2004)).

[BRE97] P. C. Bressloff, S. Coombes, and B. de Souza: Dynamics of a ring of pulse-coupled oscillators: Group-theoretic approach, Phys. Rev. Lett. 79, 2791 (1997).

[BRE17a] M. Breakspear: Dynamic models of large-scale brain activity, Nat. Neurosci. 20, 340-352 (2017).

[BRO88a] T. H. Brown, P. F. Chapman, E. W. Kairiss, and C. L. Keenan: Long-term synaptic

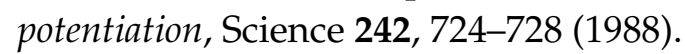

[BOT12] V. Botella-Soler and P. Glendinning: Emergence of hierarchical networks and polysynchronous behaviour in simple adaptive systems, Europhys. Lett. 97, 50004 (2012).

[BOT14] V. Botella-Soler and P. Glendinning: Hierarchy and polysynchrony in an adaptive network, Phys. Rev. E 89, 062809 (2014).

[BUC68] J. Buck and E. Buck: Mechanism of rhythmic synchronous flashing of fireflies: Fireflies of southeast asia may use anticipatory time-measuring in synchronizing their flashing, Science 159, 1319-1327 (1968).

[BUL09] E. T. Bullmore and O. Sporns: Complex brain networks: graph theoretical analysis of structural and functional systems, Nat. Rev. Neurosci. 10, 186-198 (2009).

[BUR11] O. Burylko and A. Pikovsky: Desynchronization transitions in nonlinearly coupled phase oscillators, Physica D 240, 1352-1361 (2011).

[BUR18] O. Burylko, A. Mielke, M. Wolfrum, and S. Yanchuk: Coexistence of hamiltonian-like and dissipative dynamics in rings of coupled phase oscillators with skew-symmetric coupling, SIAM J. Appl. Dyn. Syst. 17, 2076-2105 (2018).

[CAL09a] M. Calamai, A. Politi, and A. Torcini: Stability of splay states in globally coupled rotators, Phys. Rev. E 80, 036209 (2009).

[CAP08a] N. Caporale and Y. Dan: Spike timing-dependent plasticity: A Hebbian learning rule, Annu. Rev. Neurosci. 31, 25-46 (2008).

[CAR13d] A. Cardillo, M. Zanin, J. Gòmez Gardeñes, M. Romance, A. Garcia del Amo, and S. Boccaletti: Modeling the multi-layer nature of the european air transport network: Resilience and passengers re-scheduling under random failures, Eur. Phys. J. ST 215, 23-33 (2013).

[CAT08] H. Câteau, K. Kitano, and T. Fukai: Interplay between a phase response curve and spiketiming-dependent plasticity leading to wireless clustering, Phys. Rev. E 77, 051909 (2008).

[CHA14a] V. K. Chandrasekar, J. H. Sheeba, B. Subash, M. Lakshmanan, and J. Kurths: Adaptive coupling induced multi-stable states in complex networks, Physica D 267, 36-48 (2014). 
[CHA17a] S. Chakravartula, P. Indic, B. Sundaram, and T. Killingback: Emergence of local synchronization in neuronal networks with adaptive couplings, PLoS ONE 12, e0178975 (2017).

[CHE19b] B. Chen, J. R. Engelbrecht, and R. E. Mirollo: Dynamics of the Kuramoto-Sakaguchi oscillator network with asymmetric order parameter, Chaos 29, 013126 (2019).

[CHO09] C. U. Choe, T. Dahms, P. Hövel, and E. Schöll: Controlling synchrony by delay coupling in networks: from in-phase to splay and cluster states, Phys. Rev. E 81, 025205(R) (2010).

[CHO18] T. Chouzouris, I. Omelchenko, A. Zakharova, J. Hlinka, P. Jiruska, and E. Schöll: Chimera states in brain networks: empirical neural vs. modular fractal connectivity, Chaos 28, 045112 (2018).

[CLO10] C. Clopath, L. Büsing, E. Vasilaki, and W. Gerstner: Connectivity reflects coding: a model of voltage-based STDP with homeostasis, Nat. Neurosci. 13, 344-352 (2010).

[COM03] A. Compte, M. V. Sanchez-Vives, D. A. McCormick, and X. J. Wang: Cellular and network mechanisms of slow oscillatory activity $(<1 \mathrm{~Hz})$ and wave propagations in a cortical network model, J. Neurophysiol. 89, 2707 (2003).

[CON04] B. W. Connors and M. A. Long: Electrical synapses in the mammalian brain, Annu. Rev. Neurosci. 27, 393-418 (2004).

[COO16] S. Coombes and R. Thul: Synchrony in networks of coupled non-smooth dynamical systems: Extending the master stability function, Eur. J. Appl. Math. 27, 904-922 (2016).

[COS07] L. d. F. Costa, F. A. Rodrigues, G. Travieso, and P. R. Villas Boas: Characterization of complex networks: A survey of measurements, Adv. Phys. 56, 167-242 (2007).

[CUR16] S. Curti and J. O'Brien: Characteristics and plasticity of electrical synaptic transmission, BMC Cell Biol. 17 (2016).

[CVE97] D. Cvetkovic, P. Rowlinson, and S. Simic: Eigenspaces of Graphs (Cambridge University Press, 1997).

[DOE14] F. Dörfler and F. Bullo: Synchronization in complex networks of phase oscillators: A survey, Automatica 50, 1539-1564 (2014).

[DAH11b] T. Dahms: Synchronization in delay-coupled laser networks, Ph.D. thesis, Technische Universität Berlin (2011).

[DAH12] T. Dahms, J. Lehnert, and E. Schöll: Cluster and group synchronization in delay-coupled networks, Phys. Rev. E 86, 016202 (2012).

[DAI92a] H. Daido: Order function and macroscopic mutual entrainment in uniformly coupled limitcycle oscillators, Prog. Theor. Phys. 88, 1213-1218 (1992).

[DAI94] H. Daido: Generic scaling at the onset of macroscopic mutual entrainment in limit-cycle oscillators with uniform all-to-all coupling, Phys. Rev. Lett. 73, 760-763 (1994).

[DAM19] F. Damicelli, C. C. Hilgetag, M. T. Hütt, and A. Messé: Topological reinforcement as a principle of modularity emergence in brain networks, Netw. Neurosci. 3, 589-605 (2019). 
[DAN06] Y. Dan and M. m. Poo: Spike timing-dependent plasticity: from synapse to perception, Physiol. Rev. 86, 1033-1048 (2006).

[DAV79] P. J. Davis: Circulant matrices (Wiley, 1979).

[DE13] M. De Domenico, A. Solé-Ribalta, E. Cozzo, M. Kivelä, Y. Moreno, M. A. Porter, S. Gómez, and A. Arenas: Mathematical formulation of multilayer networks, Phys. Rev. X 3, 041022 (2013).

[DOM13] M. De Domenico, A. Solé-Ribalta, E. Cozzo, M. Kivelä, Y. Moreno, M. A. Porter, S. Gómez, and A. Arenas: Mathematical formulation of multilayer networks, Phys. Rev. X 3, 041022 (2013).

[DE15] M. De Domenico, V. Nicosia, A. Arenas, and V. Latora: Structural reducibility of multilayer networks, Nat. Commun. 6, 6864 (2015).

[DEC09] G. Deco, V. K. Jirsa, A. R. McIntosh, O. Sporns, and R. Kötter: Key role of coupling, delay, and noise in resting brain fluctuations, Proc. Natl. Acad. Sci. U.S.A. 106, 10302-10307 (2009).

[DIP12] M. Dipoppa, M. Krupa, A. Torcini, and B. S. Gutkin: Splay states in finite pulse-coupled networks of excitable neurons, SIAM J. Appl. Dyn. Syst. 11, 864-894 (2012).

[LEL08] P. De Lellis, M. Bernardo, and F. Garofalo: Synchronization of complex networks through local adaptive coupling, Chaos 18, 037110 (2008).

[LEL09] P. De Lellis, M. di Bernardo, and F. Garofalo: Decentralized Adaptive Control for Synchronization and Consensus of Complex Networks, in Modelling, Estimation and Control of Networked Complex Systems, edited by A. Chiuso, L. Fortuna, M. Frasca, A. Rizzo, L. Schenato, and S. Zampieri (Springer Berlin Heidelberg, 2009), pp. 27-42.

[LEL10a] P. De Lellis, M. Bernardo, and G. Russo: On QUAD, Lipschitz, and Contracting Vector Fields for Consensus and Synchronization of Networks, IEEE Trans. Circuits Syst. I 58, 576-583 (2010).

[LEL10] P. De Lellis, M. di Bernardo, F. Garofalo, and M. Porfiri: Evolution of complex networks via edge snapping, IEEE Trans. Circuits Syst. I 57, 2132-2143 (2010).

[DU15] C. Du, W. Ma, T. Chang, P. Sheridan, and W. D. Lu: Biorealistic implementation of synaptic functions with oxide memristors through internal ionic dynamics, Adv. Funct. Mater. 25, 4290-4299 (2015).

[EGU05] V. M. Eguiluz, D. R. Chialvo, G. A. Cecchi, M. Baliki, and A. V. Apkarian: Scale-free brain functional networks., Phys. Rev. Lett. 94, 4 (2005).

[ERD59] P. Erdös and A. Rényi: On random graphs, Publ. Math. Debrecen 6, 290-297 (1959).

[ERD60] P. Erdös and A. Rényi: On the evolution of random graphs, Publ. Math. Inst. Hung. Acad. Sci 5, 17-61 (1960).

[ERM91] G. B. Ermentrout: An adaptive model for synchrony in the firefly pteroptyx malaccae, J. Math. Biol. 29, 571-585 (1991). 
[ERM19] G. B. Ermentrout, Y. Park, and D. Wilson: Recent advances in coupled oscillator theory, Philos. Trans. Royal Soc. A 377, 20190092 (2019).

[FEL11] J. Fell and N. Axmacher: The role of phase synchronization in memory processes, Nat. Rev. Neurosci. 12, 105-118 (2011).

[FEL12] D. E. Feldman: The spike-timing dependence of plasticity, Neuron 75, 556-571 (2012).

[FIE73] M. Fiedler: Algebraic connectivity of graphs, Czech. Math. J. 23, 298-305 (1973).

[FIE88] B. Fiedler: Global Bifurcation of Periodic Solutions with Symmetry (Springer-Verlag, Heidelberg, 1988).

[FIE89] M. Fiedler: Laplacian of graphs and algebraic connectivity, Banach Center Publications 25, 57-70 (1989).

[FIL08a] G. Filatrella, A. H. Nielsen, and N. F. Pedersen: Analysis of a power grid using a Kuramoto-like model, Eur. Phys. J. B 61, 485-491 (2008).

[FLU10b] V. Flunkert, S. Yanchuk, T. Dahms, and E. Schöll: Synchronizing distant nodes: a universal classification of networks, Phys. Rev. Lett. 105, 254101 (2010).

[FRO10] R. C. Froemke, J. J. Letzkus, B. M. Kampa, G. B. Hang, and G. J. Stuart: Dendritic synapse location and neocortical spike-timing-dependent plasticity, Front. Synaptic Neurosci. 2 (2010).

[FRO16] F. Fröhlich: Network Neuroscience (Academic Press, 2016).

[FRO18] N. S. Frolov, V. A. Maksimenko, V. V. Makarov, D. Kirsanov, A. E. Hramov, and J. Kurths: Macroscopic chimeralike behavior in a multiplex network, Phys. Rev. E 98, 022320 (2018).

[GOM13] S. Gómez, A. Díaz-Guilera, J. Gómez-Gardeñes, C. J. Pérez Vicente, Y. Moreno, and A. Arenas: Diffusion dynamics on multiplex networks, Phys. Rev. Lett. 110, 028701 (2013).

[GAM14] L. V. Gambuzza, A. Buscarino, S. Chessari, L. Fortuna, R. Meucci, and M. Frasca: Experimental investigation of chimera states with quiescent and synchronous domains in coupled electronic oscillators, Phys. Rev. E 90, 032905 (2014).

[GAV12] A. Gavalda, J. Duch, and J. Gómez-Gardeñes: Reciprocal interactions out of congestionfree adaptive networks, Phys. Rev. E 85, 026112 (2012).

[GER96] W. Gerstner, R. Kempter, J. L. von Hemmen, and H. Wagner: A neuronal learning rule for sub-millisecond temporal coding, Nature 383, 76-78 (1996).

[GER14a] W. Gerstner, W. M. Kistler, R. Naud, and L. Paninski: Neuronal Dynamics: From single neurons to networks and models of cognition (Cambridge University Press, July (2014)).

[GOM07] J. Gómez-Gardeñes, Y. Moreno, and A. Arenas: Paths to synchronization on complex networks, Phys. Rev. Lett. 98, 034101 (2007).

[GOM11a] J. Gómez-Gardeñes, S. Gómez, A. Arenas, and Y. Moreno: Explosive synchronization transitions in scale-free networks, Phys. Rev. Lett. 106, 128701 (2011). 
[GHO16] S. Ghosh, A. Kumar, A. Zakharova, and S. Jalan: Birth and death of chimera: Interplay of delay and multiplexing, Europhys. Lett. 115, 60005 (2016).

[GHO18] S. Ghosh, A. Zakharova, and S. Jalan: Non-identical multiplexing promotes chimera states, Chaos Solitons Fractals 106, 56-60 (2018).

[GIL04] A. Gillies and D. Willshaw: Models of the subthalamic nucleus: The importance of intranuclear connectivity, Med. Eng. and Phys. 26, 723-732 (2004).

[GIR02] M. Girvan and M. E. J. Newman: Community structure in social and biological networks, Proc. Natl. Acad. Sci. USA 99, 7821 (2002).

[GIR12] T. Girnyk, M. Hasler, and Y. Maistrenko: Multistability of twisted states in non-locally coupled kuramoto-type models, Chaos 22, 013114 (2012).

[GIU16] C. Giusti, R. Ghrist, and D. S. Bassett: Two's company, three (or more) is a simplex, J. Comp. Neurosci. 41, 1-14 (2016).

[GLE06] P. M. Gleiser and D. H. Zanette: Synchronization and structure in an adaptive oscillator network, Eur. Phys. J. B 53, 233-238 (2006).

[GOD01] C. Godsil and G. Royle: Algebraic Graph Theory (Springer-Verlag New York, 2001).

[GOL88a] M. Golubitsky and I. Stewart: Singularities and Groups in Bifurcation Theory. Volume 2, vol. 69 of Applied Mathematical Sciences (Springer-Verlag, New York, 1988).

[GOL16] M. Golubitsky and I. Stewart: Rigid patterns of synchrony for equilibria and periodic cycles in network dynamics, Chaos 26, 094803 (2016).

[GRA05] R. M. Gray: Toeplitz and circulant matrices: A review., Found. Trends Commun. Inf. Theory 2 (2005).

[GRA06] R. M. Gray: Toeplitz And Circulant Matrices: A Review, in Found. Trends Commun. Inf. Theory, edited by (Now Publishers Inc., Hanover, MA, USA, 2006), vol. 2, pp. 155-239.

[GRO06b] T. Gross, C. J. D. D'Lima, and B. Blasius: Epidemic dynamics on an adaptive network, Phys. Rev. Lett. 96, 208701 (2006).

[GRO08a] T. Gross and B. Blasius: Adaptive coevolutionary networks: a review, J. R. Soc. Interface 5, 259-271 (2008).

[GRO09] T. Gross and H. Sayama: Adaptive Networks (Springer-Verlag Berlin Heidelberg, 2009).

[GUC75] J. Guckenheimer: Isochrons and phaseless sets, J. Math. Biol. 1, 259-273 (1975).

[GUS15a] A. Gushchin, E. Mallada, and A. Tang: Synchronization of phase-coupled oscillators with plastic coupling strength, in Information Theory and Applications Workshop ITA 2015, San Diego, CA, USA, edited by (IEEE, February (2015)), pp. 291-300.

[GUT11] R. Gutiérrez, A. Amann, S. Assenza, J. Gómez-Gardeñes, V. Latora, and S. Boccaletti: Emerging meso- and macroscales from synchronization of adaptive networks, Phys. Rev. Lett. 107, 234103 (2011).

[GUZ13] P. Y. Guzenko, J. Lehnert, and E. Schöll: Application of adaptive methods to chaos control of networks of Rössler systems, Cybern. Phys. 2, 15-24 (2013). 
[HA16a] S. Y. Ha, S. E. Noh, and J. Park: Synchronization of kuramoto oscillators with adaptive couplings, SIAM J. Appl. Dyn. Syst. 15, 162-194 (2016).

[HAG12] A. M. Hagerstrom, T. E. Murphy, R. Roy, P. Hövel, I. Omelchenko, and E. Schöll: Experimental observation of chimeras in coupled-map lattices, Nat. Phys. 8, 658-661 (2012).

[HAL80] J. K. Hale: Ordinary differential equations (Krieger Publishing Company, Malabar, Florida, 1980).

[HAM07] C. Hammond, H. Bergman, and P. Brown: Pathological synchronization in Parkinson's disease: networks, models and treatments, Trends Neurosci. 30, 357-364 (2007).

[HAN93b] D. Hansel, G. Mato, and C. Meunier: Phase dynamics for weakly coupled hodgkin- huxley neurons, Europhys. Lett. 23, 367-372 (1993).

[HAN17] M. Hansen, F. Zahari, M. Ziegler, and H. Kohlstedt: Double-Barrier Memristive Devices for Unsupervised Learning and Pattern Recognition, Front. Neurol. Front. Neurosci. 11, 91 (2017).

[HAN18b] E. J. Hancock and G. A. Gottwald: Model reduction for kuramoto models with complex topologies, Phys. Rev. E 98, 012307 (2018).

[HAU09] C. Hauptmann and P. A. Tass: Cumulative and after-effects of short and weak coordinated reset stimulation: a modeling study, J. Neural Eng. 6, 016004 (2009).

[HEB49] D. Hebb: The Organization of Behavior: A Neuropsychological Theory (Wiley, New York, new edition edition, June (1949)).

[HEI11] S. Heiligenthal, T. Dahms, S. Yanchuk, T. Jüngling, V. Flunkert, I. Kanter, E. Schöll, and W. Kinzel: Strong and weak chaos in nonlinear networks with time-delayed couplings, Phys. Rev. Lett. 107, 234102 (2011).

[HEL20] F. Hellmann, P. Schultz, P. Jaros, R. Levchenko, T. Kapitaniak, J. Kurths, and Y. Maistrenko: Network-induced multistability through lossy coupling and exotic solitary states, Nat. Commun. 11, 592 (2020).

[HIZ15] J. Hizanidis, E. Panagakou, I. Omelchenko, E. Schöll, P. Hövel, and A. Provata: Chimera states in population dynamics: networks with fragmented and hierarchical connectivities, Phys. Rev. E 92, 012915 (2015).

[HOD52] A. L. Hodgkin and A. F. Huxley: A quantitative description of membrane current and its application to conduction and excitation in nerve, J. Physiol. 117, 500-544 (1952).

[HOL12] P. Holme and J. Saramäki: Temporal networks, Phys. Rep. 519, 97-125 (2012).

[HOL15a] P. Holme: Modern temporal network theory: a colloquium, Eur. Phys. J. B 88, 1-30 (2015).

[HOP96a] F. C. Hoppensteadt and E. M. Izhikevich: Synaptic organizations and dynamical properties of weakly connected neural oscillators, Biol. Cybern. 75, 117-127 (1996).

[HOP96] F. C. Hoppensteadt and E. M. Izhikevich: Synaptic organizations and dynamical properties of weakly connected neural oscillators ii. learning phase information, Biol. Cybern. 75, 129 -135 (1996). 
[HOP97] F. C. Hoppensteadt and E. M. Izhikevich: Weakly connected neural networks (Springer, New York, 1997).

[HOP99] F. C. Hoppensteadt and E. M. Izhikevich: Oscillatory neurocomputers with dynamic connectivity, Phys. Rev. Lett. 82, 2983-2986 (1999).

[HOR09b] Y. Horikawa and H. Kitajima: Duration of transient oscillations in ring networks of unidirectionally coupled neurons, Physica D 238, 216-225 (2009).

[HOE16] P. Hövel, J. Lehnert, A. Selivanov, A. L. Fradkov, and E. Schöll: Adaptively controlled synchronization of delay-coupled networks, in Control of Self-Organizing Nonlinear Systems, edited by E. Schöll, S. H. L. Klapp, and P. Hövel (Springer, Berlin, Heidelberg, 2016), chapter 3, pp. 47-63.

[HOE18] P. Hövel, A. Viol, P. Loske, L. Merfort, and V. Vuksanović: Synchronization in functional networks of the human brain, J. Nonlinear Sci. (2018).

[HUM06] M. D. Humphries, K. Gurney, and T. J. Prescott: The brainstem reticular formation is a small-world, not scale-free, network, Proceedings of the Royal Society B: Biological Sciences 273, 503-511 (2006).

[IGN15] M. Ignatov, M. Ziegler, M. Hansen, A. Petraru, and H. Kohlstedt: A memristive spiking neuron with firing rate coding, Front. Neurosci. 9, 376 (2015).

[ISE15a] T. M. Isele, B. Hartung, P. Hövel, and E. Schöll: Excitation waves on a minimal smallworld model, Eur. Phys. J. B 88, 104 (2015).

[ISE15] T. M. Isele and E. Schöll: Effect of small-world topology on wave propagation on networks of excitable elements, New J. Phys. 17, 023058 (2015).

[ITO01a] J. Ito and K. Kaneko: Spontaneous structure formation in a network of chaotic units with variable connection strengths, Phys. Rev. Lett. 88, 028701 (2001).

[ITO03] J. Ito and K. Kaneko: Spontaneous structure formation in a network of dynamic elements, Phys. Rev. E 67, 046226 (2003).

[IWA10a] M. Iwasa, K. Iida, and D. Tanaka: Hierarchical cluster structures in a one-dimensional swarm oscillator model, Phys. Rev. E 81, 046220 (2010).

[IWA10] M. Iwasa and D. Tanaka: Dimensionality of clusters in a swarm oscillator model, Phys. Rev. E 81, 066214 (2010).

[IZH03] E. M. Izhikevich: Simple model of spiking neurons, IEEE Trans. Neural Netw. 14, 1569$1572(2003)$.

[IZH04] E. M. Izhikevich: Which model to use for cortical spiking neurons?, IEEE Transactions on Neural Networks 15, 1063-1070 (2004).

[JAI01] S. Jain and S. Krishna: A model for the emergence of cooperation, interdependence, and structure in evolving networks, Proc. Natl. Acad. Sci. 98, 543-547 (2001).

[JAL16] S. Jalan and A. Singh: Cluster synchronization in multiplex networks, Europhys. Lett. 113, 30002 (2016). 
[JAL19] S. Jalan, A. Kumar, and I. Leyva: Explosive synchronization in frequency displaced multiplex networks, Chaos 29, 041102 (2019).

[JAR15] P. Jaros, Y. Maistrenko, and T. Kapitaniak: Chimera states on the route from coherence to rotating waves, Phys. Rev. E 91, 022907 (2015).

[JAR18] P. Jaros, S. Brezetsky, R. Levchenko, D. Dudkowski, T. Kapitaniak, and Y. Maistrenko: Solitary states for coupled oscillators with inertia, Chaos 28, 011103 (2018).

[JIR13] P. Jiruska, M. de Curtis, J. G. R. Jefferys, C. A. Schevon, S. J. Schiff, and K. Schindler: Synchronization and desynchronization in epilepsy: controversies and hypotheses, J. Physiol. 591.4, 787-797 (2013).

[JIR14] V. K. Jirsa, W. C. Stacey, P. P. Quilichini, A. I. Ivanov, and C. Bernard: On the nature of seizure dynamics, Brain 137, 2210 (2014).

[JOH18] R. A. John, F. Liu, N. A. Chien, M. R. Kulkarni, C. Zhu, Q. D. Fu, A. Basu, Z. Liu, and N. Mathews: Synergistic gating of electro-iono-photoactive $2 d$ chalcogenide neuristors: Coexistence of hebbian and homeostatic synaptic metaplasticity, Adv. Mater. 30, 1800220 (2018).

[KAN13] M. Kantner and S. Yanchuk: Bifurcation analysis of delay-induced patterns in a ring of Hodgkin-Huxley neurons, Phil. Trans. R. Soc. A 371, 20120470 (2013).

[KAP14] T. Kapitaniak, P. Kuzma, J. Wojewoda, K. Czolczynski, and Y. Maistrenko: Imperfect chimera states for coupled pendula, Sci. Rep. 4, 6379 (2014).

[KAR19] M. Karimian, D. Dibenedetto, M. Moerel, T. Burwick, R. L. Westra, P. De Weerd, and M. Senden: Effects of synaptic and myelin plasticity on learning in a network of kuramoto phase oscillators, Chaos 29, 083122 (2019).

[KAS16a] D. V. Kasatkin and V. I. Nekorkin: Dynamics of the phase oscillators with plastic couplings, Radiophysics and Quantum Electronics 58, 877-891 (2016).

[KAS17] D. V. Kasatkin, S. Yanchuk, E. Schöll, and V. I. Nekorkin: Self-organized emergence of multi-layer structure and chimera states in dynamical networks with adaptive couplings, Phys. Rev. E 96, 062211 (2017).

[KAS18a] D. V. Kasatkin and V. I. Nekorkin: The effect of topology on organization of synchronous behavior in dynamical networks with adaptive couplings, Eur. Phys. J. Spec. Top. 227, 1051 (2018).

[KAS18] D. V. Kasatkin and V. I. Nekorkin: Synchronization of chimera states in a multiplex system of phase oscillators with adaptive couplings, Chaos 28, 093115 (2018).

[KAS19] D. V. Kasatkin, V. Klinshov, and V. I. Nekorkin: Itinerant chimeras in an adaptive network of pulse-coupled oscillators, Phys. Rev. E 99, 022203 (2019).

[KEA12] A. Keane, T. Dahms, J. Lehnert, S. A. Suryanarayana, P. Hövel, and E. Schöll: Synchronisation in networks of delay-coupled type-I excitable systems, Eur. Phys. J. B 85, 407 (2012). 
[KEP02] A. Kepecs, M. C. W. van Rossum, S. Song, and J. Tegner: Spike-timing-dependent plasticity: common themes and divergent vistas, Biol. Cybern. 87, 446-458 (2002).

[KIM17a] S. S. Kim, H. Rouault, S. Druckmann, and V. Jayaraman: Ring attractor dynamics in the drosophila central brain., Science 356, 849 (2017).

[KIV14] M. Kivelä, A. Arenas, M. Barthélemy, J. P. Gleeson, Y. Moreno, and M. A. Porter: Multilayer networks, J. Complex Networks 2, 203-271 (2014).

[KLI15] V. Klinshov, L. Lücken, D. Shchapin, V. I. Nekorkin, and S. Yanchuk: Multistable jittering in oscillators with pulsatile delayed feedback, Phys. Rev. Lett. 114, 178103 (2015).

[KLI16a] P. Klimek, M. Diakonova, V. M. Eguiluz, M. San Miguel, and S. Thurner: Dynamical origins of the community structure of an online multi-layer society, New J. Phys. 18 (2016).

[KLI17] V. Klinshov, D. Shchapin, S. Yanchuk, M. Wolfrum, O. D’Huys, and V. I. Nekorkin: Embedding the dynamics of a single delay system into a feed-forward ring, Phys. Rev. E 96, 042217 (2017).

[KLI17a] V. Klinshov, S. Yanchuk, A. Stephan, and V. I. Nekorkin: Phase response function for oscillators with strong forcing or coupling, Europhys. Lett. 118, 50006 (2017).

[KLO11] P. E. Kloeden and M. Rasmussen: Nonautonomous dynamical systems (American Mathematical Society, 2011).

[KOH14] V. Kohar, P. Ji, A. Choudhary, S. Sinha, and J. Kurths: Synchronization in time-varying networks, Phys. Rev. E 90, 022812 (2014).

[KOP04] N. Kopell and G. B. Ermentrout: Chemical and electrical synapses perform complementary roles in the synchronization of interneuronal networks, Proc. Natl. Acad. Sci. U.S.A. 101, 15482-15487 (2004).

[KOR18a] W. Koroshetz, J. Gordon, A. Adams, A. Beckel-Mitchener, J. Churchill, G. Farber, M. Freund, J. Gnadt, N. S. Hsu, N. Langhals, S. Lisanby, G. Liu, G. C. Y. Peng, M. Steinmetz, E. Talley, and S. White: The State of the NIH BRAIN Initiative, J. Neurosci. 38, 6427-6438 (2018).

[KOR18] B. Korte and J. Vygen: Combinatorial Optimization (Springer, Berlin, Heidelberg, 2018).

[KOU14] N. E. Kouvaris, T. M. Isele, A. S. Mikhailov, and E. Schöll: Propagation failure of excitation waves on trees and random networks, Europhys. Lett. 106, 68001 (2014).

[KOV99] I. Kovacs, D. S. Silver, and S. G. Williams: Determinants of commuting-block matrices, Am. Math. Mon. 106, 950-952 (1999).

[KRI17] S. Krishnagopal, J. Lehnert, W. Poel, A. Zakharova, and E. Schöll: Synchronization patterns: From network motifs to hierarchical networks, Phil. Trans. R. Soc. A 375, 20160216 (2017).

[KUE15] C. Kuehn: Multiple Time Scale Dynamics (Springer, Cham, 2015).

[KUR84] Y. Kuramoto: Chemical Oscillations, Waves and Turbulence (Springer-Verlag, Berlin, 1984). 
[KUR02a] Y. Kuramoto and D. Battogtokh: Coexistence of Coherence and Incoherence in Nonlocally Coupled Phase Oscillators., Nonlin. Phen. in Complex Sys. 5, 380-385 (2002).

[KYR14] Y. N. Kyrychko, K. B. Blyuss, and E. Schöll: Synchronization of networks of oscillators with distributed-delay coupling, Chaos 24, 043117 (2014).

[LUE12a] L. Lücken and S. Yanchuk: Two-cluster bifurcations in systems of globally pulse-coupled oscillators, Physica D 241, 350-359 (2012).

[LUE16] L. Lücken, O. V. Popovych, P. Tass, and S. Yanchuk: Noise-enhanced coupling between two oscillators with long-term plasticity, Phys. Rev. E 93, 032210 (2016).

[LAD13] J. Ladenbauer, J. Lehnert, H. Rankoohi, T. Dahms, E. Schöll, and K. Obermayer: Adaptation controls synchrony and cluster states of coupled threshold-model neurons, Phys. Rev. E 88, 042713 (2013).

[LAI09] C. R. Laing: The dynamics of chimera states in heterogeneous Kuramoto networks, Physica D 238, 1569-1588 (2009).

[LAN05a] C. E. Landisman and B. W. Connors: Long-term modulation of electrical synapses in the mammalian thalamus, Science 310, 1809-1813 (2005).

[LAR13] L. Larger, B. Penkovsky, and Y. Maistrenko: Virtual chimera states for delayed-feedback systems, Phys. Rev. Lett. 111, 054103 (2013).

[LAR15] L. Larger, B. Penkovsky, and Y. Maistrenko: Laser chimeras as a paradigm for multistable patterns in complex systems, Nat. Commun. 6, 7752 (2015).

[LEH14] J. Lehnert, P. Hövel, A. A. Selivanov, A. L. Fradkov, and E. Schöll: Controlling cluster synchronization by adapting the topology, Phys. Rev. E 90, 042914 (2014).

[LEH15b] J. Lehnert: Controlling synchronization patterns in complex networks, Springer Theses (Springer, Heidelberg, 2016).

[LEO19] I. León and D. Pazó: Phase reduction beyond the first order: The case of the mean-field complex ginzburg-landau equation, Phys. Rev. E 100, 012211 (2019).

[LET07] J. J. Letzkus, B. M. Kampa, and G. J. Stuart: Does spike timing-dependent synaptic plasticity underlie memory formation?, Clin. Exp. Pharmacol. Physiol. 34, 1070-1076 (2007).

[LEY17a] I. Leyva, R. Sevilla-Escoboza, I. Sendiña-Nadal, R. Gutiérrez, J. M. Buldú, and S. Boccaletti: Inter-layer synchronization in non-identical multi-layer networks, Sci. Rep. 7, 45475 (2017).

[LEY18] I. Leyva, I. Sendiña-Nadal, R. Sevilla-Escoboza, V. P. Vera-Avila, P. Chholak, and S. Boccaletti: Relay synchronization in multiplex networks, Sci. Rep. 8, 8629 (2018).

[LI10c] M. Li, S. Guan, and C. H. Lai: Spontaneous formation of dynamical groups in an adaptive networked system, New J. Phys. 12, 103032 (2010).

[LI11a] M. H. Li, S. G. Guan, and C. H. Lai: Formation of modularity in a model of evolving networks, Europhys. Lett. 95, 58004 (2011). 
[LIE15] J. Liesen and V. Mehrmann: Linear Algebra (Springer, Cham, 2015).

[MAC08] B. D. MacArthur, R. J. Sanchez-Garcia, and J. W. Anderson: Symmetry in complex networks, Discrete Appl. Math. 156, 3525-3531 (2008).

[MAC09a] B. D. MacArthur and R. J. Sanchez-Garcia: Spectral characteristics of network redundancy, Phys. Rev. E 80, 026117 (2009).

[MAG12a] C. Magri, U. Schridde, Y. Murayama, and S. Panzeri: The amplitude and timing of the bold signal reflects the relationship between local field potential power at different frequencies, J. Neurosci. 32, 1395-1407 (2012).

[MAI07] Y. Maistrenko, B. Lysyansky, C. Hauptmann, O. Burylko, and P. A. Tass: Multistability in the kuramoto model with synaptic plasticity, Phys. Rev. E 75, 066207 (2007).

[MAI14a] Y. Maistrenko, B. Penkovsky, and M. Rosenblum: Solitary state at the edge of synchrony in ensembles with attractive and repulsive interactions, Phys. Rev. E 89, 060901 (2014).

[MAI14] Y. Maistrenko, A. Vasylenko, O. Sudakov, R. Levchenko, and V. L. Maistrenko: Cascades of multi-headed chimera states for coupled phase oscillators, Int. J. Bifur. Chaos 24, 1440014 (2014).

[MAI17] Y. Maistrenko, S. Brezetsky, P. Jaros, R. Levchenko, and T. Kapitaniak: Smallest chimera states, Phys. Rev. E 95, 010203(R) (2017).

[MAI18] D. M. N. Maia, E. E. N. Macau, T. Pereira, and S. Yanchuk: Synchronization in networks with strongly delayed couplings, Discr. Cont. Dyn. Syst. B 23, 3461-3482 (2018).

[MAJ18a] S. Majhi, B. K. Bera, D. Ghosh, and M. Perc: Chimera states in neuronal networks: A review, Phys. Life Rev. 26, 100-121 (2019).

[MAK16a] V. V. Makarov, A. A. Koronovskii, V. A. Maksimenko, A. E. Hramov, O. I. Moskalenko, J. M. Buldú, and S. Boccaletti: Emergence of a multilayer structure in adaptive networks of phase oscillators, Chaos Solitons Fractals 84, 23-30 (2016).

[MAK16] V. A. Maksimenko, V. V. Makarov, B. K. Bera, D. Ghosh, S. K. Dana, M. V. Goremyko, N. S. Frolov, A. A. Koronovskii, and A. E. Hramov: Excitation and suppression of chimera states by multiplexing, Phys. Rev. E 94, 052205 (2016).

[MAN07] D. Mantini, M. G. Perrucci, C. Del Gratta, G. L. Romani, and M. Corbetta: Electrophysiological signatures of resting state networks in the human brain, Proc. Natl. Acad. Sci. U.S.A. 104, 13170-13175 (2007).

[MAR97a] H. Markram, J. Lübke, M. Frotscher, and B. Sakmann: Regulation of synaptic efficacy by coincidence of postsynaptic APs and EPSPs., Science 275, 213-215 (1997).

[MAR09c] S. A. Marvel, R. E. Mirollo, and S. H. Strogatz: Identical phase oscillators with global sinusoidal coupling evolve by möbius group action, Chaos 19, 043104 (2009).

[MAR11b] H. Markram, W. Gerstner, and P. J. Sjöström: A history of spike-timing-dependent plasticity, Front. Synaptic Neurosci. 3 (2011).

[MAR12c] H. Markram: The human brain project, Scientific American 306, 50-55 (2012). 
[MAR13] E. A. Martens, S. Thutupalli, A. Fourriere, and O. Hallatschek: Chimera states in mechanical oscillator networks, Proc. Natl. Acad. Sci. USA 110, 10563 (2013).

[MAS07c] N. Masuda and H. Kori: Formation of feedforward networks and frequency synchrony by spike-timing-dependent plasticity, J. Comp. Neurosci. 22, 327-345 (2007).

[MAS17c] O. V. Maslennikov and V. I. Nekorkin: Adaptive dynamical networks, Phys. Usp. 60, 694-704 (2017).

[MAT14] A. Mathy, B. A. Clark, and M. Häusser: Synaptically induced long-term modulation of electrical coupling in the inferior olive, Neuron 81, 1290-1296 (2014).

[MEH17a] V. Mehrmann, R. Morandin, S. Olmi, and E. Schöll: Qualitative stability and synchronicity analysis of power network models in port-hamiltonian form, arXiv, 1712.03160 (2017).

[MEH18] V. Mehrmann, R. Morandin, S. Olmi, and E. Schöll: Qualitative stability and synchronicity analysis of power network models in port-Hamiltonian form, Chaos 28, 101102 (2018).

[MEI09a] C. Meisel and T. Gross: Adaptive self-organization in a realistic neural network model, Phys. Rev. E 80, 061917 (2009).

[MEN14] P. J. Menck, J. Heitzig, J. Kurths, and H. J. Schellnhuber: How dead ends undermine power grid stability, Nat. Commun. 5, 3969 (2014).

[MER14] E. Mercier, D. Wolfersberger, and M. Sciamanna: Bifurcation to chaotic low-frequency fluctuations in a laser diode with phase-conjugate feedback, Opt. Lett. 39, 4021-4024 (2014).

[MEU10a] D. Meunier, R. Lambiotte, and E. T. Bullmore: Modular and hierarchically modular organization of brain networks, Front. Neurosci. 4, 200 (2010).

[MIK14] K. Mikkelsen, A. Imparato, and A. Torcini: Sisyphus effect in pulse-coupled excitatory neural networks with spike-timing-dependent plasticity, Phys. Rev. E 89, 062701 (2014).

[MIK18] M. Mikhaylenko, L. Ramlow, S. Jalan, and A. Zakharova: Weak multiplexing in neural networks: Switching between chimera and solitary states, Chaos 29, 023122 (2019).

[MIL13b] A. Miller and D. Z. Jin: Potentiation decay of synapses and length distributions of synfire chains self-organized in recurrent neural networks, Phys. Rev. E 88, 062716 (2013).

[MON08] S. Monto, S. Palva, J. Voipio, and J. M. Palva: Very slow eeg fluctuations predict the dynamics of stimulus detection and oscillation amplitudes in humans, J. Neurosci. 28, 8268-8272 (2008).

[MOT10] A. E. Motter: Nonlinear dynamics: Spontaneous synchrony breaking, Nat. Phys. 6, 164-165 (2010).

[MOT13a] A. E. Motter, S. A. Myers, M. Anghel, and T. Nishikawa: Spontaneous synchrony in power-grid networks, Nat. Phys. 9, 191-197 (2013).

[NEK15b] V. I. Nekorkin: Introduction to Nonlinear Oscillations (Wiley, Weinheim, 2015).

[NEK16] V. I. Nekorkin and D. V. Kasatkin: Dynamics of a network of phase oscillators with plastic couplings, AIP Conf. Proc. 1738, 210010 (2016). 
[NEW03] M. E. J. Newman: The structure and function of complex networks, SIAM Review 45, 167-256 (2003).

[NEW10] M. E. J. Newman: Networks: an introduction (Oxford University Press, Inc., New York, 2010).

[NIC92] S. Nichols and K. Wiesenfeld: Ubiquitous neutral stability of splay-phase states, Phys. Rev. A 45, 8430-8435 (1992).

[NIC13] V. Nicosia, M. Valencia, M. Chavez, A. Díaz-Guilera, and V. Latora: Remote synchronization reveals network symmetries and functional modules, Phys. Rev. Lett. 110, 174102 (2013).

[NIK19] D. Nikitin, I. Omelchenko, A. Zakharova, M. Avetyan, A. L. Fradkov, and E. Schöll: Complex partial synchronization patterns in networks of delay-coupled neurons, Phil. Trans. R. Soc. A 377, 20180128 (2019).

[NIS06] T. Nishikawa and A. E. Motter: Synchronization is optimal in nondiagonalizable networks, Phys. Rev. E 73, 065106 (2006).

[NIY09] R. K. Niyogi and L. Q. English: Learning-rate-dependent clustering and self-development in a network of coupled phase oscillators, Phys. Rev. E 80, 066213 (2009).

[OLM15a] S. Olmi: Chimera states in coupled kuramoto oscillators with inertia, Chaos 25, 123125 (2015).

[OLM15] S. Olmi, E. A. Martens, S. Thutupalli, and A. Torcini: Intermittent chaotic chimeras for coupled rotators, Phys. Rev. E 92, 030901(R) (2015).

[OME08] O. E. Omel'chenko, Y. Maistrenko, and P. Tass: Chimera states: The natural link between coherence and incoherence, Phys. Rev. Lett. 100, 044105 (2008).

[OME11] I. Omelchenko, Y. Maistrenko, P. Hövel, and E. Schöll: Loss of coherence in dynamical networks: spatial chaos and chimera states, Phys. Rev. Lett. 106, 234102 (2011).

[OME12b] O. E. Omel'chenko and M. Wolfrum: Nonuniversal transitions to synchrony in the sakaguchi-kuramoto model, Phys. Rev. Lett. 109, 164101 (2012).

[OME13] I. Omelchenko, O. E. Omel'chenko, P. Hövel, and E. Schöll: When nonlocal coupling between oscillators becomes stronger: patched synchrony or multichimera states, Phys. Rev. Lett. 110, 224101 (2013).

[OME15] I. Omelchenko, A. Provata, J. Hizanidis, E. Schöll, and P. Hövel: Robustness of chimera states for coupled FitzHugh-Nagumo oscillators, Phys. Rev. E 91, 022917 (2015).

[OME16] I. Omelchenko, O. E. Omel'chenko, A. Zakharova, M. Wolfrum, and E. Schöll: Tweezers for chimeras in small networks, Phys. Rev. Lett. 116, 114101 (2016).

[OME18] I. Omelchenko, O. E. Omel'chenko, A. Zakharova, and E. Schöll: Optimal design of tweezer control for chimera states, Phys. Rev. E 97, 012216 (2018).

[OME18a] O. E. Omel'chenko: The mathematics behind chimera states, Nonlinearity 31, R121 (2018). 
[OME19] I. Omelchenko, T. Hülser, A. Zakharova, and E. Schöll: Control of chimera states in multilayer networks, Front. Appl. Math. Stat. 4, 67 (2019).

[OME19c] O. E. Omel'chenko and E. Knobloch: Chimerapedia: coherence-incoherence patterns in one, two and three dimensions, New J. Phys. 21, 093034 (2019).

[OTT08] E. Ott and T. M. Antonsen: Low dimensional behavior of large systems of globally coupled oscillators, Chaos 18, 037113 (2008).

[RAS06a] C. Pötzsche and M. Rasmussen: Taylor approximation of integral manifolds, J. Dyn. Diff. Equ. 18, 427-460 (2006).

[PON15] A. Ponce-Alvarez, G. Deco, P. Hagmann, G. L. Romani, D. Mantini, and M. Corbetta: Resting-state temporal synchronization networks emerge from connectivity topology and heterogeneity, PLoS Comp. Biol. 11, e1004100 (2015).

[PAI14] D. Pais and N. E. Leonard: Adaptive network dynamics and evolution of leadership in collective migration, Physica D 267, 81-93 (2014).

[PAN15] M. J. Panaggio and D. M. Abrams: Chimera states: Coexistence of coherence and incoherence in networks of coupled oscillators, Nonlinearity 28, R67 (2015).

[PAP17] L. Papadopoulos, J. Z. Kim, J. Kurths, and D. S. Bassett: Development of structural correlations and synchronization from adaptive rewiring in networks of kuramoto oscillators, Chaos 27, 073115 (2017).

[PAS95] F. Pasemann: Characterization of periodic attractors in neural ring networks, Neural Networks 8, 421-429 (1995).

[PAZ05a] D. Pazó: Thermodynamic limit of the first-order phase transition in the kuramoto model, Phys. Rev. E 72, 046211 (2005).

[PAZ05] D. Pazó, R. R. Deza, and V. Pérez-Muñuzuri: Parity-breaking front bifurcation in bistable media: link between discrete and continuous versions, Phys. Lett. A 340, 132-138 (2005).

[PEC98] L. M. Pecora and T. L. Carroll: Master Stability Functions for Synchronized Coupled Systems, Phys. Rev. Lett. 80, 2109-2112 (1998).

[PEC14] L. M. Pecora, F. Sorrentino, A. M. Hagerstrom, T. E. Murphy, and R. Roy: Symmetries, cluster synchronization, and isolated desynchronization in complex networks, Nat. Commun. 5, 4079 (2014).

[PEL20] K. Pelka, V. Peano, and A. Xuereb: Chimera states in small optomechanical arrays, Phys. Rev. Research 2, 013201 (2020).

[PEN19] B. Penkovsky, X. Porte, M. Jacquot, L. Larger, and D. Brunner: Coupled nonlinear delay systems as deep convolutional neural networks, Phys. Rev. Lett. 123, 054101 (2019).

[PER10c] P. Perlikowski, S. Yanchuk, O. V. Popovych, and P. Tass: Periodic patterns in a ring of delay-coupled oscillators, Phys. Rev. E 82, 036208 (2010).

[PER14a] A. E. Pereda: Electrical synapses and their functional interactions with chemical synapses, Nat. Rev. Neurosci. 15, 250-263 (2014). 
[PET12] S. Petkoski and A. Stefanovska: Kuramoto model with time-varying parameters, Phys. Rev. E 86, 046212 (2012).

[PIC11a] C. B. Picallo and H. Riecke: Adaptive oscillator networks with conserved overall coupling: Sequential firing and near-synchronized states, Phys. Rev. E 83, 036206 (2011).

[PIC13] M. D. Pickett, G. Medeiros-Ribeiro, and R. S. Williams: A scalable neuristor built with mott memristors, Nat. Mater. 12, 114-117 (2013).

[PIE19a] B. Pietras and A. Daffertshofer: Network dynamics of coupled oscillators and phase reduction techniques, Phys. Rep. 819, 1-105 (2019).

[PIK01] A. Pikovsky, M. Rosenblum, and J. Kurths: Synchronization: a universal concept in nonlinear sciences (Cambridge University Press, Cambridge, 1st edition, 2001).

[PIK08] A. Pikovsky and M. Rosenblum: Partially integrable dynamics of hierarchical populations of coupled oscillators, Phys. Rev. Lett. 101, 264103 (2008).

[PIK15] A. Pikovsky and M. Rosenblum: Dynamics of globally coupled oscillators: Progress and perspectives, Chaos 25, 097616 (2015).

[PIT18] E. Pitsik, V. Makarov, D. Kirsanov, N. Frolov, M. Goremyko, X. Li, Z. Wang, A. Hramov, and S. Boccaletti: Inter-layer competition in adaptive multiplex network, New J. Phys. 20, 075004 (2018).

[PLO16] S. A. Plotnikov, J. Lehnert, A. L. Fradkov, and E. Schöll: Adaptive control of synchronization in delay-coupled heterogeneous networks of FitzHugh-Nagumo nodes, Int. J. Bifurc. Chaos 26, 1650058 (2016).

[PLO16a] S. A. Plotnikov, J. Lehnert, A. L. Fradkov, and E. Schöll: Synchronization in heterogeneous FitzHugh-Nagumo networks with hierarchical architecture, Phys. Rev. E 94, 012203 (2016).

[POP11] O. V. Popovych, S. Yanchuk, and P. Tass: Delay- and coupling-induced firing patterns in oscillatory neural loops, Phys. Rev. Lett. 107, 228102 (2011).

[POP13] O. V. Popovych, S. Yanchuk, and P. Tass: Self-organized noise resistance of oscillatory neural networks with spike timing-dependent plasticity, Sci. Rep. 3, 2926 (2013).

[POP15] O. V. Popovych, M. N. Xenakis, and P. A. Tass: The spacing principle for unlearning abnormal neuronal synchrony, PLoS ONE 10, e0117205 (2015).

[POR16a] M. A. Porter and J. P. Gleeson: Dynamical Systems on Networks, vol. 4 of Frontiers in Applied Dynamical Systems: Reviews and Tutorials (Springer Int. Publ., 2016).

[POR19] M. A. Porter: Nonlinearity networks: A 2020 vision (2019), arxiv:1911.03805.

[POW11a] P. D. Powell: Calculating determinants of block matrices (2011), arXiv:1112.4379.

[PRE16] K. Premalatha, V. K. Chandrasekar, M. Senthilvelan, and M. Lakshmanan: Imperfectly synchronized states and chimera states in two interacting populations of nonlocally coupled Stuart-Landau oscillators, Phys. Rev. E 94, 012311 (2016). 
[RAM19] L. Ramlow, J. Sawicki, A. Zakharova, J. Hlinka, J. C. Claussen, and E. Schöll: Partial synchronization in empirical brain networks as a model for unihemispheric sleep, EPL 126, 50007 (2019), Highlighted in phys.org https://phys.org/news/2019-07unihemispheric-humans.html and in Europhys. News 50, no. 5-6 (2019).

[RAT00] N. C. Rattenborg, C. J. Amlaner, and S. L. Lima: Behavioral, neurophysiological and evolutionary perspectives on unihemispheric sleep, Neurosci. Biobehav. Rev. 24, 817-842 (2000).

[RAT16] N. C. Rattenborg, B. Voirin, S. M. Cruz, R. Tisdale, G. Dell'Omo, H. P. Lipp, M. Wikelski, and A. L. Vyssotski: Evidence that birds sleep in mid-flight, Nat. Commun. 7, 12468 (2016).

[REI17a] R. Reimbayev, K. Daley, and I. Belykh: When two wrongs make a right: synchronized neuronal bursting from combined electrical and inhibitory coupling., Phil. Trans. R. Soc. A 375, 20160282 (2017).

[REN07] Q. Ren and J. Zhao: Adaptive coupling and enhanced synchronization in coupled phase oscillators, Phys. Rev. E 76, 016207 (2007).

[REN14] Q. Ren, M. He, X. Yu, Q. Long, and J. Zhao: The adaptive coupling scheme and the heterogeneity in intrinsic frequency and degree distributions of the complex networks, Phys. Lett. A 378, 139-146 (2014).

[REQ16] R. J. Requejo and A. Díaz-Guilera: Replicator dynamics with diffusion on multiplex networks, Phys. Rev. E 94, 022301 (2016).

[RIE14] S. Rieubland, A. Roth, and M. Häusser: Structured connectivity in cerebellar inhibitory networks, Neuron 81, 913-929 (2014).

[RIN90] J. Rinzel: Mechanisms for nonuniform propagation along excitable cables, Ann. N. Y. Acad. Sci. 591, 51-61 (1990).

[ROD16] F. A. Rodrigues, T. K. D. M. Peron, P. Ji, and J. Kurths: The Kuramoto model in complex networks, Phys. Rep. 610, 1-98 (2016).

[ROH12] M. Rohden, A. Sorge, M. Timme, and D. Witthaut: Self-organized synchronization in decentralized power grids, Phys. Rev. Lett. 109, 064101 (2012).

[ROE19a] V. Röhr, R. Berner, E. L. Lameu, O. V. Popovych, and S. Yanchuk: Frequency cluster formation and slow oscillations in neural populations with plasticity, PLoS ONE 14, e0225094 (2019).

[ROS14a] D. P. Rosin, D. Rontani, N. Haynes, E. Schöll, and D. J. Gauthier: Transient scaling and resurgence of chimera states in coupled Boolean phase oscillators, Phys. Rev. E 90, 030902(R) (2014).

[ROS19b] M. Rosenblum and A. Pikovsky: Nonlinear phase coupling functions: a numerical study, Philos. Trans. Royal Soc. A 377, 20190093 (2019).

[ROS19a] M. Rosenblum and A. Pikovsky: Numerical phase reduction beyond the first order approximation, Chaos 29, 011105 (2019). 
[ROT14] A. Rothkegel and K. Lehnertz: Irregular macroscopic dynamics due to chimera states in small-world networks of pulse-coupled oscillators, New J. Phys. 16, 055006 (2014).

[RUB01] J. Rubin, D. D. Lee, and H. Sompolinsky: Equilibrium properties of temporally asymmetric hebbian plasticity, Phys. Rev. Lett. 86, 364 (2001).

[RUB09] M. Rubinov, O. Sporns, C. Van Leeuwen, and M. Breakspear: Symbiotic relationship between brain structure and dynamics, BMC Neuroscience 10, 55 (2009).

[RUZ19] G. Ruzzene, I. Omelchenko, E. Schöll, A. Zakharova, and R. G. Andrzejak: Controlling chimera states via minimal coupling modification, Chaos 29, 051103 (2019).

[RYB17] E. Rybalova, N. Semenova, G. Strelkova, and V. Anishchenko: Transition from complete synchronization to spatio-temporal chaos in coupled chaotic systems with nonhyperbolic and hyperbolic attractors, Eur. Phys. J. Spec. Top. 226, 1857 (2017).

[RYB19a] E. Rybalova, V. S. Anishchenko, G. I. Strelkova, and A. Zakharova: Solitary states and solitary state chimera in neural networks, Chaos 29, 071106 (2019).

[RYB19] E. Rybalova, T. Vadivasova, G. Strelkova, V. Anishchenko, and A. Zakharova: Forced synchronization of a multilayer heterogeneous network of chaotic maps in the chimera state mode, Chaos 29, 033134 (2019).

[SAK86] H. Sakaguchi and Y. Kuramoto: A soluble active rotater model showing phase transitions via mutual entertainment, Prog. Theor. Phys 76, 576-581 (1986).

[SAT19] K. Sathiyadevi, V. K. Chandrasekar, D. V. Senthilkumar, and M. Lakshmanan: Longrange interaction induced collective dynamical behaviors, J. Phys. A: Math. Theor. 52, 184001 (2019).

[SAW18c] J. Sawicki, I. Omelchenko, A. Zakharova, and E. Schöll: Delay controls chimera relay synchronization in multiplex networks, Phys. Rev. E 98, 062224 (2018).

[SAW18] J. Sawicki, I. Omelchenko, A. Zakharova, and E. Schöll: Synchronization scenarios of chimeras in multiplex networks, Eur. Phys. J. Spec. Top. 227, 1161 (2018).

[SAW20] J. Sawicki: Delay controlled partial synchronization in complex networks, Springer Theses (Springer, Heidelberg, 2019).

[SAW19] J. Sawicki, I. Omelchenko, A. Zakharova, and E. Schöll: Delay-induced chimeras in neural networks with fractal topology, Eur. Phys. J. B 92, 54 (2019).

[SAY13] H. Sayama, I. Pestov, J. Schmidt, B. J. Bush, C. Wong, J. Yamanoi, and T. Gross: Modeling complex systems with adaptive networks, Comput. Math. Appl. 65, 1645-1664 (2013).

[SAY15] H. Sayama and R. Sinatra: Social diffusion and global drift on networks, Phys. Rev. E 91, 032809 (2015).

[SCA15] F. Scafuti, T. Aoki, and M. di Bernardo: Heterogeneity induces emergent functional networks for synchronization, Phys. Rev. E 91, 062913 (2015).

[SCH87] E. Schöll: Nonequilibrium Phase Transitions in Semiconductors (Springer, Berlin, 1987). 
[SCH01] E. Schöll: Nonlinear spatio-temporal dynamics and chaos in semiconductors (Cambridge University Press, Cambridge, 2001), Nonlinear Science Series, Vol. 10.

[SCH07] E. Schöll and H. G. Schuster (Editors): Handbook of Chaos Control (Wiley-VCH, Weinheim, 2008), Second completely revised and enlarged edition.

[SCH12] E. Schöll, A. A. Selivanov, J. Lehnert, T. Dahms, P. Hövel, and A. L. Fradkov: Control of synchronization in delay-coupled networks, Int. J. Mod. Phys. B 26, 1246007 (2012).

[SCH14a] L. Schmidt, K. Schönleber, K. Krischer, and V. García-Morales: Coexistence of synchrony and incoherence in oscillatory media under nonlinear global coupling, Chaos 24, 013102 (2014).

[SCH14m] K. Schmietendorf, J. Peinke, R. Friedrich, and O. Kamps: Self-organized synchronization and voltage stability in networks of synchronous machines, Eur. Phys. J. Spec. Top. 223, 2577 (2014).

[SCH16a] E. Schöll: Chimera states and excitation waves in networks with complex topologies, AIP Conf. Proc. 1738, 210012 (2016).

[SCH16b] E. Schöll: Synchronization patterns and chimera states in complex networks: interplay of topology and dynamics, Eur. Phys. J. Spec. Top. 225, 891-919 (2016).

[SCH16] E. Schöll, S. H. L. Klapp, and P. Hövel: Control of self-organizing nonlinear systems (Springer, Berlin, 2016).

[SCH17m] M. Schröder, M. Timme, and D. Witthaut: A universal order parameter for synchrony in networks of limit cycle oscillators, Chaos 27, 073119 (2017).

[SCH17k] C. D. Schuman, T. E. Potok, R. M. Patton, J. D. Birdwell, M. E. Dean, G. S. Rose, and J. S. Plank: A survey of neuromorphic computing and neural networks in hardware (2017), arXiv:1705.06963.

[SCH18i] B. Schäfer, D. Witthaut, M. Timme, and V. Latora: Dynamically induced cascading failures in power grids, Nat. Commun. 9, 1975 (2018).

[SCH19c] E. Schöll, A. Zakharova, and R. G. Andrzejak: Editorial on the research topic: Chimera states in complex networks, Front. Appl. Math. Stat. 5, 62 (2019), doi: 10.3389/fams.2019.00062.

[SCH19a] L. Schülen, S. Ghosh, A. D. Kachhvah, A. Zakharova, and S. Jalan: Delay engineered solitary states in complex networks, Chaos Solitons Fractals 128, 290 (2019).

[SEV16] R. Sevilla-Escoboza, I. Sendiña-Nadal, I. Leyva, R. Gutiérrez, J. M. Buldú, and S. Boccaletti: Inter-layer synchronization in multiplex networks of identical layers, Chaos 26, 065304 (2016).

[SEL02] P. Seliger, S. C. Young, and L. S. Tsimring: Plasticity and learning in a network of coupled phase oscillators, Phys. Rev. E 65, 041906 (2002).

[SEL12] A. A. Selivanov, J. Lehnert, T. Dahms, P. Hövel, A. L. Fradkov, and E. Schöll: Adaptive synchronization in delay-coupled networks of Stuart-Landau oscillators, Phys. Rev. E 85, 016201 (2012). 
[SEM15b] V. Semenov, A. Zakharova, Y. Maistrenko, and E. Schöll: Delayed-feedback chimera states: Forced multiclusters and stochastic resonance, Europhys. Lett. 115, 10005 (2016).

[SEM18a] N. Semenova, T. Vadivasova, and V. Anishchenko: Mechanism of solitary state appearance in an ensemble of nonlocally coupled Lozi maps, Eur. Phys. J. Spec. Top. 227, 1173 (2018).

[SEM18] N. Semenova and A. Zakharova: Weak multiplexing induces coherence resonance, Chaos 28, 051104 (2018).

[SHA92] M. P. Shaw, V. V. Mitin, E. Schöll, and H. L. Grubin: The Physics of Instabilities in Solid State Electron Devices (Plenum Press, New York, 1992).

[SHA13b] S. Shai and S. Dobson: Coupled adaptive complex networks, Phys. Rev. E 87, 042812 (2013).

[SHU13] D. E. Shulz and D. E. Feldman: Chapter 9 - Spike Timing-Dependent Plasticity, in Neural Circuit Development and Function in the Brain, edited by (Academic Press, 2013), pp. 155-181.

[SIL00] J. R. Silvester: Determinants of block matrices, Math. Gaz. 84, 460-467 (2000).

[SIN99] W. Singer: Neuronal Synchrony: A Versatile Code Review for the Definition of Relations?, Neuron 24, 49-65 (1999).

[SIZ19] A. E. Sizemore, J. E. Phillips-Cremins, R. Ghrist, and D. S. Bassett: The importance of the whole: Topological data analysis for the network neuroscientist, Netw. Neurosci. 3, 656-673 (2019).

[SJO08] P. J. Sjöström, E. A. Rancz, A. Roth, and M. Häusser: Dendritic excitability and synaptic plasticity, Physiol. Rev. 88, 769-840 (2008).

[SKA13a] P. S. Skardal, D. Taylor, and J. G. Restrepo: Complex macroscopic behavior in systems of phase oscillators with adaptive coupling, Physica D 267, 27-35 (2013).

[SKA19] P. S. Skardal and A. Arenas: Abrupt desynchronization and extensive multistability in globally coupled oscillator simplexes, Phys. Rev. Lett. 122, 248301 (2019).

[SMI19] L. D. Smith and G. A. Gottwald: Chaos in networks of coupled oscillators with multimodal natural frequency distributions, Chaos 29, 093127 (2019).

[SMI20] L. D. Smith and G. A. Gottwald: Model reduction for the collective dynamics of globally coupled oscillators: A comparison between the collective coordinate framework and the ottantonsen ansatz (2019), arXiv:1910.00732.

[SON00] S. Song, K. D. Miller, and L. F. Abbott: Competitive hebbian learning through spike-timingdependent synaptic plasticity, Nat. Neurosci. 3, 919-926 (2000).

[SOR07] F. Sorrentino and E. Ott: Network synchronization of groups, Phys. Rev. E 76, 056114 (2007).

[SOR08] F. Sorrentino and E. Ott: Adaptive synchronization of dynamics on evolving complex networks, Phys. Rev. Lett. 100, 114101 (2008). 
[SOR16a] F. Sorrentino, L. M. Pecora, A. M. Hagerstrom, T. E. Murphy, and R. Roy: Complete characterization of the stability of cluster synchronization in complex dynamical networks, Sci. Adv. 2, e1501737 (2016).

[SOT17] N. Sothanaphan: Determinants of block matrices with noncommuting blocks, Lin. Alg. Applic. 512, 202-218 (2017).

[SPO11] O. Sporns: Networks of the brain (MIT Press, Cambridge, MA, USA, 2011).

[SOL13a] A. Solé-Ribalta, M. De Domenico, N. E. Kouvaris, A. Díaz-Guilera, S. Gómez, and A. Arenas: Spectral properties of the laplacian of multiplex networks, Phys. Rev. E 88, 032807 (2013).

[STA10b] C. J. Stam, A. Hillebrand, H. Wang, and P. Van Mieghem: Emergence of modular structure in a large-scale brain network with interactions between dynamics and connectivity, Front. Comput. Neurosci. 4, 133 (2010).

[STE11b] I. Stewart: Phase oscillators with sinusoidal coupling interpreted in terms of projective geometry, Int. J. Bifurc. Chaos 21, 1795-1804 (2011).

[STR93a] S. H. Strogatz and R. E. Mirollo: Splay states in globally coupled Josephson arrays: Analytical prediction of Floquet multipliers, Phys. Rev. E 47, 220-227 (1993).

[STR93] S. H. Strogatz and I. Stewart: Coupled oscillators and biological synchronization, Sci. Am. 269, 102-109 (1993).

[STR00] S. H. Strogatz: From Kuramoto to Crawford: exploring the onset of synchronization in populations of coupled oscillators, Physica D 143, 1-20 (2000).

[STR01a] S. H. Strogatz: Exploring complex networks, Nature 410, 268-276 (2001).

[STR03] S. H. Strogatz: Sync: How order emerges from chaos in the universe, nature, and daily life (Hyperion, New York, 2003).

[STR05a] S. H. Strogatz, D. Abraham, A. D. McRobbie, B. Eckhardt, and E. Ott: Crowd synchrony on the millennium bridge, Nature 438, 43-44 (2005).

[TAH19] H. Taher, S. Olmi, and E. Schöll: Enhancing power grid synchronization and stability through time delayed feedback control, Phys. Rev. E 100, 062306 (2019).

[TAK09] Y. K. Takahashi, H. Kori, and N. Masuda: Self-organization of feed-forward structure and entrainment in excitatory neural networks with spike-timing-dependent plasticity, Phys. Rev. E 79, 051904 (2009).

[TAN11b] T. Tanaka and T. Aoyagi: Multistable attractors in a network of phase oscillators with three-body interactions, Phys. Rev. Lett. 106, 224101 (2011).

[TAN19] L. Tang, X. Wu, J. Lü, J. Lu, and R. M. D'Souza: Master stability functions for complete, intralayer, and interlayer synchronization in multiplex networks of coupled rössler oscillators, Phys. Rev. E 99 (2019), 012304.

[TAS06] P. A. Tass and M. Majtanik: Long-term anti-kindling effects of desynchronizing brain stimulation: a theoretical study, Biol. Cybern. 94, 58-66 (2006). 
[TAS12] P. A. Tass, I. Adamchic, H. J. Freund, T. von Stackelberg, and C. Hauptmann: Counteracting tinnitus by acoustic coordinated reset neuromodulation, Restor. Neurol. Neurosci. 30, 137-159 (2012).

[TAS12a] P. A. Tass and O. V. Popovych: Unlearning tinnitus-related cerebral synchrony with acoustic coordinated reset stimulation: theoretical concept and modelling, Biol. Cybern. 106, 27-36 (2012).

[TAY10] D. Taylor, E. Ott, and J. G. Restrepo: Spontaneous synchronization of coupled oscillator systems with frequency adaptation, Phys. Rev. E 81, 046214 (2010).

[TSI16] N. D. Tsigkri-DeSmedt, J. Hizanidis, P. Hövel, and A. Provata: Multi-chimera states and transitions in the leaky integrate-and-fire model with excitatory coupling and hierarchical connectivity, Eur. Phys. J. ST 225, 1149 (2016).

[TEI19] E. Teichmann and M. Rosenblum: Solitary states and partial synchrony in oscillatory ensembles with attractive and repulsive interactions, Chaos 29, 093124 (2019).

[TIM14] L. Timms and L. Q. English: Synchronization in phase-coupled Kuramoto oscillator networks with axonal delay and synaptic plasticity, Phys. Rev. E 89, 032906 (2014).

[TIN12] M. R. Tinsley, S. Nkomo, and K. Showalter: Chimera and phase cluster states in populations of coupled chemical oscillators, Nat. Phys. 8, 662-665 (2012).

[TOT15] J. Totz, R. Snari, D. Yengi, M. R. Tinsley, H. Engel, and K. Showalter: Phase-lag synchronization in networks of coupled chemical oscillators, Phys. Rev. E 92, 022819 (2015).

[TOT18] J. Totz, J. Rode, M. R. Tinsley, K. Showalter, and H. Engel: Spiral wave chimera states in large populations of coupled chemical oscillators, Nat. Phys. 14, 282-285 (2018).

[TOT20] C. H. Totz, S. Olmi, and E. Schöll: Control of synchronization in two-layer power grids, Phys. Rev. E 102, 022311 (2020).

[TRU13] P. A. Truitt, J. B. Hertzberg, E. Altunkaya, and K. C. Schwab: Linear and nonlinear coupling between transverse modes of a nanomechanical resonator, J. Appl. Phys. 114, 114307 (2013).

[TUM18] L. Tumash, S. Olmi, and E. Schöll: Effect of disorder and noise in shaping the dynamics of power grids, Europhys. Lett. 123, 20001 (2018).

[TUM19a] L. Tumash, A. Zakharova, E. Panteley, and E. Schöll: Synchronization patterns in Stuart-Landau networks: a reduced system approach, Eur. Phys. J. B 92, 100 (2019).

[UHL06] P. J. Uhlhaas and W. Singer: Neural synchrony in brain disorders: Relevance for cognitive dysfunctions and pathophysiology, Neuron 52, 155-168 (2006).

[ULO16] S. Ulonska, I. Omelchenko, A. Zakharova, and E. Schöll: Chimera states in networks of Van der Pol oscillators with hierarchical connectivities, Chaos 26, 094825 (2016).

[VAI18] M. Vaiana and S. F. Muldoon: Multilayer brain networks, J. Nonlinear Sci., 1-23 (2018).

[VER06] F. Verhulst: Methods and applications of singular perturbations: boundary layers and multiple timescale dynamics (Springer, 2006). 
[WAL13] M. M. Waldrop: Neuroelectronics: Smart connections, Nature 503, 22-24 (2013).

[WAN92] X. J. Wang and J. Rinzel: Alternating and synchronous rhythms in reciprocally inhibitory model neurons, Neural comp 4, 84-97 (1992).

[WAN08f] L. Wang, H. P. Dai, H. Dong, Y. Y. Cao, and Y. X. Sun: Adaptive synchronization of weighted complex dynamical networks through pinning, Eur. Phys. J. B 61, 335-342 (2008).

[WAR14] L. Wardil and C. Hauert: Origin and structure of dynamic cooperative networks, Sci. Rep. 4 (2014).

[WAT93a] S. Watanabe and S. H. Strogatz: Integrability of a globally coupled oscillator array, Phys. Rev. Lett. 70, 2391 (1993).

[WAT94] S. Watanabe and S. H. Strogatz: Constants of motion for superconducting Josephson arrays, Physica D 74, 197-253 (1994).

[WAT98a] D. J. Watts and S. H. Strogatz: Collective dynamics of 'small-world' networks, Nature 393, 440 (1998).

[WHI98] J. A. White, C. C. Chow, J. Ritt, C. Soto-Trevino, and N. Kopell: Synchronization and oscillatory dynamics in heterogeneous, mutually inhibited neurons, J. Comp. Neurosci. 5, 5-16 (1998).

[WIC13] M. Wickramasinghe and I. Z. Kiss: Spatially organized dynamical states in chemical oscillator networks: Synchronization, dynamical differentiation, and chimera patterns, PLoS ONE 8, e80586 (2013).

[WIC14] M. Wickramasinghe and I. Z. Kiss: Spatially organized partial synchronization through the chimera mechanism in a network of electrochemical reactions, Phys. Chem. Chem. Phys. 16, 18360-18369 (2014).

[WIL06] D. A. Wiley, S. H. Strogatz, and M. Girvan: The size of the sync basin, Chaos 16, 015103 (2006).

[WIL12] M. Wildie and M. Shanahan: Hierarchical clustering identifies hub nodes in a model of resting-state brain activity, in Neural Networks (IJCNN), The 2012 International Joint Conference on, edited by (IEEE, 2012), pp. 1-6.

[WIL14] C. Wille, J. Lehnert, and E. Schöll: Synchronization-desynchronization transitions in complex networks: An interplay of distributed time delay and inhibitory nodes, Phys. Rev. E 90, 032908 (2014).

[WIN80] A. T. Winfree: The Geometry of Biological Time (Springer, New York, 1980).

[WIN01] A. T. Winfree: The Geometry of Biological Time (Springer-Verlag New York, 2001).

[WIN12] M. Winkler, S. Butscher, and W. Kinzel: Pulsed chaos synchronization in networks with adaptive couplings, Phys. Rev. E 86, 016203 (2012).

[WIT06] G. M. Wittenberg and S. S. Wang: Malleability of spike-timing-dependent plasticity at the ca3-ca1 synapse, J. Neurosci. 26, 6610-6617 (2006). 
[WIT16] D. Witthaut, M. Rohden, X. Zhang, S. Hallerberg, and M. Timme: Critical links and nonlocal rerouting in complex supply networks, Phys. Rev. Lett. 116, 138701 (2016).

[WOJ16] J. Wojewoda, K. Czolczynski, Y. Maistrenko, and T. Kapitaniak: The smallest chimera state for coupled pendula, Sci. Rep. 6, 34329 (2016).

[WAN19b] R. Wong, P. Lin, M. Liu, Y. Wu, T. Zhou, and C. Zhou: Hierarchical connectome modes and critical state jointly maximize human brain functional diversity, Phys. Rev. Lett. 123, 038301 (2019).

[WU18a] H. Wu and M. Dhamala: Dynamics of kuramoto oscillators with time-delayed positive and negative couplings, Phys. Rev. E 98, 032221 (2018).

[XIA08] Y. Xiao, B. D. MacArthur, H. Wang, M. Xiong, and W. Wang: Network quotients: Structural skeletons of complex systems, Phys. Rev. E 78, 046102 (2008).

[YAN01a] S. Yanchuk, Y. Maistrenko, and E. Mosekilde: Partial synchronization and clustering in a system of diffusively coupled chaotic oscillators, Math. Comp. Simul. 54, 491-508 (2001).

[YAN08a] S. Yanchuk and M. Wolfrum: Destabilization patterns in chains of coupled oscillators, Phys. Rev. E 77, 26212 (2008).

[YAN11] S. Yanchuk, P. Perlikowski, O. V. Popovych, and P. Tass: Variability of spatio-temporal patterns in non-homogeneous rings of spiking neurons, Chaos 21, 047511 (2011).

[YAN15a] S. Yanchuk, P. Perlikowski, M. Wolfrum, A. Stefanski, and T. Kapitaniak: Amplitude equations for collective spatio-temporal dynamics in arrays of coupled systems, Chaos 25, 033113 (2015).

[YAO16] N. Yao and Z. Zheng: Chimera states in spatiotemporal systems: Theory and applications, Int. J. Mod. Phy. B 30, 1630002 (2016).

[YEU99a] M. K. S. Yeung and S. H. Strogatz: Time delay in the kuramoto model of coupled oscillators, Phys. Rev. Lett. 82, 648-651 (1999).

[YU12] W. Yu, P. DeLellis, G. Chen, M. di Bernardo, and J. Kurths: Distributed adaptive control of synchronization in complex networks, IEEE Trans. Autom. Control 57, 2153-2158 (2012).

[YUA11] W. J. Yuan and C. Zhou: Interplay between structure and dynamics in adaptive complex networks: Emergence and amplification of modularity by adaptive dynamics, Phys. Rev. E 84, 016116 (2011).

[ZAK14] A. Zakharova, M. Kapeller, and E. Schöll: Chimera death: Symmetry breaking in dynamical networks, Phys. Rev. Lett. 112, 154101 (2014).

[ZAK16b] M. A. Zaks and P. Tomov: Onset of time dependence in ensembles of excitable elements with global repulsive coupling, Phys. Rev. E 93, 020201 (2016).

[ZAK20] A. Zakharova: Chimera Patterns in Networks: Interplay between Dynamics, Structure, Noise, and Delay, Understanding Complex Systems (Springer, 2020).

[ZHA15a] X. Zhang, S. Boccaletti, S. Guan, and Z. Liu: Explosive synchronization in adaptive and multilayer networks, Phys. Rev. Lett. 114, 038701 (2015). 
[ZHA20] Y. Zhang and A. E. Motter: Symmetry-independent stability analysis of synchronization patterns, arXiv:2003.05461 (2020).

[ZHO06f] C. Zhou and J. Kurths: Dynamical weights and enhanced synchronization in adaptive complex networks, Phys. Rev. Lett. 96, 164102 (2006).

[ZHO06c] C. Zhou, L. Zemanová, G. Zamora, C. C. Hilgetag, and J. Kurths: Hierarchical organization unveiled by functional connectivity in complex brain networks, Phys. Rev. Lett. 97, 238103 (2006).

[ZHO07] C. Zhou, L. Zemanová, G. Zamora-López, C. C. Hilgetag, and J. Kurths: Structurefunction relationship in complex brain networks expressed by hierarchical synchronization, New J. Phys. 9, 178 (2007).

[ZHU10] J. F. Zhu, M. Zhao, W. Yu, C. Zhou, and B. H. Wang: Better synchronizability in generalized adaptive networks, Phys. Rev. E 81, 026201 (2010).

[ZOU09b] W. Zou and M. Zhan: Splay states in a ring of coupled oscillators: From local to global coupling, SIAM J. Appl. Dyn. Syst. 8, 1324-1340 (2009). 